\title{
Iron-Catalyzed Highly para-Selective Difluoromethylation of Arenes
}

Wei-Tai Fan, [a] Yuting Li, [a] Dongjie Wang, ${ }^{[a]}$ Shun-Jun Ji, ${ }^{*[a]}$ and Yingsheng Zhao*[a],[b]

\section{Table of Contents}

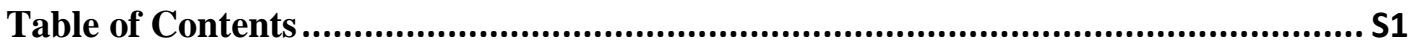

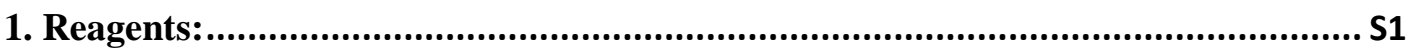

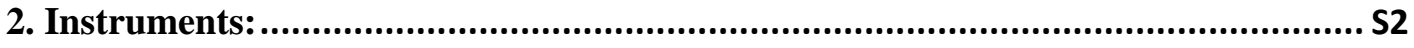

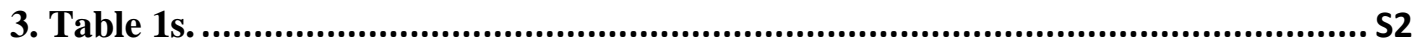

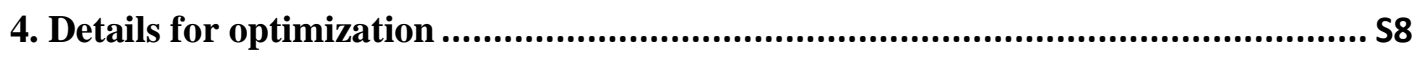

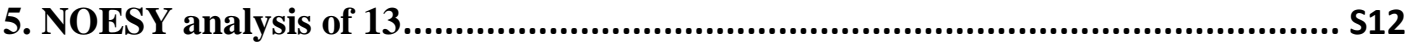

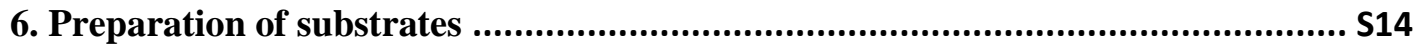

7. Procedures for preparation 3 ......................................................................... S18

8. Procedures for preparation 5 or 13....................................................................... S22

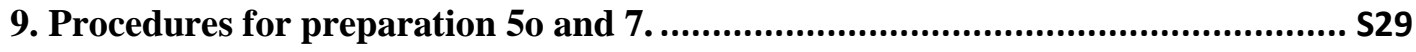

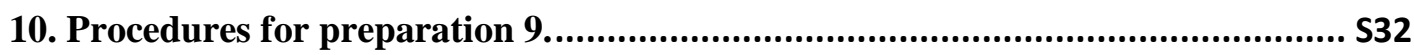

11. Procedures for preparation 5q............................................................................ S34

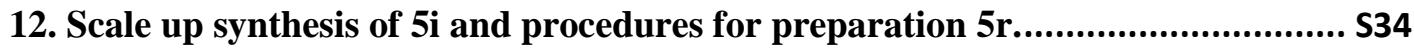

13. General Procedures for TEMPO Inhibition Experiments................................. S36

14. Radical trapping experiments....................................................................... S36

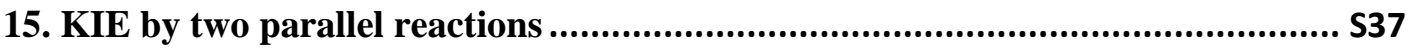

16. KIE by intermolecular competion ............................................................... S37

17. The characterization of $\mathrm{Fe}^{2+}$ by XPS .............................................................. S38

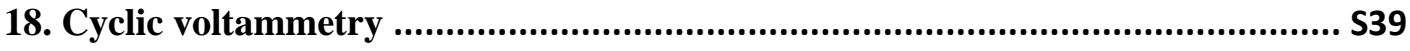

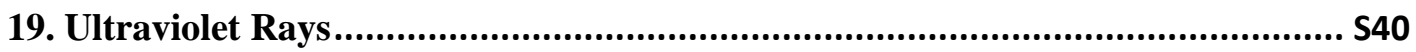

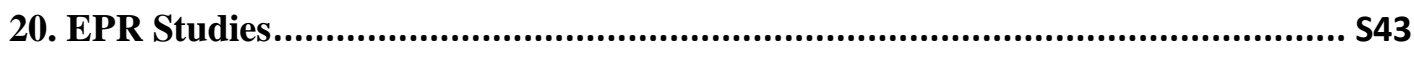

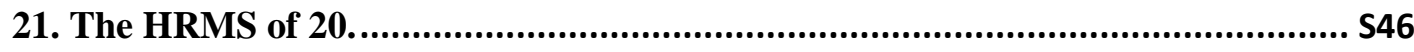

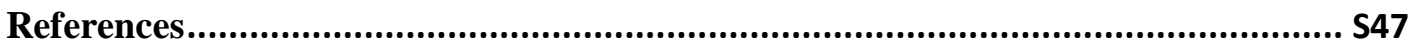

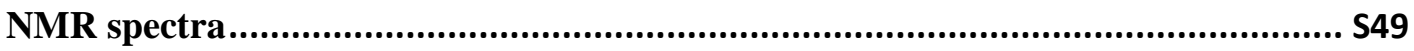

1. Reagents: Unless otherwise noted, all reagents were purchased from commercial suppliers 
and used without further purification. Column chromatography purifications were performed using 200-300 mesh silica gel.

2. Instruments: NMR spectra were recorded on Varian Inova- $400 \mathrm{MHz}$, Inova-300 MHz, Bruker DRX-400 or Bruker DRX-500 instruments and calibrated using residual solvent peaks as internal reference. Multiplicities are recorded as: $\mathrm{s}=\operatorname{singlet}, \mathrm{d}=$ doublet, $\mathrm{t}=$ triplet, $\mathrm{q}=$ quartet, $\mathrm{dd}=$ doublet of doublets, $\mathrm{m}=$ multiplet. HRMS analysis were carried out using a Bruker micrOTOF-Q instrument or a TOF-MS instrument. The XPS test was operated on X-ray photoelectron spectrometer (EXCALAB $250 \mathrm{XI}$ ). Cyclic voltammetry measurements were performed on CHI660D Electrochemical Workstation at $50 \mathrm{mVs}^{-1}$. The UV-vis diffuse reflection spectroscopy (DRS) were measured on a Shimadzu UV-3600 spectrophotometer at room temperature. Electron spin resonance (EPR) was utilizedto observe the signal peak of oxygen vacancies, N-t-Butyl-alpha-phenylnitrone( $\mathrm{PBN})$ and tert-Nitrosobutanedimer were used to detect free radicals on the JES-X320.

3. Table 1s. Optimization of reaction conditions. ${ }^{[a]}$

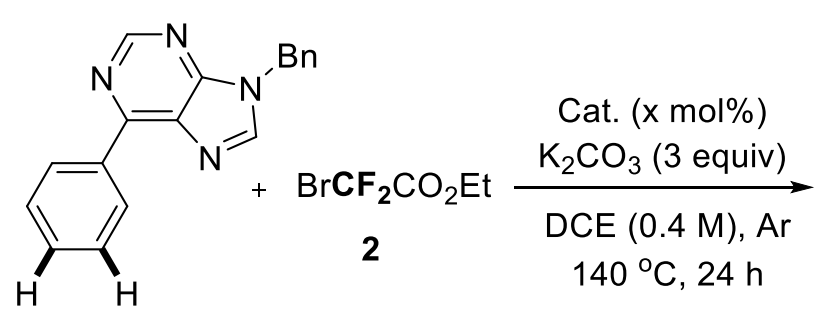

1a<smiles>CCOC(C)(F)c1ccc(C(F)(F)c2cccc(Cn3cnc4c(-c5ccc(C(F)(F)OCC)cc5)ncnc43)c2)cc1</smiles>

$3 a$
Ligand $\quad\left(\mathbf{3 a}+\mathbf{3} \mathbf{a}^{\prime}\right) \quad\left(\mathbf{3 a} / \mathbf{3} \mathbf{a}^{\prime}\right)$

Yield $(\%)^{b} \quad(\mathrm{p} / \mathrm{m})^{c}$

\begin{tabular}{|c|c|c|c|c|}
\hline entry & $(\mathrm{x} \mathrm{mol} \%)$ & & Yield $(\%)^{b}$ & $(\mathrm{p} / \mathrm{m})^{c}$ \\
\hline 1 & {$\left[\mathrm{RuCl}_{2}(\mathrm{p} \text {-cymene })\right]_{2}(5)$} & L1 & 51 & $2.7: 1$ \\
\hline $2^{d}$ & {$\left[\mathrm{RuCl}_{2}(\mathrm{p} \text {-cymene })\right]_{2}(5)$} & PivOH & 22 & $1: 1.5$ \\
\hline $3^{d}$ & {$\left[\mathrm{RuCl}_{2}(\mathrm{p} \text {-cymene })\right]_{2}(5)$} & $\mathbf{L 3}$ & 38 & $1: 1.5$ \\
\hline 4 & {$\left[\mathrm{RuCl}_{2}(\mathrm{p} \text {-cymene })\right]_{2}(5)$} & L4 & 42 & $1: 3.9$ \\
\hline 5 & {$\left[\mathrm{RuCl}_{2}(\mathrm{p} \text {-cymene })\right]_{2}(5)$} & L5 & trace & -- \\
\hline 6 & Hemin (5) & -- & 41 & $25: 1$ \\
\hline 7 & $\mathrm{Fe}(\mathrm{TPP}) \mathrm{Cl}(2.5)$ & -- & 78 & $20: 1$ \\
\hline 8 & CoTPP (2.5) & -- & trace & -- \\
\hline 9 & NiTPP (2.5) & -- & trace & -- \\
\hline 10 & СuTPP (2.5) & -- & trace & -- \\
\hline 11 & $\mathrm{Mn}(\mathrm{TPP}) \mathrm{Cl}(2.5)$ & -- & 50 & $17: 1$ \\
\hline
\end{tabular}


trace

14

$\mathrm{nr}$

${ }^{a}$ Reactions were performed with 1a $(0.2 \mathrm{mmol}), 2$ (1.2 mmol, $160 \mathrm{ul}, 6$ equiv ), Cat. (x mol\%), and $\mathrm{K}_{2} \mathrm{CO}_{3}$ (3 equiv) in DCE $(0.5 \mathrm{~mL}), \mathrm{Ar}, 140{ }^{\circ} \mathrm{C}, 24 \mathrm{~h} .{ }^{b}$ Yields of product after silica gel chromatography. ${ }^{c}$ The ratio of selectivity determined by ${ }^{1} \mathrm{H}$ NMR spectroscopy. ${ }^{d} \mathrm{~K}_{2} \mathrm{CO}_{3}(0.4 \mathrm{mmol}$, 2 equiv) was added at $120^{\circ} \mathrm{C}$. 
The ${ }^{1} \mathrm{H}$ NMR spectra of Entry 1, 2, 3, 4, 6, 7 and 11

\section{Entry 1}
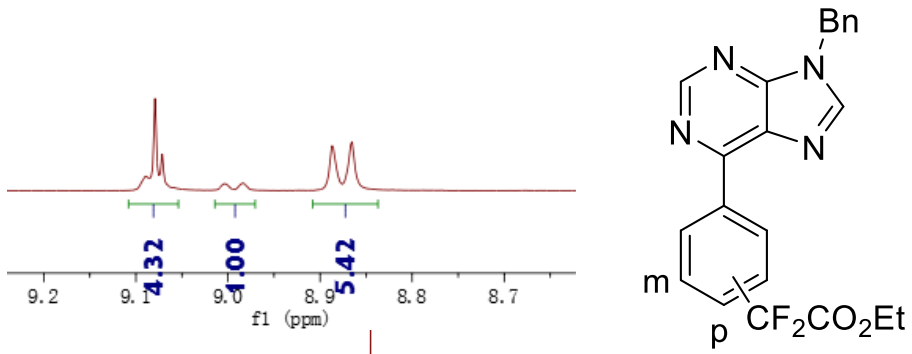

$\left(3 a+3 a^{\prime}\right), 51 \%$

$\mathrm{p} / \mathrm{m}=2.7: 1$

$\left[\mathrm{RuCl}_{2}(\mathrm{p} \text {-cymene })\right]_{2}(5 \mathrm{~mol} \%)$

L1 (10 mol\%)

$\mathrm{K}_{2} \mathrm{CO}_{3}$ (3 equiv)

DCE $(0.5 \mathrm{ml})$

Ar, $140^{\circ} \mathrm{C}, 24 \mathrm{~h}$

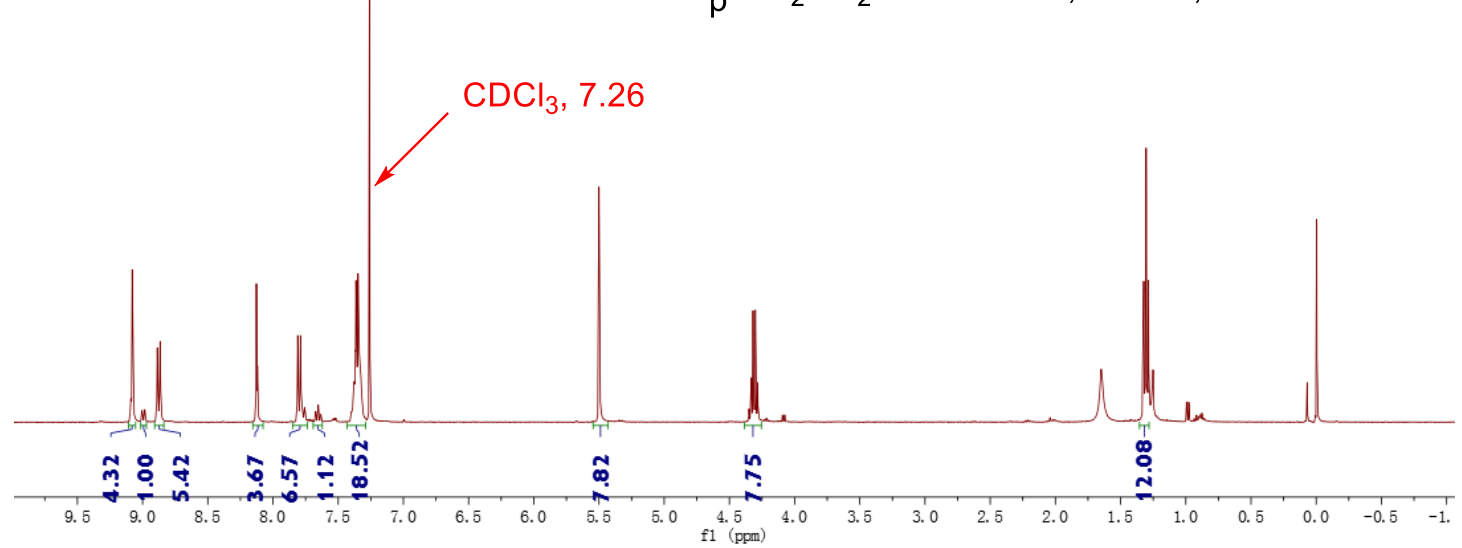

Entry 2

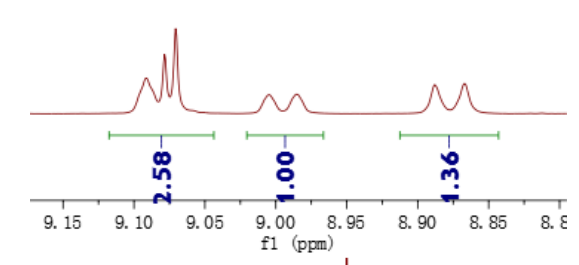

$\quad \mathrm{CDCl}_{3}, 7.26$
$\mathrm{Bn} \quad\left(3 a+3 a^{\prime}\right), 22 \%$

$\mathrm{p} / \mathrm{m}=1: 1.5$

$\left[\mathrm{RuCl}_{2}(\mathrm{p} \text {-cymene })\right]_{2}$ (5 mol\%)

$\mathrm{PivOH}(10 \mathrm{~mol} \%)$

$\mathrm{K}_{2} \mathrm{CO}_{3}$ (2 equiv)

DCE $(0.5 \mathrm{ml})$

Ar, $120^{\circ} \mathrm{C}, 24 \mathrm{~h}$

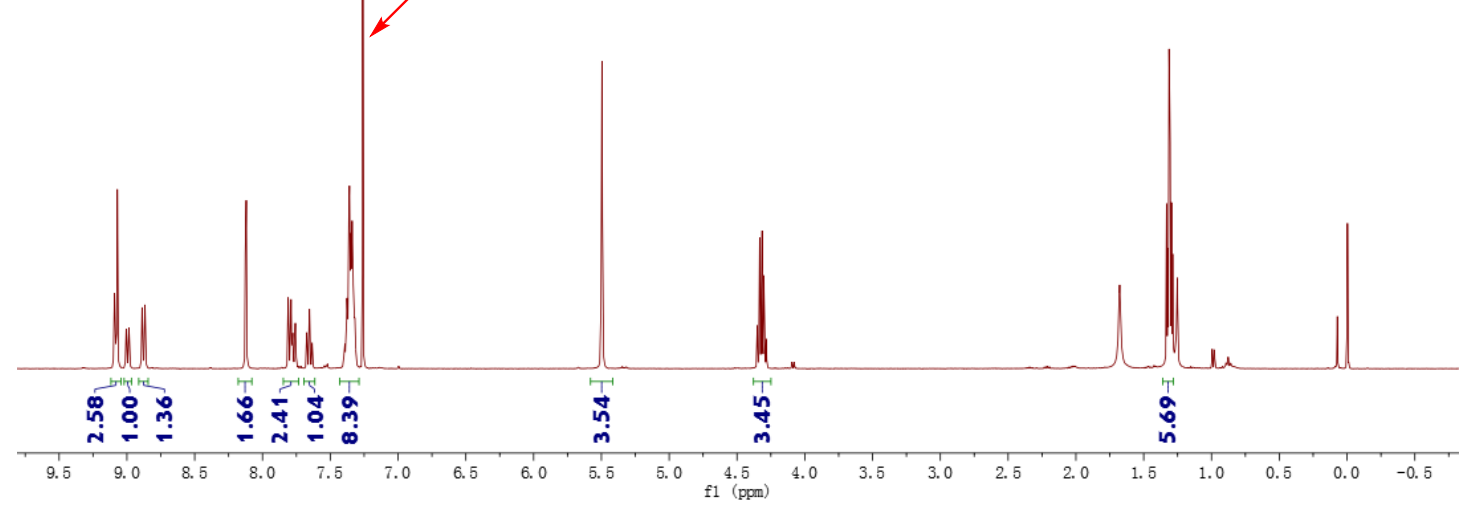


Entry 3
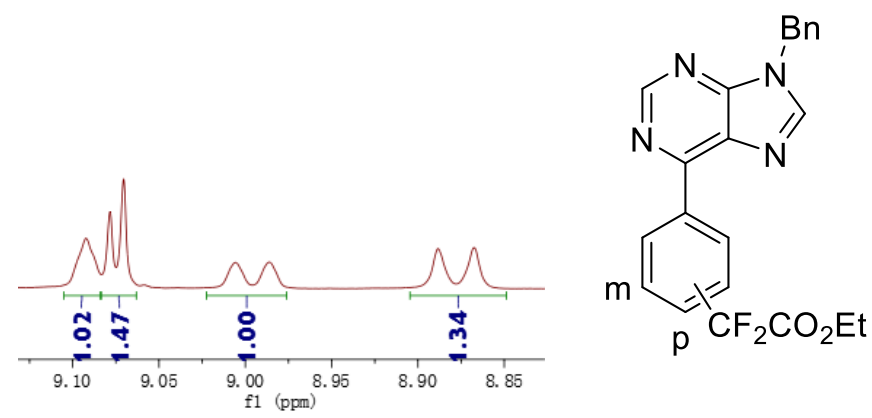

(3a+3a'), 38\%

$\mathrm{p} / \mathrm{m}=1: 1.5$

$\left[\mathrm{RuCl}_{2} \text { (p-cymene) }\right]_{2}(5 \mathrm{~mol} \%)$

L2 (10 $\mathrm{mol} \%)$

$\mathrm{K}_{2} \mathrm{CO}_{3}$ (2 equiv)

DCE $(0.5 \mathrm{ml})$

$\mathrm{Ar}, 120^{\circ} \mathrm{C}, 24 \mathrm{~h}$

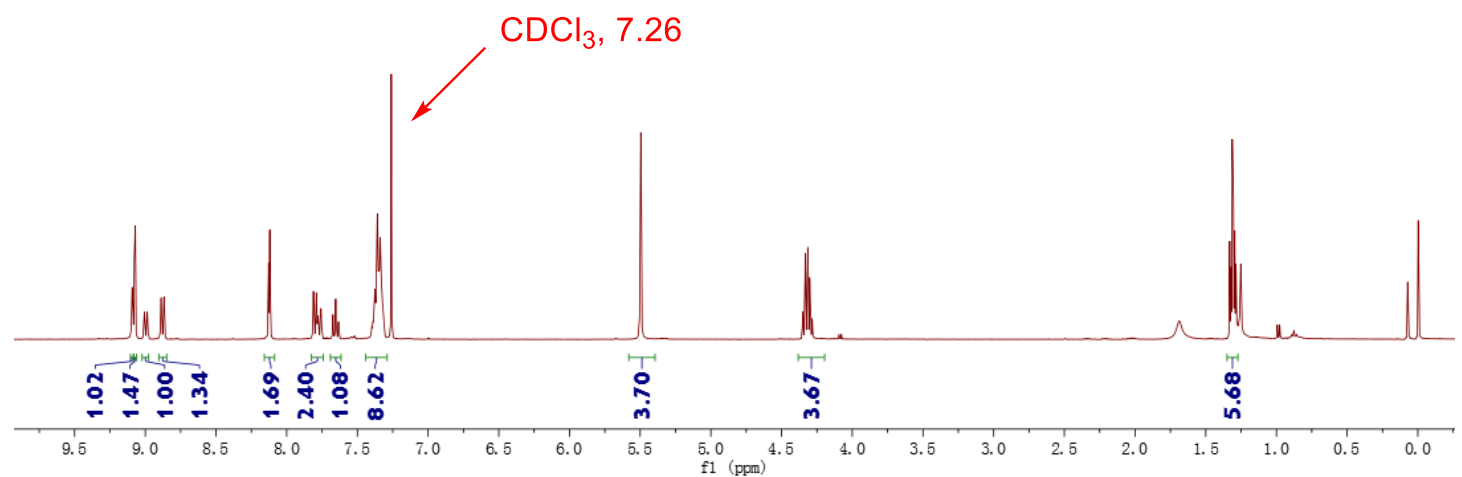

\section{Entry 4}
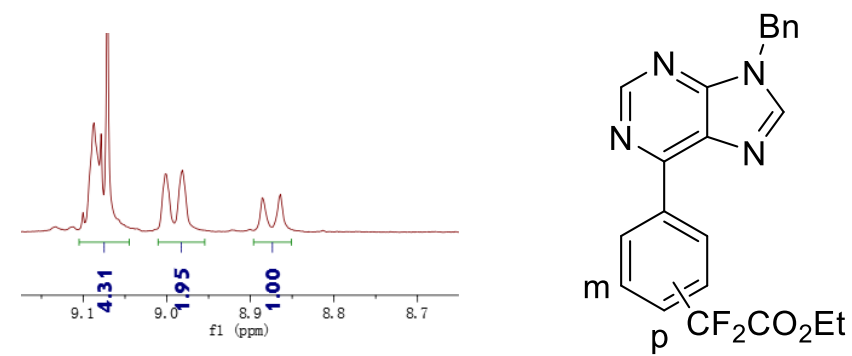

(3a+3a'), 42\%

$\mathrm{p} / \mathrm{m}=1: 3.9$

$\left[\mathrm{RuCl}_{2}(\mathrm{p} \text {-cymene })\right]_{2}(5 \mathrm{~mol} \%)$

L3 (10 mol\%)

$\mathrm{K}_{2} \mathrm{CO}_{3}$ (3 equiv)

DCE $(0.5 \mathrm{ml})$

Ar, $140^{\circ} \mathrm{C}, 24 \mathrm{~h}$

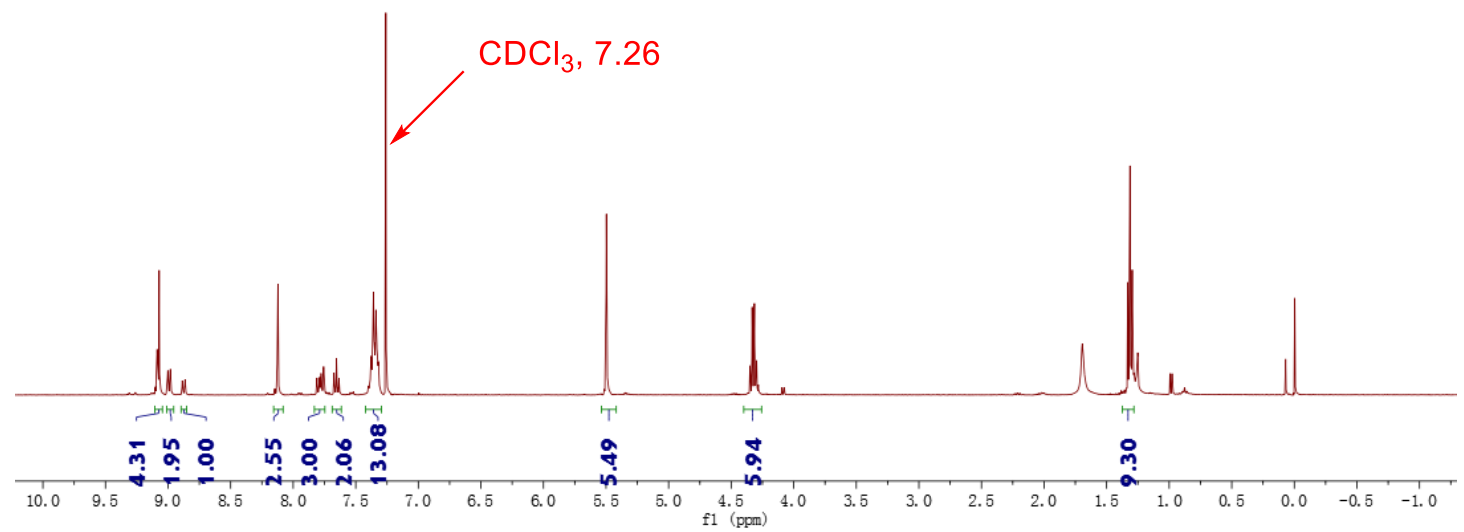


Entry 6
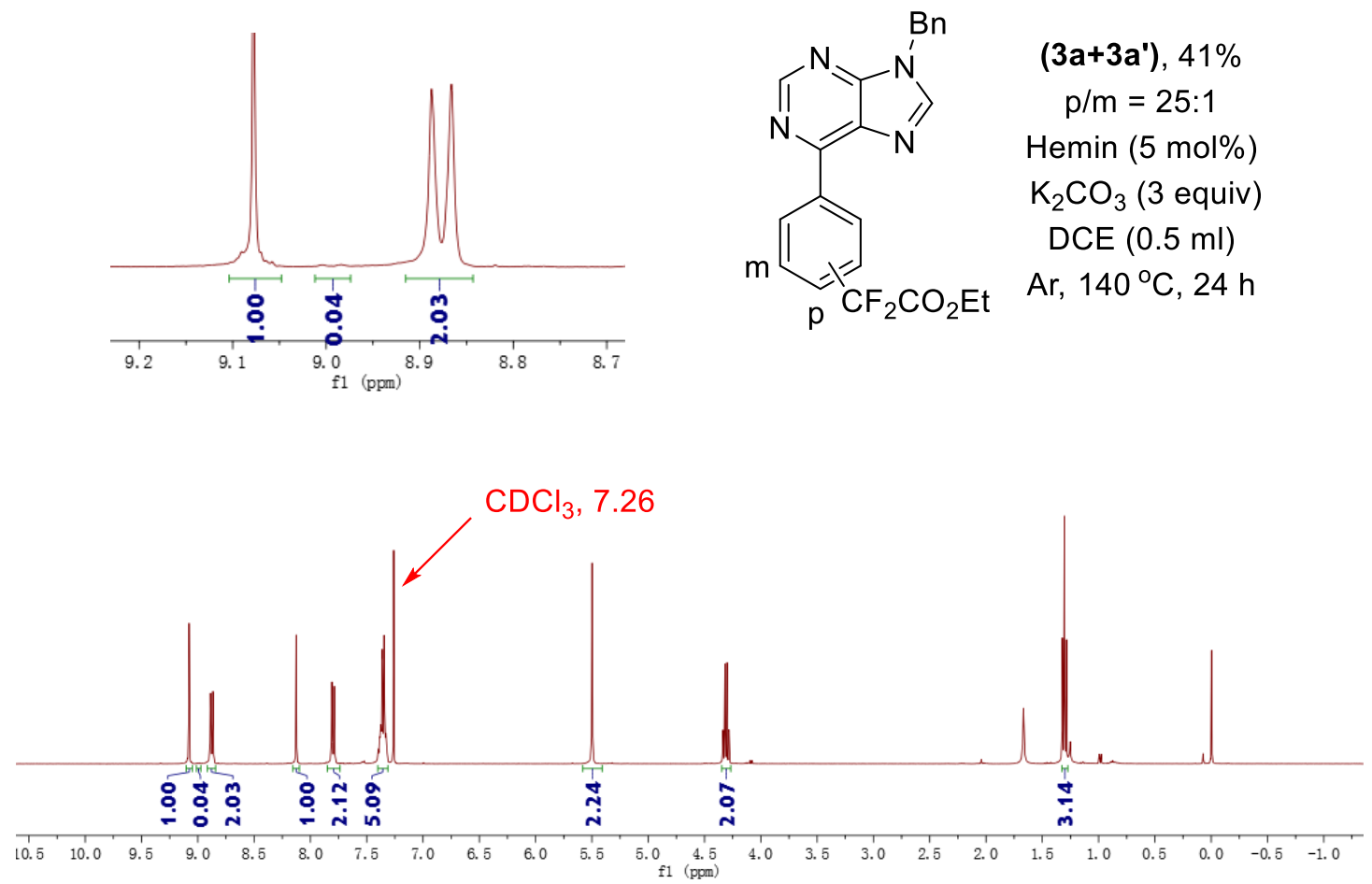

Entry 7
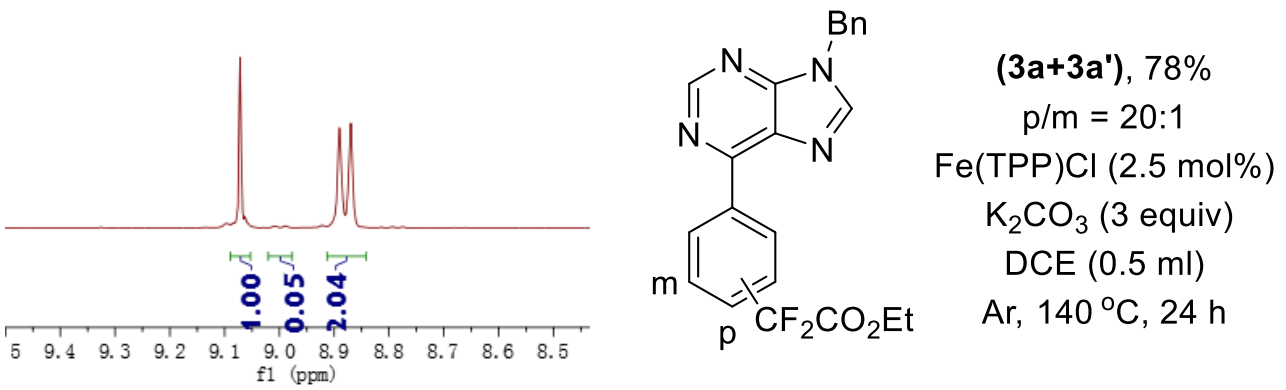

DCE $(0.5 \mathrm{ml})$

$\mathrm{Ar}, 140^{\circ} \mathrm{C}, 24 \mathrm{~h}$

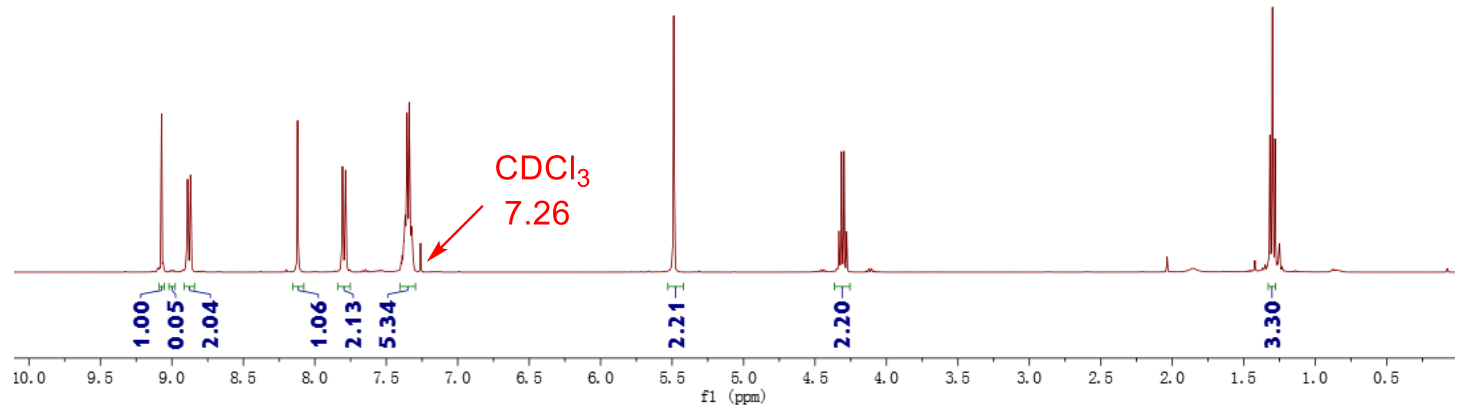


Entry 11
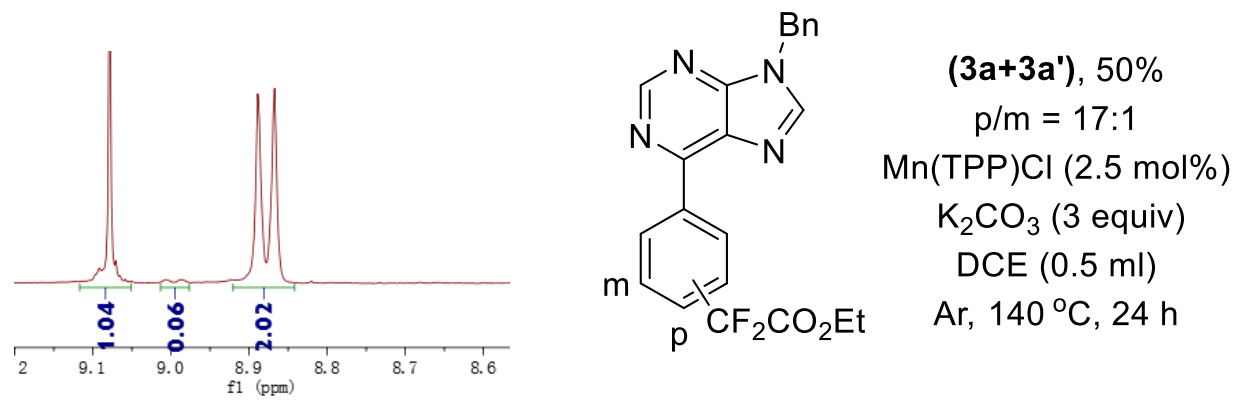

Ar, $140^{\circ} \mathrm{C}, 24 \mathrm{~h}$

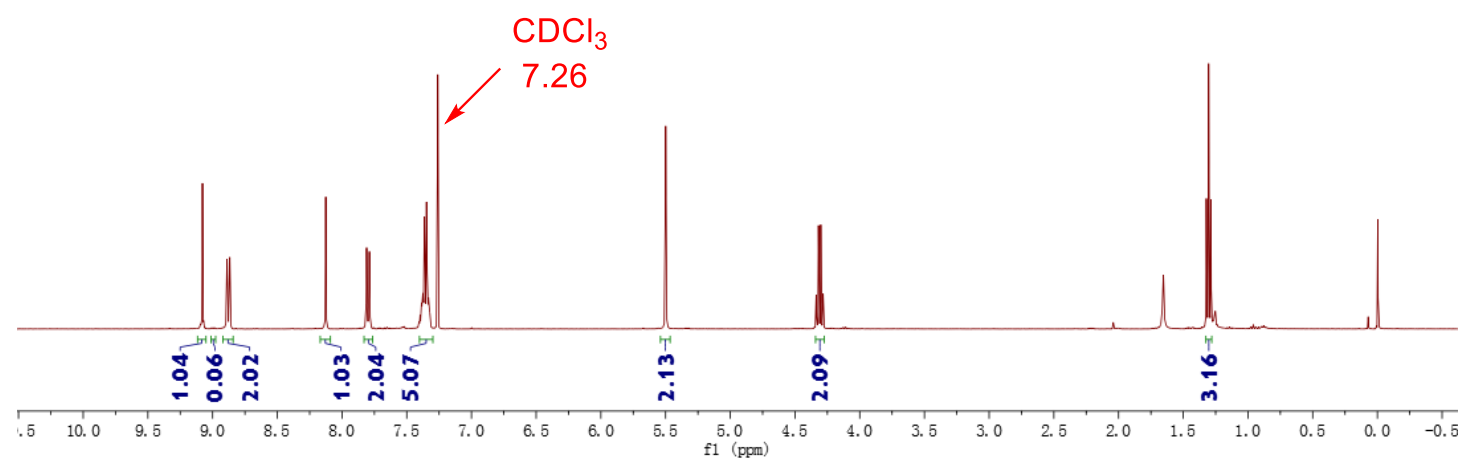




\section{Details for optimization}

Table S1. Optimization of reaction conditions. ${ }^{a}$
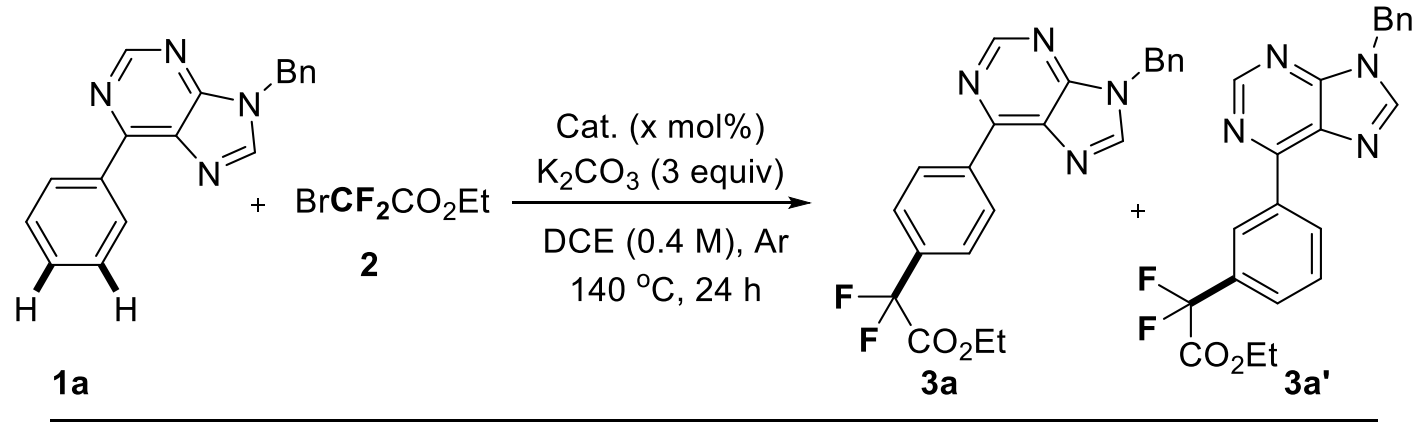

$1 \mathrm{a}$

\begin{tabular}{|c|c|c|c|c|}
\hline entry $^{a}$ & $\begin{array}{l}\text { Catalyst } \\
(\mathrm{x} \mathrm{mol} \%)\end{array}$ & Ligand & $\begin{array}{l}\left(\mathbf{3 a}+\mathbf{3} \mathbf{a}^{\prime}\right) \\
\text { Yield }(\%)^{b}\end{array}$ & $\begin{array}{c}\left(\mathbf{3 a} / \mathbf{3} \mathbf{a}^{\prime}\right) \\
(\mathrm{p} / \mathrm{m})^{c}\end{array}$ \\
\hline 1 & {$\left[\mathrm{RuCl}_{2}(\mathrm{p} \text {-cymene })\right]_{2}(5)$} & $\mathbf{L 1}$ & 51 & $2.7: 1$ \\
\hline $2^{d}$ & {$\left[\mathrm{RuCl}_{2}(\mathrm{p} \text {-cymene })\right]_{2}(5)$} & PivOH & 22 & $1: 1.5$ \\
\hline $3^{d}$ & {$\left[\mathrm{RuCl}_{2}(\mathrm{p} \text {-cymene })\right]_{2}(5)$} & $\mathbf{L} 2$ & 38 & $1: 1.5$ \\
\hline 4 & {$\left[\mathrm{RuCl}_{2}(\mathrm{p} \text {-cymene })\right]_{2}(5)$} & $\mathbf{L 3}$ & 42 & $1: 3.9$ \\
\hline 5 & {$\left[\mathrm{RuCl}_{2}(\mathrm{p} \text {-cymene })\right]_{2}(5)$} & L4 & trace & -- \\
\hline 6 & {$\left[\mathrm{Cp}^{*} \mathrm{RhCl}_{2}\right]_{2}(5)$} & -- & $\mathrm{nr}$ & -- \\
\hline 7 & {$\left[\mathrm{Cp}^{*} \mathrm{IrCl}_{2}\right]_{2}(5)$} & -- & $\mathrm{nr}$ & - \\
\hline 8 & $\mathrm{Ni}(\mathrm{acac})_{2}(10)$ & -- & $\mathrm{nr}$ & - \\
\hline 9 & $\mathrm{Co}\left(\mathrm{PPh}_{3}\right) \mathrm{Cl}_{2}(10)$ & -- & $\mathrm{nr}$ & - \\
\hline 10 & $\mathrm{FeBr}_{2}(10)$ & -- & $\mathrm{nr}$ & -- \\
\hline 11 & $\mathrm{FeCl}_{3}(10)$ & -- & $\mathrm{nr}$ & - \\
\hline 12 & $\mathrm{Fe}(\mathrm{OTs})_{3}(10)$ & -- & $\mathrm{nr}$ & - \\
\hline 13 & $\operatorname{Hemin}(5)$ & -- & 41 & $25: 1$ \\
\hline
\end{tabular}

${ }^{a}$ Reactions were performed with 1a $(0.2 \mathrm{mmol}), 2$ (1.2 mmol, $154 \mathrm{ul}, 6$ equiv ), Cat. (x mol\%), and $\mathrm{K}_{2} \mathrm{CO}_{3}$ (3 equiv) in DCE $(0.5 \mathrm{~mL}), \mathrm{Ar}, 140{ }^{\circ} \mathrm{C}, 24 \mathrm{~h} .{ }^{b}$ Yields of product after silica gel chromatography. ${ }^{c}$ The ratio of selectivity determined by ${ }^{1} \mathrm{H}$ NMR spectroscopy. ${ }^{d} \mathrm{~K}_{2} \mathrm{CO}_{3}(0.4 \mathrm{mmol}$, 2 equiv) was added at $120^{\circ} \mathrm{C}$. 
Table S2. Screening of catalyst. ${ }^{a}$

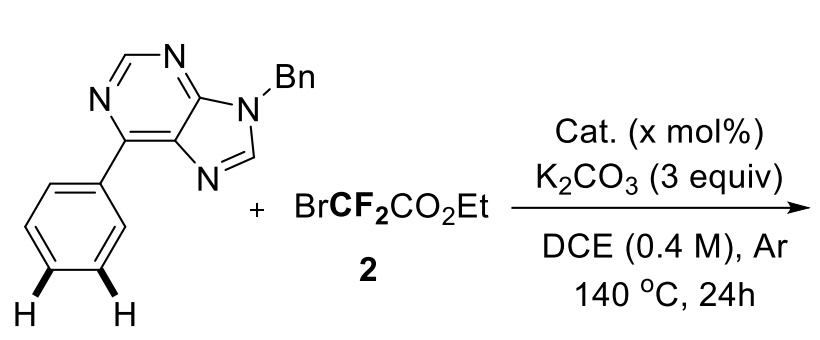

1a

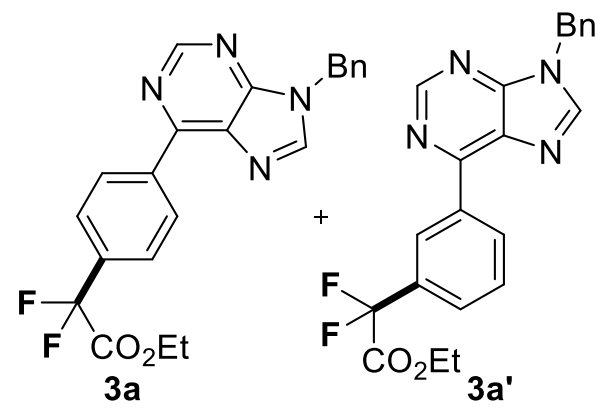

\begin{tabular}{|c|c|c|c|}
\hline entry $^{a}$ & $\begin{array}{l}\text { Catalyst } \\
(\mathrm{x} \mathrm{mol} \%)\end{array}$ & $\begin{array}{c}\left(\mathbf{3 a}+\mathbf{3} \mathbf{a}^{\prime}\right) \\
\text { Yield }(\%)^{b}\end{array}$ & $\begin{array}{c}\left(\mathbf{3 a} / \mathbf{3} \mathbf{a}^{\prime}\right) \\
(\mathrm{p} / \mathrm{m})^{c}\end{array}$ \\
\hline 1 & $\operatorname{Hemin}(5)$ & 41 & $25: 1$ \\
\hline 2 & $\mathrm{Fe}(\mathrm{TPP}) \mathrm{Cl}(2.5)$ & 78 & $20: 1$ \\
\hline 3 & $\mathrm{~T}(p$-OMe)PPFeCl (2.5) & 61 & $12: 1$ \\
\hline 4 & $\mathrm{Mn}(\mathrm{TPP}) \mathrm{Cl}(2.5)$ & 50 & $17: 1$ \\
\hline 5 & СoТРP (5) & $\mathrm{nr}$ & -- \\
\hline 6 & NiTPP (5) & $\mathrm{nr}$ & -- \\
\hline 7 & CuTPP (5) & $\mathrm{nr}$ & -- \\
\hline 8 & ZnTPP (5) & $\mathrm{nr}$ & -- \\
\hline 9 & $\operatorname{MnPc}(2.5)$ & 36 & $27: 1$ \\
\hline 10 & $\mathrm{CoPc}(5)$ & $\mathrm{nr}$ & -- \\
\hline 11 & CuPc (5) & $\mathrm{nr}$ & -- \\
\hline 12 & $\mathrm{ZnPc}(5)$ & $\mathrm{nr}$ & -- \\
\hline 13 & $\operatorname{MgPc}(5)$ & $\mathrm{nr}$ & -- \\
\hline 14 & $\operatorname{AlClPc}(5)$ & $\mathrm{nr}$ & -- \\
\hline 15 & Direct Blue 86 (5) & $\mathrm{nr}$ & -- \\
\hline
\end{tabular}

${ }^{a}$ Reactions were performed with 1a $(0.2 \mathrm{mmol}), 2$ (1.2 mmol, $154 \mathrm{ul}, 6$ equiv ), Cat. (x mol\%), and S9 
$\mathrm{K}_{2} \mathrm{CO}_{3}$ (3 equiv) in DCE $(0.5 \mathrm{~mL}), \mathrm{Ar}, 140{ }^{\circ} \mathrm{C}, 24 \mathrm{~h} .{ }^{b}$ Yields of product after silica gel chromatography. ${ }^{c}$ The ratio of selectivity determined by ${ }^{1} \mathrm{H}$ NMR spectroscopy.

Table S3. Screening of solvent. ${ }^{a}$

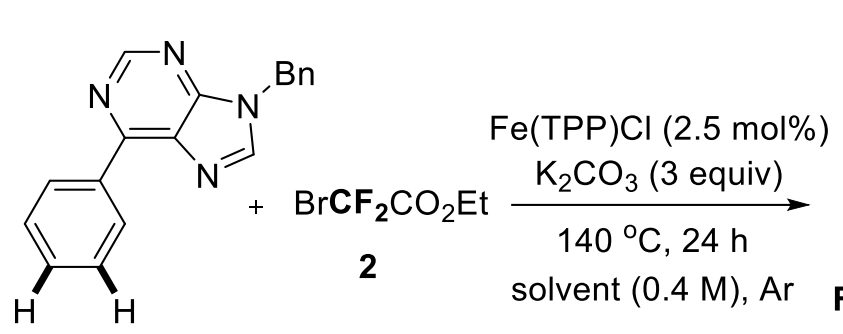

1a<smiles>CCOC(=O)c1cccc(-c2ncnc3c2ncn3Cc2ccccc2)c1</smiles>

\begin{tabular}{|c|c|c|c|}
\hline entry $^{a}$ & Solvent & 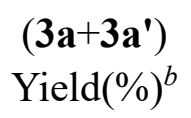 & $\begin{array}{c}\left(\mathbf{3 a} / \mathbf{3} \mathbf{a}^{\prime}\right) \\
(\mathrm{p} / \mathrm{m})^{c}\end{array}$ \\
\hline 1 & DCE & 78 & $20 / 1$ \\
\hline 2 & n-Hexane & 30 & $8 / 1$ \\
\hline 3 & HFIP & $\mathrm{nr}$ & -- \\
\hline 4 & DMF & $\mathrm{nr}$ & - \\
\hline 5 & $\mathrm{CH}_{3} \mathrm{CN}$ & $\mathrm{nr}$ & - \\
\hline 6 & DMSO & $\mathrm{nr}$ & - \\
\hline 7 & MTBE & 51 & $6 / 1$ \\
\hline 8 & THF & $<10$ & - \\
\hline 9 & 1,4-dioxane & 43 & $10 / 1$ \\
\hline 10 & toluene & $<10$ & -- \\
\hline
\end{tabular}

${ }^{a}$ Reactions were performed with 1a $(0.2 \mathrm{mmol}), 2$ (1.2 mmol, $154 \mathrm{ul}, 6$ equiv $), \mathrm{Fe}(\mathrm{Tpp}) \mathrm{Cl}(2.5$ mol\%), and $\mathrm{K}_{2} \mathrm{CO}_{3}$ (3 equiv) in solvent $(0.5 \mathrm{~mL}), \mathrm{Ar}, 140{ }^{\circ} \mathrm{C}, 24 \mathrm{~h} .{ }^{b}$ Yields of product after silica gel chromatography. ${ }^{c}$ The ratio of selectivity determined by ${ }^{1} \mathrm{H}$ NMR spectroscopy. 
Table S4. Screening of base. ${ }^{a}$

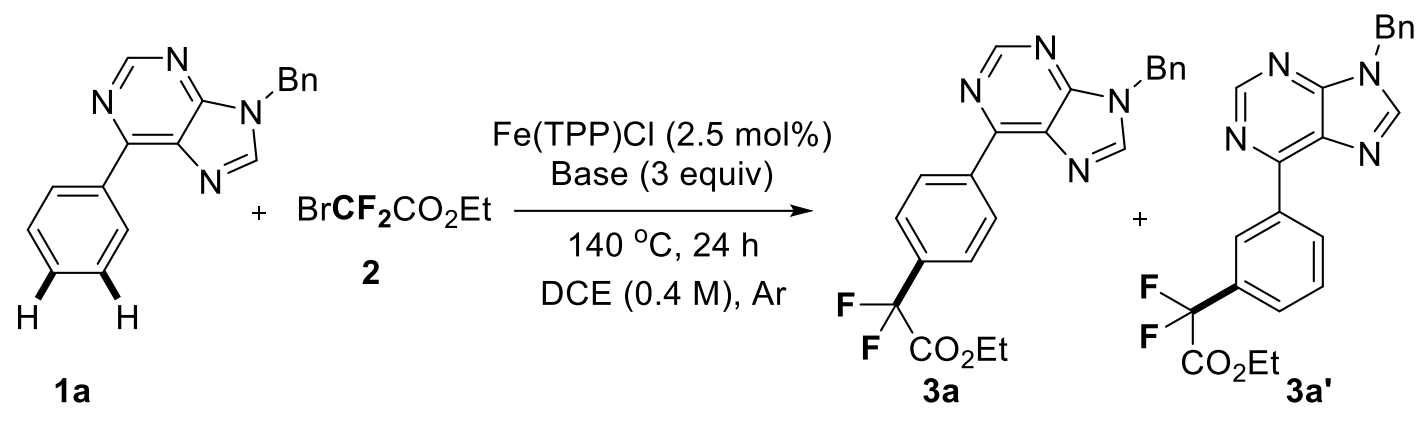

\begin{tabular}{|c|c|c|c|}
\hline entry $^{a}$ & base & 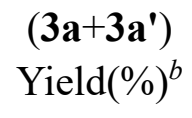 & $\begin{array}{c}\left(\mathbf{3 a} / \mathbf{3} \mathbf{a}^{\prime}\right) \\
(\mathrm{p} / \mathrm{m})^{c}\end{array}$ \\
\hline 1 & $\mathrm{~K}_{2} \mathrm{CO}_{3}$ & 78 & $20 / 1$ \\
\hline 2 & $\mathrm{KHCO}_{3}$ & 47 & $20 / 1$ \\
\hline 3 & KOAC & 21 & $9 / 1$ \\
\hline 4 & $\mathrm{Na}_{2} \mathrm{CO}_{3}$ & 52 & $6 / 1$ \\
\hline 5 & $\mathrm{NaHCO}_{3}$ & trace & -- \\
\hline 6 & $\mathrm{NaOAc}$ & $<10$ & -- \\
\hline 7 & LiOAc & trace & -- \\
\hline 8 & $\mathrm{~K}_{2} \mathrm{HPO}_{4}$ & trace & -- \\
\hline 9 & DMAP & trace & -- \\
\hline
\end{tabular}

${ }^{a}$ Reactions were performed with 1a $(0.2 \mathrm{mmol}), 2$ (1.2 mmol, $154 \mathrm{ul}, 6$ equiv $), \mathrm{Fe}(\mathrm{Tpp}) \mathrm{Cl}(2.5$ mol\%), and base (3 equiv) in DCE $(0.5 \mathrm{~mL}), \mathrm{Ar}, 140{ }^{\circ} \mathrm{C}, 24 \mathrm{~h} .{ }^{b}$ Yields of product after silica gel chromatography. ${ }^{c}$ The ratio of selectivity determined by ${ }^{1} \mathrm{H}$ NMR spectroscopy. 
5. NOESY analysis of 13
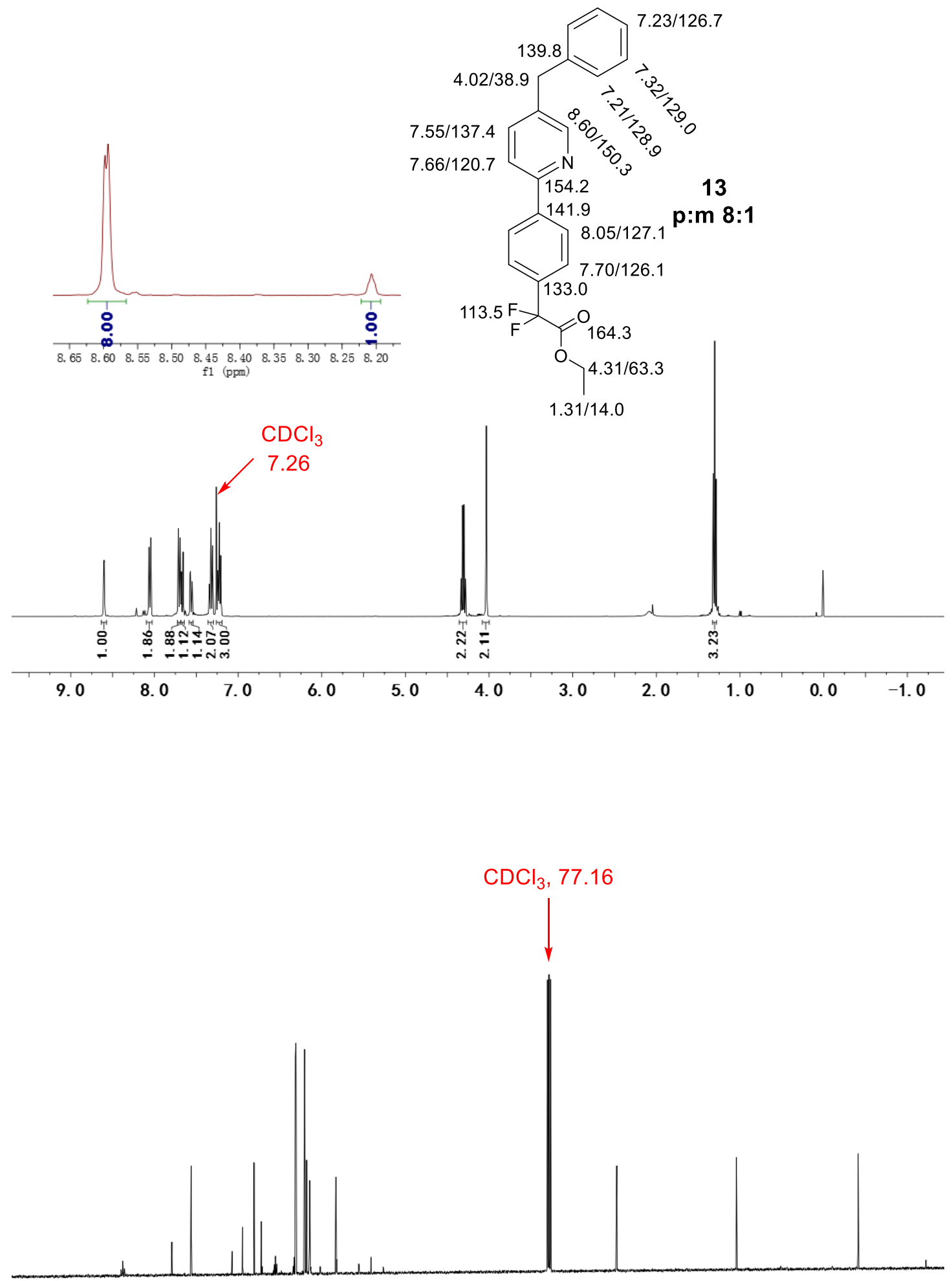

$\begin{array}{lllllllllllllllllll}180 & 170 & 160 & 150 & 140 & 130 & 120 & 110 & 100 & 90 & 80 & 70 & 60 & 50 & 40 & 30 & 20 & 10 & 0\end{array}$ 

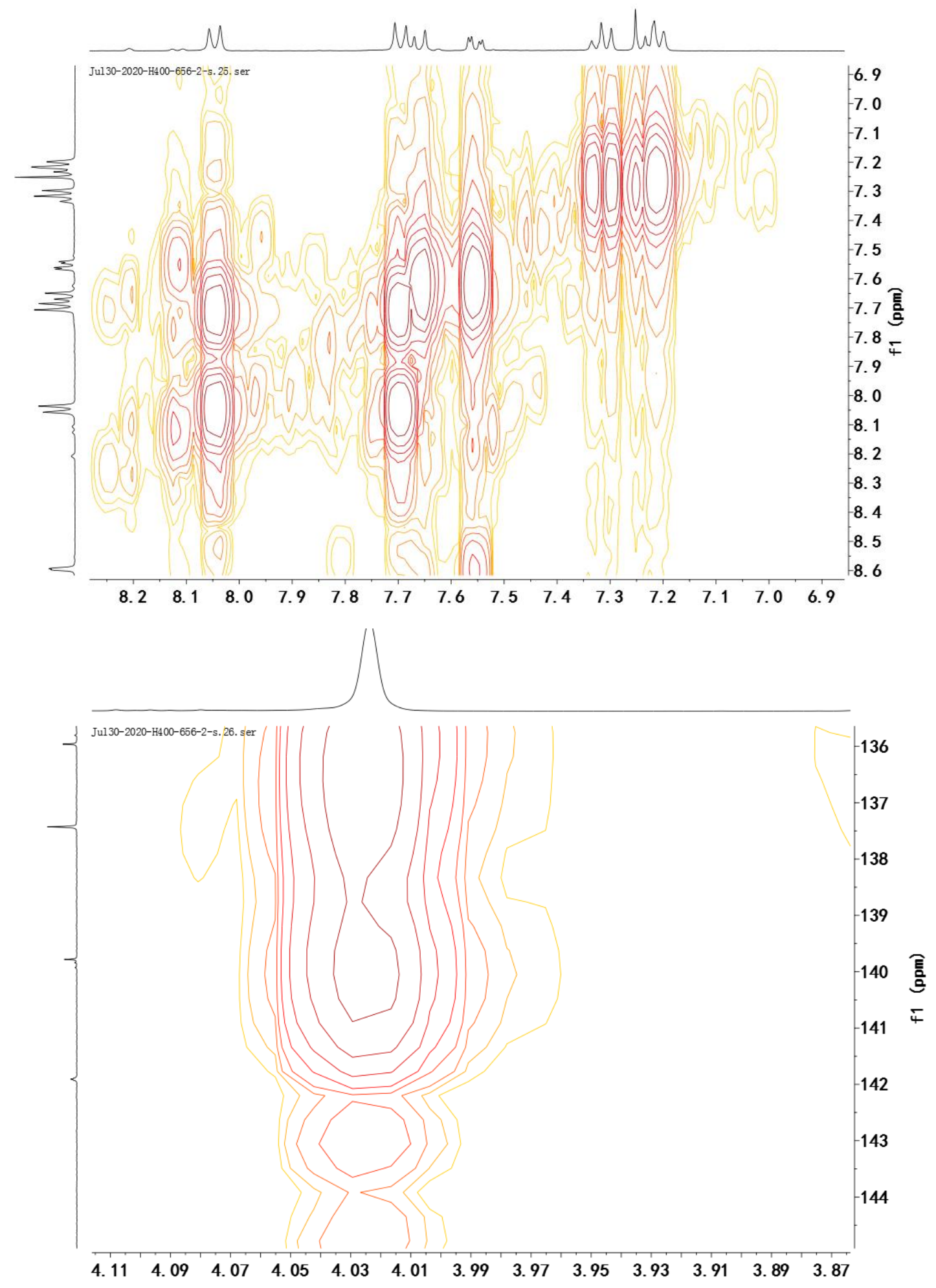


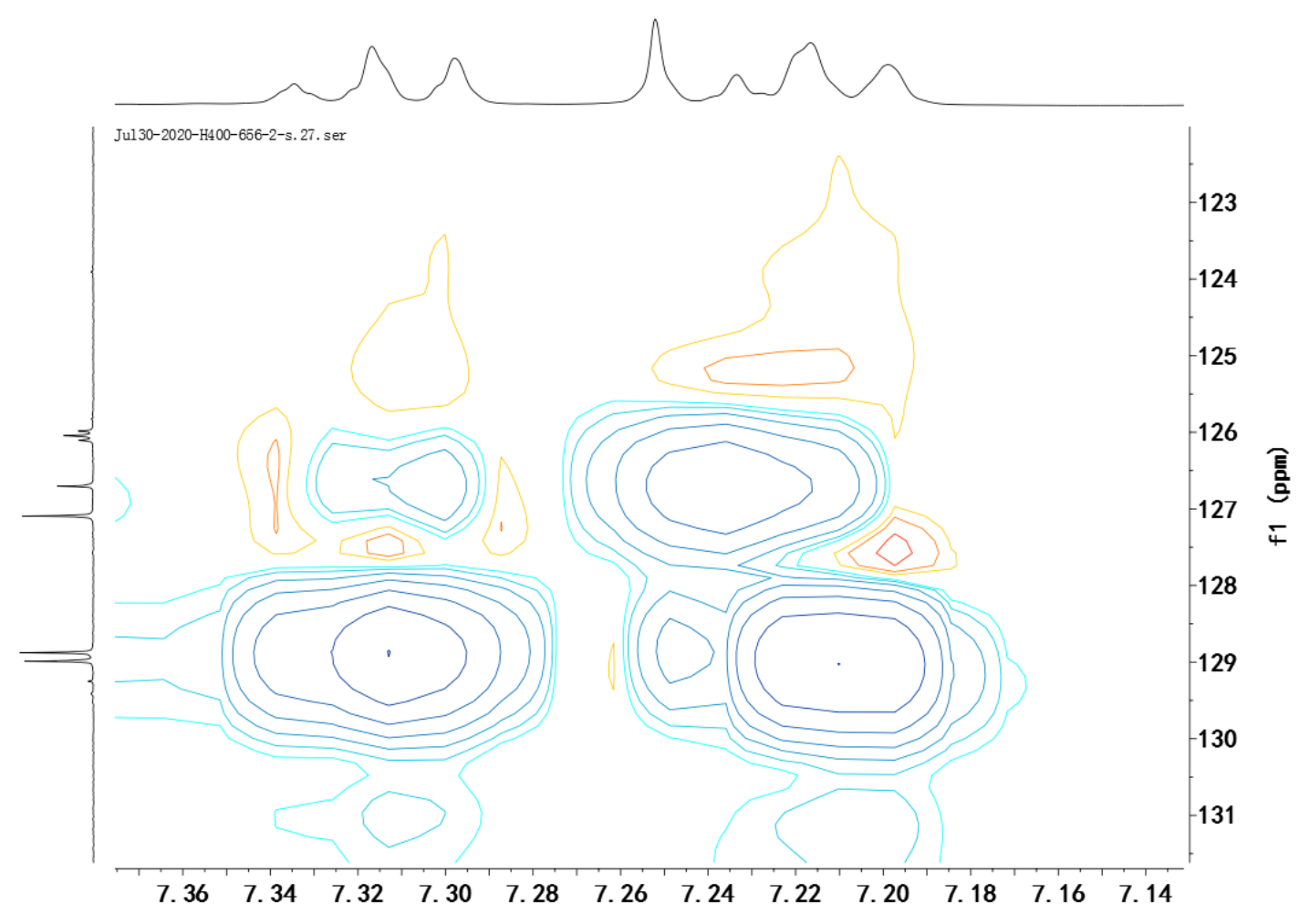

\section{Preparation of substrates}

Fenclorim and 15 were purchased from commercial sources and used without further purification. $\mathbf{1}$ and 4 were prepared according to reported methods ${ }^{1}$. 6a and $\mathbf{6 b}$ were prepared according to reported methods ${ }^{2} . \mathbf{6 c}$ were prepared according to reported methods ${ }^{3} .6 \mathbf{d}, \mathbf{6 e}$, and $\mathbf{6 f}$ were prepared according to reported methods ${ }^{4} .6 \mathrm{~g}$ and $6 \mathbf{h}$ were prepared according to reported methods ${ }^{5}$.

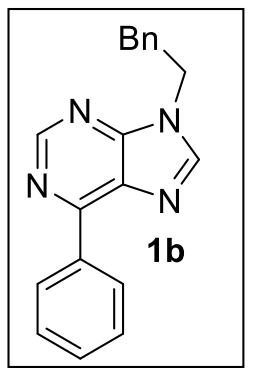

${ }^{1} \mathrm{H}$ NMR (400 MHz, Chloroform- $d$ ) $\delta 9.04(\mathrm{~s}, 1 \mathrm{H}), 8.77$ (dd, $\left.J=8.0,1.2 \mathrm{~Hz}, 2 \mathrm{H}\right), 7.70(\mathrm{~s}, 1 \mathrm{H}), 7.61-$ 7.45 (m, 3H), $7.35-7.19(\mathrm{~m}, 3 \mathrm{H}), 7.06(\mathrm{~d}, J=6.3 \mathrm{~Hz}, 2 \mathrm{H}), 4.55(\mathrm{dt}, J=6.8,3.2 \mathrm{~Hz}, 2 \mathrm{H}), 3.22(\mathrm{t}, J=$ $7.2 \mathrm{~Hz}, 2 \mathrm{H}) .{ }^{13} \mathrm{C}$ NMR $(100 \mathrm{MHz}$, Chloroform- $d$ ) $\delta 154.9,152.5,144.5,137.3,135.8,131.2,131.1,129.9$, $129.0,128.8,128.7,127.3,45.6,36.2$. 


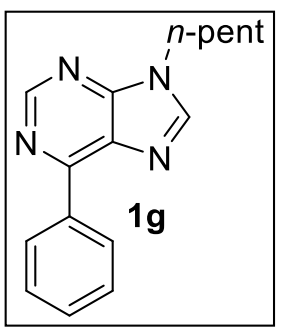

${ }^{1} \mathrm{H}$ NMR (400 MHz, Chloroform- $d$ ) $\delta 8.92(\mathrm{~s}, 1 \mathrm{H}), 8.75-8.67(\mathrm{~m}, 2 \mathrm{H}), 7.97(\mathrm{~s}, 1 \mathrm{H}), 7.51-7.33(\mathrm{~m}$, $3 \mathrm{H}), 4.11(\mathrm{t}, J=7.3 \mathrm{~Hz}, 2 \mathrm{H}), 1.78(\mathrm{q}, J=7.3 \mathrm{~Hz}, 2 \mathrm{H}), 1.30-1.11(\mathrm{~m}, 4 \mathrm{H}), 0.77(\mathrm{t}, J=7.0 \mathrm{~Hz}, 3 \mathrm{H}) .{ }^{13} \mathrm{C}$ NMR (100 MHz, Chloroform-d) $\delta$ 154.4, 152.3, 152.1, 144.2, 135.6, 130.9, 130.7, 129.6, 128.5, 43.8, 29.4, 28.6, 21.9, 13.7.

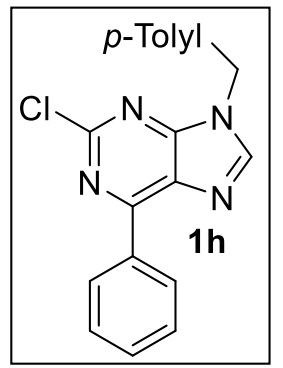

${ }^{1} \mathrm{H}$ NMR (400 MHz, Chloroform- $d$ ) $\delta 8.86-8.68(\mathrm{~m}, 2 \mathrm{H}), 8.02(\mathrm{~s}, 1 \mathrm{H}), 7.55(\mathrm{dd}, J=5.2,2.0 \mathrm{~Hz}, 3 \mathrm{H})$, $7.25-7.14$ (m, 4H), 5.39 (s, 2H), 2.35 (s, 3H). ${ }^{13} \mathrm{C}$ NMR (100 MHz, Chloroform- $d$ ) $\delta$ 156.9, 154.5, 154.4, $144.82,138.9,134.7,131.8,131.7,130.2,130.1,130.0,128.8,128.2,47.4,21.3$.

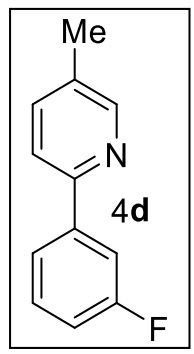

${ }^{1} \mathrm{H}$ NMR (400 MHz, Chloroform- $d$ ) $\delta 8.49$ (s, 2H), 7.80 - $7.65(\mathrm{~m}, 2 \mathrm{H}), 7.60-7.44$ (m, 2H), $7.43-7.31$ $(\mathrm{m}, 1 \mathrm{H}), 7.05(\mathrm{t}, J=8.0 \mathrm{~Hz}, 1 \mathrm{H}), 2.32(\mathrm{~s}, 3 \mathrm{H}) .{ }^{19} \mathrm{~F}$ NMR $\left(376 \mathrm{MHz}\right.$, Chloroform- $d$ ) $\delta-113.01 .{ }^{13} \mathrm{C}$ NMR $(100 \mathrm{MHz}$, Chloroform- $d) \delta 164.5,162.1,153.3\left(\mathrm{~d}, J_{C-F}=2.4 \mathrm{~Hz}\right), 150.1,141.7\left(\mathrm{~d}, J_{C-F}=7.3 \mathrm{~Hz}\right), 137.4$, $132.3,130.1\left(\mathrm{~d}, J_{C-F}=8.1 \mathrm{~Hz}\right), 122.2\left(\mathrm{~d}, J_{C-F}=2.9 \mathrm{~Hz}\right), 120.0,115.9-112.9(\mathrm{~m}), 18.11$.

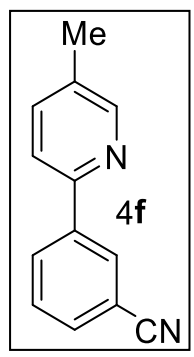

${ }^{1} \mathrm{H}$ NMR (400 MHz, Chloroform- $d$ ) $\delta 8.51(\mathrm{~s}, 1 \mathrm{H}), 8.27$ (s, 1H), 8.18 (d, J=8.0 Hz, 1H), $7.70-7.44$ (m, 4H), 2.37 (s, 3H). ${ }^{13} \mathrm{C}$ NMR (100 MHz, Chloroform- $d$ ) $\delta 152.3,150.5,140.6,137.7,133.0,131.9,130.9$, $130.4,129.6,120.0,118.9,112.9,18.3$. 


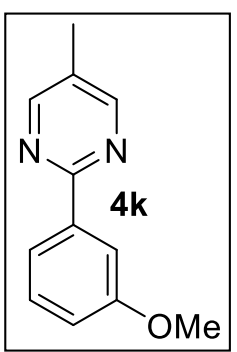

${ }^{1} \mathrm{H}$ NMR (400 MHz, Chloroform- $d$ ) $\delta 8.62(\mathrm{~s}, 2 \mathrm{H}), 8.01$ (d, J= $\left.7.7 \mathrm{~Hz}, 1 \mathrm{H}\right), 7.98-7.95$ (m, 1H), 7.38 $(\mathrm{t}, J=8.0 \mathrm{~Hz}, 1 \mathrm{H}), 7.05-6.96(\mathrm{~m}, 1 \mathrm{H}), 3.90(\mathrm{~s}, 3 \mathrm{H}), 2.32(\mathrm{~s}, 3 \mathrm{H}) .{ }^{13} \mathrm{C}$ NMR $(100 \mathrm{MHz}$, Chloroform- $d$ ) $\delta 162.4,160.1,157.5,139.2,129.7,128.5,120.5,117.1,112.4,55.5,15.6$.

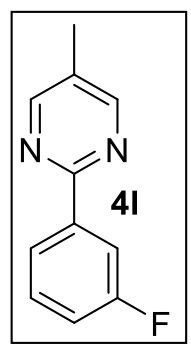

${ }^{1} \mathrm{H}$ NMR (400 MHz, Chloroform- $d$ ) $\delta 8.60(\mathrm{~d}, J=4.0 \mathrm{~Hz}, 2 \mathrm{H}), 8.19(\mathrm{~d}, J=8.0 \mathrm{~Hz}, 1 \mathrm{H}), 8.10(\mathrm{~d}, J=8.0$ $\mathrm{Hz}, 1 \mathrm{H}), 7.42$ (qd, $J=8.0,6.2,3.0 \mathrm{~Hz}, 1 \mathrm{H}), 7.13(\mathrm{t}, J=8.0 \mathrm{~Hz}, 1 \mathrm{H}), 2.32(\mathrm{~s}, 3 \mathrm{H}) .{ }^{19} \mathrm{~F} \mathrm{NMR}(376 \mathrm{MHz}$, Chloroform- $d$ ) $\delta-113.25 .{ }^{13} \mathrm{C}$ NMR (100 MHz, Chloroform- $d$ ) $\delta 164.5,162.1,161.3,157.5,140.2(\mathrm{~d}$, $\left.J_{C-F}=8.0 \mathrm{~Hz}\right), 130.1\left(\mathrm{~d}, J_{C-F}=8.0 \mathrm{~Hz}\right), 129.0,123.5\left(\mathrm{~d}, J_{C-F}=2.9 \mathrm{~Hz}\right), 116.0\left(\mathrm{dd}, J_{C-F}=243.3,22.4 \mathrm{~Hz}\right)$, 15.6 .

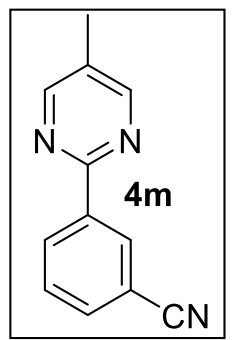

${ }^{1} \mathrm{H}$ NMR (400 MHz, Chloroform- $d$ ) $\delta 8.74(\mathrm{~s}, 1 \mathrm{H}), 8.65(\mathrm{~d}, J=4.0 \mathrm{~Hz}, 3 \mathrm{H}), 7.73(\mathrm{~d}, J=8.0 \mathrm{~Hz}, 1 \mathrm{H})$, $7.58(\mathrm{t}, J=8.0 \mathrm{~Hz}, 1 \mathrm{H}), 2.37(\mathrm{~s}, 3 \mathrm{H}) .{ }^{13} \mathrm{C}$ NMR $(100 \mathrm{MHz}$, Chloroform- $d$ ) $\delta 160.5,157.7,139.0,133.6$, $132.1,131.8,129.6,129.5,118.9,113.0,15.7$.<smiles>COc1ccc(-c2nc(Cl)cc(Cl)n2)cc1</smiles>

${ }^{1} \mathrm{H}$ NMR (400 MHz, Chloroform- $d$ ) $\delta 8.42(\mathrm{dd}, J=8.2,1.4 \mathrm{~Hz}, 2 \mathrm{H}), 7.56-7.40(\mathrm{~m}, 3 \mathrm{H}), 7.24$ (s, 1H). ${ }^{13} \mathrm{C}$ NMR (100 MHz, Chloroform- $d$ ) $\delta 165.9,162.1,135.0,132.4,129.0,128.8,118.9$. 


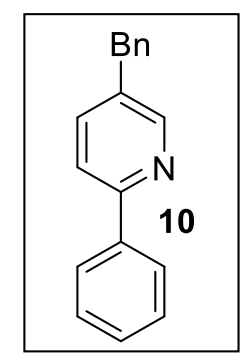

${ }^{1} \mathrm{H}$ NMR (400 MHz, Chloroform- $d$ ) $\delta 8.57(\mathrm{~d}, J=4.0 \mathrm{~Hz}, 1 \mathrm{H}), 7.99-7.91(\mathrm{~m}, 2 \mathrm{H}), 7.61(\mathrm{dd}, J=8.1$, $0.8 \mathrm{~Hz}, 1 \mathrm{H}), 7.49(\mathrm{dd}, J=8.1,2.3 \mathrm{~Hz}, 1 \mathrm{H}), 7.47-7.41(\mathrm{~m}, 2 \mathrm{H}), 7.40-7.33(\mathrm{~m}, 1 \mathrm{H}), 7.32-7.26(\mathrm{~m}$, $2 \mathrm{H}), 7.24-7.16(\mathrm{~m}, 3 \mathrm{H}), 3.98$ (s, 2H). ${ }^{13} \mathrm{C}$ NMR (100 MHz, Chloroform- $d$ ) $\delta$ 155.5, 150.0, 134.0, 139.3, 137.2, 135.0, 128.9, 128.8, 128.8, 128.8, 126.8, 126.6, 120.4, 38.8.

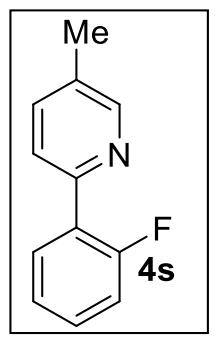

${ }^{1} \mathrm{H}$ NMR (400 MHz, Chloroform- $d$ ) $\delta 8.45(\mathrm{~d}, J=2.4 \mathrm{~Hz}, 1 \mathrm{H}), 7.85(\mathrm{tt}, J=7.8,1.3 \mathrm{~Hz}, 1 \mathrm{H}), 7.57$ (dd, $J$ $=8.2,2.3 \mathrm{~Hz}, 1 \mathrm{H}), 7.48-7.39(\mathrm{~m}, 1 \mathrm{H}), 7.27-7.21(\mathrm{~m}, 1 \mathrm{H}), 7.14(\mathrm{tt}, J=7.6,1.3 \mathrm{~Hz}, 1 \mathrm{H}), 7.07-7.00$ $(\mathrm{m}, 1 \mathrm{H}), 2.26(\mathrm{~s}, 3 \mathrm{H}) .{ }^{19} \mathrm{~F}$ NMR $\left(376 \mathrm{MHz}\right.$, Chloroform- $d$ ) $\delta-117.49 .{ }^{13} \mathrm{C}$ NMR $(100 \mathrm{MHz}$, Chloroformd) $\delta 160.5\left(\mathrm{~d}, J_{C-F}=249.3 \mathrm{~Hz}\right), 150.7\left(\mathrm{~d}, J_{C-F}=2.6 \mathrm{~Hz}\right), 150.2,136.9,132.0,130.9\left(\mathrm{~d}, J_{C-F}=3.0 \mathrm{~Hz}\right)$, $130.1\left(\mathrm{~d}, J_{C-F}=8.4 \mathrm{~Hz}\right), 127.5\left(\mathrm{~d}, J_{C-F}=11.7 \mathrm{~Hz}\right), 124.5\left(\mathrm{~d}, J_{C-F}=3.6 \mathrm{~Hz}\right), 124.0\left(\mathrm{~d}, J_{C-F}=9.2 \mathrm{~Hz}\right), 116.2$ $\left(\mathrm{d}, J_{C-F}=22.8 \mathrm{~Hz}\right), 18.3$.

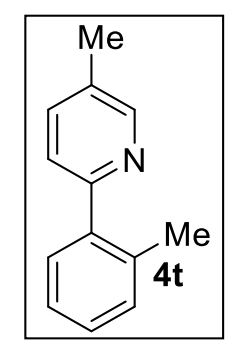

${ }^{1} \mathrm{H}$ NMR (400 MHz, Chloroform- $d$ ) $\delta 8.43(\mathrm{~s}, 1 \mathrm{H}), 7.44(\mathrm{dd}, J=8.0,2.8 \mathrm{~Hz}, 1 \mathrm{H}), 7.32-7.26(\mathrm{~m}, 1 \mathrm{H})$, $7.22-7.12(\mathrm{~m}, 4 \mathrm{H}), 2.28(\mathrm{~s}, 3 \mathrm{H}), 2.26(\mathrm{~s}, 3 \mathrm{H}) .{ }^{13} \mathrm{C}$ NMR (100 MHz, Chloroform- $d$ ) $\delta$ 157.2, 149.6, $140.4,136.8,135.8,131.1,130.7,129.7,128.1,125.9,123.6,20.4,18.2$.

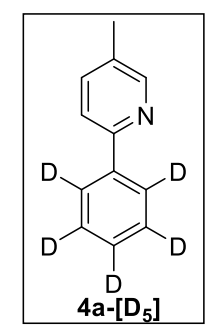

${ }^{1} \mathrm{H}$ NMR (400 MHz, Chloroform- $d$ ) $\delta 8.52(\mathrm{~s}, 1 \mathrm{H}), 7.58(\mathrm{dd}, J=7.8,3.0 \mathrm{~Hz}, 1 \mathrm{H}), 7.48(\mathrm{~d}, J=7.4 \mathrm{~Hz}$, $1 \mathrm{H}), 2.31$ (s, 3H). ${ }^{13} \mathrm{C}$ NMR (100 MHz, Chloroform- $d$ ) $\delta 154.6,150.0,139.2,137.2,131.5,128.6-$ $127.3(\mathrm{~m}), 126.6-125.7(\mathrm{~m}), 119.9,18.0$. 


\section{Procedures for preparation 3.}
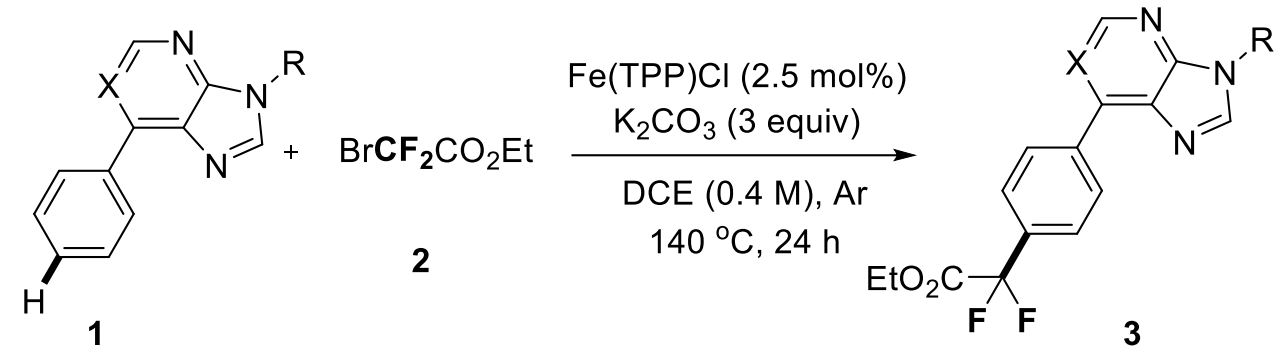

A mixture of 1 ( $0.2 \mathrm{mmol}, 1.0$ equiv), $\mathrm{BrCF}_{2} \mathrm{CO}_{2} \mathrm{Et}(1.2 \mathrm{mmol}, 154 \mu \mathrm{L}, 242.4 \mathrm{mg}, 6$ equiv), $\mathrm{Fe}(\mathrm{Tpp}) \mathrm{Cl}(3.5 \mathrm{mg}, 2.5 \mathrm{~mol} \%), \mathrm{K}_{2} \mathrm{CO}_{3}(81.6 \mathrm{mg}, 300 \mathrm{~mol} \%)$, and DCE $(0.5 \mathrm{~mL})$ in a $15 \mathrm{~mL}$ glass vial sealed under argon atmosphere was heated at $140{ }^{\circ} \mathrm{C}$ for 24 hours. The reaction mixture was cooled to room temperature and concentrated in vacuo. The resulting residue was purified by column chromatography $(\mathrm{PE} / \mathrm{EA}=10: 1)$ on silica gel to give the product 3 .

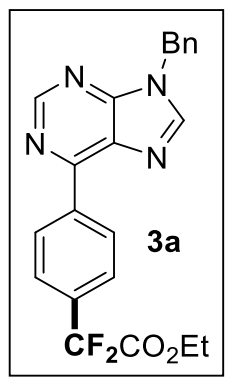

White solid. Isolated yield: $63.6 \mathrm{mg}, 78 \%$.

${ }^{1} \mathrm{H}$ NMR (400 MHz, Chloroform- $d$ ) $\delta 9.07(\mathrm{~s}, 1 \mathrm{H}), 8.88(\mathrm{~d}, J=8.2 \mathrm{~Hz}, 2 \mathrm{H}), 8.12(\mathrm{~s}, 1 \mathrm{H}), 7.80(\mathrm{~d}, J=8.4$ $\mathrm{Hz}, 2 \mathrm{H}), 7.35(\mathrm{~m}, 5 \mathrm{H}), 5.49(\mathrm{~s}, 2 \mathrm{H}), 4.31(\mathrm{q}, J=7.2 \mathrm{~Hz}, 2 \mathrm{H}), 1.30(\mathrm{t}, J=7.2 \mathrm{~Hz}, 3 \mathrm{H}) .{ }^{19} \mathrm{~F}$ NMR $(376$ MHz, Chloroform- $d$ ) $\delta-104.33 .{ }^{13} \mathrm{C}$ NMR $(100 \mathrm{MHz}$, Chloroform- $d) \delta 164.1\left(\mathrm{t}, J_{C-F}=35.1 \mathrm{~Hz}\right), 153.6$, 152.9, 152.7, 144.7, 138.4, 135.2, $134.9\left(\mathrm{t}, J_{C-F}=25.76 \mathrm{~Hz}\right), 131.3,130.1,129.3,128.8,128.0,125.9(\mathrm{t}$, $\left.J_{C-F}=6.1 \mathrm{~Hz}\right), 113.4\left(\mathrm{t}, J_{C-F}=253.5 \mathrm{~Hz}\right), 63.4,47.5,14.0$. HRMS Calcd for $\mathrm{C}_{22} \mathrm{H}_{18} \mathrm{~F}_{2} \mathrm{~N}_{4} \mathrm{O}_{2}\left[\mathrm{M}+\mathrm{H}^{+}\right]$: 409.1471, Found: 409.1466.<smiles>CCOC(=O)c1ccc(-c2ncnc3c2ncn3Cc2ccc(C(F)(F)F)cc2)cc1</smiles>

White solid. Isolated yield: $52.3 \mathrm{mg}, 62 \%$.

${ }^{1} \mathrm{H}$ NMR $(400 \mathrm{MHz}$, Chloroform- $d$ ) $\delta 9.06(\mathrm{~s}, 1 \mathrm{H}), 8.86(\mathrm{~d}, J=8.4 \mathrm{~Hz}, 2 \mathrm{H}), 7.79(\mathrm{~d}, J=8.6 \mathrm{~Hz}, 2 \mathrm{H})$, $7.72(\mathrm{~s}, 1 \mathrm{H}), 7.30-7.26(\mathrm{~m}, 1 \mathrm{H}), 7.26-7.21(\mathrm{~m}, 2 \mathrm{H}), 7.06(\mathrm{dd}, J=7.6,1.4 \mathrm{~Hz}, 2 \mathrm{H}), 4.57(\mathrm{t}, J=6.8 \mathrm{~Hz}$, $2 \mathrm{H}), 4.30(\mathrm{q}, J=7.2 \mathrm{~Hz}, 2 \mathrm{H}), 3.23(\mathrm{t}, J=6.8 \mathrm{~Hz}, 2 \mathrm{H}), 1.30(\mathrm{t}, J=7.2 \mathrm{~Hz}, 3 \mathrm{H}) .{ }^{19} \mathrm{~F}$ NMR $(376 \mathrm{MHz}$, Chloroform- $d$ ) $\delta-104.35 .{ }^{13} \mathrm{C}$ NMR $\left(100 \mathrm{MHz}\right.$, Chloroform- $d$ ) $\delta 164.11\left(\mathrm{t}, J_{C-F}=35.2 \mathrm{~Hz}\right), 153.4,152.7$, 
$152.5,145.0,138.4,137.2,134.9\left(\mathrm{t}, J_{C-F}=25.6 \mathrm{~Hz}\right), 131.4,130.1,129.1,128.8,127.4,125.9\left(\mathrm{t}, J_{C-F}=\right.$ $6.1 \mathrm{~Hz}), 113.4\left(\mathrm{t}, J_{C-F}=252.4 \mathrm{~Hz}\right), 63.4,45.7,36.1,14.0$. HRMS Calcd for $\mathrm{C}_{23} \mathrm{H}_{20} \mathrm{~F}_{2} \mathrm{~N}_{4} \mathrm{O}_{2}\left[\mathrm{M}+\mathrm{H}^{+}\right]$: 423.1627, Found: 423.1624<smiles>CCOC(=O)C(F)(F)c1ccc(-c2ncnc3c2ncn3CC)cc1</smiles>

White solid. Isolated yield: $68.4 \mathrm{mg}, 81 \%$.

${ }^{1} \mathrm{H}$ NMR (400 MHz, Chloroform- $d$ ) $\delta 9.07$ (s, 1H), $8.94-8.82(\mathrm{~m}, 2 \mathrm{H}), 8.10(\mathrm{~s}, 1 \mathrm{H}), 7.79$ (d, J = 8.4 Hz, $2 \mathrm{H}), 7.23(\mathrm{~d}, J=8.2 \mathrm{~Hz}, 2 \mathrm{H}), 7.17(\mathrm{~d}, J=8.0 \mathrm{~Hz}, 2 \mathrm{H}), 5.44(\mathrm{~s}, 2 \mathrm{H}), 4.31(\mathrm{q}, J=7.2 \mathrm{~Hz}, 2 \mathrm{H}), 2.33$ (s, $3 \mathrm{H}), 1.30(\mathrm{t}, J=7.2 \mathrm{~Hz}, 3 \mathrm{H}) .{ }^{19} \mathrm{~F}$ NMR $\left(376 \mathrm{MHz}\right.$, Chloroform- $d$ ) $\delta-104.33 .{ }^{13} \mathrm{C} \mathrm{NMR}(100 \mathrm{MHz}$, Chloroform- $d) \delta 164.1\left(\mathrm{t}, J_{C-F}=35.1 \mathrm{~Hz}\right), 153.5,152.9,152.7,144.7,138.7,138.4,134.9\left(\mathrm{t}, J_{C-F}=25.6\right.$ $\mathrm{Hz}), 132.1,131.4,130.1,130.0,128.0,125.9\left(\mathrm{t}, J_{C-F}=6.1 \mathrm{~Hz}\right), 113.4$ (t, $\left.J_{C-F}=252.4 \mathrm{~Hz}\right), 63.4,47.3$, 21.3, 14.0. HRMS Calcd for $\mathrm{C}_{23} \mathrm{H}_{20} \mathrm{~F}_{2} \mathrm{~N}_{4} \mathrm{O}_{2}\left[\mathrm{M}+\mathrm{H}^{+}\right]$: 423.1627 , Found: 423.1622<smiles>CCOC(=O)c1ccc(-c2ccnc3c2ncn3Cc2ccccc2)cc1</smiles>

White solid. Isolated yield: $41.5 \mathrm{mg}, 51 \%$.

${ }^{1} \mathrm{H}$ NMR (400 MHz, Chloroform- $d$ ) $\delta 8.51(\mathrm{~d}, J=4.0 \mathrm{~Hz}, 1 \mathrm{H}), 8.20(\mathrm{~d}, J=8.0 \mathrm{~Hz}, 2 \mathrm{H}), 8.10(\mathrm{~s}, 1 \mathrm{H})$, $7.78(\mathrm{~d}, J=8.4 \mathrm{~Hz}, 2 \mathrm{H}), 7.44(\mathrm{~d}, J=4.0 \mathrm{~Hz}, 1 \mathrm{H}), 7.39-7.28(\mathrm{~m}, 5 \mathrm{H}), 5.53(\mathrm{~s}, 2 \mathrm{H}), 4.31(\mathrm{q}, J=7.2 \mathrm{~Hz}$, $2 \mathrm{H}), 1.32(\mathrm{t}, J=7.2 \mathrm{~Hz}, 3 \mathrm{H}) .{ }^{19} \mathrm{~F}$ NMR $\left(376 \mathrm{MHz}\right.$, Chloroform- $d$ ) $\delta-104.09 .{ }^{13} \mathrm{C}$ NMR $(100 \mathrm{MHz}$, Chloroform-d) $\delta 164.2\left(\mathrm{t}, J_{C-F}=35.2 \mathrm{~Hz}\right), 148.1,145.0,144.1,138.8,138.7,136.0,133.3,133.0,129.6$, 129.2, 128.5, 127.9, $126.1\left(\mathrm{t}, J_{C-F}=6.1 \mathrm{~Hz}\right), 116.8,113.5\left(\mathrm{t}, J_{C-F}=253.1 \mathrm{~Hz}\right), 63.4,47.4,14.0$. HRMS Calcd for $\mathrm{C}_{23} \mathrm{H}_{19} \mathrm{~F}_{2} \mathrm{~N}_{3} \mathrm{O}_{2}\left[\mathrm{M}+\mathrm{H}^{+}\right]$: 408.1518, Found: 408.1510<smiles>CCOC(=O)C(F)(F)c1ccc(-c2ncnc3c2ncn3C)cc1</smiles>

White solid. Isolated yield: $41.8 \mathrm{mg}, 63 \%$. 
${ }^{1} \mathrm{H}$ NMR (400 MHz, Chloroform- $d$ ) $\delta 9.05(\mathrm{~s}, 1 \mathrm{H}), 8.87$ (d, $\left.J=8.2 \mathrm{~Hz}, 2 \mathrm{H}\right), 8.13(\mathrm{~s}, 1 \mathrm{H}), 7.80(\mathrm{~d}, J=8.4$ $\mathrm{Hz}, 2 \mathrm{H}), 4.31(\mathrm{q}, J=7.2 \mathrm{~Hz}, 2 \mathrm{H}), 3.96(\mathrm{~s}, 3 \mathrm{H}), 1.30$ (t, $J=7.2 \mathrm{~Hz}, 3 \mathrm{H}) .{ }^{19} \mathrm{~F}$ NMR $(376 \mathrm{MHz}$, Chloroformd) $\delta$-104.36. ${ }^{13} \mathrm{C}$ NMR $(100 \mathrm{MHz}$, Chloroform- $d) \delta 164.1\left(\mathrm{t}, J_{C-F}=35.0 \mathrm{~Hz}\right), 153.5,153.2,152.6,145.4$, $138.4,135.0\left(\mathrm{t}, J_{C-F}=25.5 \mathrm{~Hz}\right), 131.3,130.1,125.9\left(\mathrm{t}, J_{C-F}=6.1 \mathrm{~Hz}\right), 113.4\left(\mathrm{t}, J_{C-F}=252.3 \mathrm{~Hz}\right), 63.4$, 30.1, 14.0. HRMS Calcd for $\mathrm{C}_{16} \mathrm{H}_{14} \mathrm{~F}_{2} \mathrm{~N}_{4} \mathrm{O}_{2}\left[\mathrm{M}+\mathrm{H}^{+}\right]$: 333.1158, Found: 333.1161 .<smiles>CCOC(=O)c1ccc(-c2ncnc3c2ncn3C(C)C)cc1</smiles>

Yellow oil. Isolated yield: $51.1 \mathrm{mg}, 71 \%$.

${ }^{1} \mathrm{H}$ NMR (400 MHz, Chloroform- $d$ ) $\delta 9.03(\mathrm{~s}, 1 \mathrm{H}), 8.86(\mathrm{~d}, J=8.4 \mathrm{~Hz}, 2 \mathrm{H}), 8.20(\mathrm{~s}, 1 \mathrm{H}), 7.79(\mathrm{~d}, J=8.4$ $\mathrm{Hz}, 2 \mathrm{H}), 4.99$ (p, $J=6.8 \mathrm{~Hz}, 1 \mathrm{H}), 4.30(\mathrm{q}, J=7.2 \mathrm{~Hz}, 2 \mathrm{H}), 1.68(\mathrm{~d}, J=6.8 \mathrm{~Hz}, 6 \mathrm{H}), 1.30$ (t, $J=7.2 \mathrm{~Hz}$, $3 \mathrm{H}) .{ }^{19} \mathrm{~F}$ NMR (376 MHz, Chloroform- $d$ ) $\delta-104.34 .{ }^{13} \mathrm{C}$ NMR $\left(100 \mathrm{MHz}\right.$, Chloroform- $d$ ) $\delta 164.1\left(\mathrm{t}, J_{C-}\right.$ $F=35.1 \mathrm{~Hz}), 153.4,152.5,152.2,142.6,138.5,134.8\left(\mathrm{t}, J_{C-F}=25.6 \mathrm{~Hz}\right), 131.8,130.1,125.9\left(\mathrm{t}, J_{C-F}=\right.$ $6.1 \mathrm{~Hz}), 113.4\left(\mathrm{t}, J_{C-F}=252.3 \mathrm{~Hz}\right), 63.4,47.6,22.7,14.0$. HRMS Calcd for $\mathrm{C}_{18} \mathrm{H}_{18} \mathrm{~F}_{2} \mathrm{~N}_{4} \mathrm{O}_{2}\left[\mathrm{M}+\mathrm{H}^{+}\right]$: 361.1471, Found: 361.1467.<smiles>CCOC(=O)c1ccc(-c2ncnc3c2ncn3COc2ccccc2)cc1</smiles>

Yellow oil. Isolated yield: $62.1 \mathrm{mg}, 80 \%$.

${ }^{1} \mathrm{H}$ NMR (400 MHz, Chloroform- $d$ ) $\delta 9.01(\mathrm{~s}, 1 \mathrm{H}), 8.86(\mathrm{~d}, J=8.6 \mathrm{~Hz}, 2 \mathrm{H}), 8.12(\mathrm{~s}, 1 \mathrm{H}), 7.78(\mathrm{~d}, J=8.6$ $\mathrm{Hz}, 2 \mathrm{H}), 4.32-4.25$ (m, 4H), 1.93 (p, J=7.2 Hz, 2H), $1.37-1.30(\mathrm{~m}, 4 \mathrm{H}), 1.28$ (t, J= 7.2 Hz, 3H), 0.87 $(\mathrm{t}, J=7.0 \mathrm{~Hz}, 3 \mathrm{H}) .{ }^{19} \mathrm{~F}$ NMR (376 MHz, Chloroform- $d$ ) $\delta-104.34 .{ }^{13} \mathrm{C}$ NMR $(100 \mathrm{MHz}$, Chloroform- $d$ ) $\delta 164.1\left(\mathrm{t}, J_{C-F}=35.1 \mathrm{~Hz}\right), 153.3,152.8,152.4,144.9,138.4,134.8\left(\mathrm{t}, J_{C-F}=25.6 \mathrm{~Hz}\right), 131.4,130.0$, $125.8\left(\mathrm{t}, J_{C-F}=6.0 \mathrm{~Hz}\right), 113.4\left(\mathrm{t}, J_{C-F}=253.5 \mathrm{~Hz}\right), 63.3,44.1,29.7,28.9,22.2$, 13.9. HRMS Calcd for $\mathrm{C}_{20} \mathrm{H}_{22} \mathrm{~F}_{2} \mathrm{~N}_{4} \mathrm{O}_{2}\left[\mathrm{M}+\mathrm{H}^{+}\right]$: 389.1784, Found: 389.1780.<smiles>CCOC(=O)c1ccc(-c2nc(Cl)nc3c2ncn3CC)cc1</smiles> 
White solid. Isolated yield: $68.4 \mathrm{mg}, 75 \%$.

${ }^{1} \mathrm{H}$ NMR $(400 \mathrm{MHz}$, Chloroform- $d$ ) $\delta 8.85(\mathrm{~d}, J=8.4 \mathrm{~Hz}, 2 \mathrm{H}), 8.05(\mathrm{~s}, 1 \mathrm{H}), 7.77(\mathrm{~d}, J=8.4 \mathrm{~Hz}, 2 \mathrm{H})$, $7.23(\mathrm{~d}, J=8.2 \mathrm{~Hz}, 2 \mathrm{H}), 7.18(\mathrm{~d}, J=8.0 \mathrm{~Hz}, 2 \mathrm{H}), 5.39(\mathrm{~s}, 2 \mathrm{H}), 4.31(\mathrm{q}, J=7.2 \mathrm{~Hz}, 2 \mathrm{H}), 2.34(\mathrm{~s}, 3 \mathrm{H})$, $1.30(\mathrm{t}, J=7.2 \mathrm{~Hz}, 3 \mathrm{H}) .{ }^{19} \mathrm{~F}$ NMR $\left(376 \mathrm{MHz}\right.$, Chloroform- $d$ ) $\delta-104.41 .{ }^{13} \mathrm{C}$ NMR (100 MHz, Chloroformd) $\delta 164.0\left(\mathrm{t}, J_{C-F}=35.1 \mathrm{~Hz}\right), 155.2,154.5,154.5,145.4,139.0,137.2,135.6\left(\mathrm{t}, J_{C-F}=25.6 \mathrm{~Hz}\right), 131.6$, 130.3, 130.0, 128.2, $126.0\left(\mathrm{t}, J_{C-F}=6.1 \mathrm{~Hz}\right), 113.3\left(\mathrm{t}, J_{C-F}=252.5 \mathrm{~Hz}\right), 63.4,47.5,21.3$, 14.0. HRMS Calcd for $\mathrm{C}_{23} \mathrm{H}_{19} \mathrm{ClF}_{2} \mathrm{~N}_{4} \mathrm{O}_{2}\left[\mathrm{M}+\mathrm{H}^{+}\right]$: 457.1237, Found: 457.1240

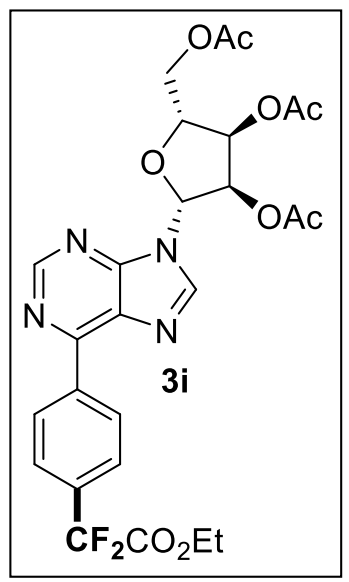

Yellow oil. Isolated yield: $66.8 \mathrm{mg}, 58 \%$.

${ }^{1} \mathrm{H}$ NMR $(400 \mathrm{MHz}$, Chloroform- $d$ ) $\delta 9.05(\mathrm{~s}, 1 \mathrm{H}), 8.86(\mathrm{~d}, J=8.0 \mathrm{~Hz}, 2 \mathrm{H}), 8.30(\mathrm{~s}, 1 \mathrm{H}), 7.80(\mathrm{~d}, J=8.0$ $\mathrm{Hz}, 2 \mathrm{H}), 6.30(\mathrm{~d}, J=4.0 \mathrm{~Hz}, 1 \mathrm{H}), 6.01(\mathrm{t}, J=6.0 \mathrm{~Hz}, 1 \mathrm{H}), 5.71(\mathrm{t}, J=6.0 \mathrm{~Hz}, 1 \mathrm{H}), 4.52-4.45(\mathrm{~m}, 3 \mathrm{H})$, $4.40(\mathrm{dd}, J=13.0,6.0 \mathrm{~Hz}, 1 \mathrm{H}), 4.31(\mathrm{q}, J=8.0 \mathrm{~Hz}, 2 \mathrm{H}), 2.17$ (s, 3H), 2.14 (s, 3H), 2.09 (s, 3H), 1.31 (t, $J=8.0 \mathrm{~Hz}, 3 \mathrm{H}) .{ }^{19} \mathrm{~F}$ NMR $\left(376 \mathrm{MHz}\right.$, Chloroform- $d$ ) $\delta-104.38 .{ }^{13} \mathrm{C}$ NMR $(100 \mathrm{MHz}$, Chloroform- $d$ ) $\delta$ $170.5,169.7,169.5,164.1\left(\mathrm{t}, J_{C-F}=36.4 \mathrm{~Hz}\right), 154.2,152.9,152.4,143.2,138.1,135.2\left(\mathrm{t}, J_{C-F}=25.6 \mathrm{~Hz}\right)$, $132.1,130.2,126.0\left(\mathrm{t}, J_{C-F}=5.1 \mathrm{~Hz}\right), 113.4\left(\mathrm{t}, J_{C-F}=253.5 \mathrm{~Hz}\right), 86.7,80.6,73.3,70.7,63.4,63.2,20.9$, 20.7, 20.6, 14.0. HRMS Calcd for $\mathrm{C}_{26} \mathrm{H}_{26} \mathrm{~F}_{2} \mathrm{~N}_{4} \mathrm{O}_{9}\left[\mathrm{M}+\mathrm{H}^{+}\right]$: 577.1741, Found: 577.1749.

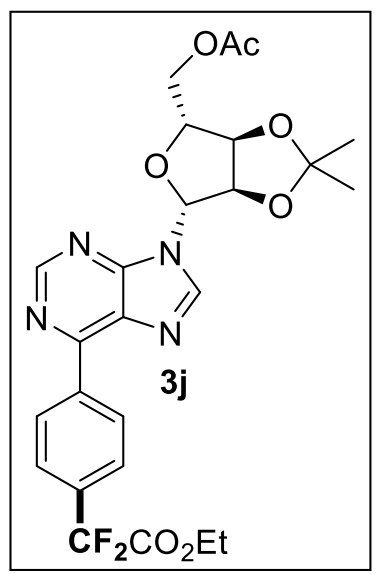

Yellow oil. Isolated yield: $69.2 \mathrm{mg}, 65 \%$.

${ }^{1} \mathrm{H}$ NMR (400 MHz, Chloroform- $d$ ) $\delta 9.03(\mathrm{~s}, 1 \mathrm{H}), 8.84(\mathrm{~d}, J=8.0 \mathrm{~Hz}, 2 \mathrm{H}), 8.24(\mathrm{~s}, 1 \mathrm{H}), 7.78(\mathrm{~d}, J=8.0$ $\mathrm{Hz}, 2 \mathrm{H}), 6.22(\mathrm{~d}, J=4.0 \mathrm{~Hz}, 1 \mathrm{H}), 5.50(\mathrm{dd}, J=8.0,4.0 \mathrm{~Hz}, 1 \mathrm{H}), 5.07(\mathrm{dd}, J=8.0,4.0 \mathrm{~Hz}, 1 \mathrm{H}), 4.52(\mathrm{dt}$, $J=6.0,4.0 \mathrm{~Hz}, 1 \mathrm{H}), 4.37(\mathrm{dd}, J=12.0,4.0 \mathrm{~Hz}, 1 \mathrm{H}), 4.33-4.19(\mathrm{~m}, 3 \mathrm{H}), 1.97(\mathrm{~s}, 3 \mathrm{H}), 1.63(\mathrm{~s}, 3 \mathrm{H}), 1.40$ $(\mathrm{s}, 3 \mathrm{H}), 1.29$ (t, $J=8.0 \mathrm{~Hz}, 3 \mathrm{H}) .{ }^{19} \mathrm{~F}$ NMR $\left(376 \mathrm{MHz}\right.$, Chloroform- $d$ ) $\delta-104.35 .{ }^{13} \mathrm{C}$ NMR $(100 \mathrm{MHz}$, Chloroform- $d) \delta 170.5,164.0\left(\mathrm{t}, J_{C-F}=35.1 \mathrm{~Hz}\right), 153.9,152.6,151.9,143.9,138.1,135.1\left(\mathrm{t}, J_{C-F}=25.6\right.$ $\mathrm{Hz}), 132.1,130.1,125.9\left(\mathrm{t}, J_{C-F}=6.1 \mathrm{~Hz}\right), 114.9,113.3\left(\mathrm{t}, J_{C-F}=252.4 \mathrm{~Hz}\right), 91.3,85.0,84.3,81.64,77.5$, 


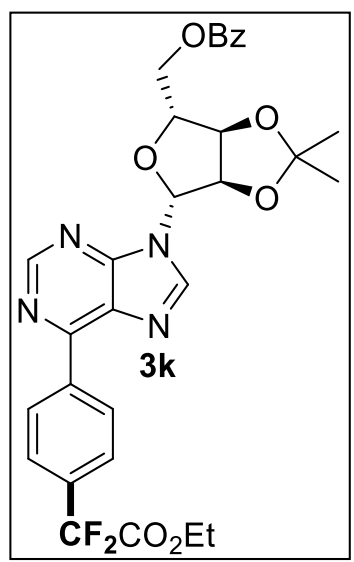

Yellow oil. Isolated yield: $74.8 \mathrm{mg}, 63 \%$.

${ }^{1} \mathrm{H}$ NMR $(400 \mathrm{MHz},) \delta 8.99(\mathrm{~s}, 1 \mathrm{H}), 8.78(\mathrm{~d}, J=8.0 \mathrm{~Hz}, 2 \mathrm{H}), 8.24(\mathrm{~s}, 1 \mathrm{H}), 7.85(\mathrm{~d}, J=8.0 \mathrm{~Hz}, 2 \mathrm{H}), 7.78$ $(\mathrm{d}, J=8.0 \mathrm{~Hz}, 2 \mathrm{H}), 7.46(\mathrm{t}, J=8.0 \mathrm{~Hz}, 1 \mathrm{H}), 7.30(\mathrm{t}, J=6.0 \mathrm{~Hz}, 2 \mathrm{H}), 6.22(\mathrm{~d}, J=2.0 \mathrm{~Hz}, 1 \mathrm{H}), 5.65(\mathrm{dd}$, $J=8.0,2.0 \mathrm{~Hz}, 1 \mathrm{H}), 5.20(\mathrm{dd}, J=8.0,4.0 \mathrm{~Hz}, 1 \mathrm{H}), 4.74-4.61(\mathrm{~m}, 2 \mathrm{H}), 4.54-4.45(\mathrm{~m}, 1 \mathrm{H}), 4.31(\mathrm{q}, J$ $=7.2 \mathrm{~Hz}, 2 \mathrm{H}), 1.66(\mathrm{~s}, 3 \mathrm{H}), 1.44(\mathrm{~s}, 3 \mathrm{H}), 1.30(\mathrm{t}, J=8.0 \mathrm{~Hz}, 3 \mathrm{H}) .{ }^{19} \mathrm{~F}$ NMR $(376 \mathrm{MHz}$, Chloroform- $d) \delta$ -104.33. ${ }^{13} \mathrm{C}$ NMR (100 MHz, Chloroform- $d$ ) $\delta 166.1,164.1\left(\mathrm{t}, J_{C-F}=35.2 \mathrm{~Hz}\right), 153.9,152.6,151.9$, $143.8,138.1,135.1\left(\mathrm{t}, J_{C-F}=25.6 \mathrm{~Hz}\right), 133.4,132.2,130.1,129.6,129.3,128.4,125.9\left(\mathrm{t}, J_{C-F}=6.1 \mathrm{~Hz}\right)$, 115.0, 113.4(t, $\left.J_{C-F}=253.5 \mathrm{~Hz}\right), 91.9,85.2,84.3,81.7,64.4,63.4,27.3,25.5,14.0$. HRMS Calcd for $\mathrm{C}_{30} \mathrm{H}_{28} \mathrm{~F}_{2} \mathrm{~N}_{4} \mathrm{O}_{7}\left[\mathrm{M}+\mathrm{H}^{+}\right]$: 595.1999, Found: 595.1993.

\section{Procedures for preparation 5 or 13.}
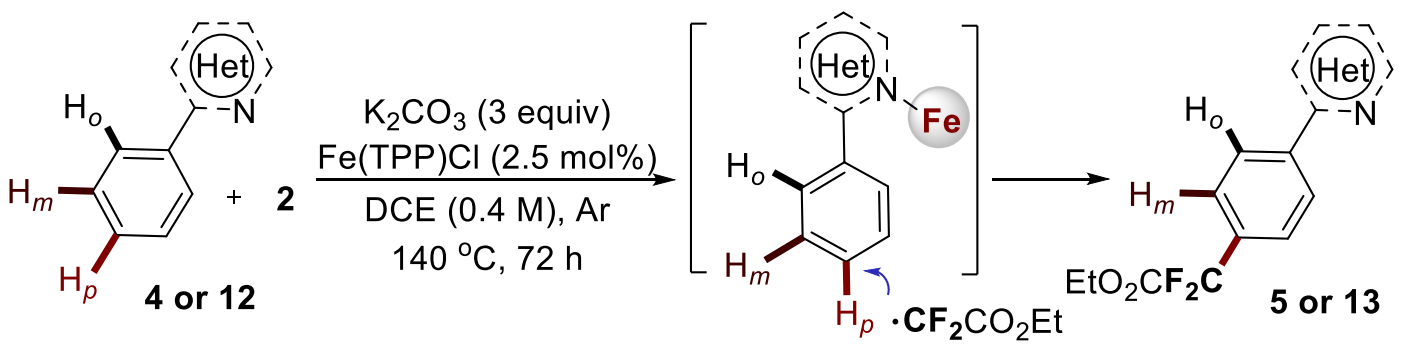

A mixture of 4 or 12 (0.2 mmol, 1.0 equiv), 2 (1.2 mmol, $154 \mu \mathrm{L}, 242.4 \mathrm{mg}, 6$ equiv), Fe(Tpp)Cl (3.5 mg, $2.5 \mathrm{~mol} \%$ ), $\mathrm{K}_{2} \mathrm{CO}_{3}(81.6 \mathrm{mg}, 300 \mathrm{~mol} \%$ ), and DCE $(0.5 \mathrm{~mL})$ in a $15 \mathrm{~mL}$ glass vial sealed under argon atmosphere was heated at $140{ }^{\circ} \mathrm{C}$ for 72 hours. The reaction mixture was cooled to room temperature and concentrated in vacuo. The resulting residue was purified by column chromatography $(\mathrm{PE} / \mathrm{EA}=30: 1)$ on silica gel to give the product $\mathbf{5}$ or $\mathbf{1 3}$. 


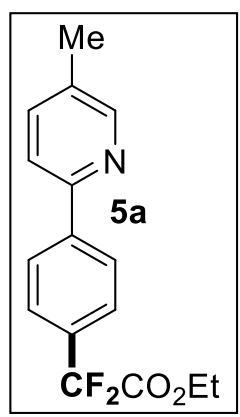

White solid. Isolated yield: $51.2 \mathrm{mg}, 88 \%$.

${ }^{1} \mathrm{H}$ NMR (400 MHz, Chloroform- $d$ ) $\delta 8.53$ (s, 1H), 8.05 (d, $\left.J=8.4 \mathrm{~Hz}, 2 \mathrm{H}\right), 7.69$ (d, $J=8.4 \mathrm{~Hz}, 2 \mathrm{H}$ ), $7.64(\mathrm{~d}, J=8.0 \mathrm{~Hz}, 1 \mathrm{H}), 7.57(\mathrm{~d}, J=8.0 \mathrm{~Hz}, 1 \mathrm{H}), 4.30(\mathrm{q}, J=8.0 \mathrm{~Hz}, 2 \mathrm{H}), 2.38(\mathrm{~s}, 3 \mathrm{H}), 1.30(\mathrm{t}, J=4.0$ $\mathrm{Hz}, 3 \mathrm{H}) .{ }^{19} \mathrm{~F}$ NMR $\left(376 \mathrm{MHz}\right.$, Chloroform- $d$ ) $\delta$-103.96. ${ }^{13} \mathrm{C}$ NMR $(100 \mathrm{MHz}$, Chloroform- $d$ ) $\delta 164.3(\mathrm{t}$, $\left.J_{C-F}=35.4 \mathrm{~Hz}\right), 153.6,150.4,142.1,137.6,132.9\left(\mathrm{t}, J_{C-F}=26.3 \mathrm{~Hz}\right), 132.6,127.0,126.0\left(\mathrm{t}, J_{C-F}=6.1\right.$ $\mathrm{Hz}), 120.4,113.6\left(\mathrm{t}, J_{C-F}=252.1 \mathrm{~Hz}\right), 63.3,18.3,14.0$. HRMS Calcd for $\mathrm{C}_{16} \mathrm{H}_{15} \mathrm{~F}_{2} \mathrm{NO}_{2}\left[\mathrm{M}+\mathrm{H}^{+}\right]: 292.1144$, Found: 292.1140.<smiles>CCOC(=O)c1cc(-c2ccc(C)cn2)ccc1C(F)(F)F</smiles>

White solid. Isolated yield: $47.6 \mathrm{mg} 78 \%$.

${ }^{1} \mathrm{H}$ NMR (400 MHz, Chloroform- $d$ ) $\delta 8.52(\mathrm{~s}, 1 \mathrm{H}), 7.87(\mathrm{~s}, 1 \mathrm{H}), 7.82(\mathrm{~d}, J=8.0 \mathrm{~Hz}, 1 \mathrm{H}), 7.69-7.63(\mathrm{~m}$, 2H), 7.57 (d, $J=8.0 \mathrm{~Hz}, 1 \mathrm{H}), 4.32(\mathrm{q}, J=7.2 \mathrm{~Hz}, 2 \mathrm{H}), 2.50(\mathrm{~s}, 3 \mathrm{H}), 2.38(\mathrm{~s}, 3 \mathrm{H}), 1.30$ (t, $J=7.2 \mathrm{~Hz}$, $3 \mathrm{H}) .{ }^{19} \mathrm{~F}$ NMR $\left(376 \mathrm{MHz}\right.$, Chloroform- $d$ ) $\delta-101.35 .{ }^{13} \mathrm{C}$ NMR $\left(100 \mathrm{MHz}\right.$, Chloroform- $d$ ) $\delta 164.3\left(\mathrm{t}, J_{C \text { - }}\right.$ $\left.{ }_{F}=35.2 \mathrm{~Hz}\right), 153.7,150.4,141.7,137.5,137.0,132.5,131.3\left(\mathrm{t}, J_{C-F}=23.4 \mathrm{~Hz}\right), 130.2,126.8\left(\mathrm{t}, J_{C-F}=\right.$ $8.8 \mathrm{~Hz}), 124.2,120.5,114.4\left(\mathrm{t}, J_{C-F}=251.7 \mathrm{~Hz}\right), 63.3,20.0,18.4,14.0$. HRMS Calcd for $\mathrm{C}_{17} \mathrm{H}_{17} \mathrm{~F}_{2} \mathrm{NO}_{2}$ $\left[\mathrm{M}+\mathrm{H}^{+}\right]$: 306.1300, Found: 306.1303.

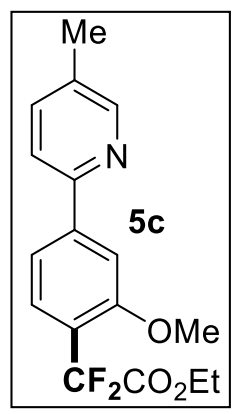

White solid. Isolated yield: $53.9 \mathrm{mg} 84 \%$.

${ }^{1} \mathrm{H}$ NMR (400 MHz, Chloroform- $d$ ) $\delta 8.52(\mathrm{~s}, 1 \mathrm{H}), 7.71(\mathrm{~d}, J=8.0 \mathrm{~Hz}, 1 \mathrm{H}), 7.68(\mathrm{~s}, 1 \mathrm{H}), 7.65(\mathrm{~d}, J=8.0$ $\mathrm{Hz}, 1 \mathrm{H}), 7.58(\mathrm{dd}, J=8.0,2.0 \mathrm{~Hz}, 1 \mathrm{H}), 7.55(\mathrm{dd}, J=8.0,1.4 \mathrm{~Hz}, 1 \mathrm{H}), 4.33$ (q, $J=7.2 \mathrm{~Hz}, 2 \mathrm{H}), 3.92$ (s, $3 \mathrm{H}), 2.39(\mathrm{~s}, 3 \mathrm{H}), 1.29(\mathrm{t}, J=7.2 \mathrm{~Hz}, 3 \mathrm{H}) .{ }^{19} \mathrm{~F}$ NMR $\left(376 \mathrm{MHz}\right.$, Chloroform- $d$ ) $\delta-102.63 .{ }^{13} \mathrm{C}$ NMR $(100$ $\mathrm{MHz}$, Chloroform- $d) \delta 164.15\left(\mathrm{t}, J_{C-F}=34.1 \mathrm{~Hz}\right), 157.3,153.6,150.3,143.8,137.6,132.7,126.8\left(\mathrm{t}, J_{C \text { - }}\right.$ 
$\left.F_{F}=7.4 \mathrm{~Hz}\right), 122.2\left(\mathrm{t}, J_{C-F}=24.1 \mathrm{~Hz}\right), 120.5,118.8,112.4\left(\mathrm{t}, J_{C-F}=248.46 \mathrm{~Hz}\right), 109.8,62.8,56.0,18.4$, 14.1. HRMS Calcd for $\mathrm{C}_{17} \mathrm{H}_{17} \mathrm{~F}_{2} \mathrm{NO}_{3}\left[\mathrm{M}+\mathrm{Na}^{+}\right]$: 344.1069, Found: 344.1061 .

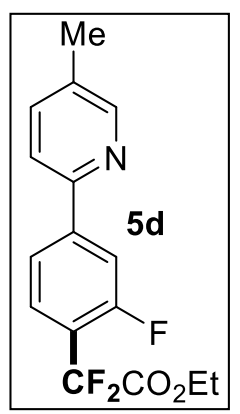

White solid. Isolated yield: $38.9 .4 \mathrm{mg}, 63 \%$.

${ }^{1} \mathrm{H}$ NMR (400 MHz, Chloroform- $d$ ) $\delta 8.53(\mathrm{~s}, 1 \mathrm{H}), 7.82(\mathrm{t}, J=8.0 \mathrm{~Hz}, 2 \mathrm{H}), 7.71(\mathrm{t}, J=4.0 \mathrm{~Hz}, 1 \mathrm{H}), 7.65$ $-7.57(\mathrm{~m}, 2 \mathrm{H}), 4.36(\mathrm{q}, J=7.2 \mathrm{~Hz}, 2 \mathrm{H}), 2.39(\mathrm{~s}, 3 \mathrm{H}), 1.31(\mathrm{t}, J=7.2 \mathrm{~Hz}, 3 \mathrm{H}) .{ }^{19} \mathrm{~F}$ NMR $(376 \mathrm{MHz}$, Chloroform- $d$ ) $\delta-101.98$ (d, $J=8.0 \mathrm{~Hz}),-113.65--116.06$ (m). ${ }^{13} \mathrm{C}$ NMR $(100 \mathrm{MHz}$, Chloroform- $d$ ) $\delta$ $163.3\left(\mathrm{t}, J_{C-F}=34.1 \mathrm{~Hz}\right), 160.3\left(\mathrm{dt}, J_{C-F}=251.9,4.8 \mathrm{~Hz}\right), 152.2\left(\mathrm{~d}, J_{C-F}=2.2 \mathrm{~Hz}\right), 150.5,144.7\left(\mathrm{~d}, J_{C-F}\right.$ $=8.0 \mathrm{~Hz}), 137.7,133.3,127.5\left(\mathrm{td}, J_{C-F}=6.9,2.5 \mathrm{~Hz}\right), 122.2\left(\mathrm{~d}, J_{C-F}=3.2 \mathrm{~Hz}\right), 120.8\left(\mathrm{td}, J_{C-F}=25.9\right.$, $13.0 \mathrm{~Hz}), 120.4,114.6\left(\mathrm{~d}, J_{C-F}=22.3 \mathrm{~Hz}\right), 111.8\left(\mathrm{t}, J_{C-F}=251.1 \mathrm{~Hz}\right), 63.5,18.4$, 14.0. HRMS Calcd for $\mathrm{C}_{16} \mathrm{H}_{14} \mathrm{~F}_{3} \mathrm{NO}_{2}\left[\mathrm{M}+\mathrm{H}^{+}\right]$: 310.1049, Found: 310.1050 .

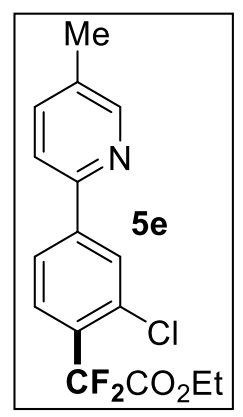

White solid. Isolated yield: $22.1 \mathrm{mg}, 34 \%$.

${ }^{1} \mathrm{H}$ NMR (400 MHz, Chloroform- $d$ ) $\delta 8.54(\mathrm{~s}, 1 \mathrm{H}), 8.10(\mathrm{~s}, 1 \mathrm{H}), 7.95(\mathrm{~d}, J=12.0 \mathrm{~Hz}, 1 \mathrm{H}), 7.81(\mathrm{~d}, J=$ $8.0 \mathrm{~Hz}, 1 \mathrm{H}), 7.65(\mathrm{~d}, J=8.0 \mathrm{~Hz}, 1 \mathrm{H}), 7.62-7.56(\mathrm{~m}, 1 \mathrm{H}), 4.36(\mathrm{q}, J=7.2 \mathrm{~Hz}, 2 \mathrm{H}), 2.40(\mathrm{~s}, 3 \mathrm{H}), 1.31(\mathrm{t}$, $J=7.2 \mathrm{~Hz}, 3 \mathrm{H}) .{ }^{19} \mathrm{~F}$ NMR $\left(376 \mathrm{MHz}\right.$, Chloroform- $d$ ) $\delta-102.21 .{ }^{13} \mathrm{C}$ NMR $(100 \mathrm{MHz}$, Chloroform- $d$ ) $\delta$ $163.3\left(\mathrm{t}, J_{C-F}=34.0 \mathrm{~Hz}\right), 152.2,150.6,143.5,137.7,133.3,132.6\left(\mathrm{t}, J_{C-F}=4.3 \mathrm{~Hz}\right), 131.1\left(\mathrm{t}, J_{C-F}=24.4\right.$ $\mathrm{Hz}), 128.9,127.7,124.9,120.4,112.4\left(\mathrm{t}, J_{C-F}=250.7 \mathrm{~Hz}\right), 63.5,18.4$, 14.0. HRMS Calcd for $\mathrm{C}_{16} \mathrm{H}_{14} \mathrm{ClF}_{2} \mathrm{NO}_{2}\left[\mathrm{M}+\mathrm{H}^{+}\right]: 326.0754$, Found: 326.0752 .

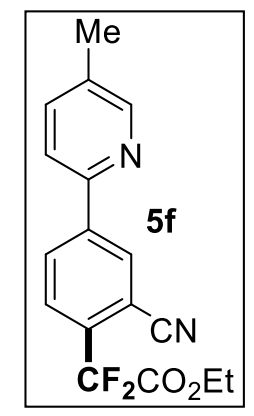

White solid. Isolated yield: $24.0 \mathrm{mg}, 38 \%$.

${ }^{1} \mathrm{H}$ NMR (400 MHz, Chloroform- $d$ ) $\delta 8.55(\mathrm{~s}, 1 \mathrm{H}), 8.45(\mathrm{~s}, 1 \mathrm{H}), 8.28(\mathrm{~d}, J=12.0 \mathrm{~Hz}, 1 \mathrm{H}), 7.83(\mathrm{~d}, J=$ $8.0 \mathrm{~Hz}, 1 \mathrm{H}), 7.68(\mathrm{~d}, J=12.0 \mathrm{~Hz}, 1 \mathrm{H}), 7.63(\mathrm{dd}, J=8.0,1.6 \mathrm{~Hz}, 1 \mathrm{H}), 4.38(\mathrm{q}, J=7.2 \mathrm{~Hz}, 2 \mathrm{H}), 2.41(\mathrm{~s}$, 
$3 \mathrm{H}), 1.35(\mathrm{t}, J=7.2 \mathrm{~Hz}, 3 \mathrm{H}) .{ }^{19} \mathrm{~F}$ NMR $\left(376 \mathrm{MHz}\right.$, Chloroform- $d$ ) $\delta-101.43 .{ }^{13} \mathrm{C}$ NMR $(100 \mathrm{MHz}$, Chloroform- $d) \delta 162.7\left(\mathrm{t}, J_{C-F}=34.3 \mathrm{~Hz}\right), 151.1,150.8,142.6,137.9,135.0\left(\mathrm{t}, J_{C-F}=25.2 \mathrm{~Hz}\right), 133.9$, $132.9,130.6,127.6\left(\mathrm{t}, J_{C-F}=7.3 \mathrm{~Hz}\right), 120.3,116.2,112.4\left(\mathrm{t}, J_{C-F}=254.4 \mathrm{~Hz}\right), 111.1\left(\mathrm{t}, J_{C-F}=3.8 \mathrm{~Hz}\right)$, 64.1, 18.4, 14.0. HRMS Calcd for $\mathrm{C}_{17} \mathrm{H}_{14} \mathrm{~F}_{2} \mathrm{~N}_{2} \mathrm{O}_{2}\left[\mathrm{M}+\mathrm{H}^{+}\right]$: 317.1096, Found: 317.1098 .<smiles>CCOC(=O)c1ccc(-c2ccc(CCc3ccc(C(F)(F)F)cc3)cn2)cc1</smiles>

White solid. Isolated yield: $51.3 \mathrm{mg}, 85 \%$.

${ }^{1} \mathrm{H}$ NMR (400 MHz, Chloroform- $d$ ) $\delta 8.96(\mathrm{~s}, 1 \mathrm{H}), 8.13(\mathrm{~d}, J=12.0 \mathrm{~Hz}, 2 \mathrm{H}), 8.05(\mathrm{dd}, J=12.0,2.1 \mathrm{~Hz}$, $1 \mathrm{H}), 7.88(\mathrm{~d}, J=8.0 \mathrm{~Hz}, 1 \mathrm{H}), 7.76(\mathrm{~d}, J=8.0 \mathrm{~Hz}, 2 \mathrm{H}), 4.32(\mathrm{q}, J=7.2 \mathrm{~Hz}, 2 \mathrm{H}), 1.31(\mathrm{t}, J=7.2 \mathrm{~Hz}, 3 \mathrm{H})$. ${ }^{19} \mathrm{~F}$ NMR (376 MHz, Chloroform- $d$ ) $\delta-104.22 .{ }^{13} \mathrm{C}$ NMR (100 MHz, Chloroform- $d$ ) $\delta 164.0\left(\mathrm{t}, J_{C-F}=\right.$ $35.0 \mathrm{~Hz}), 159.3,152.7,140.3,140.0,134.9\left(\mathrm{t}, J_{C-F}=25.7 \mathrm{~Hz}\right), 127.8,126.4\left(\mathrm{t}, J_{C-F}=6.1 \mathrm{~Hz}\right), 120.5$, 116.8, $113.3\left(\mathrm{t}, J_{C-F}=252.6 \mathrm{~Hz}\right), 108.8,63.5,14.0$. HRMS Calcd for $\mathrm{C}_{16} \mathrm{H}_{12} \mathrm{~F}_{2} \mathrm{~N}_{2} \mathrm{O}_{2}\left[\mathrm{M}+\mathrm{H}^{+}\right]$: 303.0940, Found: 303.0946.<smiles>CCOC(=O)Cc1cccc(-c2ccc(C(F)(F)F)cc2)n1</smiles>

White solid. Isolated yield: $39.8 \mathrm{mg}, 64 \%$.

${ }^{1} \mathrm{H}$ NMR (400 MHz, Chloroform- $d$ ) $\delta 8.08(\mathrm{~d}, J=8.0 \mathrm{~Hz}, 2 \mathrm{H}), 7.77-7.61(\mathrm{~m}, 4 \mathrm{H}), 7.31(\mathrm{~d}, J=8.0 \mathrm{~Hz}$, $1 \mathrm{H}), 4.31(\mathrm{q}, J=7.2 \mathrm{~Hz}, 2 \mathrm{H}), 1.30(\mathrm{t}, J=7.2 \mathrm{~Hz}, 3 \mathrm{H}) .{ }^{19} \mathrm{~F}$ NMR $(376 \mathrm{MHz}$, Chloroform- $d$ ) $\delta-104.16$. ${ }^{13} \mathrm{C}$ NMR $\left(100 \mathrm{MHz}\right.$, Chloroform- $d$ ) $\delta 164.1\left(\mathrm{t}, J_{C-F}=35.2 \mathrm{~Hz}\right), 156.9,151.8,140.4,139.6,133.9\left(\mathrm{t}, J_{C-}\right.$ $\left.F_{F}=25.7 \mathrm{~Hz}\right), 127.4,126.1\left(\mathrm{t}, J_{C-F}=6.1 \mathrm{~Hz}\right), 123.5,119.1,113.4\left(\mathrm{t}, J_{C-F}=252.3 \mathrm{~Hz}\right), 63.4,14.0$. HRMS Calcd for $\mathrm{C}_{15} \mathrm{H}_{12} \mathrm{ClF}_{2} \mathrm{NO}_{2}\left[\mathrm{M}+\mathrm{H}^{+}\right]: 312.0597$, Found: 312.0601 .

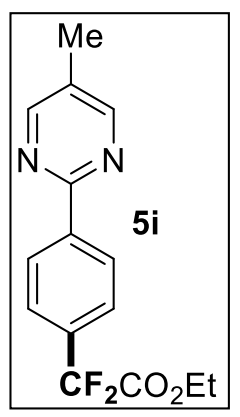

White solid. Isolated yield: $52.6 \mathrm{mg}, 90 \%$.

${ }^{1} \mathrm{H}$ NMR (400 MHz, Chloroform- $d$ ) $\delta 8.64(\mathrm{~s}, 2 \mathrm{H}), 8.49(\mathrm{~d}, J=8.0 \mathrm{~Hz}, 2 \mathrm{H}), 7.71(\mathrm{~d}, J=8.0 \mathrm{~Hz}, 2 \mathrm{H})$, $4.30(\mathrm{q}, J=7.2 \mathrm{~Hz}, 3 \mathrm{H}), 2.35$ (s, 3H), 1.29 (t, $J=7.2 \mathrm{~Hz}, 3 \mathrm{H}) .{ }^{19} \mathrm{~F}$ NMR $(376 \mathrm{MHz}$, Chloroform- $d$ ) $\delta$ 104.11 ${ }^{13} \mathrm{C}$ NMR (100 MHz, Chloroform- $\left.d\right) \delta 164.2\left(\mathrm{t}, J_{C-F}=35.3 \mathrm{~Hz}\right), 161.5,157.6,140.3,134.5(\mathrm{t}$, 
$\left.J_{C-F}=25.6 \mathrm{~Hz}\right), 129.2,128.2,125.9\left(\mathrm{t}, J_{C-F}=6.0 \mathrm{~Hz}\right), 113.5\left(\mathrm{t}, J_{C-F}=252.2 \mathrm{~Hz}\right), 63.3,15.7,14.0$. HRMS Calcd for $\mathrm{C}_{15} \mathrm{H}_{14} \mathrm{~F}_{2} \mathrm{~N}_{2} \mathrm{O}_{2}\left[\mathrm{M}+\mathrm{H}^{+}\right]$: 293.1096, Found: 293.1099.<smiles></smiles>

White solid. Isolated yield: $53.2 \mathrm{mg}, 87 \%$.

${ }^{1} \mathrm{H}$ NMR (400 MHz, Chloroform- $d$ ) $\delta 8.63(\mathrm{~s}, 2 \mathrm{H}), 8.28(\mathrm{~d}, J=8.0 \mathrm{~Hz}, 2 \mathrm{H}), 7.68(\mathrm{~d}, J=12.0 \mathrm{~Hz}, 1 \mathrm{H})$, $4.31(\mathrm{q}, J=7.2 \mathrm{~Hz}, 2 \mathrm{H}), 2.50$ (s, 3H), 2.34 (s, 3H), 1.28 (t, $J=7.2 \mathrm{~Hz}, 3 \mathrm{H}) .{ }^{19} \mathrm{~F}$ NMR $(376 \mathrm{MHz}$, Chloroform- $d$ ) $\delta-101.50 .{ }^{13} \mathrm{C}$ NMR $\left(100 \mathrm{MHz}\right.$, Chloroform- $d$ ) $\delta 164.2\left(\mathrm{t}, J_{C-F}=35.0 \mathrm{~Hz}\right), 161.6,157.6$, $139.9,136.9\left(\mathrm{t}, J_{C-F}=3.0 \mathrm{~Hz}\right), 132.9\left(\mathrm{t}, J_{C-F}=23.4 \mathrm{~Hz}\right), 131.2,129.1,126.7\left(\mathrm{t}, J_{C-F}=8.8 \mathrm{~Hz}\right), 125.5$, $114.3\left(\mathrm{t}, J_{C-F}=251.9 \mathrm{~Hz}\right.$ ), 63.3, 19.9, 15.6, 14.0. HRMS Calcd for $\mathrm{C}_{16} \mathrm{H}_{16} \mathrm{~F}_{2} \mathrm{~N}_{2} \mathrm{O}_{2}\left[\mathrm{M}+\mathrm{H}^{+}\right]$: 307.1253, Found: 307.1257.<smiles>CCOc1cc(CCc2cnc(-c3ccc(C(F)F)c(OC)c3)nc2)ccc1CC</smiles>

White solid. Isolated yield: $56.0 \mathrm{mg}, 87 \%$.

${ }^{1} \mathrm{H}$ NMR (400 MHz, Chloroform- $d$ ) $\delta 8.62(\mathrm{~s}, 2 \mathrm{H}), 8.11(\mathrm{~d}, J=8.1 \mathrm{~Hz}, 1 \mathrm{H}), 8.02(\mathrm{~s}, 1 \mathrm{H}), 7.73(\mathrm{~d}, J=8.1$ $\mathrm{Hz}, 1 \mathrm{H}), 4.32(\mathrm{q}, J=7.1 \mathrm{~Hz}, 2 \mathrm{H}), 3.92(\mathrm{~s}, 3 \mathrm{H}), 2.33(\mathrm{~s}, 3 \mathrm{H}), 1.28(\mathrm{t}, J=7.1 \mathrm{~Hz}, 3 \mathrm{H}) .{ }^{19} \mathrm{~F}$ NMR $(376 \mathrm{MHz}$, Chloroform- $d$ ) $\delta-102.78 .{ }^{13} \mathrm{C}$ NMR $\left(100 \mathrm{MHz}\right.$, Chloroform- $d$ ) $\delta 164.0\left(\mathrm{t}, J_{C-F}=33.9 \mathrm{~Hz}\right), 161.3,157.5$, $157.1\left(\mathrm{t}, J_{C-F}=5.0 \mathrm{~Hz}\right), 141.8,129.3,126.7\left(\mathrm{t}, J_{C-F}=7.4 \mathrm{~Hz}\right), 123.7\left(\mathrm{t}, J_{C-F}=24.0 \mathrm{~Hz}\right), 120.3,112.3(\mathrm{t}$, $\left.J_{C-F}=249.47 \mathrm{~Hz}\right), 110.6,62.8,56.0,15.6,14.0$. HRMS Calcd for $\mathrm{C}_{16} \mathrm{H}_{16} \mathrm{~F}_{2} \mathrm{~N}_{2} \mathrm{O}_{3}\left[\mathrm{M}+\mathrm{H}^{+}\right]$: 323.1202, Found: 323.1209 .<smiles></smiles>

White solid. Isolated yield: $39.1 \mathrm{mg}, 63 \%$.

${ }^{1} \mathrm{H}$ NMR (400 MHz, Chloroform- $d$ ) $\delta 8.65(\mathrm{~s}, 2 \mathrm{H}), 8.31(\mathrm{~d}, J=8.0 \mathrm{~Hz}, 1 \mathrm{H}), 8.20(\mathrm{~d}, J=12.0 \mathrm{~Hz}, 1 \mathrm{H})$, $7.74(\mathrm{t}, J=8.0 \mathrm{~Hz}, 1 \mathrm{H}), 4.36$ (q, $J=7.2 \mathrm{~Hz}, 2 \mathrm{H}), 2.37$ (s, 3H), 1.32 (t, $J=7.2 \mathrm{~Hz}, 3 \mathrm{H}) .{ }^{19} \mathrm{~F}$ NMR $(376$ MHz, Chloroform- $d$ ) $\delta-102.12(\mathrm{~d}, J=7.1 \mathrm{~Hz}),-114.47$ (t, $J=7.5 \mathrm{~Hz}) \cdot{ }^{13} \mathrm{C}$ NMR $(100 \mathrm{MHz}$, Chloroform- 
d) $\delta 163.3\left(\mathrm{t}, J_{C-F}=34.0 \mathrm{~Hz}\right), 160.3\left(\mathrm{~d}, J_{C-F}=2.9 \mathrm{~Hz}\right), 161.7-158.5(\mathrm{~m}), 157.7,143.0\left(\mathrm{~d}, J_{C-F}=8.3 \mathrm{~Hz}\right)$, $129.8,127.4\left(\mathrm{td}, J_{C-F}=6.9,2.2 \mathrm{~Hz}\right), 123.6\left(\mathrm{~d}, J_{C-F}=3.3 \mathrm{~Hz}\right), 123.0-121.8(\mathrm{~m}), 115.7\left(\mathrm{~d}, J_{C-F}=22.8\right.$ $\mathrm{Hz}), 111.8\left(\mathrm{t}, J_{C-F}=251.2 \mathrm{~Hz}\right), 63.5,15.7,15.0$. HRMS Calcd for $\mathrm{C}_{15} \mathrm{H}_{13} \mathrm{~F}_{3} \mathrm{~N}_{2} \mathrm{O}_{2}\left[\mathrm{M}+\mathrm{H}^{+}\right]$: 311.1002, Found: 311.1010 .

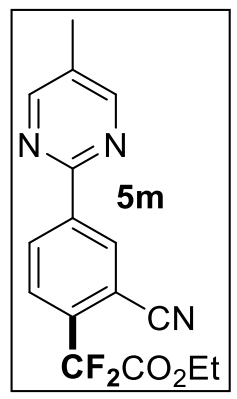

White solid. Isolated yield: $26.0 \mathrm{mg}, 41 \%$.

${ }^{1} \mathrm{H}$ NMR (400 MHz, Chloroform- $d$ ) $\delta 8.89(\mathrm{~s}, 1 \mathrm{H}), 8.74(\mathrm{~d}, J=8.0 \mathrm{~Hz}, 1 \mathrm{H}), 8.68(\mathrm{~s}, 2 \mathrm{H}), 7.86(\mathrm{~d}, J=8.0$ $\mathrm{Hz}, 1 \mathrm{H}$ ), 4.39 (q, $J=7.2 \mathrm{~Hz}, 2 \mathrm{H}$ ), 2.39 (s, 3H), 1.36 (t, $J=7.2 \mathrm{~Hz}, 3 \mathrm{H}) .{ }^{19} \mathrm{~F}$ NMR (376 MHz, Chloroformd) $\delta$-101.53. ${ }^{13} \mathrm{C}$ NMR (100 MHz, Chloroform- $d$ ) $\delta 162.7\left(\mathrm{t}, J_{C-F}=34.1 \mathrm{~Hz}\right), 159.4,157.8,141.0,136.5$ $\left(\mathrm{t}, J_{C-F}=25.0 \mathrm{~Hz}\right), 134.2,131.8,130.3,127.5\left(\mathrm{t}, J_{C-F}=7.4 \mathrm{~Hz}\right), 116.2,112.4\left(\mathrm{t}, J_{C-F}=255.5 \mathrm{~Hz}\right), 111.0$ $\left(\mathrm{t}, J_{C-F}=3.8 \mathrm{~Hz}\right), 64.1,15.8,14.0$. HRMS Calcd for $\mathrm{C}_{16} \mathrm{H}_{13} \mathrm{~F}_{2} \mathrm{~N}_{3} \mathrm{O}_{2}\left[\mathrm{M}+\mathrm{H}^{+}\right]$: 318.1049, Found: 318.1056.<smiles>CCOC(=O)c1ccc(-c2ncc(Br)cc2CBr)cc1</smiles>

White solid. Isolated yield: $42.7 \mathrm{mg}, 60 \%$.

${ }^{1} \mathrm{H}$ NMR (400 MHz, Chloroform- $d$ ) $\delta 8.85(\mathrm{~s}, 2 \mathrm{H}), 8.49(\mathrm{~d}, J=8.0 \mathrm{~Hz}, 2 \mathrm{H}), 7.72(\mathrm{~d}, J=12.0 \mathrm{~Hz}, 2 \mathrm{H})$, 4.31 (q, $J=7.2 \mathrm{~Hz}, 3 \mathrm{H}), 1.30$ (t, $J=7.2 \mathrm{~Hz}, 3 \mathrm{H}) .{ }^{19} \mathrm{~F}$ NMR $\left(376 \mathrm{MHz}\right.$, Chloroform- $d$ ) $\delta-104.23 .{ }^{13} \mathrm{C}$ NMR (100 MHz, Chloroform- $d$ ) $\delta 164.1\left(\mathrm{t}, J_{C-F}=35.1 \mathrm{~Hz}\right), 161.9,158.1,139.2,135.3\left(\mathrm{t}, J_{C-F}=25.5\right.$ $\mathrm{Hz}), 128.5,126.0\left(\mathrm{t}, J_{C-F}=6.1 \mathrm{~Hz}\right), 119.2,113.4\left(\mathrm{t}, J_{C-F}=252.4 \mathrm{~Hz}\right), 63.4,14.0$. HRMS Calcd for $\mathrm{C}_{14} \mathrm{H}_{11} \mathrm{BrF}_{2} \mathrm{~N}_{2} \mathrm{O}_{2}\left[\mathrm{M}+\mathrm{H}^{+}\right]: 357.0045$, Found: 357.0041 .<smiles>CCOC(=O)c1ccc(-c2nc(Cl)cc(Cl)n2)cc1</smiles>

White solid. Isolated yield: $53.3 \mathrm{mg}, 77 \%$.

${ }^{1} \mathrm{H}$ NMR (400 MHz, Chloroform- $d$ ) $\delta 8.51(\mathrm{~d}, J=8.0 \mathrm{~Hz}, 2 \mathrm{H}), 7.73(\mathrm{~d}, J=8.0 \mathrm{~Hz}, 2 \mathrm{H}), 7.31(\mathrm{~s}, 1 \mathrm{H})$, $4.31(\mathrm{q}, J=7.2 \mathrm{~Hz}, 2), 1.30(\mathrm{t}, J=7.2 \mathrm{~Hz}, 3 \mathrm{H}) .{ }^{19} \mathrm{~F}$ NMR $\left(376 \mathrm{MHz}\right.$, Chloroform- $d$ ) $\delta-104.37 .{ }^{13} \mathrm{C}$ NMR $\left(100 \mathrm{MHz}\right.$, Chloroform- $d$ ) $\delta 164.6,163.9\left(\mathrm{t}, J_{C-F}=34.9 \mathrm{~Hz}\right), 162.4,137.5,136.3\left(\mathrm{t}, J_{C-F}=25.6 \mathrm{~Hz}\right), 129.2$, 
$126.1\left(\mathrm{t}, J_{C-F}=6.1 \mathrm{~Hz}\right), 119.7,113.2\left(\mathrm{t}, J_{C-F}=252.6 \mathrm{~Hz}\right), 63.5,14.0$. HRMS Calcd for $\mathrm{C}_{14} \mathrm{H}_{10} \mathrm{Cl}_{2} \mathrm{~F}_{2} \mathrm{~N}_{2} \mathrm{O}_{2}$ [M+H'] 347.0160 , Found: 347.0156 .

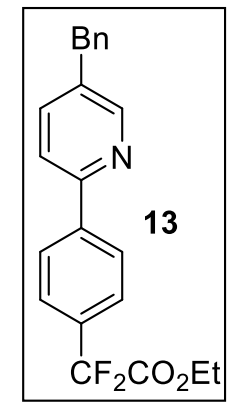

White solid. Isolated yield: $38.2 \mathrm{mg}, 52 \%$.

${ }^{1} \mathrm{H}$ NMR $(400 \mathrm{MHz}$, Chloroform- $d$ ) $\delta 8.60(\mathrm{~d}, J=4.0 \mathrm{~Hz}, 1 \mathrm{H}), 8.05(\mathrm{~d}, J=8.0 \mathrm{~Hz}, 2 \mathrm{H}), 7.70(\mathrm{~d}, J=8.0$ $\mathrm{Hz}, 2 \mathrm{H}), 7.66(\mathrm{~d}, J=8.0 \mathrm{~Hz}, 1 \mathrm{H}), 7.56(\mathrm{dd}, J=8.0,4.0 \mathrm{~Hz}, 1 \mathrm{H}), 7.35-7.28(\mathrm{~m}, 2 \mathrm{H}), 7.25-7.18(\mathrm{~m}$, $3 \mathrm{H}), 4.30$ (q, $J=7.2 \mathrm{~Hz}, 2 \mathrm{H}), 4.03$ (s, 2H), 1.30 (t, $J=7.2 \mathrm{~Hz}, 3 \mathrm{H}) .{ }^{19} \mathrm{~F}$ NMR (376 MHz, Chloroform- $d$ ) $\delta$-103.95. ${ }^{13} \mathrm{C}$ NMR $\left(100 \mathrm{MHz}\right.$, Chloroform- $d$ ) $\delta 164.3\left(\mathrm{t}, J_{C-F}=35.4 \mathrm{~Hz}\right), 154.2,150.3,141.9,139.8$, 137.4, 136.0, $133.1\left(\mathrm{t}, J_{C-F}=25.7 \mathrm{~Hz}\right), 129.0,128.9,127.1,126.7,126.1\left(\mathrm{t}, J_{C-F}=6.1 \mathrm{~Hz}\right), 120.7,113.5$ $\left(\mathrm{t}, J_{C-F}=252.1 \mathrm{~Hz}\right), 63.3,38.9,14.0$. HRMS Calcd for $\mathrm{C}_{22} \mathrm{H}_{19} \mathrm{~F}_{2} \mathrm{NO}_{2}\left[\mathrm{M}+\mathrm{H}^{+}\right]$: 368.15, Found: 368.10 .

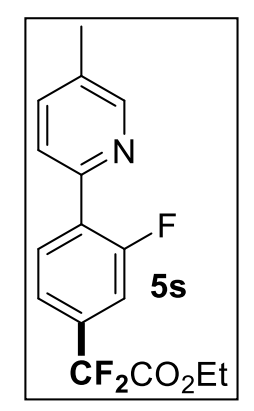

Yellow oil. Isolated yield: $19.8 \mathrm{mg}, 32 \%$.

${ }^{1} \mathrm{H}$ NMR (400 MHz, Chloroform- $d$ ) $\delta 8.56(\mathrm{~s}, 1 \mathrm{H}), 8.07(\mathrm{t}, J=8.0 \mathrm{~Hz}, 1 \mathrm{H}), 7.71(\mathrm{~d}, J=8.0 \mathrm{~Hz}, 1 \mathrm{H})$, 7.59 (d, $J=8.0 \mathrm{~Hz}, 1 \mathrm{H}), 7.49$ (d, $J=8.0 \mathrm{~Hz}, 1 \mathrm{H}), 7.42(\mathrm{~d}, J=8.0 \mathrm{~Hz}, 1 \mathrm{H}), 4.31$ (q, $J=7.2 \mathrm{~Hz}, 2 \mathrm{H}), 2.39$ $(\mathrm{s}, 3 \mathrm{H}), 1.31(\mathrm{t}, J=7.2 \mathrm{~Hz}, 3 \mathrm{H}) .{ }^{19} \mathrm{~F}$ NMR $\left(376 \mathrm{MHz}\right.$, Chloroform- $d$ ) $\delta-104.06,-115.49 .{ }^{13} \mathrm{C}$ NMR $(100$ MHz, Chloroform- $d$ ) $\delta 163.8\left(\mathrm{t}, J_{C-F}=34.9 \mathrm{~Hz}\right), 160.2\left(\mathrm{~d}, J_{C-F}=251.3 \mathrm{~Hz}\right), 150.6,149.5\left(\mathrm{~d}, J_{C-F}=2.7\right.$ $\mathrm{Hz}), 137.2,134.7\left(\mathrm{td}, J_{C-F}=26.5,8.0 \mathrm{~Hz}\right), 133.0,131.6\left(\mathrm{~d}, J_{C-F}=3.5 \mathrm{~Hz}\right), 130.2\left(\mathrm{~d}, J_{C-F}=11.7 \mathrm{~Hz}\right)$, $124.2\left(\mathrm{~d}, J_{C-F}=9.1 \mathrm{~Hz}\right), 121.7\left(\mathrm{td}, J_{C-F}=6.1,3.6 \mathrm{~Hz}\right), 114.0\left(\mathrm{dt}, J_{C-F}=26.4,6.5 \mathrm{~Hz}\right), 112.7,63.5,18.4$, 14.0. HRMS Calcd for $\mathrm{C}_{16} \mathrm{H}_{14} \mathrm{~F}_{3} \mathrm{NO}_{2}\left[\mathrm{M}+\mathrm{H}^{+}\right]$: 310.1049, Found: 310.1055 .

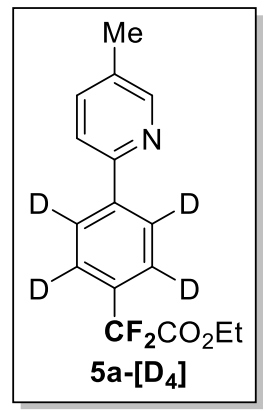

White solid. Isolated yield: $46.6 \mathrm{mg}, 79 \%$. 
${ }^{1} \mathrm{H}$ NMR (400 MHz, Chloroform- $d$ ) $\delta 8.53(\mathrm{~s}, 1 \mathrm{H}), 7.64(\mathrm{~d}, J=8.0 \mathrm{~Hz}, 1 \mathrm{H}), 7.58(\mathrm{dd}, J=8.0,4.0 \mathrm{~Hz}$, $1 \mathrm{H}), 4.30(\mathrm{q}, J=7.2 \mathrm{~Hz}, 2 \mathrm{H}), 2.38(\mathrm{~s}, 3 \mathrm{H}), 1.30(\mathrm{t}, J=7.2 \mathrm{~Hz}, 3 \mathrm{H})$. HRMS Calcd for $\mathrm{C}_{16} \mathrm{H}_{11} \mathrm{D}_{4} \mathrm{~F}_{2} \mathrm{NO}_{2}$ [M+H']: 296.1395, Found: 296.1398 .

\section{Procedures for preparation 50 and 7.}
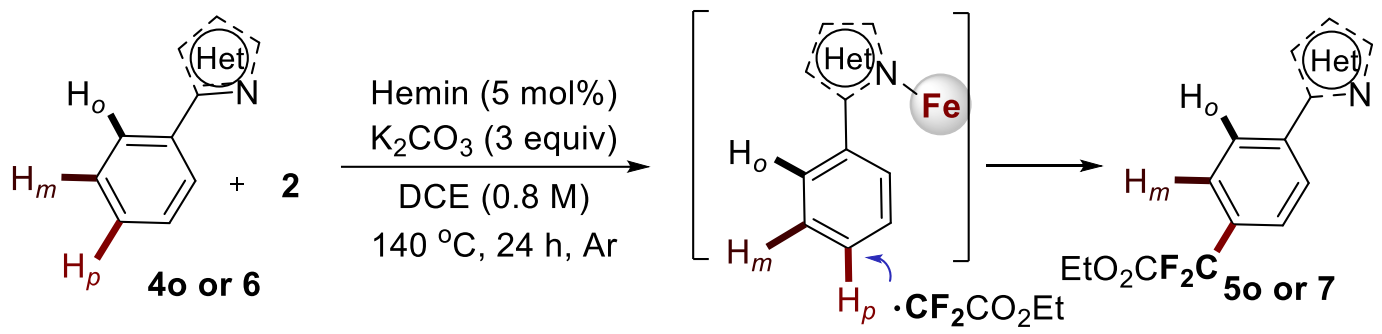

A mixture of 40 or 6 ( $0.2 \mathrm{mmol}, 1.0$ equiv), 2 ( $77 \mu \mathrm{L}, 121.2 \mathrm{mg}, 3$ equiv), Hemin (6.5 mg, $5 \mathrm{~mol} \%$ ), $\mathrm{K}_{2} \mathrm{CO}_{3}(81.6 \mathrm{mg}, 300 \mathrm{~mol} \%$ ), and DCE $(0.25 \mathrm{~mL})$ in a $15 \mathrm{~mL}$ glass vial sealed under argon atmosphere was heated at $140^{\circ} \mathrm{C}$ for 24 hours. The reaction mixture was cooled to room temperature and concentrated in vacuo. The resulting residue was purified by column chromatography (PE/EA $=20: 1)$ on silica gel to give the product 50 or 7 .<smiles>CCOC(=O)c1ccc(-c2ccccn2)cc1</smiles>

Yellow oil. Isolated yield: $19.4 \mathrm{mg}, 35 \%$.

${ }^{1} \mathrm{H}$ NMR (400 MHz, Chloroform- $d$ ) $\delta 8.72(\mathrm{~d}, J=4.0,1 \mathrm{H}), 8.08(\mathrm{~d}, J=8.0 \mathrm{~Hz}, 2 \mathrm{H}), 7.82-7.74(\mathrm{~m}, 2 \mathrm{H})$, $7.72(\mathrm{~d}, J=8.0 \mathrm{~Hz}, 2 \mathrm{H}), 7.28(\mathrm{ddd}, J=6.8,4.8,1.7 \mathrm{~Hz}, 1 \mathrm{H}), 4.31(\mathrm{q}, J=7.2 \mathrm{~Hz}, 2 \mathrm{H}), 1.59(\mathrm{~s}, 2 \mathrm{H}), 1.31$ $(\mathrm{t}, J=7.2 \mathrm{~Hz}, 3 \mathrm{H}) .{ }^{19} \mathrm{~F}$ NMR $\left(376 \mathrm{MHz}\right.$, Chloroform- $d$ ) $\delta-104.04 .{ }^{13} \mathrm{C}$ NMR $(100 \mathrm{MHz}$, Chloroform- $d$ ) $\delta 164.3\left(\mathrm{t}, J_{C-F}=35.4 \mathrm{~Hz}\right), 156.3,150.0,142.1,137.1,133.3\left(\mathrm{t}, J_{C-F}=25.8 \mathrm{~Hz}\right), 127.3,126.1\left(\mathrm{t}, J_{C-F}=\right.$ $6.1 \mathrm{~Hz}), 122.9,121.0,113.5\left(\mathrm{t}, J_{C-F}=253.5 \mathrm{~Hz}\right), 63.4,14.0$. HRMS Calcd for $\mathrm{C}_{15} \mathrm{H}_{13} \mathrm{~F}_{2} \mathrm{NO}_{2}\left[\mathrm{M}+\mathrm{H}^{+}\right]$: 278.0987, Found: 278.0983.<smiles>CCOC(=O)Cc1ccc(-c2ccc(C(F)(F)F)cc2)nc1</smiles>

Yellow oil. Isolated yield: $14.5 \mathrm{mg}, 18 \%$.

${ }^{1} \mathrm{H}$ NMR (400 MHz, Chloroform- $d$ ) $\delta 8.94(\mathrm{~s}, 1 \mathrm{H}), 8.12(\mathrm{~d}, J=8.0 \mathrm{~Hz}, 2 \mathrm{H}), 8.04-7.99(\mathrm{~m}, 1 \mathrm{H}), 7.85$ $(\mathrm{d}, J=8.0 \mathrm{~Hz}, 1 \mathrm{H}), 7.74(\mathrm{~d}, J=12.0 \mathrm{~Hz}, 2 \mathrm{H}), 4.41-4.21(\mathrm{~m}, 4 \mathrm{H}), 1.38-1.30(\mathrm{~m}, 6 \mathrm{H}) .{ }^{19} \mathrm{~F}$ NMR $(376$ 
MHz, Chloroform- $d$ ) $\delta$-104.14, -104.23 . HRMS Calcd for $\mathrm{C}_{19} \mathrm{H}_{17} \mathrm{~F}_{4} \mathrm{NO}_{4}\left[\mathrm{M}+\mathrm{H}^{+}\right]$: 400.1166, Found: 400.1169 .

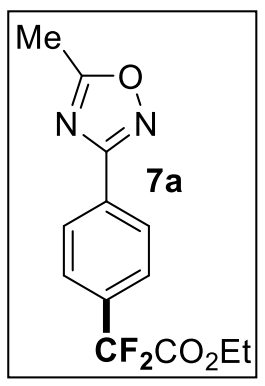

White solid. Isolated yield: $24.8 \mathrm{mg}, 44 \%$.

${ }^{1} \mathrm{H}$ NMR (400 MHz, Chloroform- $d$ ) $\delta 8.16(\mathrm{~d}, J=8.0 \mathrm{~Hz}, 2 \mathrm{H}), 7.73(\mathrm{~d}, J=8.0 \mathrm{~Hz}, 2 \mathrm{H}), 4.32(\mathrm{q}, J=7.2$ $\mathrm{Hz}, 2 \mathrm{H}), 2.67(\mathrm{~s}, 3 \mathrm{H}), 1.31(\mathrm{t}, J=7.2 \mathrm{~Hz}, 3 \mathrm{H}) .{ }^{19} \mathrm{~F}$ NMR $(376 \mathrm{MHz}$, Chloroform- $d) \delta-104.31 .{ }^{13} \mathrm{C}$ NMR $(100 \mathrm{MHz}$, Chloroform- $d) \delta 177.1,167.8,163.9\left(\mathrm{t}, J_{C-F}=34.9 \mathrm{~Hz}\right), 135.5\left(\mathrm{t}, J_{C-F}=25.6 \mathrm{~Hz}\right), 129.6(\mathrm{t}$, $\left.J_{C-F}=26.3 \mathrm{~Hz}\right), 127.8,126.3\left(\mathrm{t}, J_{C-F}=6.2 \mathrm{~Hz}\right), 113.2\left(\mathrm{t}, J_{C-F}=252.6 \mathrm{~Hz}\right), 63.5,14.0,12.5$. HRMS Calcd for $\mathrm{C}_{13} \mathrm{H}_{12} \mathrm{~F}_{2} \mathrm{~N}_{2} \mathrm{O}_{3}\left[\mathrm{M}+\mathrm{Na}^{+}\right]$: 305.0708, Found: 305.0716.<smiles>CCCc1nc(-c2ccc(C(=O)OCC)cc2)no1</smiles>

Yellow oil. Isolated yield: $24.8 \mathrm{mg}, 40 \%$.

${ }^{1} \mathrm{H}$ NMR (400 MHz, Chloroform- $d$ ) $\delta 8.17(\mathrm{~d}, J=8.0 \mathrm{~Hz}, 2 \mathrm{H}), 7.72(\mathrm{~d}, J=8.0 \mathrm{~Hz}, 2 \mathrm{H}), 4.31(\mathrm{q}, J=7.2$ $\mathrm{Hz}, 2 \mathrm{H}), 2.94(\mathrm{t}, J=4.0 \mathrm{~Hz}, 2 \mathrm{H}), 1.91(\mathrm{~h}, J=7.2 \mathrm{~Hz}, 2 \mathrm{H}), 1.30$ (t, $J=8.0 \mathrm{~Hz}, 3 \mathrm{H}), 1.06$ (t, $J=8.0 \mathrm{~Hz}$, $3 \mathrm{H}) .{ }^{19} \mathrm{~F}$ NMR (376 MHz, Chloroform- $d$ ) $\delta-104.36 .{ }^{13} \mathrm{C}$ NMR $(100 \mathrm{MHz}$, Chloroform- $d$ ) $\delta 180.5,167.6$, $164.0\left(\mathrm{t}, J_{C-F}=35.2 \mathrm{~Hz}\right), 135.4\left(\mathrm{t}, J_{C-F}=25.5 \mathrm{~Hz}\right), 129.8\left(\mathrm{t}, J_{C-F}=25.3 \mathrm{~Hz}\right), 127.8,126.2\left(\mathrm{t}, J_{C-F}=6.2\right.$ $\mathrm{Hz}), 113.2\left(\mathrm{t}, J_{C-F}=252.7 \mathrm{~Hz}\right), 63.5,28.6,20.3,14.0,13.7$. HRMS Calcd for $\mathrm{C}_{15} \mathrm{H}_{16} \mathrm{~F}_{2} \mathrm{~N}_{2} \mathrm{O}_{3}\left[\mathrm{M}+\mathrm{H}^{+}\right]$: 311.1202, Found: 311.1207.

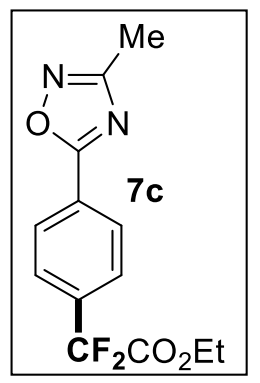

Yellow oil. Isolated yield: $21.4 \mathrm{mg}, 38 \%$.

${ }^{1} \mathrm{H}$ NMR (400 MHz, Chloroform- $d$ ) $\delta 8.20(\mathrm{~d}, J=12.0 \mathrm{~Hz}, 2 \mathrm{H}), 7.77(\mathrm{~d}, J=12.0 \mathrm{~Hz}, 2 \mathrm{H}), 4.32(\mathrm{q}, J=$ $7.2 \mathrm{~Hz}, 2 \mathrm{H}), 2.49$ (s, 3H), $1.31(\mathrm{t}, J=7.2 \mathrm{~Hz}, 3 \mathrm{H}) .{ }^{19} \mathrm{~F}$ NMR $(376 \mathrm{MHz}$, Chloroform- $d) \delta-104.56 .{ }^{13} \mathrm{C}$ NMR (100 MHz, Chloroform- $d$ ) $\delta 177.2,167.9,164.1\left(\mathrm{t}, J_{C-F}=34.9 \mathrm{~Hz}\right), 135.6\left(\mathrm{t}, J_{C-F}=25.6 \mathrm{~Hz}\right), 129.8$ 
$\left(\mathrm{t}, J_{C-F}=26.3 \mathrm{~Hz}\right), 127.9,126.4\left(\mathrm{t}, J_{C-F}=6.2 \mathrm{~Hz}\right), 113.3\left(\mathrm{t}, J_{C-F}=252.6 \mathrm{~Hz}\right), 63.6,14.1,12.6$. HRMS Calcd for $\mathrm{C}_{13} \mathrm{H}_{12} \mathrm{~F}_{2} \mathrm{~N}_{2} \mathrm{O}_{3}\left[\mathrm{M}+\mathrm{H}^{+}\right]$: 283.0889, Found: 283.0893.

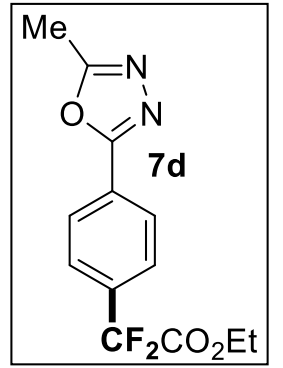

White solid. Isolated yield: $31.6 \mathrm{mg}, 56 \%$.

${ }^{1} \mathrm{H}$ NMR (400 MHz, Chloroform- $d$ ) $\delta 8.12(\mathrm{~d}, J=8.0 \mathrm{~Hz}, 2 \mathrm{H}), 7.75(\mathrm{~d}, J=8.0 \mathrm{~Hz}, 2 \mathrm{H}), 4.32(\mathrm{q}, J=7.2$ $\mathrm{Hz}, 2 \mathrm{H}), 2.64(\mathrm{~s}, 3 \mathrm{H}), 1.31(\mathrm{t}, J=7.2 \mathrm{~Hz}, 3 \mathrm{H}) .{ }^{19} \mathrm{~F}$ NMR $(376 \mathrm{MHz}$, Chloroform- $d) \delta-104.44 .{ }^{13} \mathrm{C}$ NMR $\left(100 \mathrm{MHz}\right.$, Chloroform- $d$ ) $\delta$ 164.3, 164.1, $163.8\left(\mathrm{t}, J_{C-F}=35.4 \mathrm{~Hz}\right), 135.9\left(\mathrm{t}, J_{C-F}=26.3 \mathrm{~Hz}\right), 127.1,126.6$ $\left(\mathrm{t}, J_{C-F}=2.0 \mathrm{~Hz}\right), 126.5\left(\mathrm{t}, J_{C-F}=6.2 \mathrm{~Hz}\right), 113.0\left(\mathrm{t}, J_{C-F}=253.0 \mathrm{~Hz}\right), 63.6,14.0,11.3$. HRMS Calcd for $\mathrm{C}_{13} \mathrm{H}_{12} \mathrm{~F}_{2} \mathrm{~N}_{2} \mathrm{O}_{3}\left[\mathrm{M}+\mathrm{H}^{+}\right]:$283.0889, Found: 283.0887.

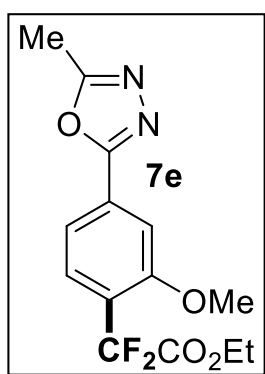

White solid. Isolated yield: $26.2 \mathrm{mg}, 42 \%$.

${ }^{1} \mathrm{H}$ NMR (400 MHz, Chloroform- $d$ ) $\delta 7.75(\mathrm{~d}, J=8.0 \mathrm{~Hz}, 1 \mathrm{H}), 7.65(\mathrm{dd}, J=8.0,1.2 \mathrm{~Hz}, 1 \mathrm{H}), 7.62(\mathrm{~s}$, $1 \mathrm{H}), 4.32$ (q, $J=7.2 \mathrm{~Hz}, 2 \mathrm{H}), 3.89$ (s, 3H), 2.62 (s, 3H), 1.28 (t, $J=7.2 \mathrm{~Hz}, 3 \mathrm{H}) .{ }^{19} \mathrm{~F}$ NMR (376 MHz, Chloroform- $d$ ) $\delta-103.40 .{ }^{13} \mathrm{C}$ NMR $\left(100 \mathrm{MHz}\right.$, Chloroform- $d$ ) $\delta 164.3,164.2,163.6\left(\mathrm{t}, J_{C-F}=33.5 \mathrm{~Hz}\right)$, $157.2\left(\mathrm{t}, J_{C-F}=4.7 \mathrm{~Hz}\right), 127.8,127.4\left(\mathrm{t}, J_{C-F}=7.6 \mathrm{~Hz}\right), 125.1\left(\mathrm{t}, J_{C-F}=24.3 \mathrm{~Hz}\right), 119.0,111.8\left(\mathrm{t}, J_{C-F}=\right.$ 249.3 Hz), 109.4, 63.0, 56.2, 14.0, 11.2. HRMS Calcd for $\mathrm{C}_{14} \mathrm{H}_{14} \mathrm{~F}_{2} \mathrm{~N}_{2} \mathrm{O}_{4}\left[\mathrm{M}+\mathrm{H}^{+}\right]$: 313.0994, Found: 313.0990 .<smiles>CCCc1nnc(-c2ccc(C(=O)OCC)cc2)o1</smiles>

Yellow oil. Isolated yield: $36.0 \mathrm{mg}, 58 \%$.

${ }^{1} \mathrm{H}$ NMR (400 MHz, Chloroform- $d$ ) $\delta 8.12(\mathrm{~d}, J=12.0 \mathrm{~Hz}, 2 \mathrm{H}), 7.74(\mathrm{~d}, J=8.0 \mathrm{~Hz}, 2 \mathrm{H}), 4.31(\mathrm{q}, J=7.2$ $\mathrm{Hz}, 2 \mathrm{H}), 2.92$ (t, $J=8.0 \mathrm{~Hz}, 2 \mathrm{H}), 1.89$ (h, $J=7.2 \mathrm{~Hz}, 2 \mathrm{H}), 1.39-1.26(\mathrm{~m}, 6 \mathrm{H}), 1.06$ (t, $J=7.2 \mathrm{~Hz}, 3 \mathrm{H})$. ${ }^{19}$ F NMR (376 MHz, Chloroform- $d$ ) $\delta-104.47 .{ }^{13} \mathrm{C}$ NMR (100 MHz, Chloroform- $d$ ) $\delta 167.5,163.9,163.8$ $\left(\mathrm{t}, J_{C-F}=34.9 \mathrm{~Hz}\right), 135.8\left(\mathrm{t}, J_{C-F}=25.8 \mathrm{~Hz}\right), 127.1,126.7\left(\mathrm{t}, J_{C-F}=2.5 \mathrm{~Hz}\right), 126.4\left(\mathrm{t}, J_{C-F}=6.1 \mathrm{~Hz}\right), 113.0$ $\left(\mathrm{t}, J_{C-F}=254.52 \mathrm{~Hz}\right), 63.6,27.5,20.2,14.0,13.8$. HRMS Calcd for $\mathrm{C}_{15} \mathrm{H}_{16} \mathrm{~F}_{2} \mathrm{~N}_{2} \mathrm{O}_{3}\left[\mathrm{M}+\mathrm{H}^{+}\right]: 311.1202$, Found: 311.1207. 


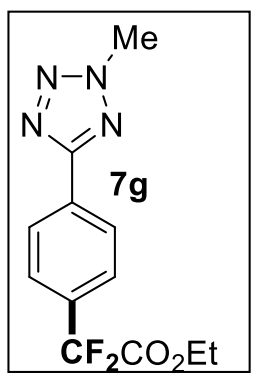

White solid. Isolated yield: $36.1 \mathrm{mg}, 64 \%$.

${ }^{1} \mathrm{H}$ NMR (400 MHz, Chloroform- $d$ ) $\delta 8.23(\mathrm{~d}, J=12.0 \mathrm{~Hz}, 2 \mathrm{H}), 7.73(\mathrm{~d}, J=8.0 \mathrm{~Hz}, 2 \mathrm{H}), 4.41(\mathrm{~s}, 3 \mathrm{H})$, $4.31(\mathrm{q}, J=7.2 \mathrm{~Hz}, 2 \mathrm{H}), 1.31(\mathrm{t}, J=7.2 \mathrm{~Hz}, 3 \mathrm{H}) .{ }^{19} \mathrm{~F}$ NMR $(376 \mathrm{MHz}$, Chloroform- $d) \delta-104.19 .{ }^{13} \mathrm{C}$ $\operatorname{NMR}(100 \mathrm{MHz}$, Chloroform- $d) \delta 164.5,164.0\left(\mathrm{t}, J_{C-F}=35.0 \mathrm{~Hz}\right), 134.7\left(\mathrm{t}, J_{C-F}=25.8 \mathrm{~Hz}\right), 130.1,127.2$, $126.3\left(\mathrm{t}, J_{C-F}=6.2 \mathrm{~Hz}\right), 113.3\left(\mathrm{t}, J_{C-F}=252.5 \mathrm{~Hz}\right), 63.4,39.7,14.0$. HRMS Calcd for $\mathrm{C}_{12} \mathrm{H}_{12} \mathrm{~F}_{2} \mathrm{~N}_{4} \mathrm{O}_{2}$ $\left[\mathrm{M}+\mathrm{H}^{+}\right]: 283.1001$, Found: 283.1006.

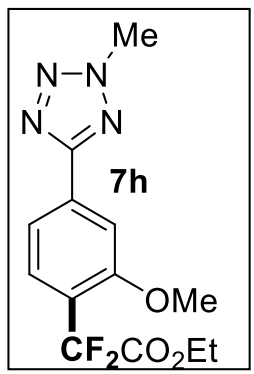

White solid. Isolated yield: $35.6 \mathrm{mg}, 57 \%$.

${ }^{1} \mathrm{H}$ NMR (400 MHz, Chloroform- $d$ ) $\delta 7.82(\mathrm{~d}, J=8.0 \mathrm{~Hz}, 1 \mathrm{H}), 7.75(\mathrm{~d}, J=8.0 \mathrm{~Hz}, 1 \mathrm{H}), 7.72(\mathrm{~s}, 1 \mathrm{H})$, $4.40(\mathrm{~s}, 3 \mathrm{H}), 4.33(\mathrm{q}, J=7.1 \mathrm{~Hz}, 2 \mathrm{H}), 3.91(\mathrm{~s}, 3 \mathrm{H}), 1.29(\mathrm{t}, J=7.1 \mathrm{~Hz}, 3 \mathrm{H}) .{ }^{19} \mathrm{~F}$ NMR $(376 \mathrm{MHz}$, Chloroform- $d$ ) $\delta$-103.04. ${ }^{13} \mathrm{C}$ NMR (100 MHz, Chloroform- $d$ ) $\delta 164.5,163.9\left(\mathrm{t}, J_{C-F}=33.8 \mathrm{~Hz}\right), 157.3$ $\left(\mathrm{t}, J_{C-F}=4.8 \mathrm{~Hz}\right), 131.4,127.3\left(\mathrm{t}, J_{C-F}=7.5 \mathrm{~Hz}\right), 123.9\left(\mathrm{t}, J_{C-F}=24.2 \mathrm{~Hz}\right), 119.2,112.1\left(\mathrm{t}, J_{C-F}=249.5\right.$ $\mathrm{Hz}), 109.7,62.9,56.1,39.7,14.0$. HRMS Calcd for $\mathrm{C}_{13} \mathrm{H}_{14} \mathrm{~F}_{2} \mathrm{~N}_{4} \mathrm{O}_{4}\left[\mathrm{M}+\mathrm{H}^{+}\right]$: 313.1107, Found: 313.1103.

\section{Procedures for preparation 9.}<smiles>[2H]c1ccc2c(c1)CCN2C</smiles><smiles>CCOC(=O)C(Br)(Br)Br</smiles>

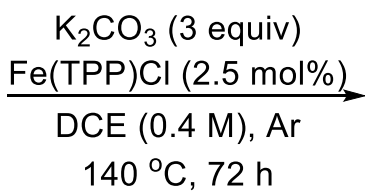

$140{ }^{\circ} \mathrm{C}, 72 \mathrm{~h}$

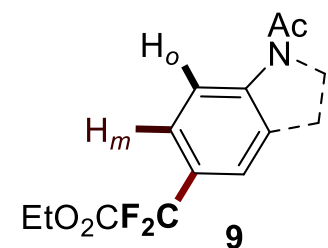

A mixture of 8 (0.2 mmol, 1.0 equiv), 2 (154 $\mu \mathrm{L}, 242.4 \mathrm{mg}, 6$ equiv), $\mathrm{Fe}(\mathrm{Tpp}) \mathrm{Cl}(3.5 \mathrm{mg}, 2.5 \mathrm{~mol} \%$ ), $\mathrm{K}_{2} \mathrm{CO}_{3}(81.6 \mathrm{mg}, 300 \mathrm{~mol} \%)$, and DCE $(0.5 \mathrm{~mL})$ in a $15 \mathrm{~mL}$ glass vial sealed under argon atmosphere was heated at $140{ }^{\circ} \mathrm{C}$ for 72 hours. The reaction mixture was cooled to room temperature and concentrated in vacuo. The resulting residue was purified by column chromatography (PE/EA $=4: 1)$ on silica gel to give the product 9 . 


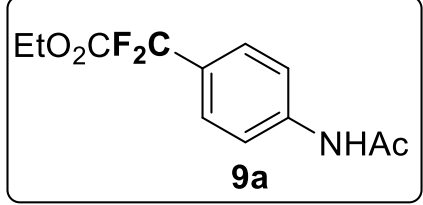

yellow oil. Isolated yield: $39.2 \mathrm{mg}, 76 \%$.

${ }^{1} \mathrm{H}$ NMR (400 MHz, CDCl $) \delta 7.60-7.58(\mathrm{~m}, 2 \mathrm{H}), 7.54(\mathrm{~d}, J=8.0 \mathrm{~Hz}, 2 \mathrm{H}), 7.51(\mathrm{~s}, 1 \mathrm{H}), 4.29$ (q, $J$ $=7.2 \mathrm{~Hz}, 2 \mathrm{H}), 2.19(\mathrm{~s}, 3 \mathrm{H}), 1.30(\mathrm{t}, J=7.2 \mathrm{~Hz}, 3 \mathrm{H}) .{ }^{19} \mathrm{~F}$ NMR $(377 \mathrm{MHz}, \mathrm{CDCl} 3) \delta-103.29 .{ }^{13} \mathrm{C}$ $\operatorname{NMR}\left(101 \mathrm{MHz}, \mathrm{CDCl}_{3}\right) \delta 168.7,164.4\left(\mathrm{t}, J_{C-F}=35.7 \mathrm{~Hz}\right), 140.5,128.4\left(\mathrm{t}, J_{C-F}=26.7 \mathrm{~Hz}\right), 126.6$ $\left(\mathrm{d}, J_{C-F}=6.2 \mathrm{~Hz}\right), 119.6,113.4\left(\mathrm{t}, J_{C-F}=251.9 \mathrm{~Hz}\right), 63.3,24.8,14.0$. HRMS Calcd for $\mathrm{C}_{12} \mathrm{H}_{13} \mathrm{~F}_{2} \mathrm{NO}_{3}$ $\left[\mathrm{M}+\mathrm{H}^{+}\right]$: 258.0936, Found: 258.0939.<smiles>CCOC(F)(F)C(F)(F)F</smiles>

yellow solid. Isolated yield: $53.6 \mathrm{mg}, 80 \%$.

1H NMR (400 MHz, CDCl3) $\delta 7.93$ (s, 1H), 7.63 (d, $J=8.0 \mathrm{~Hz}, 1 \mathrm{H}), 7.57$ (s, 1H), 7.49 (d, $J=8.0 \mathrm{~Hz}$, 1H), 4.35 (q, $J=7.2 \mathrm{~Hz}, 2 \mathrm{H}), 2.19$ (s, 3H), 1.32 (t, $J=7.2 \mathrm{~Hz}, 3 \mathrm{H}) .{ }^{19} \mathrm{~F}$ NMR $(377 \mathrm{MHz}, \mathrm{CDCl} 3) \delta-$ 101.01. 13C NMR (101 MHz, CDCl3) $\delta 168.8,163.4\left(\mathrm{t}, J_{C-F}=34.2 \mathrm{~Hz}\right), 141.2,128.3\left(\mathrm{t}, J_{C-F}=8.9 \mathrm{~Hz}\right)$, 128.2, 124.5, $120.9\left(\mathrm{t}, J_{C-F}=4.2 \mathrm{~Hz}\right), 117.9,112.9\left(\mathrm{t}, J_{C-F}=240 \mathrm{~Hz}\right), 63.61,24.75,13.94$. HRMS Calcd for $\mathrm{C}_{12} \mathrm{H}_{12} \mathrm{BrF}_{2} \mathrm{NO}_{3}\left[\mathrm{M}+\mathrm{H}^{+}\right]$: 336.0041, Found: 336.0046 .<smiles>CCOC(F)(F)C(F)(F)F</smiles>

yellow oil. Isolated yield: $31.1 \mathrm{mg}, 55 \%$.

${ }^{1} \mathrm{H}$ NMR $\left(400 \mathrm{MHz}, \mathrm{CDCl}_{3}\right) \delta 8.06(\mathrm{~s}, 1 \mathrm{H}), 7.85(\mathrm{~d}, J=8.0 \mathrm{~Hz}, 1 \mathrm{H}), 7.72(\mathrm{~s}, 1 \mathrm{H}), 7.70-7.67(\mathrm{~m}, 1 \mathrm{H})$, $4.36(\mathrm{q}, J=7.2 \mathrm{~Hz}, 2 \mathrm{H}), 2.23$ (s, 3H), $1.34(\mathrm{t}, J=7.2 \mathrm{~Hz}, 3 \mathrm{H}) .{ }^{19} \mathrm{~F}$ NMR $\left(377 \mathrm{MHz}, \mathrm{CDCl}_{3}\right) \delta-100.95$. ${ }^{13} \mathrm{C}$ NMR $\left(101 \mathrm{MHz}, \mathrm{CDCl}_{3}\right) \delta 169.0,162.9\left(\mathrm{t}, J_{C-F}=34.4 \mathrm{~Hz}\right), 141.0,130.4,128.2\left(\mathrm{t}, J_{C-F}=7.5 \mathrm{~Hz}\right)$, 124.9, 123.0, 116.0, $112.3\left(\mathrm{t}, J_{C-F}=253 \mathrm{~Hz}\right), 111.3\left(\mathrm{t}, J_{C-F}=3.7 \mathrm{~Hz}\right), 64.1,24.7,14.0$. HRMS Calcd for $\mathrm{C}_{13} \mathrm{H}_{12} \mathrm{~F}_{2} \mathrm{~N}_{2} \mathrm{O}_{3}\left[\mathrm{M}+\mathrm{H}^{+}\right]: 283.0889$, Found: 283.0881 .

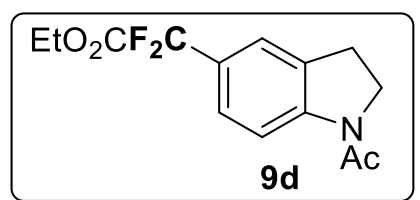

yellow oil. Isolated yield: $48.3 \mathrm{mg}, 85 \%$

${ }^{1} \mathrm{H} \mathrm{NMR}\left(400 \mathrm{MHz}, \mathrm{CDCl}_{3}\right) \delta 8.25(\mathrm{~d}, J=8.3 \mathrm{~Hz}, 1 \mathrm{H}), 7.43-7.40(\mathrm{~m}, 2 \mathrm{H}), 4.28(\mathrm{q}, J=7.2 \mathrm{~Hz}, 2 \mathrm{H}), 4.09$ (t, $J=8.0 \mathrm{~Hz}, 2 \mathrm{H}), 3.22$ (t, $J=8.0 \mathrm{~Hz}, 2 \mathrm{H}), 2.23$ (s, 3H), 1.29 (t, $J=8.0 \mathrm{~Hz}, 3 \mathrm{H}) .{ }^{19} \mathrm{~F}$ NMR $(377 \mathrm{MHz}$, $\left.\mathrm{CDCl}_{3}\right) \delta-102.75 .{ }^{13} \mathrm{C} \mathrm{NMR}\left(101 \mathrm{MHz}, \mathrm{CDCl}_{3}\right) \delta 169.2,164.5\left(\mathrm{t}, J_{C-F}=35.6 \mathrm{~Hz}\right), 145.34,131.84,127.9$ $\left(\mathrm{d}, J_{C-F}=51.7 \mathrm{~Hz}\right), 125.6\left(\mathrm{t}, J_{C-F}=6.1 \mathrm{~Hz}\right), 121.9\left(\mathrm{t}, J_{C-F}=6.0 \mathrm{~Hz}\right), 116.8,113.6\left(\mathrm{t}, J_{C-F}=251.7 \mathrm{~Hz}\right)$, 63.2, 49.1, 27.9, 24.3, 14.0. HRMS Calcd for $\mathrm{C}_{14} \mathrm{H}_{15} \mathrm{~F}_{2} \mathrm{NO}_{3}\left[\mathrm{M}+\mathrm{H}^{+}\right]$: 284.1093, Found: 284.1097 . 


\section{Procedures for preparation 5q.}

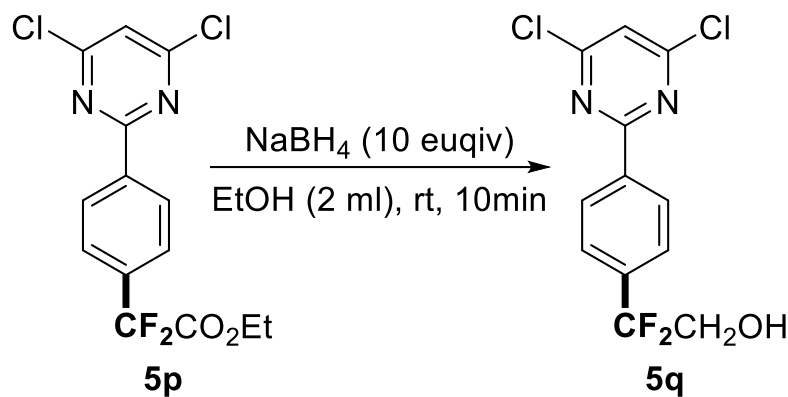

In a $15 \mathrm{~mL}$ glass vial, a mixture of $\mathbf{5 p}\left(0.2 \mathrm{mmol}, 1\right.$ equiv), $\mathrm{NaBH}_{4}$ (10 equiv), EtOH ( $\left.2 \mathrm{~mL}\right)$ and stirred for $10 \mathrm{~min}$ at room temperature. The reaction was then poured into $1 \mathrm{M} \mathrm{HCl}$ aq. to acidify to $\mathrm{pH} 1$, and the aqueous phase was extracted with EtOAc $(100 \mathrm{~mL})$, washed with water $(100 \mathrm{~mL})$, dried over $\mathrm{Na}_{2} \mathrm{SO}_{4}$ and concentrated in vacuo. Product $5 \mathbf{q}$ was purified by flash chromatography on silica gel with $\mathrm{PE} / \mathrm{EA}=5 / 1$ as the eluent.<smiles>OCc1ccc(-c2nc(Cl)cc(Cl)n2)cc1</smiles>

White solid. Isolated yield: $49.9 \mathrm{mg}, 82 \%$.

${ }^{1} \mathrm{H}$ NMR (400 MHz, Chloroform- $d$ ) $\delta 8.47(\mathrm{~d}, J=8.0 \mathrm{~Hz}, 2 \mathrm{H}), 7.62(\mathrm{~d}, J=8.0 \mathrm{~Hz}, 2 \mathrm{H}), 7.30(\mathrm{~s}, 1 \mathrm{H})$, $3.99(\mathrm{t}, J=12.0 \mathrm{~Hz}, 2 \mathrm{H}), 2.43(\mathrm{~s}, 1 \mathrm{H}) \cdot{ }^{19} \mathrm{~F}$ NMR $\left(376 \mathrm{MHz}\right.$, Chloroform- $d$ ) $\delta-107.46 \cdot{ }^{13} \mathrm{C}$ NMR $(100$ MHz, Chloroform- $d$ ) $\delta 164.8,162.3,138.1\left(\mathrm{t}, J_{C-F}=25.8 \mathrm{~Hz}\right), 136.8,129.1,126.1\left(\mathrm{t}, J_{C-F}=6.2 \mathrm{~Hz}\right)$, $120.5\left(\mathrm{t}, J_{C-F}=244.3 \mathrm{~Hz}\right), 119.5,65.9\left(\mathrm{t}, J_{C-F}=32.4 \mathrm{~Hz}\right)$. HRMS Calcd for $\mathrm{C}_{12} \mathrm{H}_{8} \mathrm{C}_{12} \mathrm{~F}_{2} \mathrm{~N}_{2} \mathrm{O}\left[\mathrm{M}+\mathrm{H}^{+}\right]$: 305.0055, Found: 305.0061 .

\section{Scale up synthesis of $5 i$ and procedures for preparation $5 r$.}

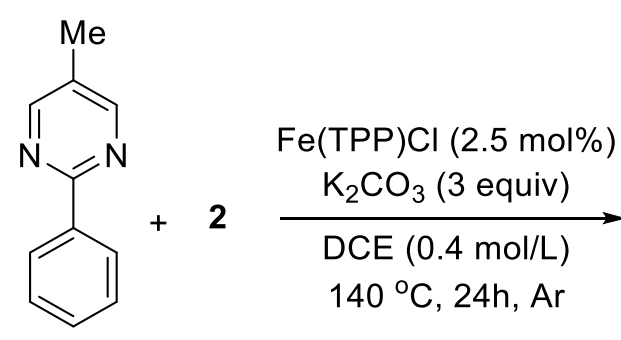

$4 \mathbf{i}$<smiles>CCOC(=O)C(F)(F)c1ccc(-c2ncc(C)cn2)cc1</smiles>

5i, $62 \%, \mathrm{p} / \mathrm{m} \mathrm{10:1}$

A mixture of $4 \mathbf{i}(1 \mathrm{mmol}, 1.0$ equiv), 2 (770 $\mu \mathrm{L}, 1218 \mathrm{mg}, 6$ equiv), $\mathrm{Fe}(\mathrm{Tpp}) \mathrm{Cl}(17.5 \mathrm{mg}, 2.5 \mathrm{~mol} \%)$, 
$\mathrm{K}_{2} \mathrm{CO}_{3}(415 \mathrm{mg}, 300 \mathrm{~mol} \%$ ), and DCE $(2.5 \mathrm{~mL})$ in a $25 \mathrm{~mL}$ glass vial sealed under argon atmosphere was heated at $140{ }^{\circ} \mathrm{C}$ for 24 hours. The reaction mixture was cooled to room temperature and concentrated in vacuo. The resulting residue was purified by column chromatography (PE/EA $=10: 1)$ on silica gel to give the product $\mathbf{5 i}$ as white solids in $62 \%($ Para/ Meta $=10: 1)$ yield $(181$ $\mathrm{mg})$.<smiles>Cc1ccc(-c2ncc(C)cn2)nc1</smiles>

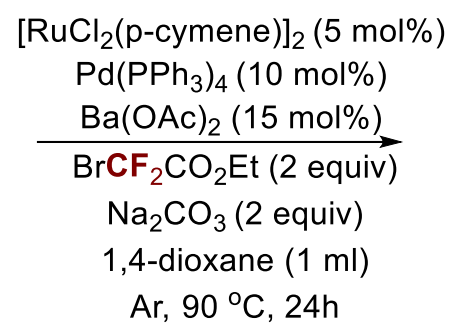<smiles>CCOC(=O)c1cccc(-c2ncc(C)cn2)c1</smiles>

5r, 70\%, p/m 1:15

To a $15 \mathrm{~mL}$ of Schlenck flask were added $4 \mathbf{i}\left(0.2 \mathrm{mmol}, 1.0 \text { equiv), [ } \mathrm{RuCl}_{2}(\mathrm{p} \text {-cymene })\right]_{2}(0.01 \mathrm{mmol}$, $5 \mathrm{~mol} \%), \mathrm{Pd}\left(\mathrm{PPh}_{3}\right)_{4}(0.02 \mathrm{mmol}, 10 \mathrm{~mol} \%)$ under air, followed by $\mathrm{Na}_{2} \mathrm{CO}_{3}(0.4 \mathrm{mmol}, 2.0$ equiv $)$ and $\mathrm{Ba}(\mathrm{OAc})_{2}$ ( 0.03 mmol, 0.15 equiv). The mixture was then evacuated and backfilled with nitrogen for three times. After that, $\mathrm{BrCF}_{2} \mathrm{CO}_{2} \mathrm{Et}(0.4 \mathrm{mmol}, 2.0$ equiv) and 1,4-dioxane $(1 \mathrm{~mL})$ were added subsequently. After stirring at $90{ }^{\circ} \mathrm{C}$ for $24 \mathrm{~h}$, the reaction mixture was cooled to room temperature. The solvent was removed under reduced pressure, and the residue was purified by flash chromatography on silica gel with petroleum ether/ethyl acetate as the eluent to give the corresponding product $5 \mathbf{r}$ as yellow solids in 70\% (Meta/Para $=15: 1)$ yield $(41 \mathrm{mg})$.

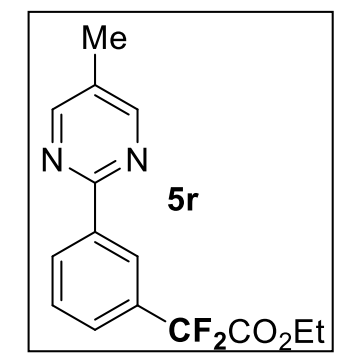

${ }^{1} \mathrm{H}$ NMR (400 MHz, Chloroform- $d$ ) $\delta 8.69(\mathrm{~s}, 1 \mathrm{H}), 8.62(\mathrm{~s}, 2 \mathrm{H}), 8.53(\mathrm{~d}, J=8.0 \mathrm{~Hz}, 1 \mathrm{H}), 7.69(\mathrm{~d}, J=8.0$ $\mathrm{Hz}, 1 \mathrm{H}), 7.55(\mathrm{t}, J=8.0 \mathrm{~Hz}, 1 \mathrm{H}), 4.30(\mathrm{q}, J=7.2 \mathrm{~Hz}, 2 \mathrm{H}), 2.33(\mathrm{~s}, 3 \mathrm{H}), 1.29(\mathrm{t}, J=7.2 \mathrm{~Hz}, 3 \mathrm{H}) .{ }^{19} \mathrm{~F}$ NMR $\left(376 \mathrm{MHz}\right.$, Chloroform- $d$ ) $\delta-103.51 .{ }^{13} \mathrm{C}$ NMR $\left(100 \mathrm{MHz}\right.$, Chloroform- $d$ ) $\delta 164.3\left(\mathrm{t}, J_{C-F}=35.2\right.$ $\mathrm{Hz}), 161.4,157.6,138.4,133.4\left(\mathrm{t}, J_{C-F}=25.6 \mathrm{~Hz}\right), 130.4,129.1,129.0,127.3\left(\mathrm{t}, J_{C-F}=6.0 \mathrm{~Hz}\right), 125.1(\mathrm{t}$, $\left.J_{C-F}=6.3 \mathrm{~Hz}\right), 113.5\left(\mathrm{t}, J_{C-F}=252.2 \mathrm{~Hz}\right), 63.3,15.6,14.0$. 


\section{General Procedures for TEMPO Inhibition Experiments.}

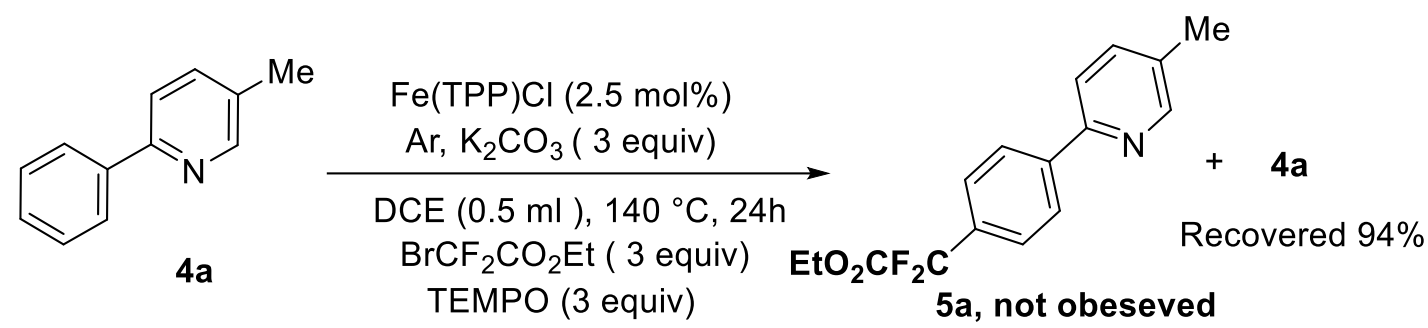

A mixture of $4 \mathbf{a}$ ( $0.2 \mathrm{mmol}, 1.0$ equiv), $\mathrm{BrCF}_{2} \mathrm{CO}_{2} \mathrm{Et}$ ( $77 \mu \mathrm{L}, 121.2 \mathrm{mg}, 3$ equiv), $\mathrm{Fe}(\mathrm{TPP}) \mathrm{Cl}$ (3.5 $\mathrm{mg}, 2.5 \mathrm{~mol} \%), \mathrm{K}_{2} \mathrm{CO}_{3}(81.6 \mathrm{mg}, 300 \mathrm{~mol} \%$ ), TEMPO (93.7 mg, 3 equiv) and DCE (0.5 mL) in a $15 \mathrm{~mL}$ glass vial sealed under argon atmosphere was heated at $140{ }^{\circ} \mathrm{C}$ for 24 hours. Totally no reaction.

\section{Radical trapping experiments}

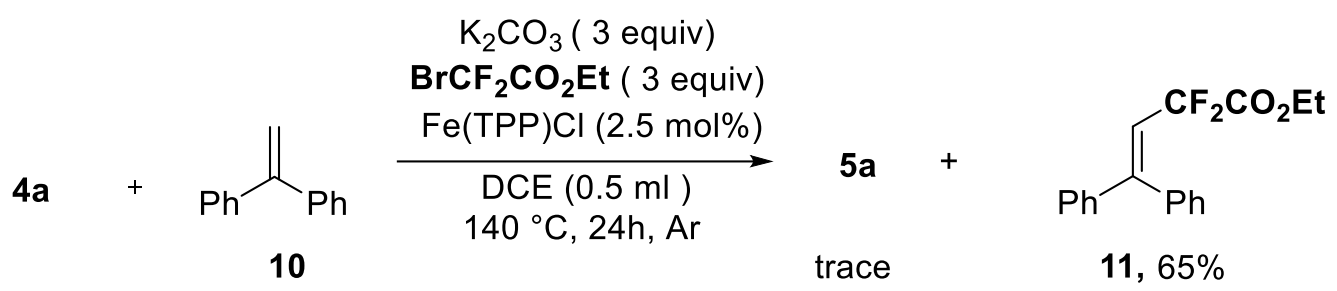

A mixture of $4 \mathbf{a}\left(0.2 \mathrm{mmol}, 1.0\right.$ equiv), 8 ( $0.2 \mathrm{mmol}, 1.0$ equiv), $\mathrm{BrCF}_{2} \mathrm{CO}_{2} \mathrm{Et}(77 \mu \mathrm{L}, 121.2 \mathrm{mg}, 3$ equiv), $\mathrm{Fe}(\mathrm{TPP}) \mathrm{Cl}(3.5 \mathrm{mg}, 2.5 \mathrm{~mol} \%), \mathrm{K}_{2} \mathrm{CO}_{3}(81.6 \mathrm{mg}, 300 \mathrm{~mol} \%$ ), and DCE $(0.5 \mathrm{~mL})$ in a 15 $\mathrm{mL}$ glass vial sealed under argon atmosphere was heated at $140{ }^{\circ} \mathrm{C}$ for 24 hours. The reaction mixture was cooled to room temperature and concentrated in vacuo. The resulting residue was purified by column chromatography $(\mathrm{PE} / \mathrm{EA}=10: 1)$ on silica gel to give the product $\mathbf{1 1}$.

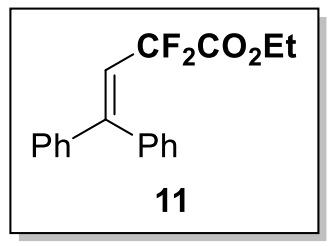

Yellow oil. Isolated yield: $39.3 \mathrm{mg}, 65 \%$.

${ }^{1} \mathrm{H}$ NMR (400 MHz, Chloroform- $d$ ) $\delta 7.41-7.27$ (m, 6H), $7.30-7.22$ (m, 2H), 7.20 (dt, $J=4.9,3.5$ $\mathrm{Hz}, 2 \mathrm{H}), 6.27(\mathrm{t}, J=12.0 \mathrm{~Hz}, 1 \mathrm{H}), 3.91(\mathrm{q}, J=7.2 \mathrm{~Hz}, 2 \mathrm{H}), 1.17(\mathrm{t}, J=7.2 \mathrm{~Hz}, 3 \mathrm{H})$. 


\section{KIE by two parallel reactions}

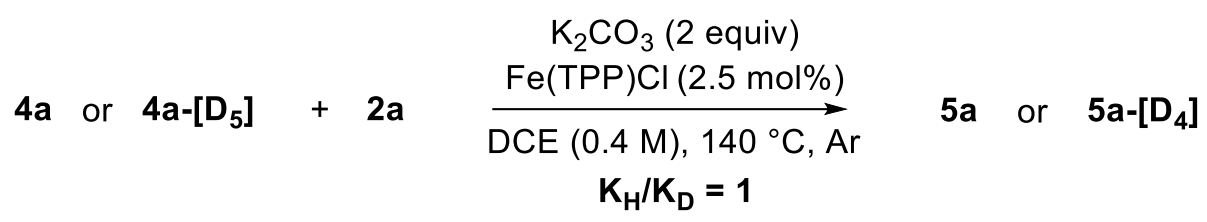

KIE by two parallel reactions: A mixture of $\mathbf{4 a}$ or $\mathbf{4 a}-\left[\mathrm{D}_{5}\right]$ ( $0.2 \mathrm{mmol}, 1.0$ equiv), $\mathrm{BrCF}_{2} \mathrm{CO}_{2} \mathrm{Et}$ (160 $\mu \mathrm{L}, 242.4 \mathrm{mg}, 6$ equiv), $\mathrm{Fe}(\mathrm{Tpp}) \mathrm{Cl}$ (3.5 mg, $2.5 \mathrm{~mol} \%), \mathrm{K}_{2} \mathrm{CO}_{3}(55.2 \mathrm{mg}, 200 \mathrm{~mol} \%$ ), and DCE $(0.5 \mathrm{~mL})$ in a $15 \mathrm{~mL}$ glass vial sealed under argon atmosphere was heated at $140{ }^{\circ} \mathrm{C}$. $\mathrm{GC}$ analysis using $\mathrm{n}$-Tridecane as an internal standard to provide the following conversions.

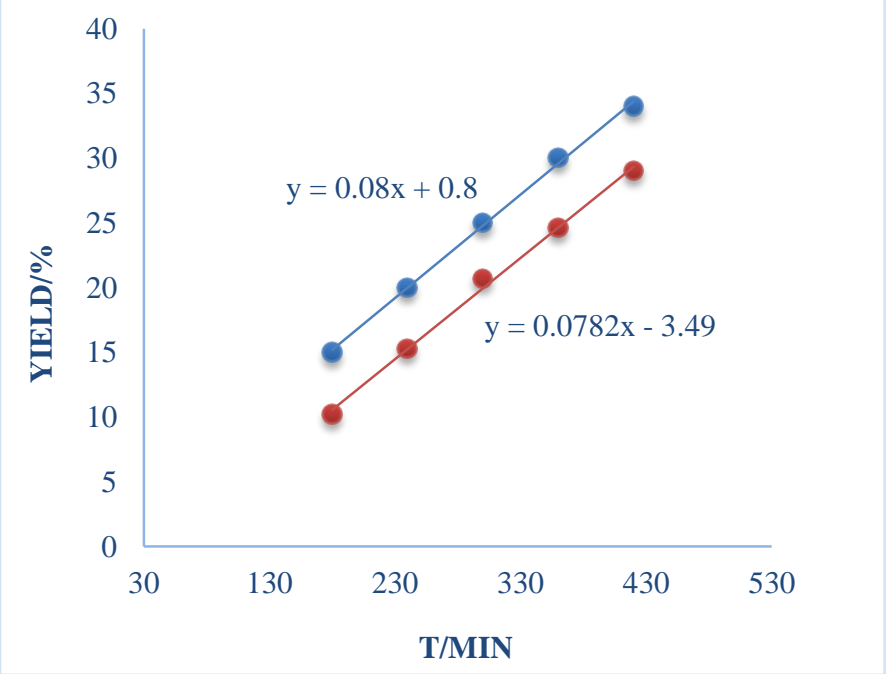

\section{KIE by intermolecular competion}

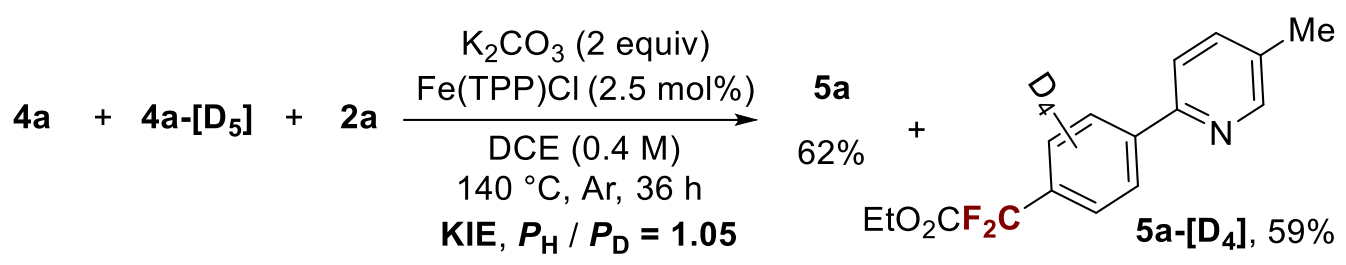

KIE by intermolecular competion: A mixture of $\mathbf{4 a}(0.1 \mathrm{mmol})$ and $\mathbf{4 a}-\left[\mathrm{D}_{5}\right](0.1 \mathrm{mmol})$, $\mathrm{BrCF}_{2} \mathrm{CO}_{2} \mathrm{Et}(160 \mu \mathrm{L}, 242.4 \mathrm{mg}), \mathrm{Fe}(\mathrm{Tpp}) \mathrm{Cl}(0.005 \mathrm{mmol}, 3.5 \mathrm{mg}), \mathrm{K}_{2} \mathrm{CO}_{3}(0.4 \mathrm{mmol}, 55 \mathrm{mg})$, and DCE $(0.5 \mathrm{~mL})$ in a $15 \mathrm{~mL}$ glass vial sealed under argon atmosphere was heated at $140{ }^{\circ} \mathrm{C}$ for $36 \mathrm{~h}$. The resulting residue was purified by column chromatography $(\mathrm{PE} / \mathrm{EA}=20: 1)$ on silica gel to give the products. The results determined by ${ }^{1} \mathrm{H}$ NMR spectroscopy. 


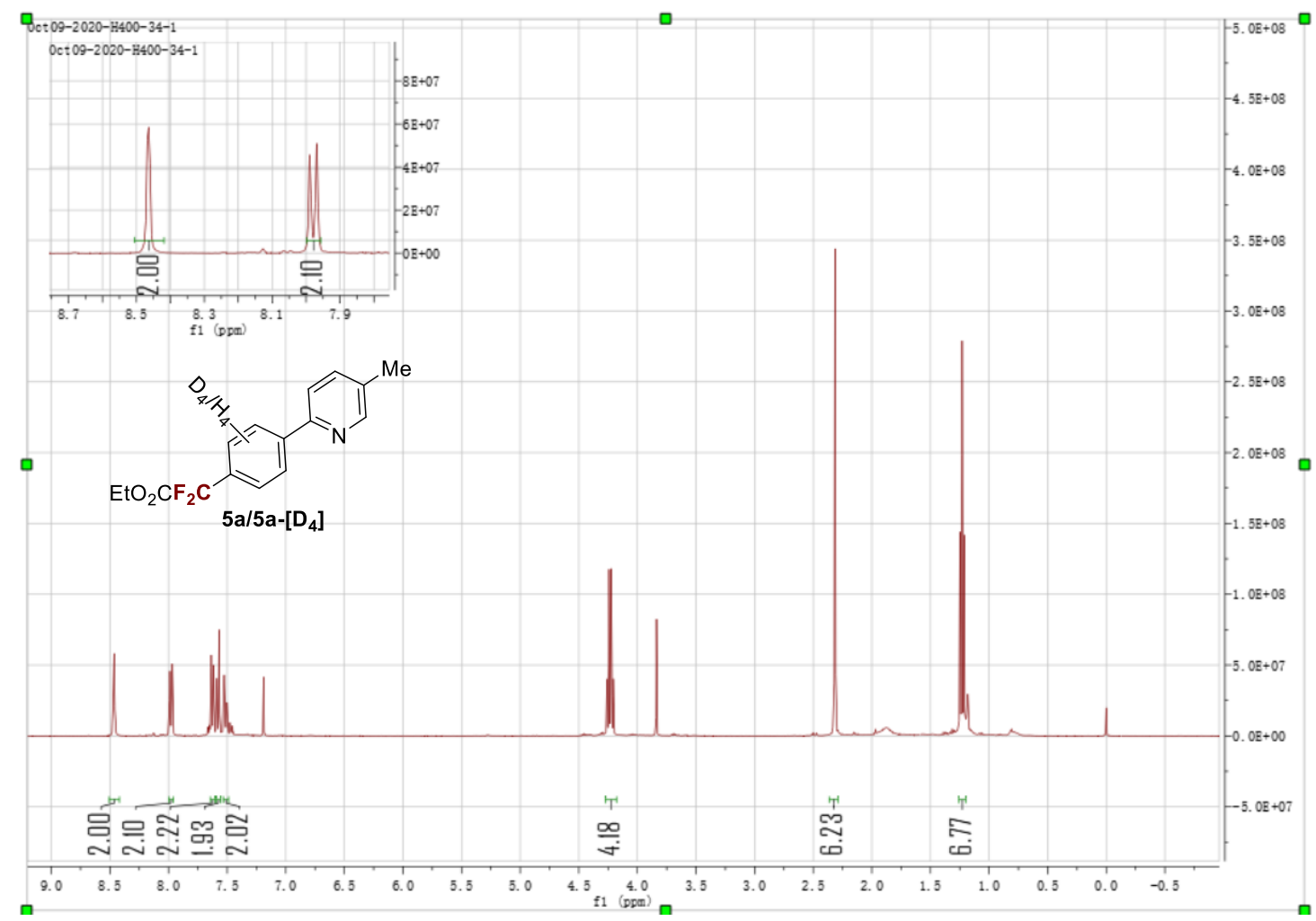

\section{The characterization of $\mathrm{Fe}^{2+}$ by XPS}

The substrate $4 \mathbf{a}$ was treated with $\mathbf{2}$ in presence of $\mathrm{Fe}(\mathrm{TPP}) \mathrm{Cl}$ for $3 \mathrm{hrs}$ under argon in DCE. The reaction mixture was directed dried by oil pump, and then transformed into glove box. The reaction mixture was direct by X-ray photoelectron spectroscopy. The result listed below.

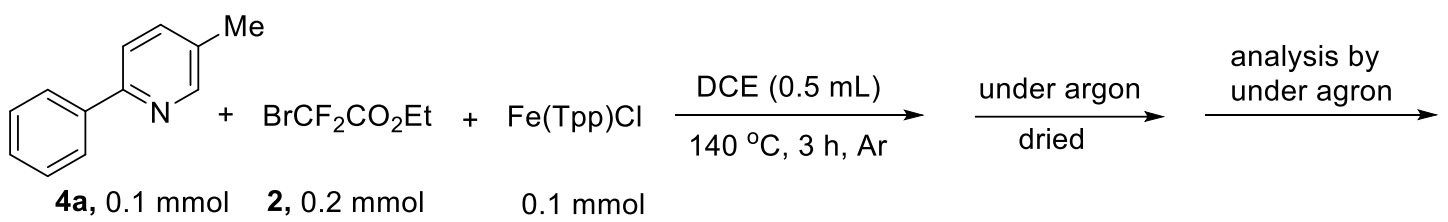

In order to explain the mechanism more favorably, the XPS spectrum was used to further analyze the catalyst residue after the reaction, and the photoelectron peaks at 711.4 and $724.9 \mathrm{eV}$ correspond to $\mathrm{Fe} 2 \mathrm{p}_{3 / 2}$ and $\mathrm{Fe} 2 \mathrm{p}_{1 / 2}$, respectively. It shows that in addition to $\mathrm{Fe}^{3+}, \mathrm{Fe}^{2+}$ also exists in the catalytic system $^{6}$. And then the two peaks at 711.4 and $724.9 \mathrm{eV}$ split into four peaks, two of them at 711.3 and $724.8 \mathrm{eV}$ ascribing to the $\mathrm{Fe} 2 \mathrm{p}_{3 / 2}$ and $\mathrm{Fe} 2 \mathrm{p}_{1 / 2}$ orbits of $\mathrm{Fe}^{2+}$, and the other two peaks at 713.6 and $727.8 \mathrm{eV}$ corresponding to the $\mathrm{Fe} 2 \mathrm{p}_{3 / 2}$ and $\mathrm{Fe} 2 \mathrm{p}_{1 / 2}$ orbits of $\mathrm{Fe}^{3+}$. 


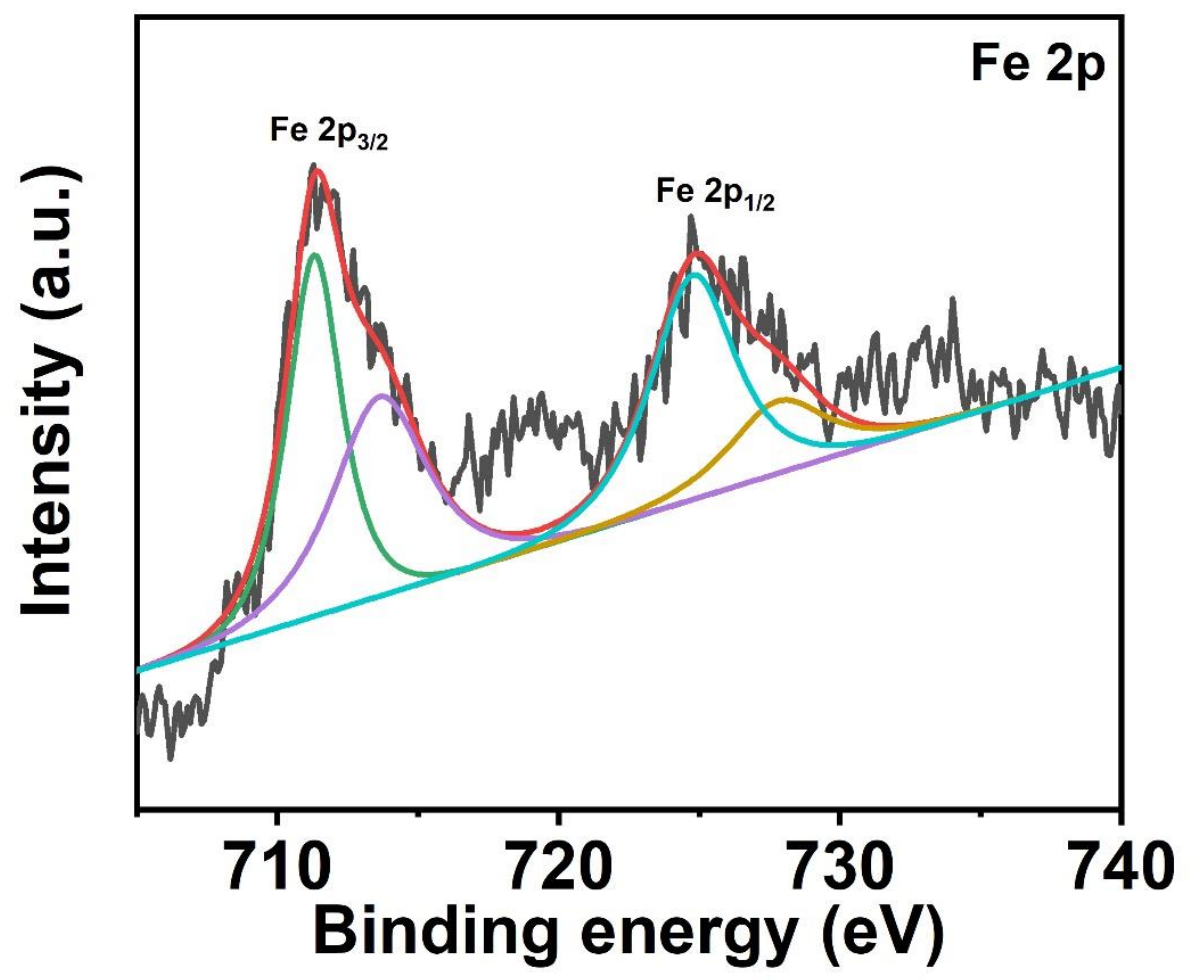

\section{Cyclic voltammetry}

Experimental Section:

Cyclic voltammetry measurements were performed on CHI660D Electrochemical Workstation at $50 \mathrm{mVs}^{-1}$, with $\mathrm{Ag} / \mathrm{AgCl}$ (saturated $\mathrm{KCl}$ ) as the reference electrode, glassy carbon disk ( $\varnothing=3 \mathrm{~mm}$ ) as working electrode, and platinum wire as the counter electrode. The solution containing all the regents and catalyst $(\mathbf{4 a}(0.2 \mathrm{mmol}), 2(1.2 \mathrm{mmol}, 160 \mathrm{uL}), \mathrm{Fe}(\mathrm{TPP}) \mathrm{Cl}(3.5 \mathrm{mg}, 2.5 \mathrm{~mol} \%)$, and $\mathrm{K}_{2} \mathrm{CO}_{3}(0.4 \mathrm{mmol}, 2$ equiv. $)$ in DCE $\left.(0.5 \mathrm{~mL})\right)$ was added into $10 \mathrm{ml}$ electrolyte $\left(0.1 \mathrm{M} \mathrm{Bu}_{4} \mathrm{NClO}_{4}\right.$ in DCE) before the $\mathrm{CV}$ measurements in the Glove box. All $\mathrm{CV}$ experiments were referred to $\mathrm{Fc} / \mathrm{Fc}^{+}$ as internal standard under argon.

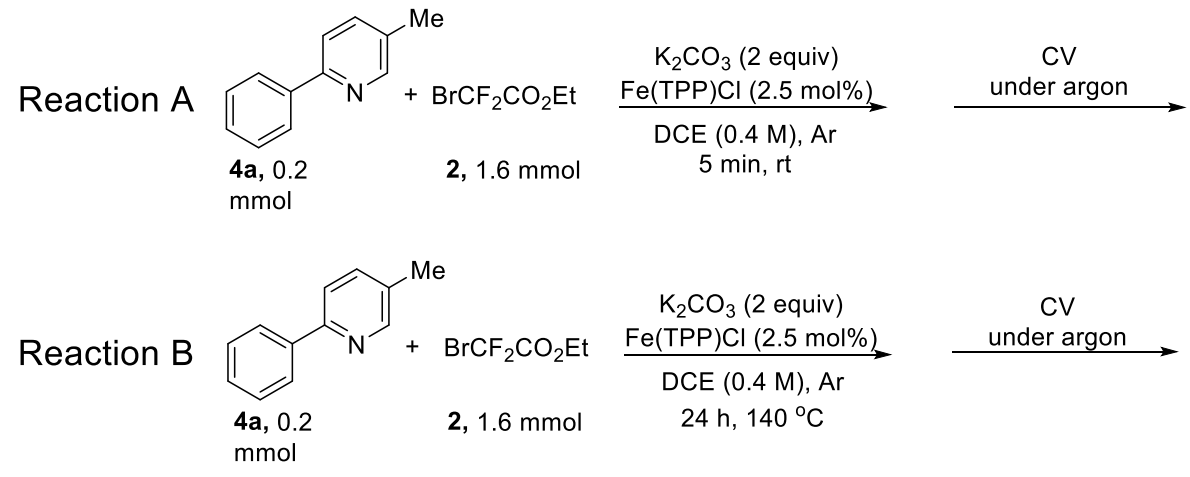


Results and Discussion:

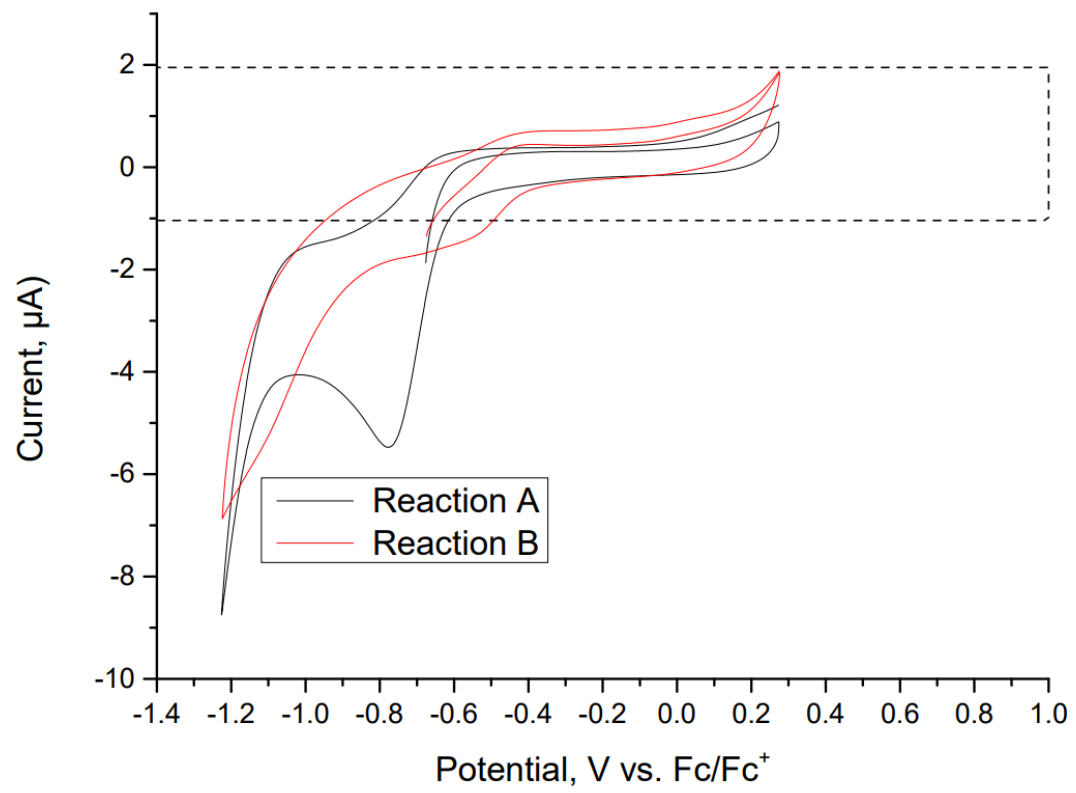

Figure S1. Cyclic voltammograms of $\mathrm{Fe}(\mathrm{TPP}) \mathrm{Cl}$ in DCE, 0.1 M TBAP.

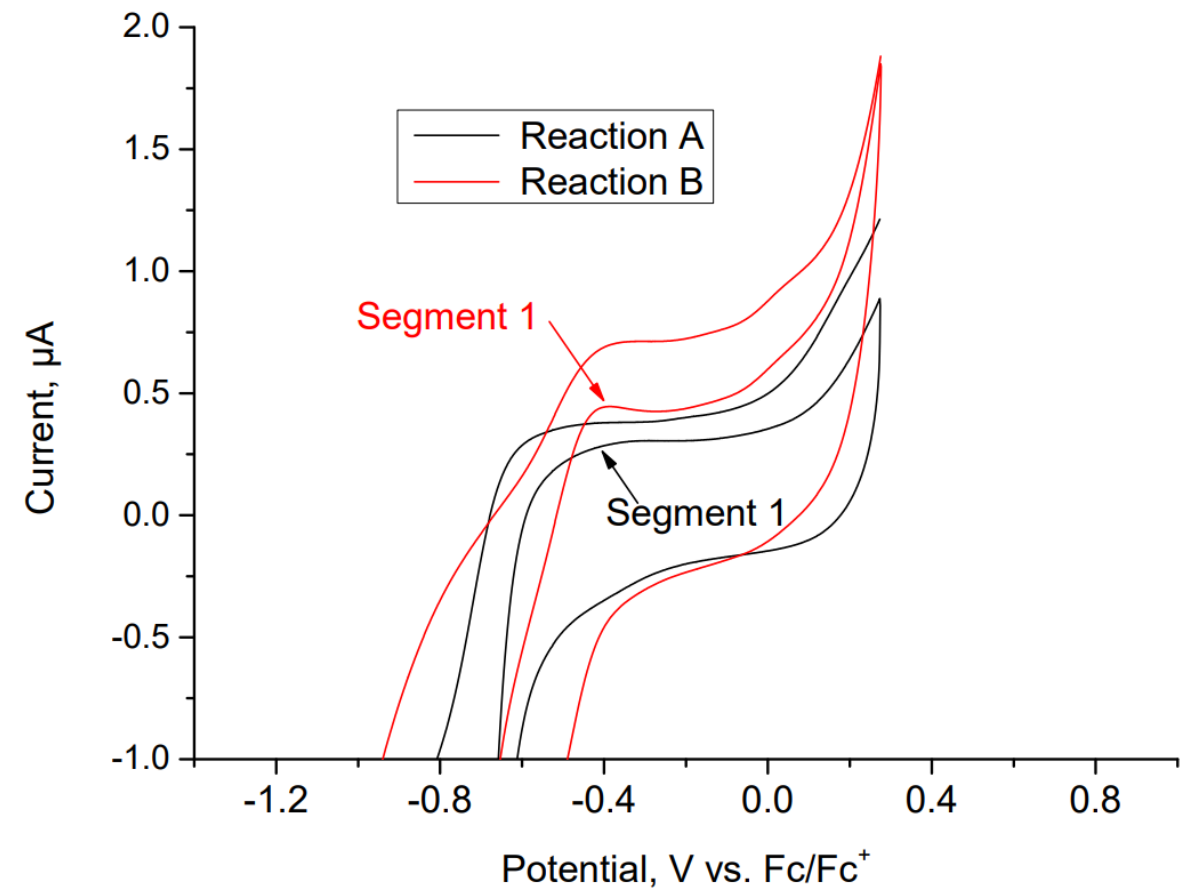

Figure S2. Local magnification of the dashed rectangle in Figure S1.

$\mathrm{E}_{1 / 2}$ (half-wave potential) of $\mathrm{Fe}(\mathrm{III}) \mathrm{TPP}-\mathrm{Fe}(\mathrm{II}) \mathrm{TPP}$ is at $-0.29 \mathrm{~V}$ vs. $\mathrm{SCE}$ in $\mathrm{CH}_{2} \mathrm{Cl}_{2}{ }^{7}$. Conversion constants between $\mathrm{Fc} / \mathrm{Fc}^{+}$and SCE electrode is $0.38 \mathrm{~V}^{8}$. So, the $\mathrm{E}_{1 / 2}$ of [ $\left.\mathrm{Fe}(\mathrm{III}) \mathrm{TPP}\right]^{+}-[\mathrm{Fe}(\mathrm{II}) \mathrm{TPP}]^{0}$ should be at $-0.67 \mathrm{~V}$ vs. $\mathrm{Fc} / \mathrm{Fc}^{+}$. Figure $\mathrm{S} 1$ shows the cyclic voltammograms of Reaction A (black line in Fig S1.) and Reaction B (red line in Fig S1.). The $\mathrm{E}_{1 / 2}$ of Reaction A is at $-0.66 \mathrm{~V} \mathrm{vs} \mathrm{Fc/Fc}$, while the $\mathrm{E}_{1 / 2}$ of Reaction $\mathrm{B}$ is at $-0.48 \mathrm{~V}$. The $\mathrm{Fe}(\mathrm{III})-\mathrm{Fe}(\mathrm{II})$ transition is highly dependent on the coordinating ability ${ }^{7}$. The $\mathrm{E}_{1 / 2}$ shift may be accounted for the stronger coordinating of $\mathbf{4 a}$ in reaction 
$\mathrm{B}$ after high temperature reaction than Reaction A. The anodic peak in the first segment of Reaction A (black line in Fig S1.) is not obvious, which may indicate that there is not any $\mathrm{Fe}(\mathrm{II})$ in Reaction A. A small anodic peak at $-0.40 \mathrm{~V}$ can be found in the first segment of Reaction B (red line in Fig S1.) which might suggest the Fe(II) was generated in Reaction B.

\section{Ultraviolet Rays}

Under different conditions, the reaction system was tested by UV. Compared with A and B, the characteristic peaks of $\mathrm{Fe}(\mathrm{TPP}) \mathrm{Cl}$ in $\mathrm{C}$ and $\mathrm{D}$ have an optical phenomenon of red shift, which indicates that there may be the formation of divalent iron in the reaction system at high temperature. It is also possible that $\mathbf{4 a}$ has coordination with $\mathrm{Fe}(\mathrm{TPP}) \mathrm{Cl}$. The test methods and results are as follows.

B<smiles>CCOC(=O)C(Br)(Br)Br</smiles>
4a, $0.2 \mathrm{mmol}$

$2,1.6 \mathrm{mmol}$

$$
\begin{gathered}
\mathrm{K}_{2} \mathrm{CO}_{3} \text { (2 equiv) } \\
\stackrel{\mathrm{Fe}(\mathrm{TPP}) \mathrm{Cl}(5 \mathrm{~mol} \%)}{\longrightarrow} \\
\underset{\mathrm{DCE}(0.4 \mathrm{M}), \mathrm{Ar}}{\mathrm{rt}} \mathrm{h}
\end{gathered}
$$
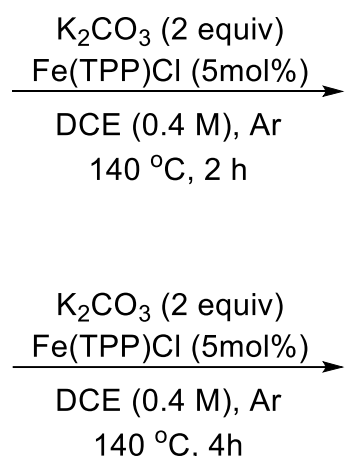

A. $\mathrm{Fe}(\mathrm{TPP}) \mathrm{Cl}(7 \mathrm{mg})$ was dissolved in DCE $(5 \mathrm{ml})$ in a beaker. The mixture $(20 \mu \mathrm{l})$ was further dissolved in $5 \mathrm{~mL}$ DCE in another beaker. The diluted mixture was further analyzed by UV.

B. A mixture of $4 \mathbf{a}\left(0.2 \mathrm{mmol}, 1.0\right.$ equiv), $\mathrm{BrCF}_{2} \mathrm{CO}_{2} \mathrm{Et}(1.2 \mathrm{mmol}, 154 \mu \mathrm{L}, 242.4 \mathrm{mg}, 6$ equiv), $\mathrm{Fe}(\mathrm{TPP}) \mathrm{Cl}(7 \mathrm{mg}, 5 \mathrm{~mol} \%), \mathrm{K}_{2} \mathrm{CO}_{3}(55.3 \mathrm{mg}, 200 \mathrm{~mol} \%)$, and DCE $(0.5 \mathrm{~mL})$ in a $15 \mathrm{~mL}$ glass vial was stirred under argon atmosphere at $25^{\circ} \mathrm{C}$ for 2 hours. The reaction mixture was further diluted and analyzed by $\mathrm{UV}$ as procedure $\mathrm{A}$.

C. A mixture of $4 \mathbf{a}\left(0.2 \mathrm{mmol}, 1.0\right.$ equiv), $\mathrm{BrCF}_{2} \mathrm{CO}_{2} \mathrm{Et}(1.2 \mathrm{mmol}, 154 \mu \mathrm{L}, 242.4 \mathrm{mg}, 6$ equiv), $\mathrm{Fe}(\mathrm{TPP}) \mathrm{Cl}(7 \mathrm{mg}, 5 \mathrm{~mol} \%), \mathrm{K}_{2} \mathrm{CO}_{3}(55.3 \mathrm{mg}, 200 \mathrm{~mol} \%)$, and DCE $(0.5 \mathrm{~mL})$ in a $15 \mathrm{~mL}$ glass vial under argon atmosphere was heated at $140^{\circ} \mathrm{C}$ for 2 hours. The reaction mixture was further diluted and analyzed by $\mathrm{UV}$ as procedure $\mathrm{A}$.

D. A mixture of $4 \mathbf{a}\left(0.2 \mathrm{mmol}, 1.0\right.$ equiv), $\mathrm{BrCF}_{2} \mathrm{CO}_{2} \mathrm{Et}(1.2 \mathrm{mmol}, 154 \mu \mathrm{L}, 242.4 \mathrm{mg}, 6$ equiv), $\mathrm{Fe}(\mathrm{TPP}) \mathrm{Cl}(7 \mathrm{mg}, 5 \mathrm{~mol} \%), \mathrm{K}_{2} \mathrm{CO}_{3}(55.3 \mathrm{mg}, 200 \mathrm{~mol} \%)$, and DCE $(0.5 \mathrm{~mL})$ in a $15 \mathrm{~mL}$ glass vial under argon atmosphere was heated at $140^{\circ} \mathrm{C}$ for 4 hours. The reaction mixture was further diluted and analyzed by $\mathrm{UV}$ as procedure $\mathrm{A}$. 

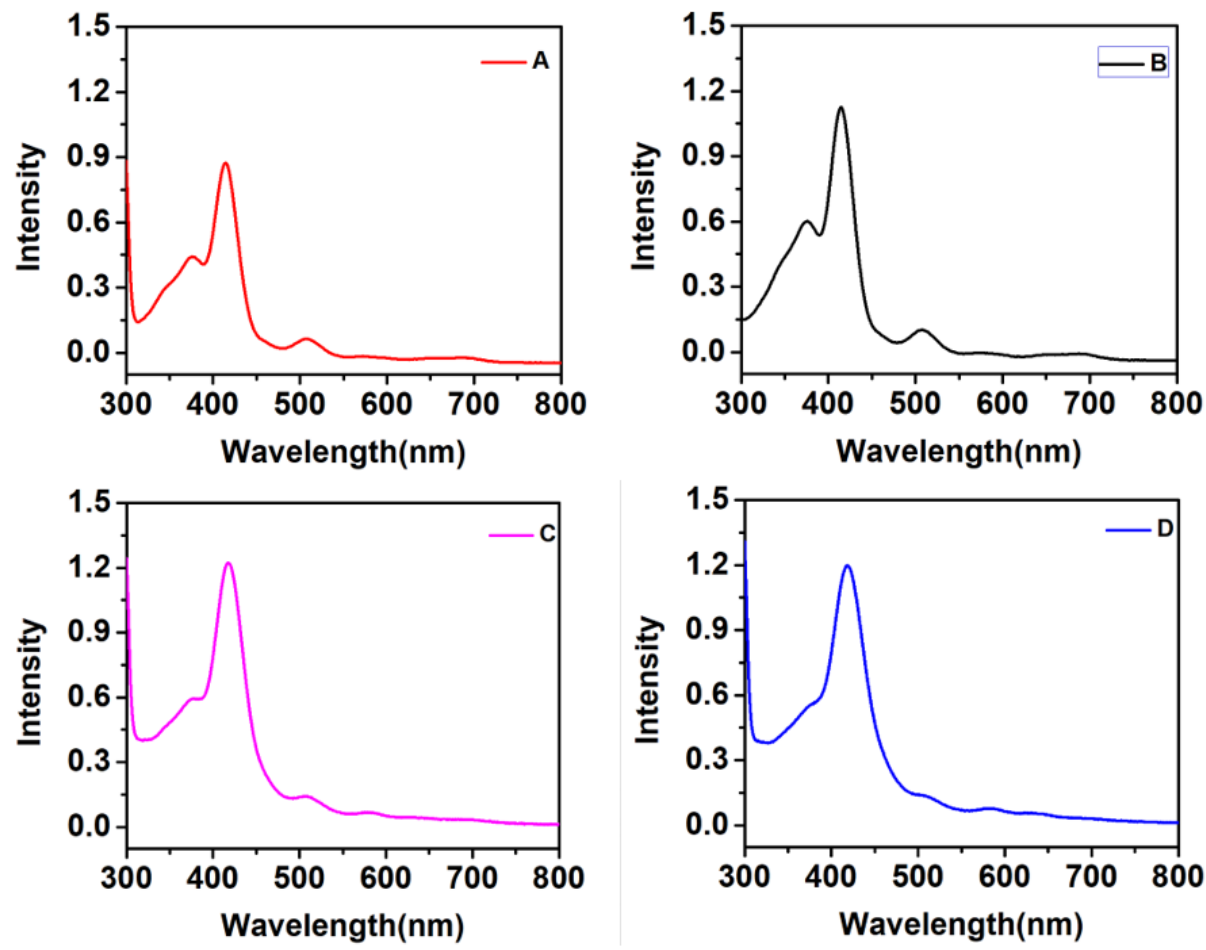

Figure S3. Optical absorption of A, B, C and D in DCE.

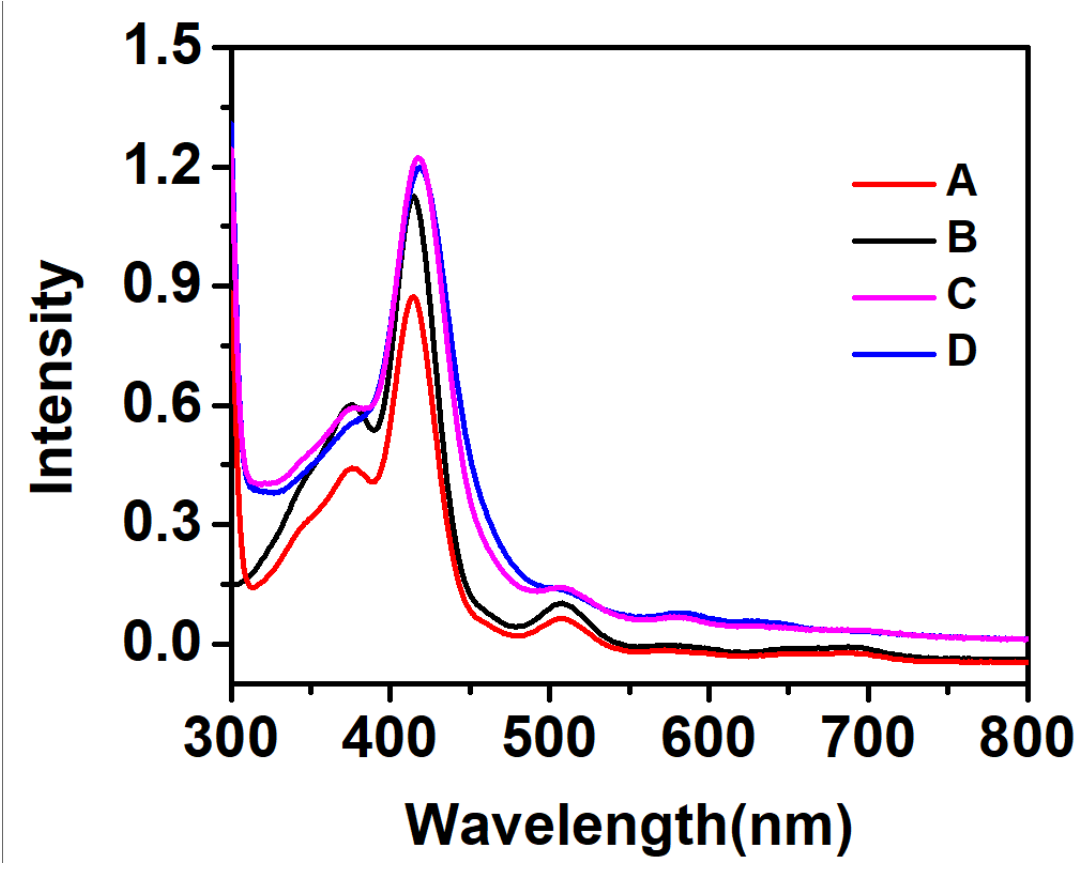

Figure S4. Superposition of optical absorption of A, B, C and D in DCE. 


\section{EPR Studies}

In order to obtain the information of intermediate-II, we carried out the following EPR experiments. Unfortunately, we can only detect the signal peak of capturing ethyl difluoroacetate radical.

A. Reaction of $\mathbf{2}$ in the presence of PBN.

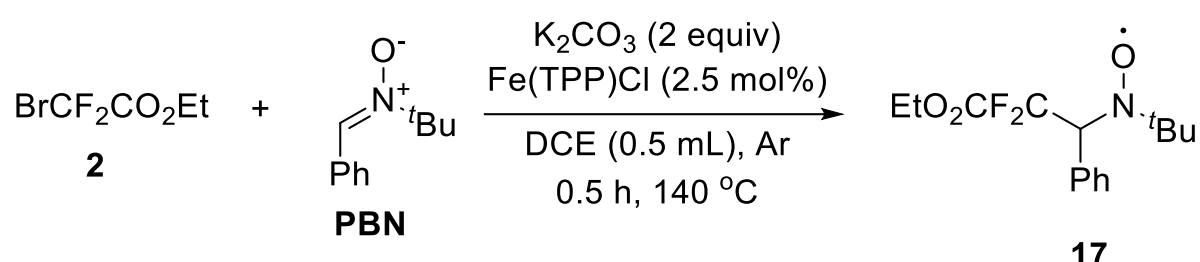

Procedure: A mixture of $\mathrm{BrCF}_{2} \mathrm{CO}_{2} \mathrm{Et}(0.2 \mathrm{mmol}), \mathbf{P B N}(0.3 \mathrm{mmol}), \mathrm{Fe}(\mathrm{TPP}) \mathrm{Cl}(3.5 \mathrm{mg}), \mathrm{K}_{2} \mathrm{CO}_{3}$ $(55.3 \mathrm{mg}, 200 \mathrm{~mol} \%)$, and DCE $(0.5 \mathrm{~mL})$ in a $15 \mathrm{~mL}$ glass vial under argon atmosphere was heated at $140{ }^{\circ} \mathrm{C}$ for 0.5 hours. the resulting mixture was analyzed by EPR. The EPR showed an e.p.r. spectrum of nitroxides $17(\mathrm{~g}=2.005)$. Based on the previous report ${ }^{9}$, we speculated the product 17 was formed. This result is consist with proposed catalytic cycle that the $\mathrm{Fe}(\mathrm{TPP}) \mathrm{Cl}$ plays an important role in generating the $\cdot \mathrm{CF}_{2} \mathrm{CO}_{2}$ Et free radical.

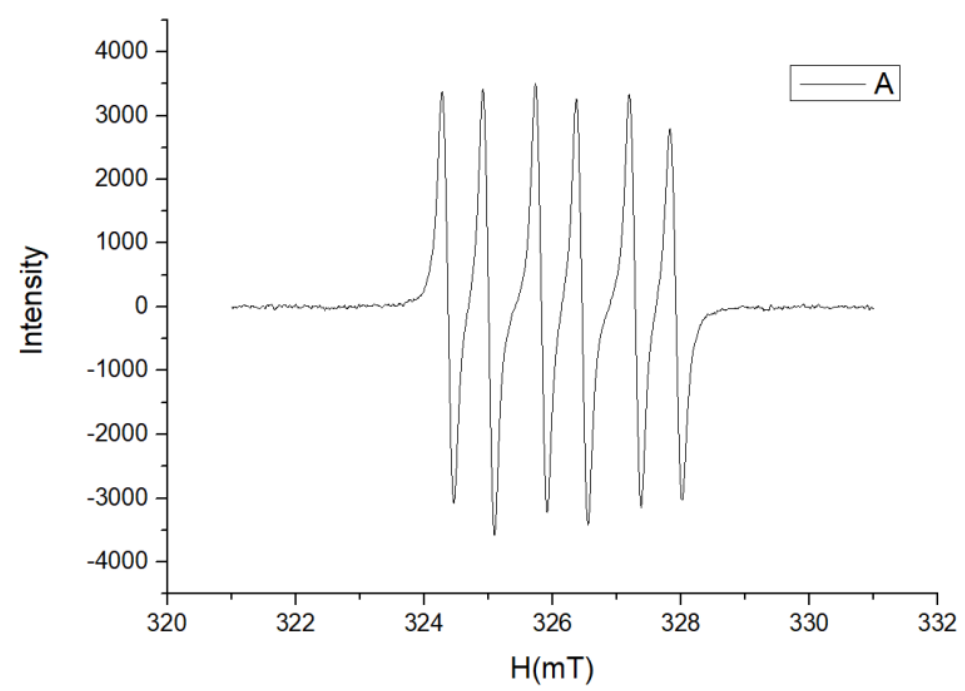

Figure S5. The EPR spectrum of a mixture of A.

B. Reaction of $\mathbf{2}$ and $\mathbf{4 a}$ in the presence of $\mathrm{PBN}$.

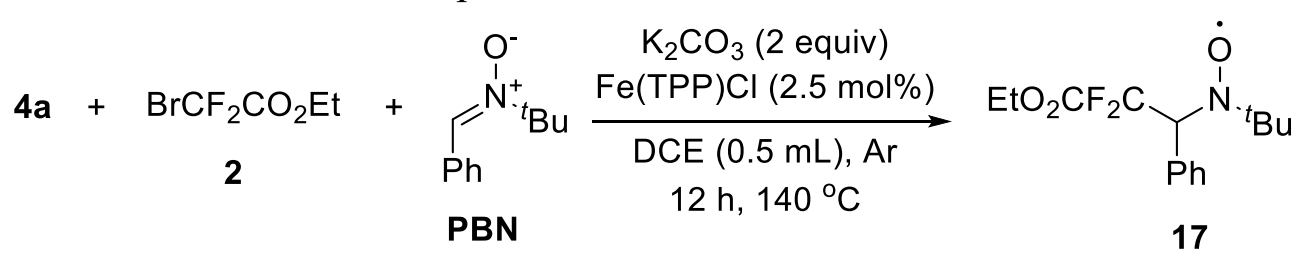

Procedure: A mixture of $4 \mathrm{a}(0.2 \mathrm{mmol}), \mathrm{BrCF}_{2} \mathrm{CO}_{2} \mathrm{Et}(0.4 \mathrm{mmol}), \mathbf{P B N}(0.5 \mathrm{mmol}), \mathrm{Fe}(\mathrm{TPP}) \mathrm{Cl}$ (3.5 mg, $2.5 \mathrm{~mol} \%), \mathrm{K}_{2} \mathrm{CO}_{3}(55.3 \mathrm{mg}, 200 \mathrm{~mol} \%$ ), and DCE $(0.5 \mathrm{~mL})$ in a $15 \mathrm{~mL}$ glass vial under 
argon atmosphere was heated at $140{ }^{\circ} \mathrm{C}$ for 12 hours. The resulting mixture was analyzed by EPR. The EPR showed an e.p.r. spectrum of nitroxides $17(\mathrm{~g}=2.005)$, which suggested the $\cdot \mathrm{CF}_{2} \mathrm{CO}_{2} \mathrm{Et}$ free radical was formed during the catalytic cycle.

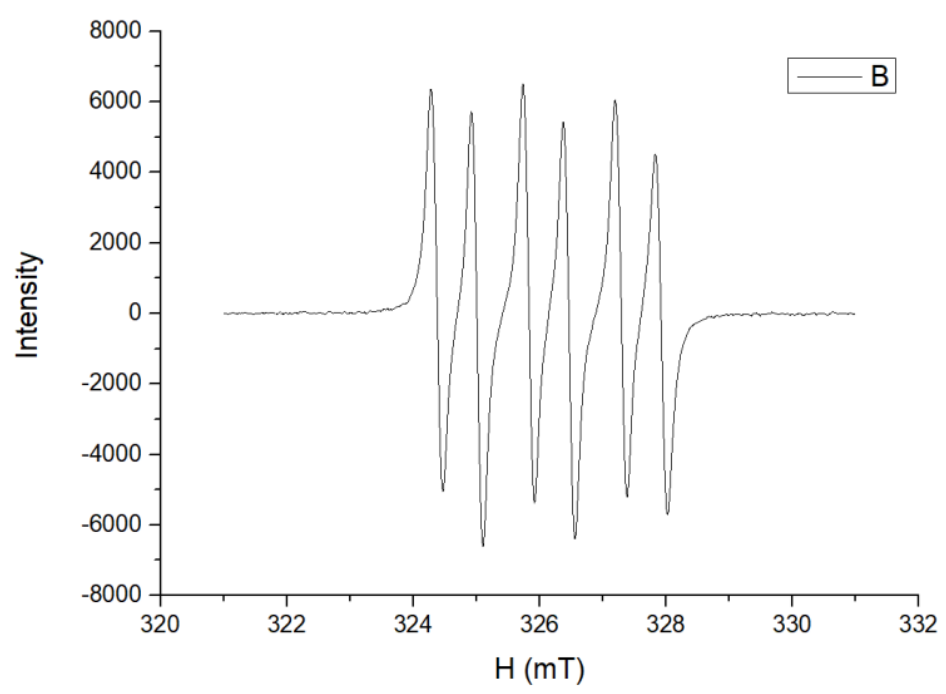

Figure S6. The EPR spectrum of a mixture of B.

C. Reaction of $\mathbf{2}$ in the presence of tert-Nitrosobutanedimer $\mathbf{1 8 .}$

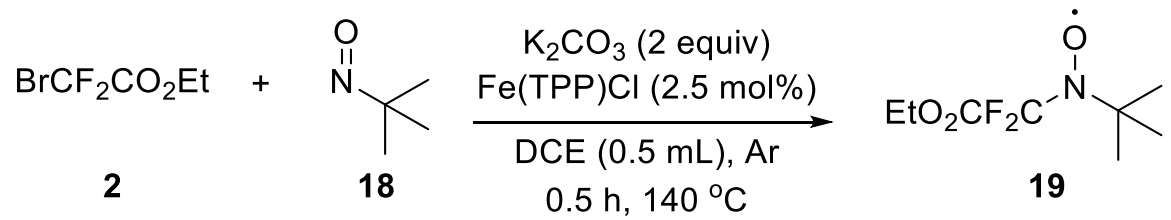

Procedure: A mixture of $\mathrm{BrCF}_{2} \mathrm{CO}_{2} \mathrm{Et}(0.2 \mathrm{mmol})$, tert-Nitrosobutanedimer 18 (0.3 mmol), $\mathrm{Fe}(\mathrm{TPP}) \mathrm{Cl}$ (3.5 mg, $2.5 \mathrm{~mol} \%), \mathrm{K}_{2} \mathrm{CO}_{3}(55.3 \mathrm{mg}, 200 \mathrm{~mol} \%$ ), and DCE $(0.5 \mathrm{~mL})$ in a $15 \mathrm{~mL}$ glass vial under argon atmosphere was heated at $140{ }^{\circ} \mathrm{C}$ for 0.5 hours. The resulting mixture was analyzed by EPR. The EPR showed an e.p.r. spectrum of nitroxides $19(\mathrm{~g}=2.005)$.

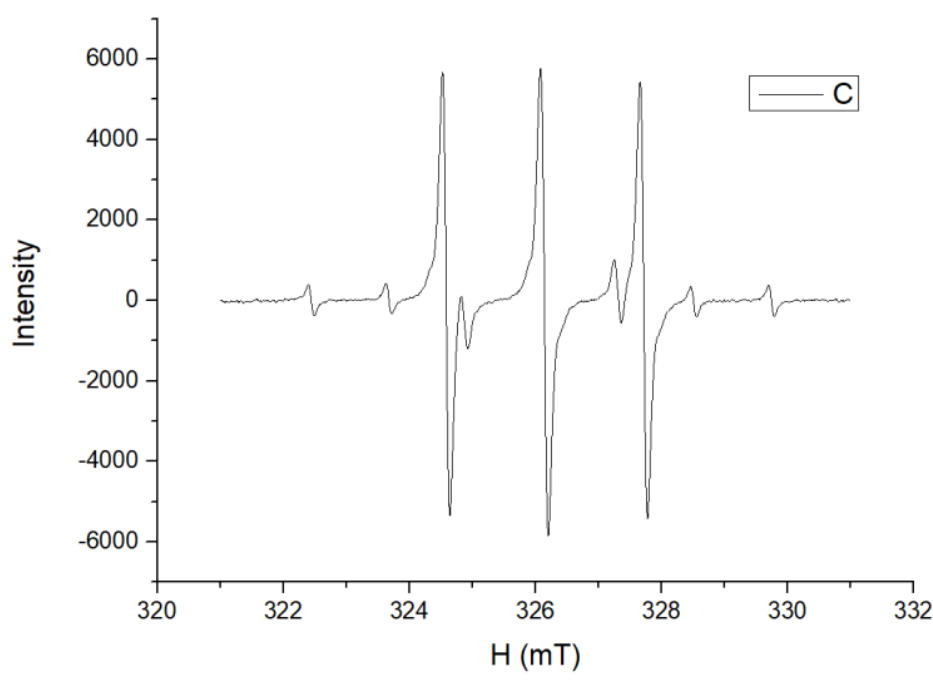


Figure S7. The EPR spectrum of a mixture of C.

D. Reaction of $\mathbf{2}$ and $\mathbf{4 a}$ in the presence of tert-Nitrosobutanedimer $\mathbf{1 8 .}$

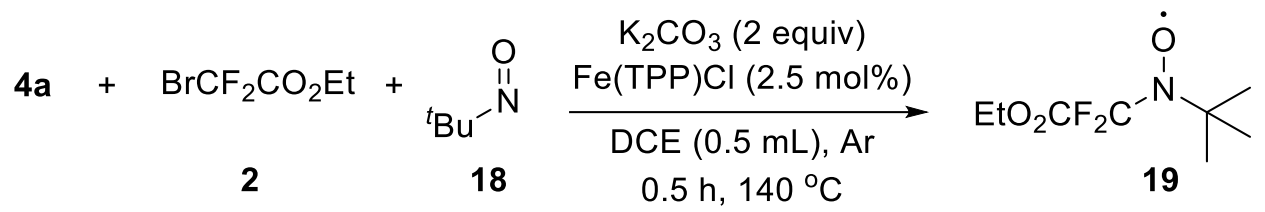

Procedure: A mixture of $\mathbf{4 a}(0.2 \mathrm{mmol}), \mathbf{2}(0.4 \mathrm{mmol})$, tert-Nitrosobutanedimer $18(0.5 \mathrm{mmol})$, $\mathrm{Fe}(\mathrm{Tpp}) \mathrm{Cl}$ (3.5 mg, $2.5 \mathrm{~mol} \%), \mathrm{K}_{2} \mathrm{CO}_{3}(55.3 \mathrm{mg}, 200 \mathrm{~mol} \%)$, and DCE $(0.5 \mathrm{~mL})$ in a $15 \mathrm{~mL}$ glass vial under argon atmosphere was heated at $140{ }^{\circ} \mathrm{C}$ for 12 hours. The resulting mixture was analyzed by EPR. The EPR showed an e.p.r. spectrum of nitroxides 19 ( $\mathrm{g}=2.005)$.

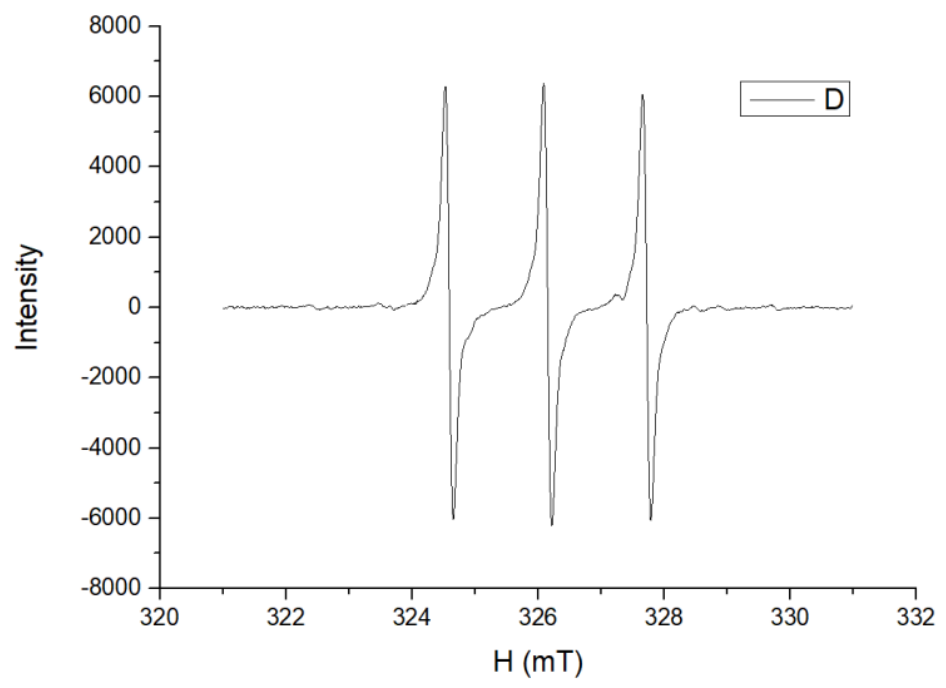

Figure S8. The EPR spectrum of a mixture of D.

E. Reaction of $\mathbf{2}$ and $\mathbf{4 a}$ under standard conditions.

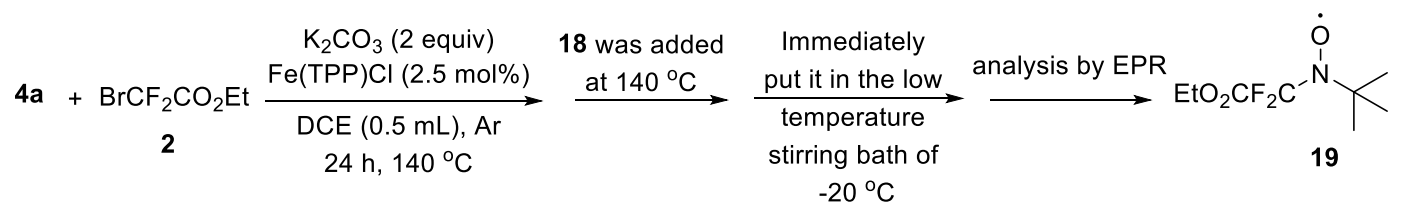

Procedure: A mixture of $4 \mathbf{a}(0.2 \mathrm{mmol}), 2$ (1.2 mmol), $\mathrm{Fe}(\mathrm{TPP}) \mathrm{Cl}(3.5 \mathrm{mg}, 2.5 \mathrm{~mol} \%), \mathrm{K}_{2} \mathrm{CO}_{3}(55.3$ $\mathrm{mg}, 200 \mathrm{~mol} \%)$, and DCE $(0.5 \mathrm{~mL})$ in a $15 \mathrm{~mL}$ glass vial sealed under argon atmosphere was heated at $140^{\circ} \mathrm{C}$ for 24 hours. Then tert-Nitrosobutanedimer $18(0.5 \mathrm{mmol})$ in DCE $(0.25 \mathrm{ml})$ was fed into the glass vial through a syringe. Then, the reaction tube was immediately placed in the low temperature stirring bath of $-20^{\circ} \mathrm{C}$. The resulting mixture was analyzed by EPR. The EPR showed an e.p.r. spectrum of nitroxides $19(\mathrm{~g}=2.005)$. 


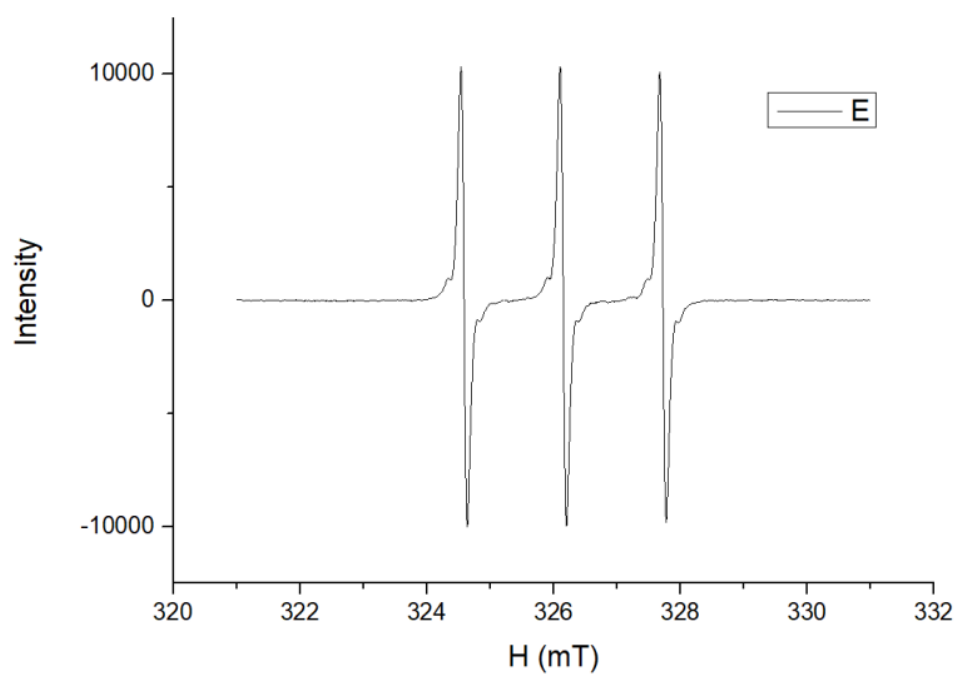

Figure S9. The EPR spectrum of a mixture of E.

After these EPR experiments, it suggested the ${ }^{-} \mathrm{CF}_{2} \mathrm{CO}_{2} \mathrm{Et}$ was generated in the reaction system. However, we can't trap the intermediate II by the EPR experiments. It might be because the intermediate II is highly unstable, and would be directly transformed into intermediate III, when it was generated in the catalytic cycle.

\section{The HRMS of 20.}<smiles>COc1ccc(-c2ccccc2)nc1</smiles>

$4 \mathbf{v}, 0.1 \mathrm{mmol}$
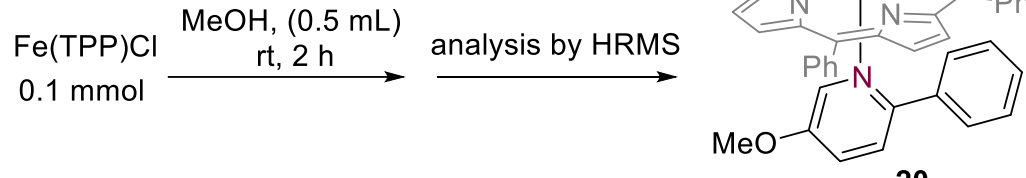

20

HRMS Calcd for $\mathrm{C}_{56} \mathrm{H}_{39} \mathrm{FeN}_{5} \mathrm{O}^{3+}[\mathrm{M}]: 853.2488$

Found: 853.2445.

When the substrate $4 \mathbf{v}(0.1 \mathrm{mmol})$ was treated with $\mathrm{Fe}(\mathrm{TPP}) \mathrm{Cl}(0.1 \mathrm{mmol})$ in methanol at room temperature for $2 \mathrm{hrs}$. The mixture was further analyzed by HRMS. To our great delight, a molecular weight 853.2445 was obtained (Figure S10), which suggested the key intermediate I may be formed in the reaction. It also suggested the $\mathrm{Fe}-\mathrm{N}$ coordination may be formed in the catalytic cycle. 


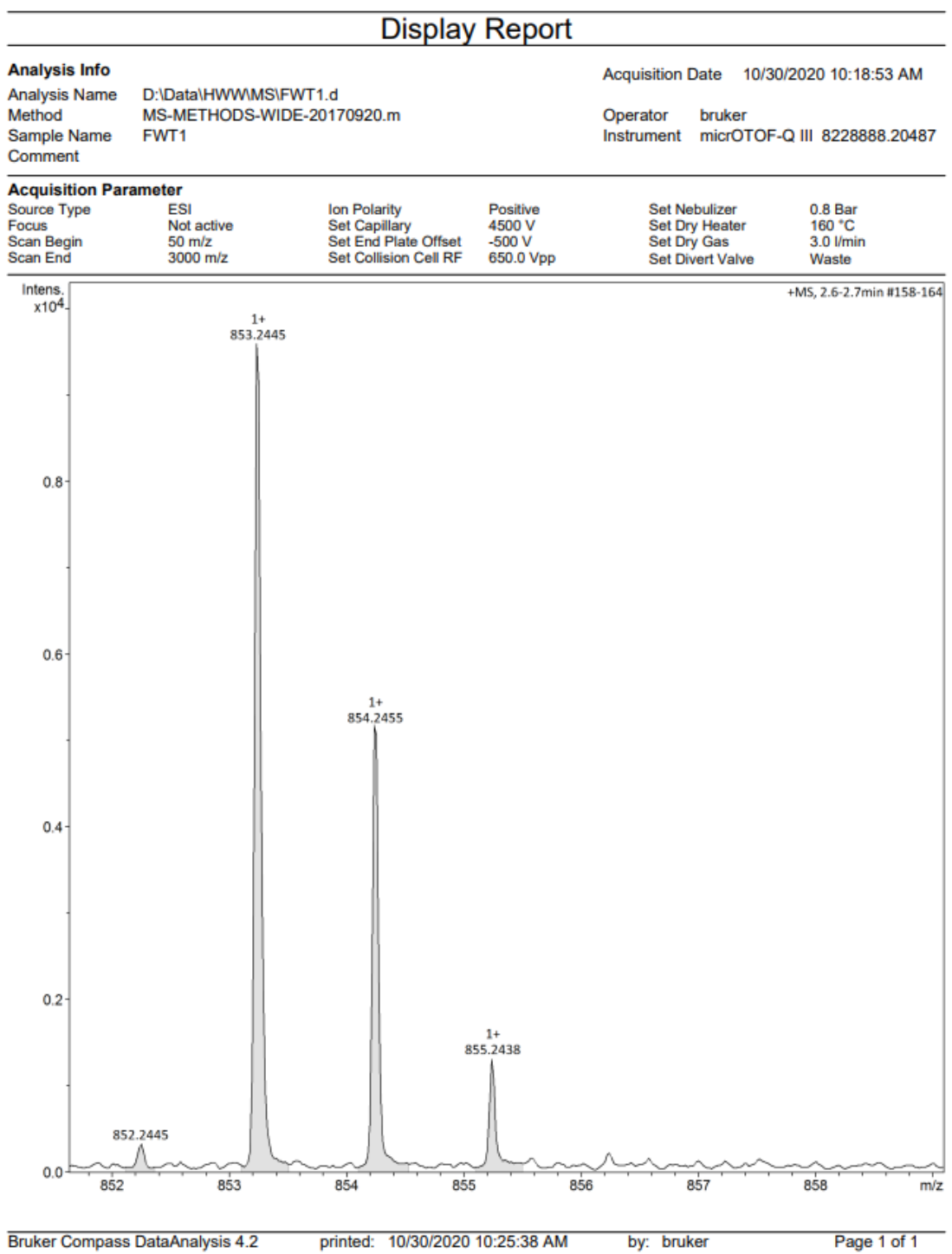

Figure S10. The HRMS of $\mathbf{2 0}$

\section{References}

1. Park, J.; Chang, S. Comparative Catalytic Activity of Group 9 [Cp*MIII] Complexes: CobaltCatalyzed C-H Amidation of Arenes with Dioxazolones as Amidating Reagents. Angew. Chem. Int. Ed. 2015, 54, 14103-14107.

2. Nishii, Y.; Bachon, A.-K.; Moon, S.; Bolm, C.; , Miura, M. Rhodium-catalyzed Synthesis of 1(Acylamino)isoquinolines through Direct Annulative Coupling of 3-Aryl-1,2,4-oxadiazoles with Alkynes. Chem. Lett. 2017, 46, 1347-1349.

3. Movassagh, B,; Talebsereshki, F. Mild and Efficient One-Pot Synthesis of 3,5-Disubstituted 1,2,4-Oxadiazoles from Nitriles Mediated by $\mathrm{K}_{3} \mathrm{PO}_{4}$. Synth. Commun. 2014, 44, 188-194.

4. Jursic, B. S.; Zdravkovskif, Z. Preparation of 5-substituted 2-methyl-1,3,4-oxadiazoles from 5substituted tetrazoles and acetic anhydride. Synth. Commun. 1994, 24, 1575-1582.

5. Chen, J.; Huang, T.; Gong, X.; Yu, Z.-J.; Shi, Y.; Yan, Y. -H.; Zheng, Y.; Liu, X.; Li, G.-B.; Wu, Y. Ruthenium-Catalyzed meta-Selective $\mathrm{C}-\mathrm{H}$ Nitration of Biologically Important 
Aryltetrazoles. Adv. Synth. Catal. 2020, 362, 2984-2989.

6. (a) Jiang, Z.; Wan, W.; Li, H.; Yuan, S.; Zhao, H.; Wong, P. K. A Hierarchical Z-Scheme a $-\mathrm{Fe}_{2} \mathrm{O}_{3} /$ g$\mathrm{C}_{3} \mathrm{~N}_{4}$ Hybrid for Enhanced Photocatalytic $\mathrm{CO}_{2}$ Reduction. Adv. Mater. 2018, 30, 1706108. (b) She, X.; Wu, J.; Xu, H.; Zhong, J.; Wang, Y.; Song, Y.; Nie, K.; Liu, Y.; Yang, Y.; Rodrigues, M.-T. F.; Vajtai, R.; Lou, J.; Du, D.; Li, H.; Ajayan, P. M. Adv. Energy Mater. 2017, 1700025.

7. Kadish, K. M.; Morrison, M. M.; Constant, L. A.; Dickens, L.; Davis, D. G. A study of solvent and substituent effects on the redox potentials and electron-transfer rate constants of substituted iron meso-tetraphenylporphyrins. J. Am. Chem. Soc. 1976, 98, 8387-8390.

8. Brady, F. J.; Cardin, C. J.; Cardin, D. J.; Wilcock, D. J. Steric stabilisation of the P-P bond in a bulky tetraorganodiphosphine: synthesis, characterisation and X-ray structure determination of tetrakis(2,4,6-triisopropylphenyl)diphosphine. Inorg. Chim. Acta, 2000, 298, 97-102.

9. Zhou, M.; Zhao, H.-Y.; Zhang, S.; Zhang, Y.; Zhang, X. Nickel-Catalyzed Four-Component Carbocarbonylation of Alkenes under 1 atm of CO. J. Am. Chem. Soc. 2020, 142, 18191-18199. 
NMR spectra

${ }^{1} \mathrm{H}$ NMR (400 MHz, $\left.\mathrm{CDCl}_{3}\right)$ of $1 \mathrm{~b}$

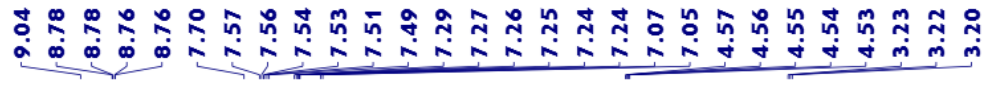

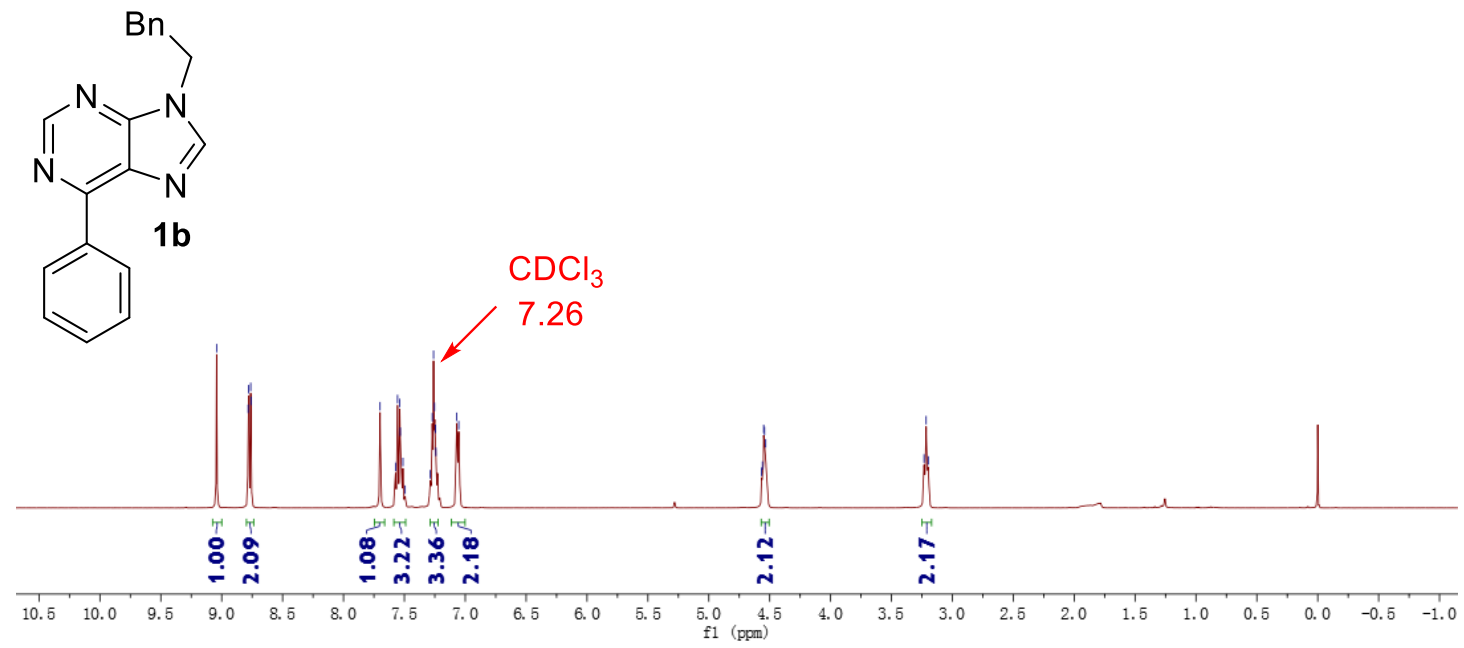

${ }^{13} \mathrm{C}$ NMR (100 MHz, $\left.\mathrm{CDCl}_{3}\right)$ of $1 \mathrm{~b}$

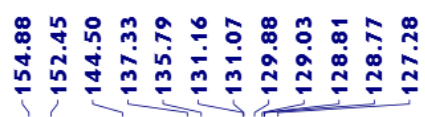

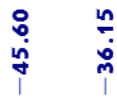

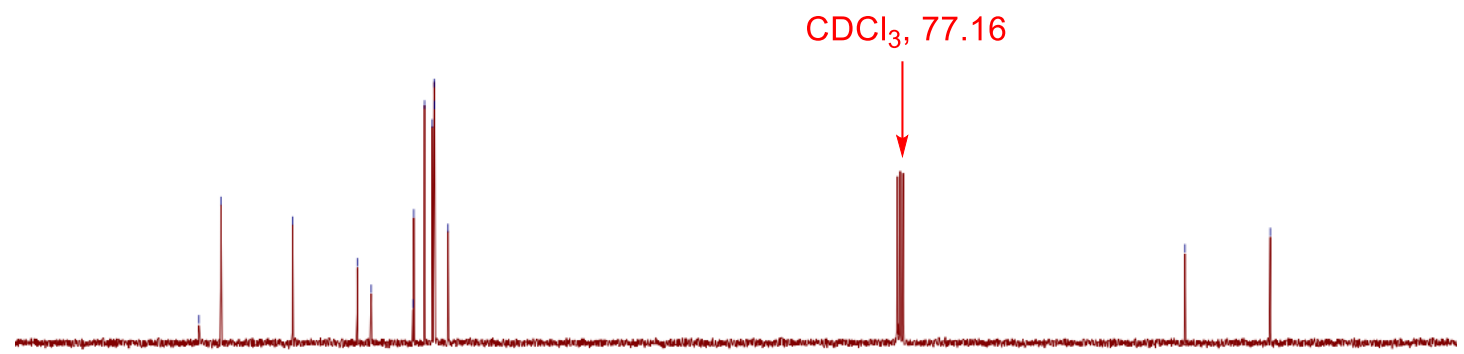

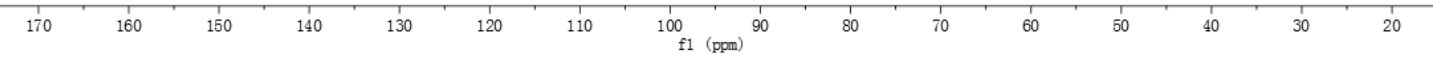


${ }^{1} \mathrm{H}$ NMR (400 MHz, $\left.\mathrm{CDCl}_{3}\right)$ of $1 \mathrm{~g}$

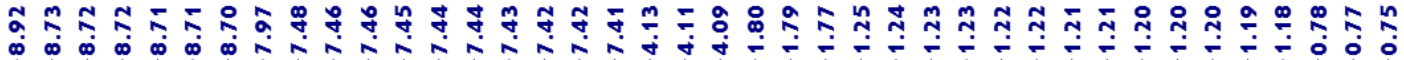

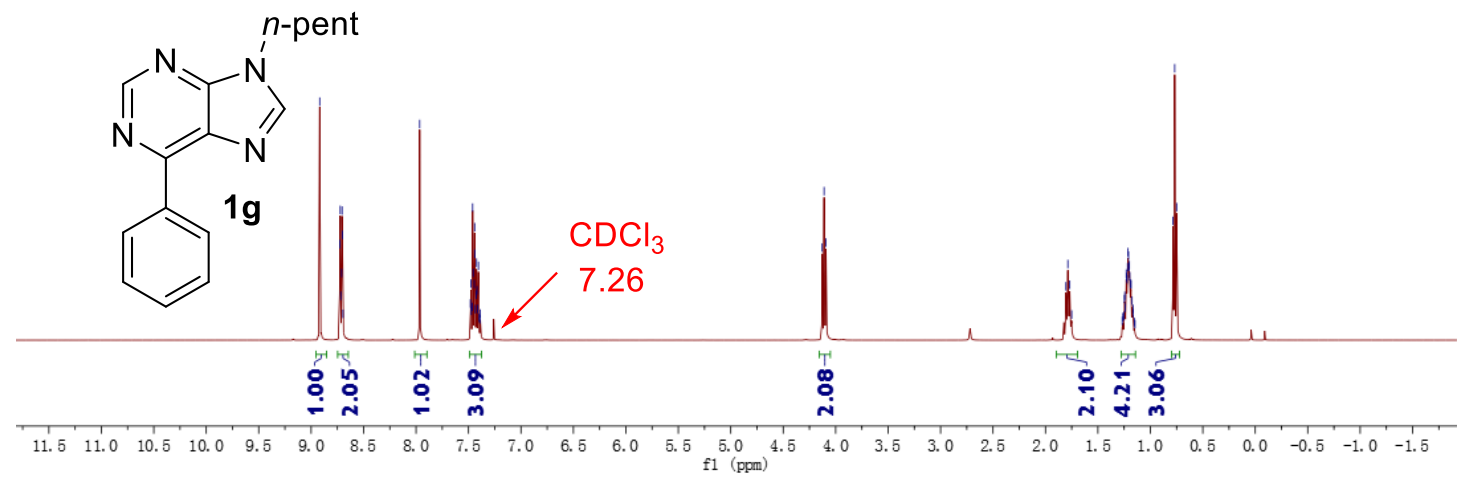

${ }^{13} \mathrm{C}$ NMR (100 MHz, CDCl 3$)$ of $1 \mathrm{~g}$

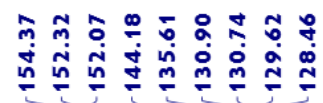

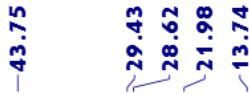

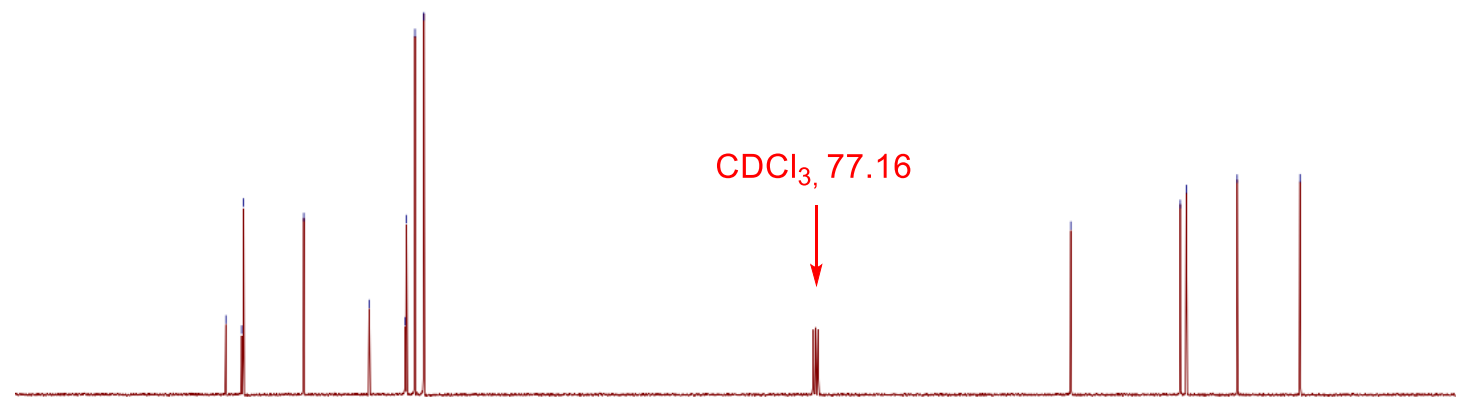

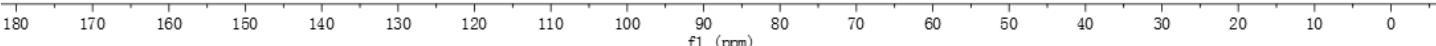


${ }^{1} \mathrm{H}$ NMR (400 MHz, $\left.\mathrm{CDCl}_{3}\right)$ of $1 \mathrm{~h}$

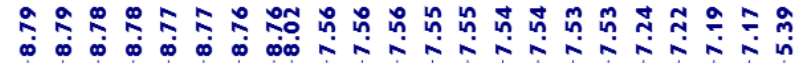

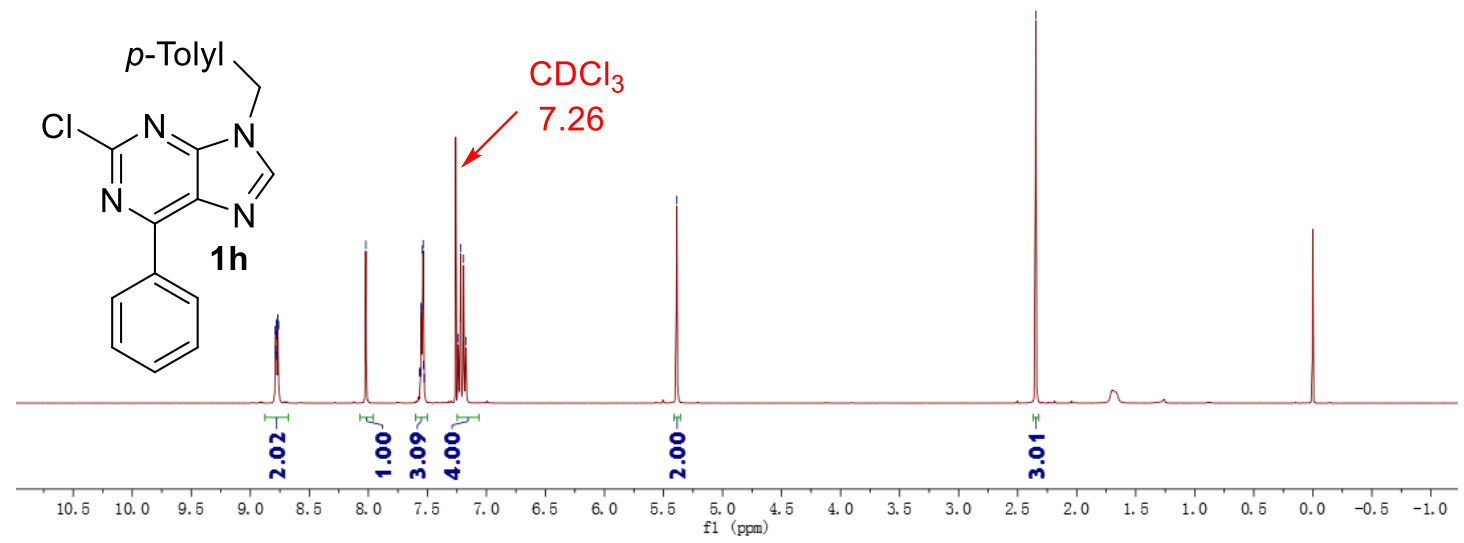

${ }^{13} \mathrm{C}$ NMR (100 MHz, CDCl 3$)$ of $1 \mathrm{~h}$

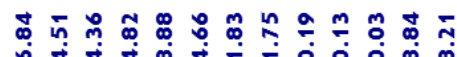

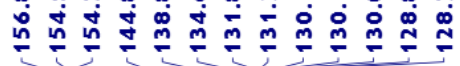

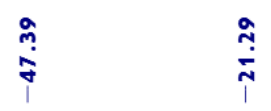

$\mathrm{CDCl}_{3}, 77.16$
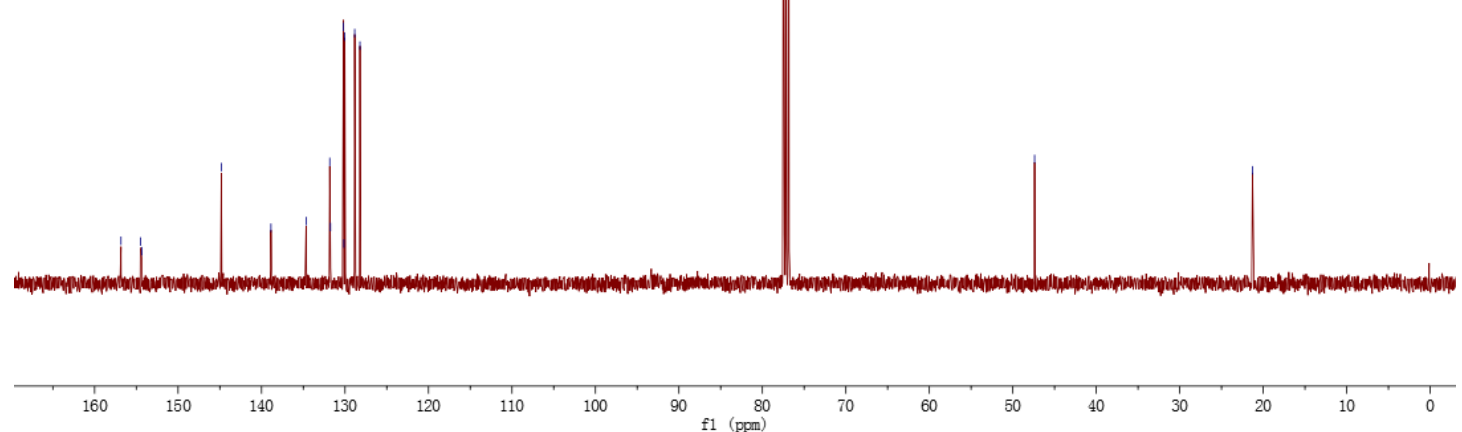
${ }^{1} \mathrm{H}$ NMR (400 MHz, $\left.\mathrm{CDCl}_{3}\right)$ of $4 \mathrm{~d}$

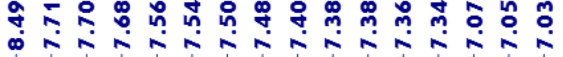

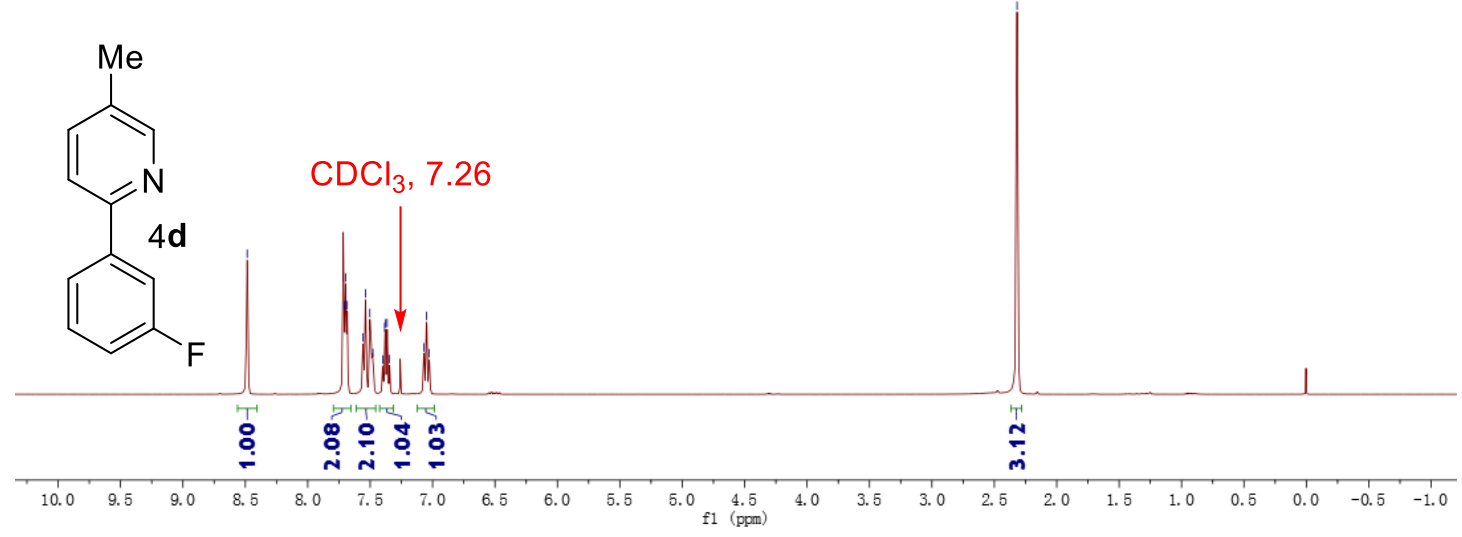

${ }^{19}$ F NMR (376 MHz, CDCl $)$ of 4d 
${ }^{13} \mathrm{C}$ NMR (100 $\left.\mathrm{MHz}, \mathrm{CDCl}_{3}\right)$ of $4 \mathrm{~d}$

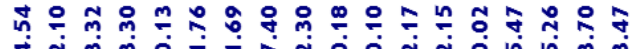

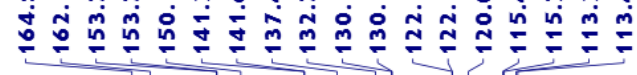
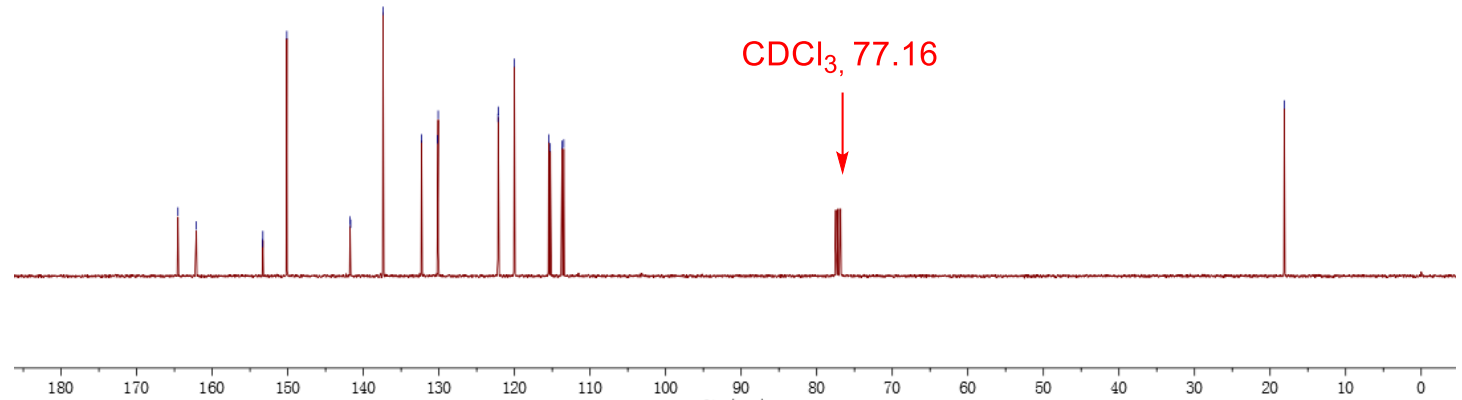

${ }^{1} \mathrm{H}$ NMR (400 MHz, $\mathrm{CDCl}_{3}$ ) $4 \mathrm{f}$

藏

$\stackrel{i}{i}$

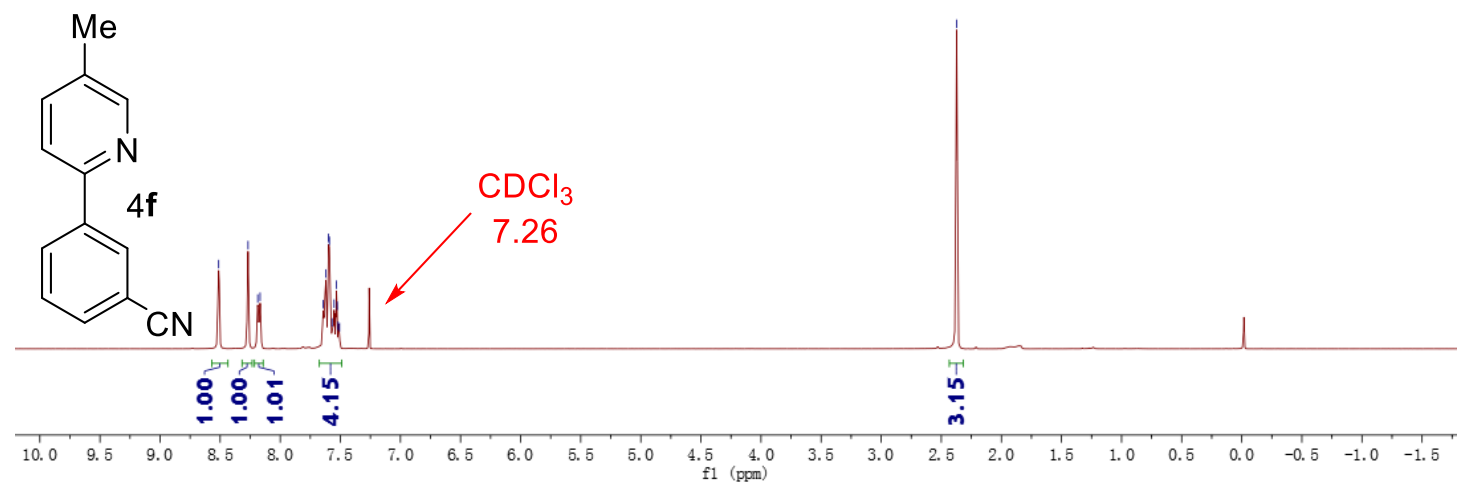


${ }^{13} \mathrm{C}$ NMR (100 $\left.\mathrm{MHz}, \mathrm{CDCl}_{3}\right)$ of $4 \mathrm{f}$

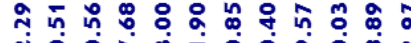

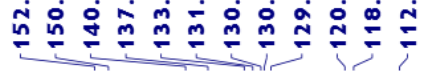

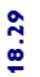
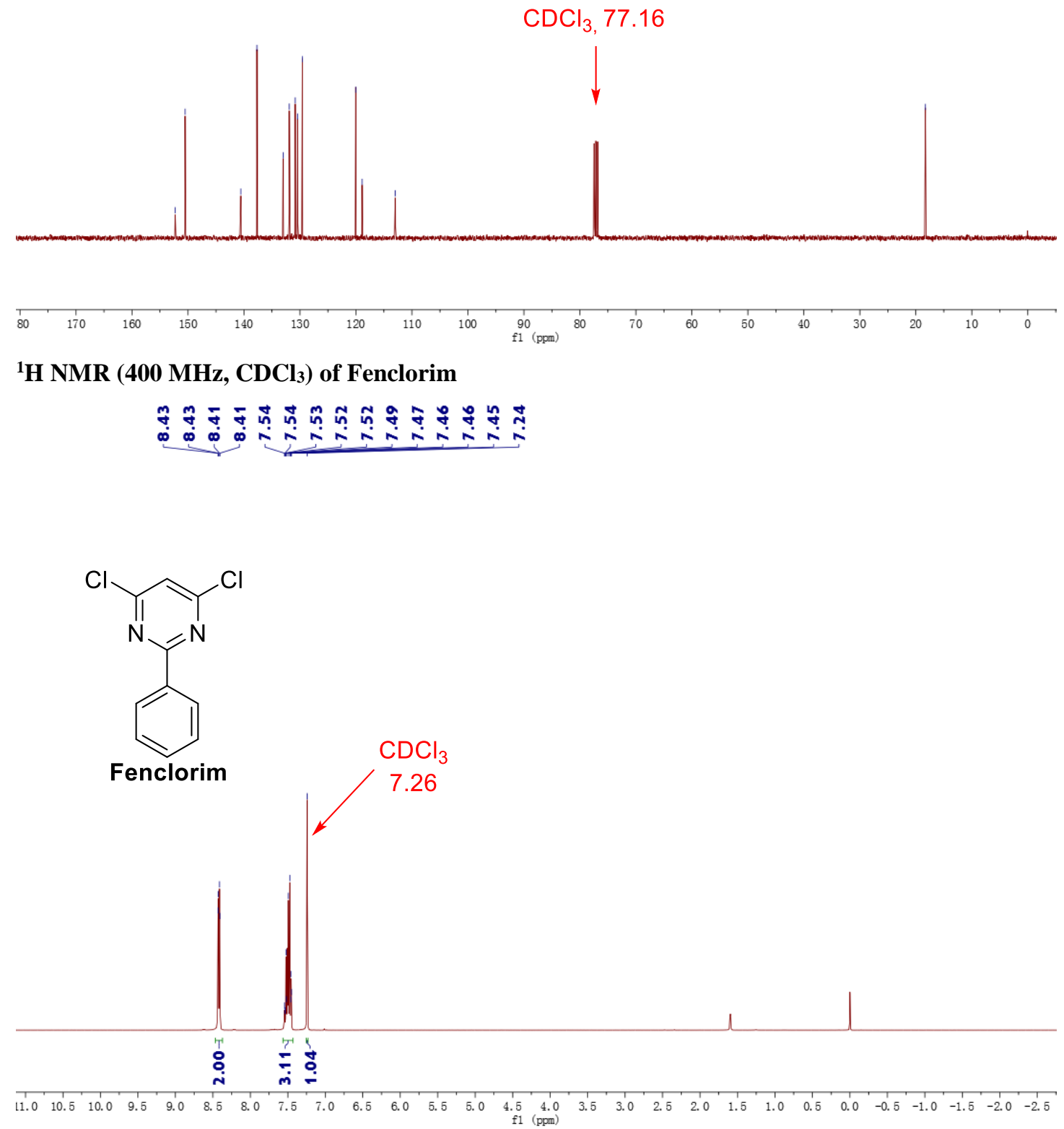
${ }^{13} \mathrm{C}$ NMR (100 MHz, $\left.\mathrm{CDCl}_{3}\right)$ of Fenclorim

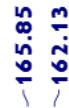

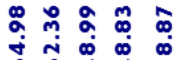

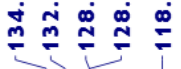

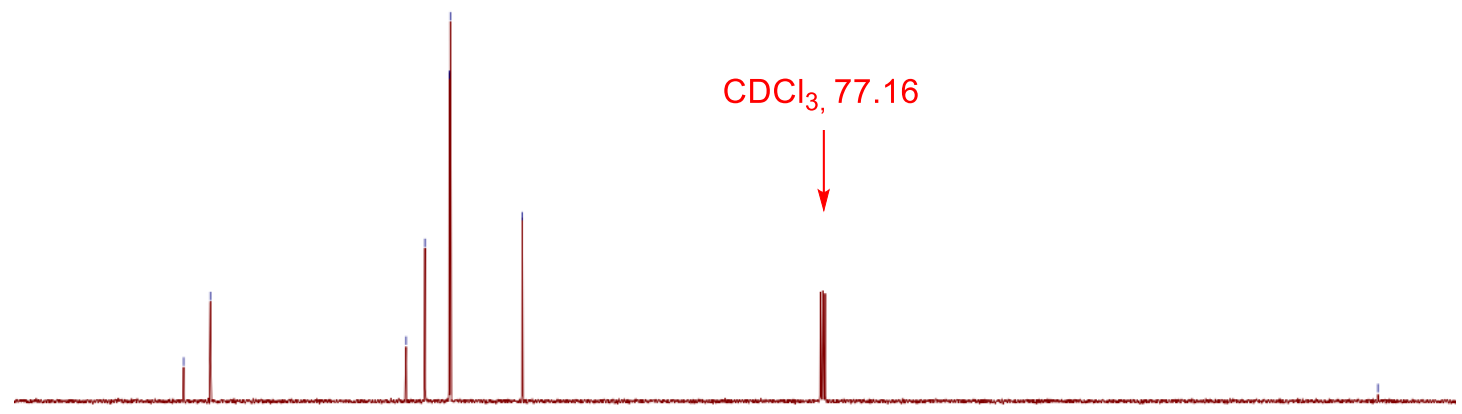

$\begin{array}{llllll}180 & 170 & 160 & 150 & 140 & 130\end{array}$

${ }^{1} \mathrm{H}$ NMR (400 MHz, $\left.\mathrm{CDCl}_{3}\right)$ of $4 \mathrm{k}$

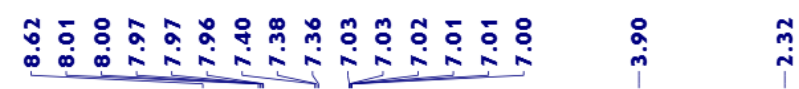

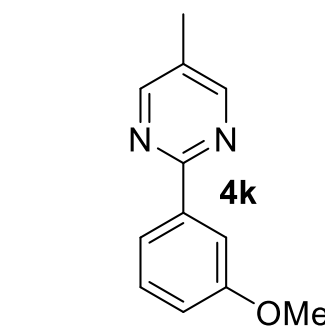

$\mathrm{CDCl}_{3}, 7.26$

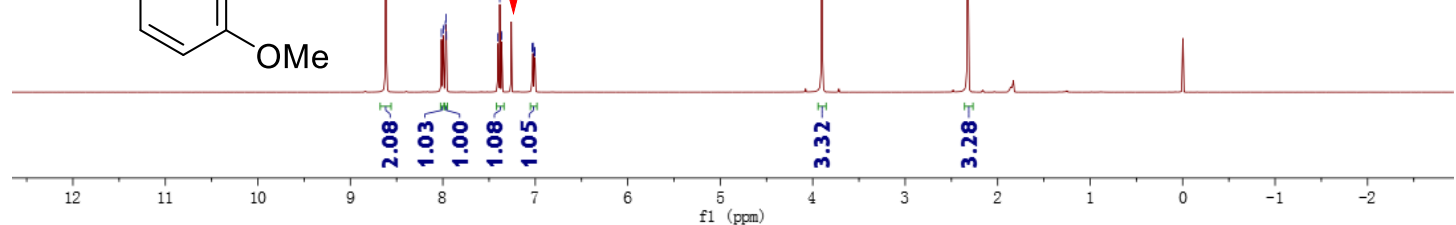


${ }^{13} \mathrm{C}$ NMR (100 $\left.\mathrm{MHz}, \mathrm{CDCl}_{3}\right)$ of $4 \mathrm{k}$

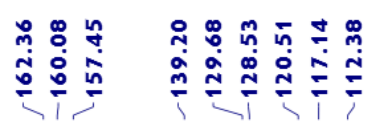

in

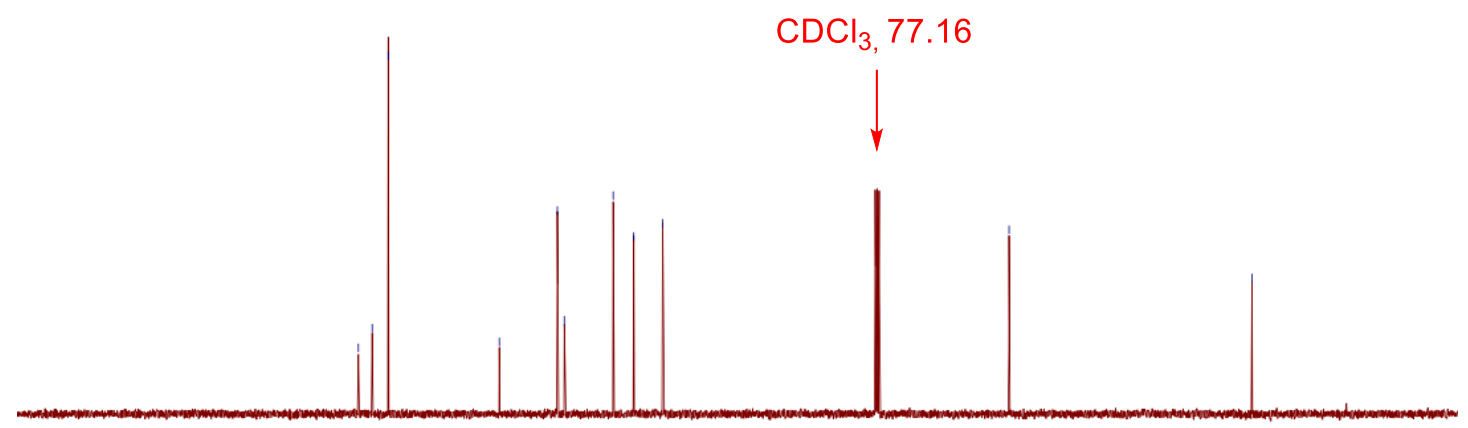

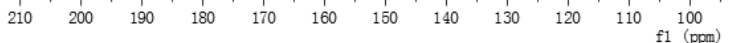

${ }^{1} \mathrm{H}$ NMR (400 $\left.\mathrm{MHz}, \mathrm{CDCl}_{3}\right)$ of $4 \mathrm{I}$

ঢீ

$\stackrel{\sim}{m}$

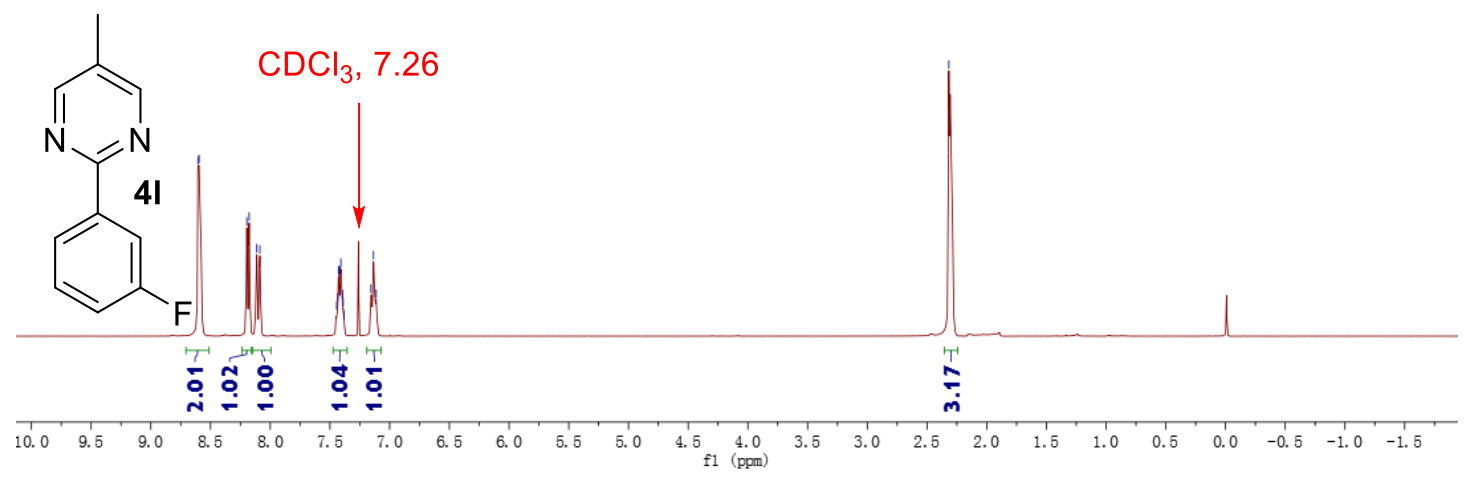


${ }^{19}$ F NMR (376 MHz, CDCl 3$)$ of 41

$$
\stackrel{n}{\mathfrak{m}}
$$

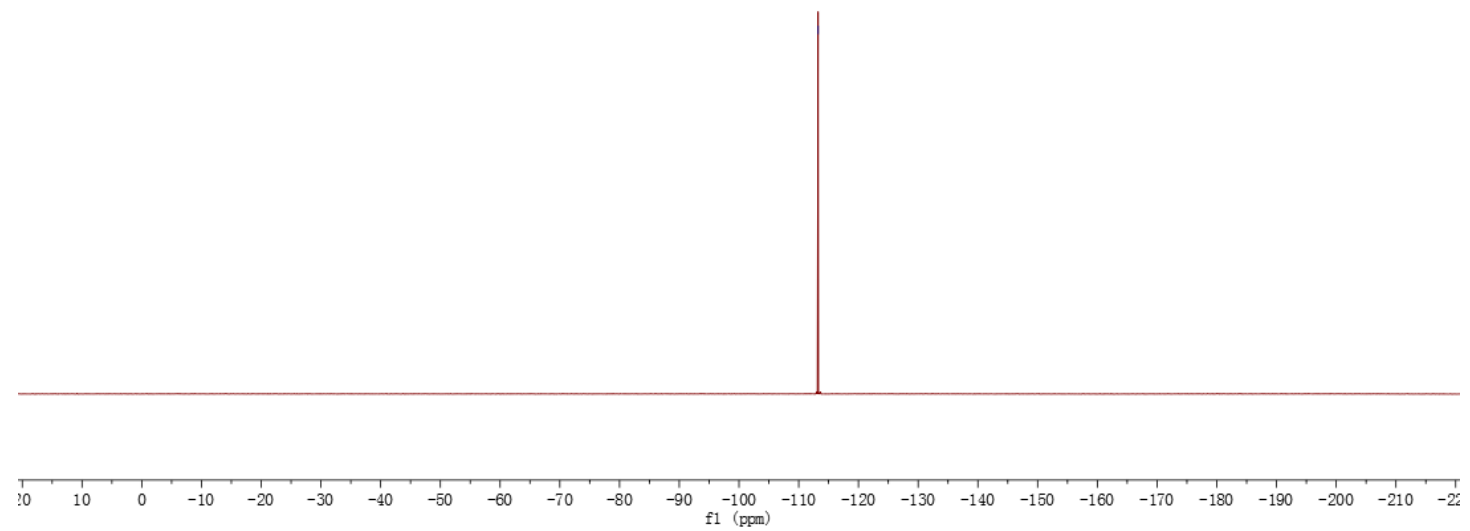

${ }^{13} \mathrm{C}$ NMR (100 MHz, $\left.\mathrm{CDCl}_{3}\right)$ of $4 \mathrm{l}$

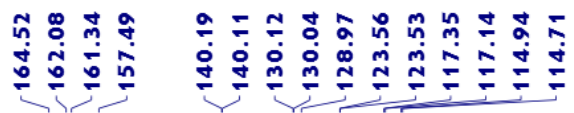
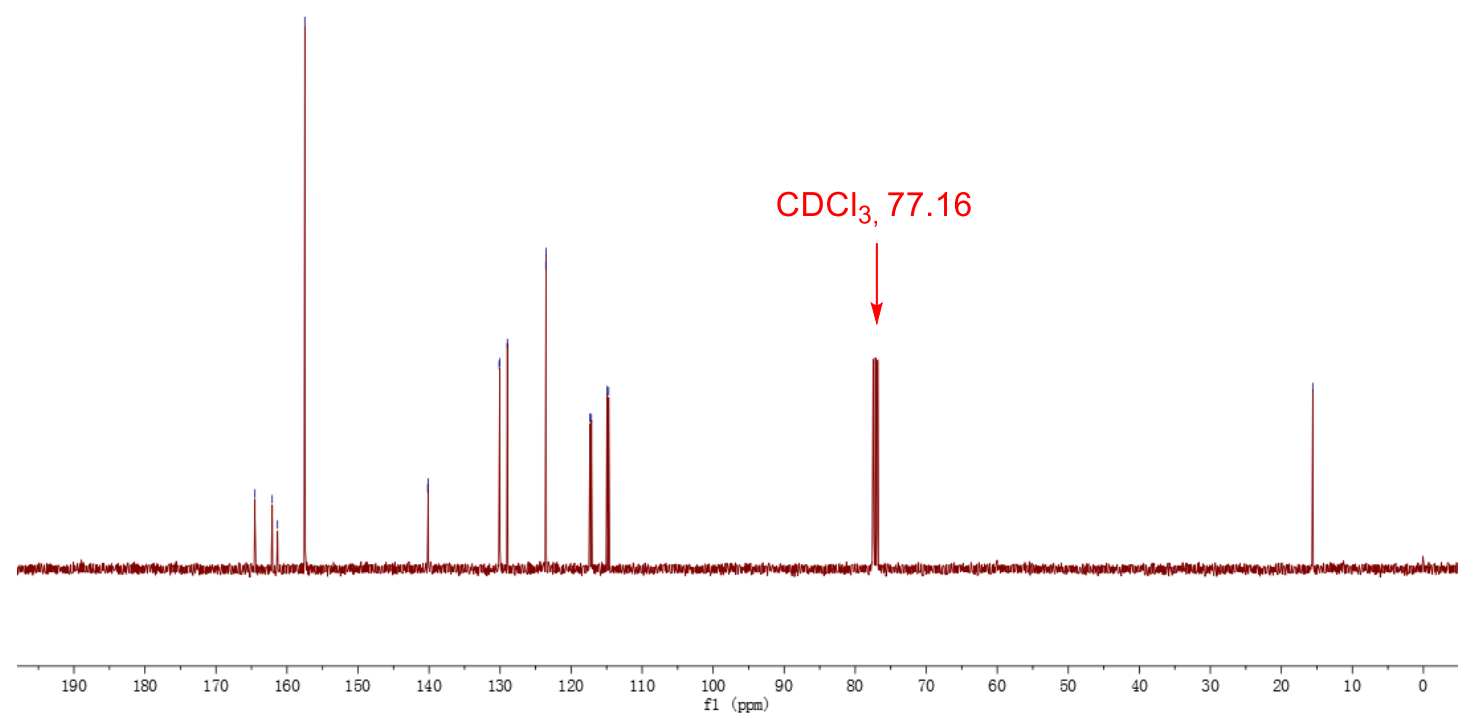
${ }^{1} \mathrm{H}$ NMR (400 MHz, $\left.\mathrm{CDCl}_{3}\right)$ of $4 \mathrm{~m}$

空

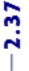

$\mathrm{CDCl}_{3}, 7.26$

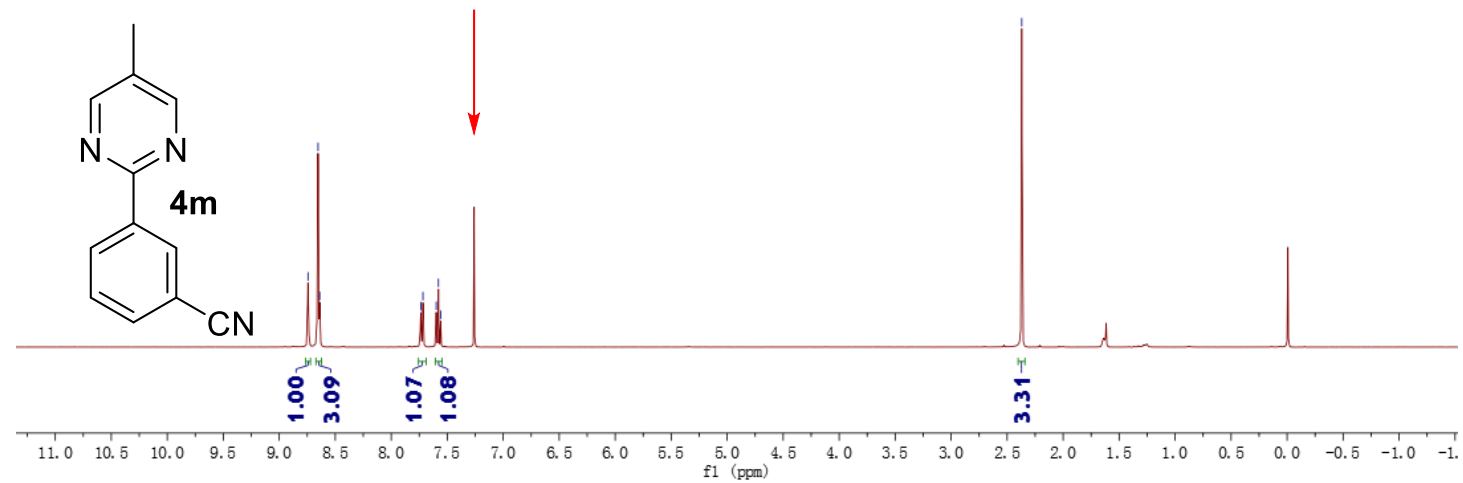

${ }^{13} \mathrm{C}$ NMR (100 MHz, CDCl 3$)$ of $4 \mathrm{~m}$

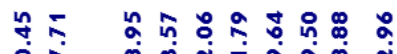

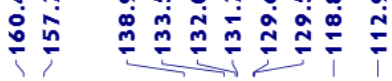

$\mathrm{CDCl}_{3}, 77.16$

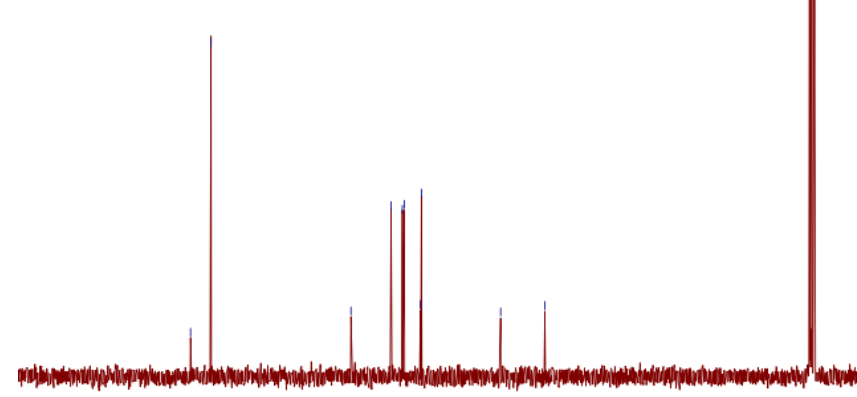

$\underset{180}{170} \quad$\begin{tabular}{llllllll}
160 & 150 & 140 & 130 & 120 & 110 & 100 & $\substack{90 \\
f 1(\mathrm{ppm})}$ \\
\hline
\end{tabular} 
${ }^{1} \mathrm{H}$ NMR (400 MHz, $\left.\mathrm{CDCl}_{3}\right)$ of 10

商

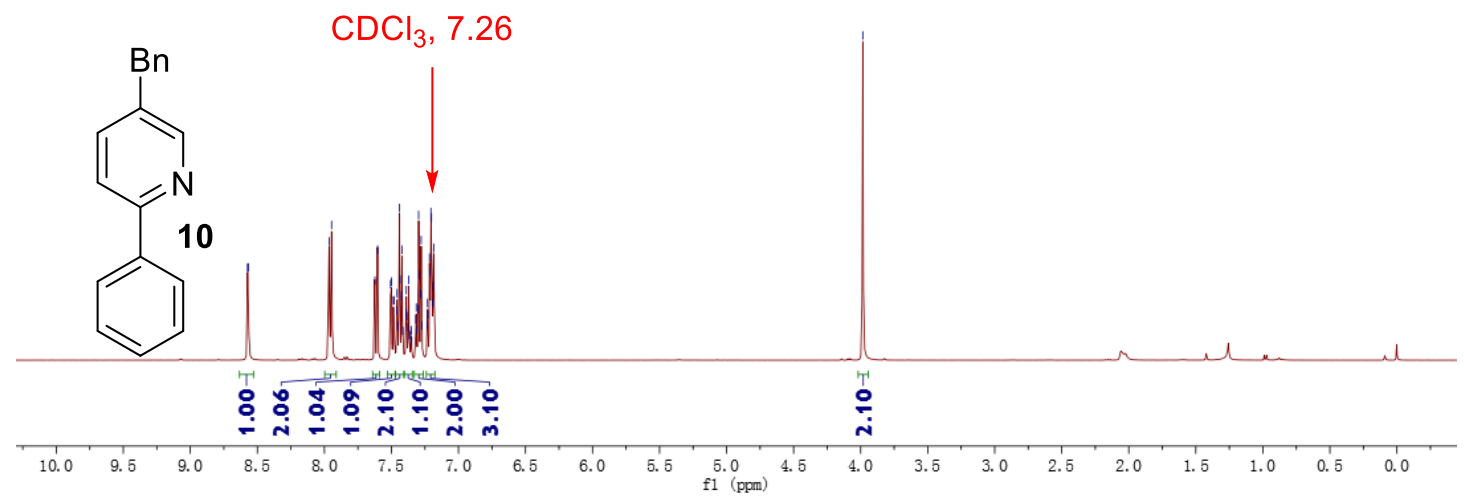

${ }^{13} \mathrm{C}$ NMR (100 MHz, $\left.\mathrm{CDCl}_{3}\right)$ of 10
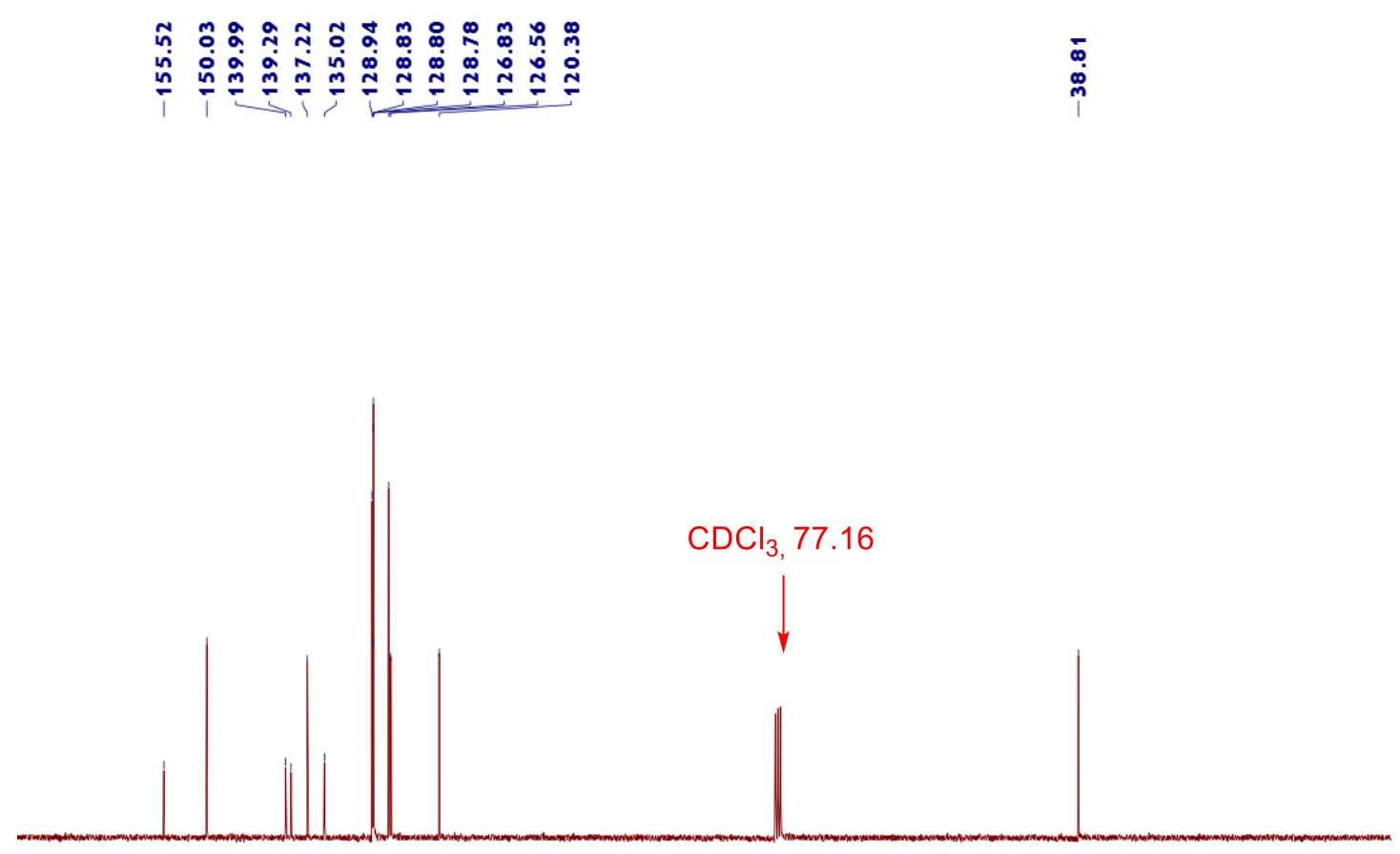

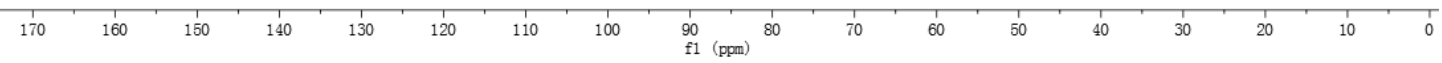


${ }^{1} \mathrm{H}$ NMR (400 MHz, $\left.\mathrm{CDCl}_{3}\right)$ of $4 \mathrm{~s}$

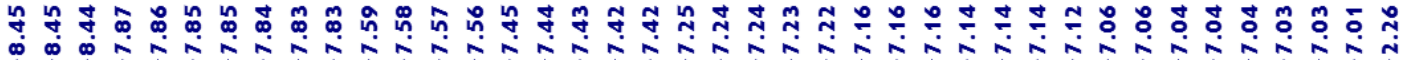

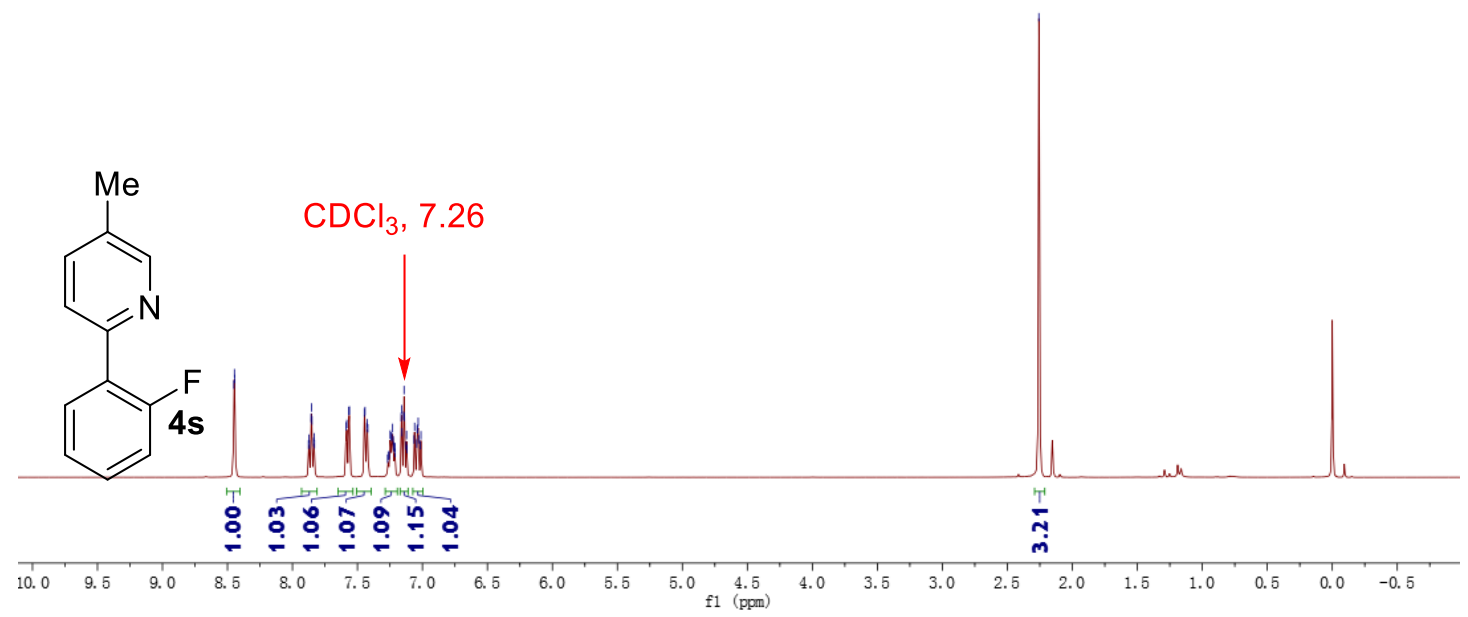

${ }^{19} \mathrm{~F}$ NMR (376 MHz, $\left.\mathrm{CDCl}_{3}\right)$ of $4 \mathrm{~s}$

$$
\frac{\mathfrak{g}}{i}
$$

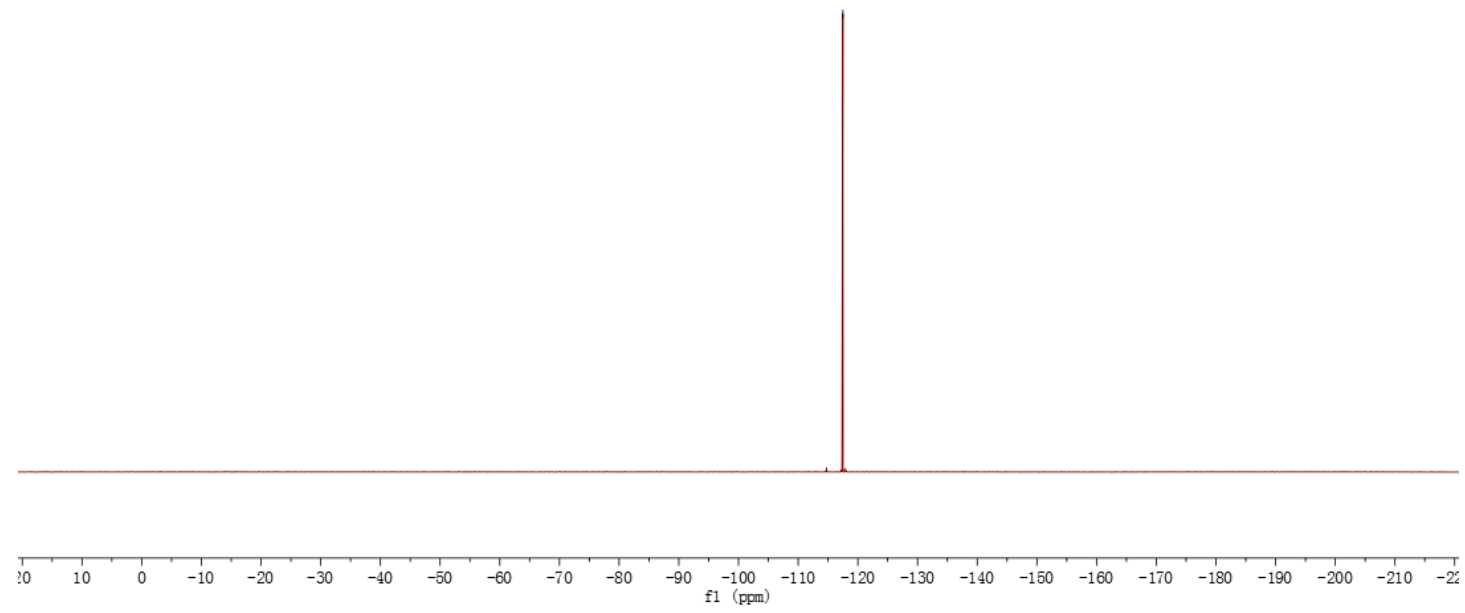


${ }^{13} \mathrm{C}$ NMR (100 $\left.\mathrm{MHz}, \mathrm{CDCl}_{3}\right)$ of $4 \mathrm{~s}$

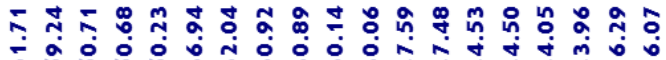

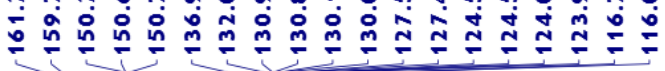

$\stackrel{\text { n }}{\stackrel{\infty}{\infty}} \underset{1}{\Gamma}$
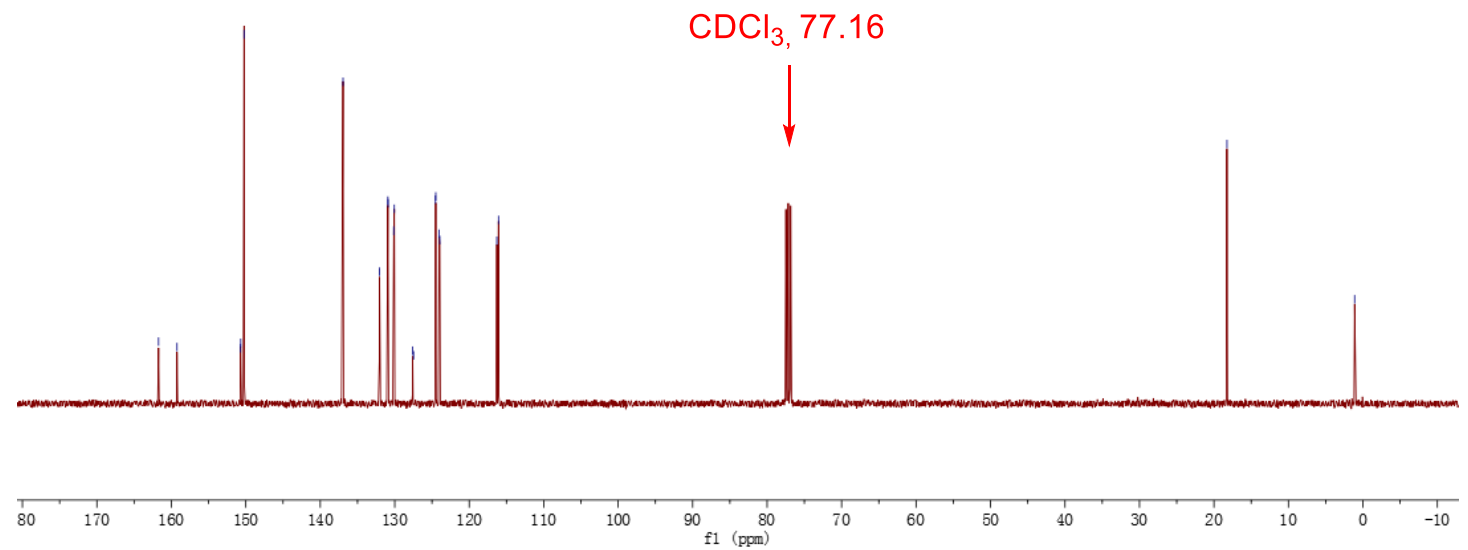

${ }^{1} \mathrm{H}$ NMR (400 MHz, CDCl 3$)$ of $4 \mathrm{t}$

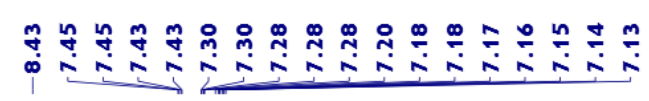

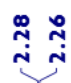

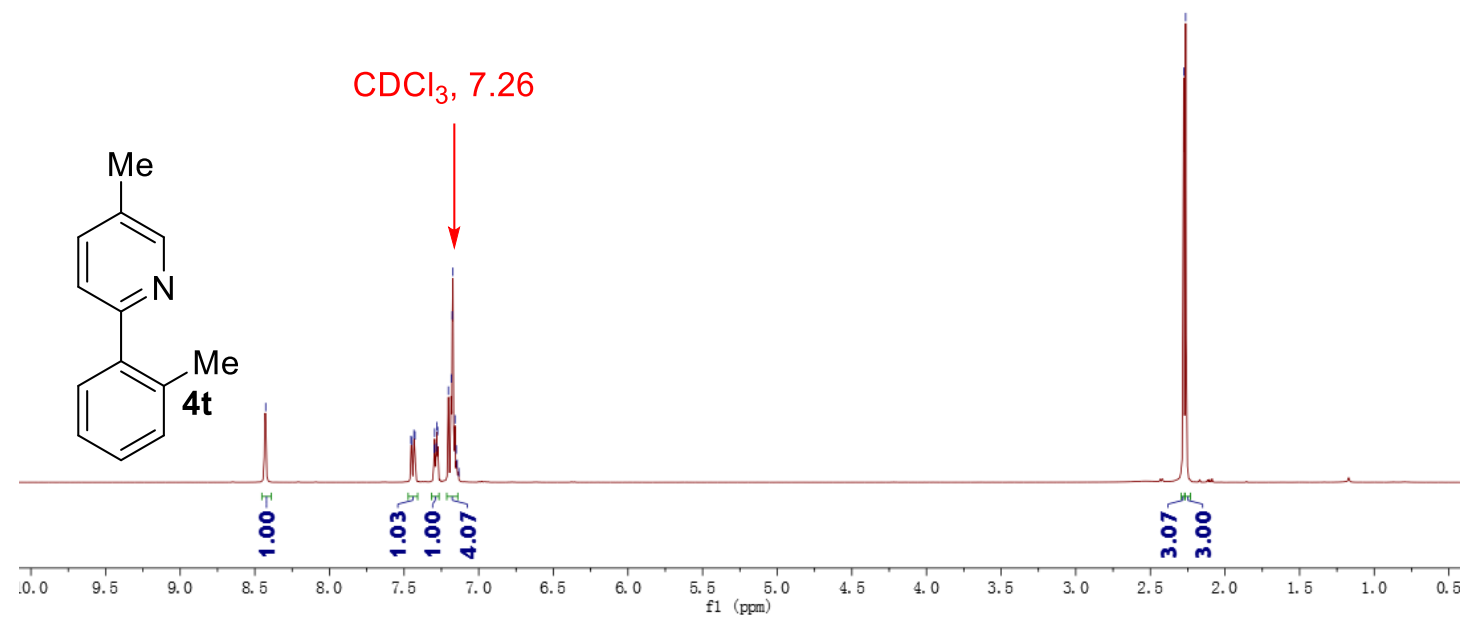


${ }^{13} \mathrm{C}$ NMR (100 MHz, $\left.\mathrm{CDCl}_{3}\right)$ of $4 \mathrm{t}$

ำ ㅇำ

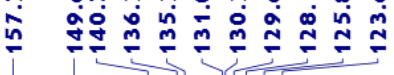

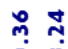

$\stackrel{1}{\dot{1}}$

$\mathrm{CDCl}_{3}, 77.16$

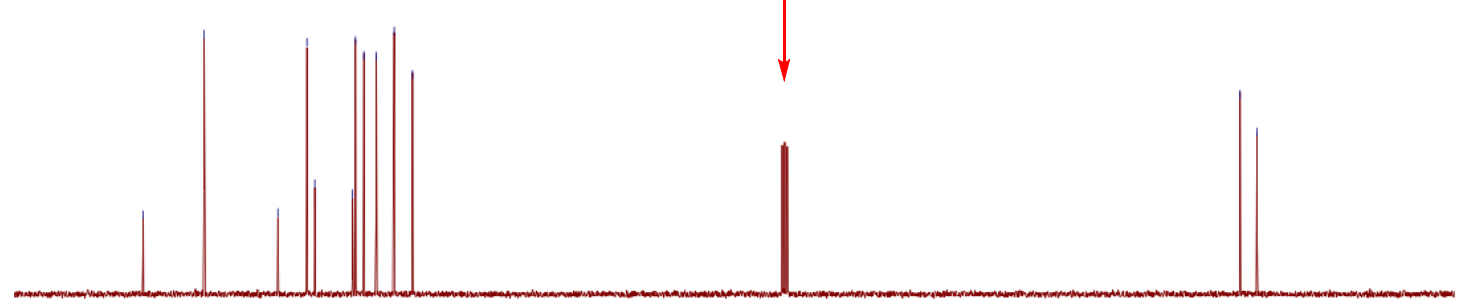

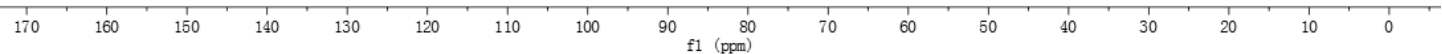

${ }^{1} \mathrm{H}$ NMR (400 MHz, $\left.\mathrm{CDCl}_{3}\right)$ of 4a-[D5]

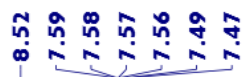

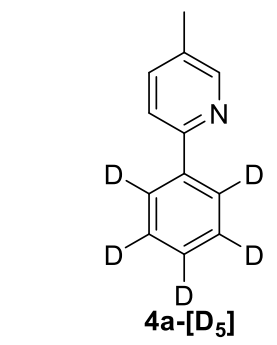

$\mathrm{CDCl}_{3}, 7.26$

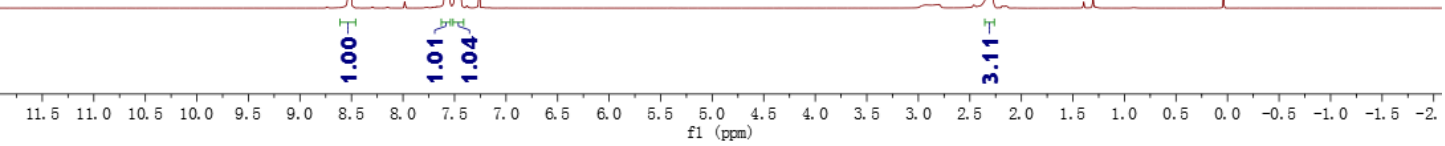


${ }^{13} \mathrm{C}$ NMR (100 MHz, $\left.\mathrm{CDCl}_{3}\right)$ of $4 \mathrm{a}-\left[\mathrm{D}_{5}\right]$

$\overline{0}$

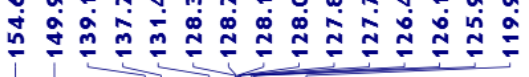

$\stackrel{\substack{\infty \\ \dot{\infty}}}{i}$

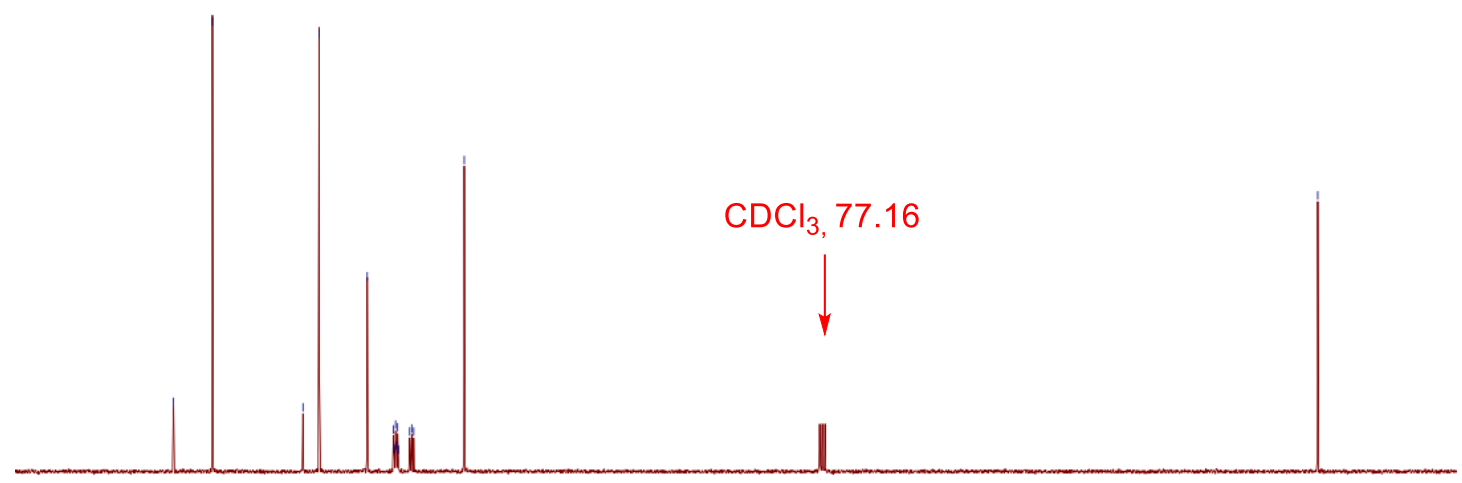

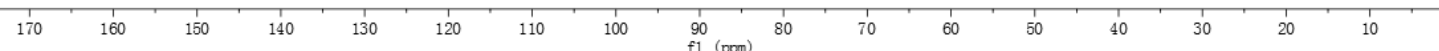


${ }^{1} \mathrm{H}$ NMR (400 MHz, $\left.\mathrm{CDCl}_{3}\right)$ of $3 \mathrm{a}$

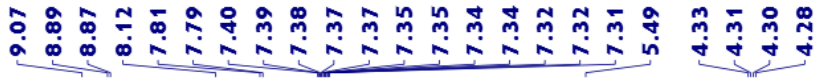
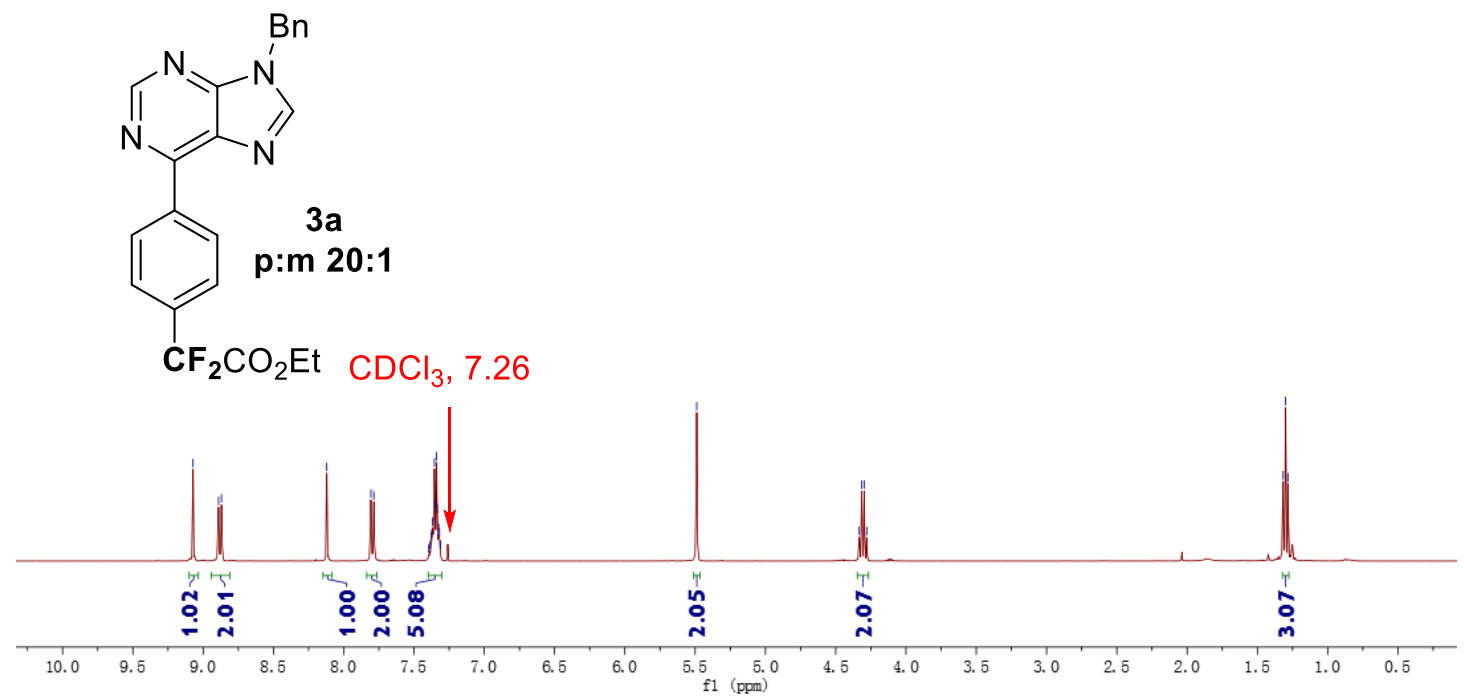

${ }^{19} \mathrm{~F}$ NMR (376 $\left.\mathrm{MHz}, \mathrm{CDCl}_{3}\right)$ of $3 \mathrm{a}$

$$
\stackrel{m}{m} \underset{j}{j}
$$

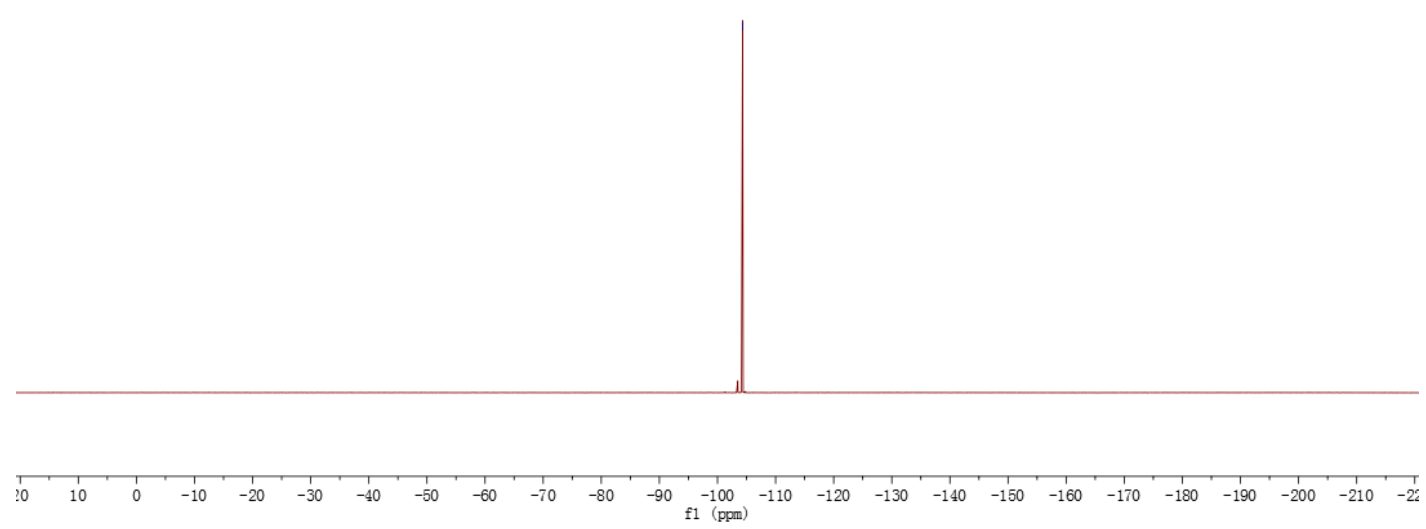


${ }^{13} \mathrm{C}$ NMR (100 MHz, $\left.\mathrm{CDCl}_{3}\right)$ of $3 \mathrm{a}$

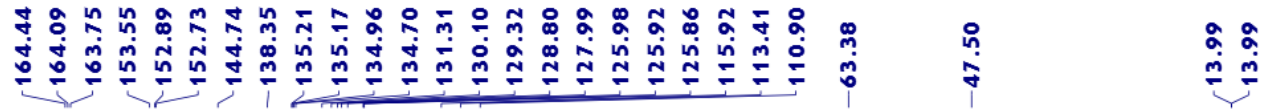

$\mathrm{CDCl}_{3}, 77.16$

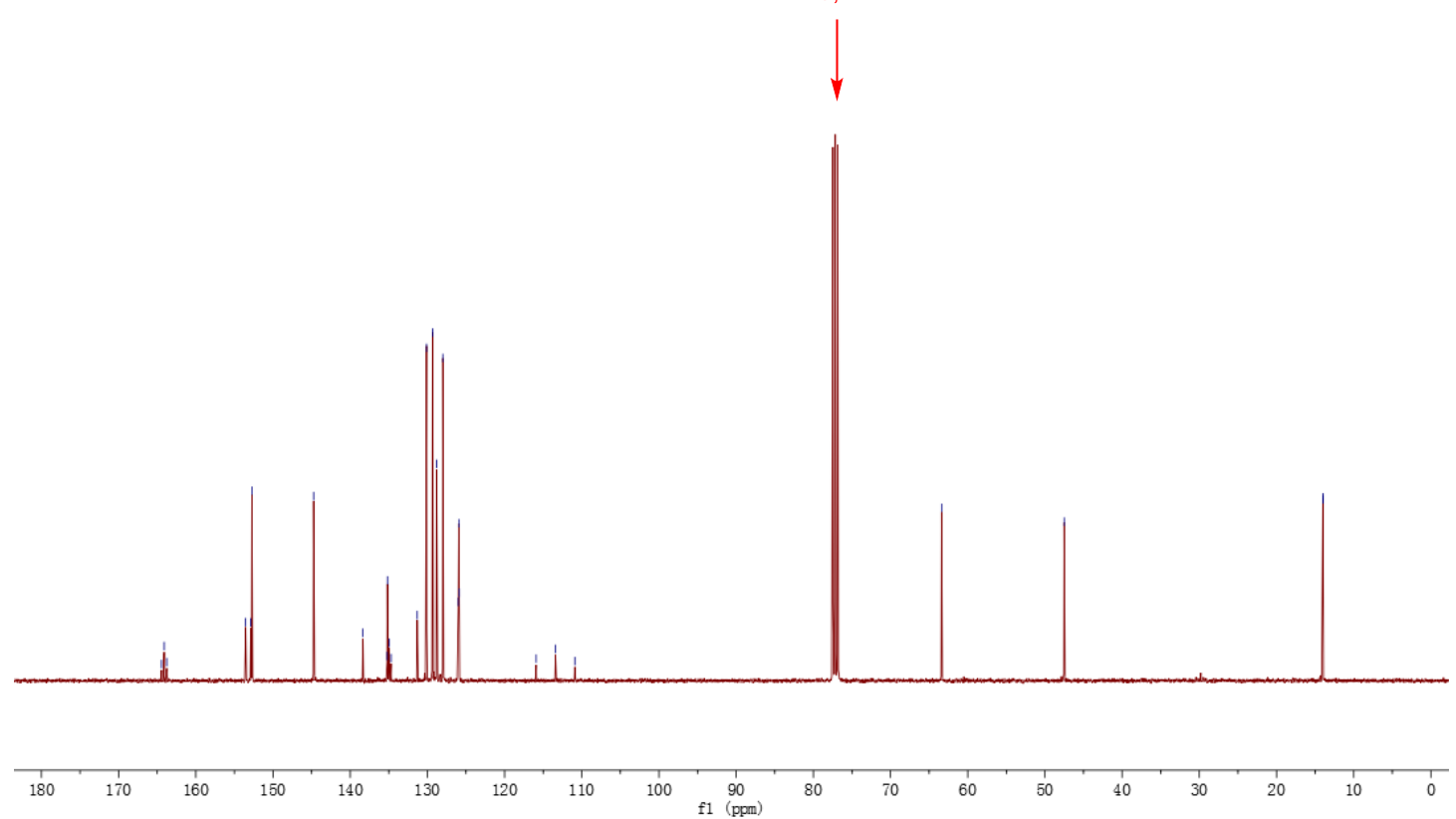

${ }^{1} \mathrm{H}$ NMR (400 MHz, $\left.\mathrm{CDCl}_{3}\right)$ of $3 \mathrm{~b}$

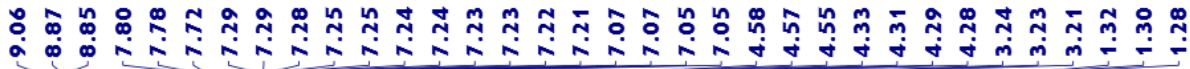

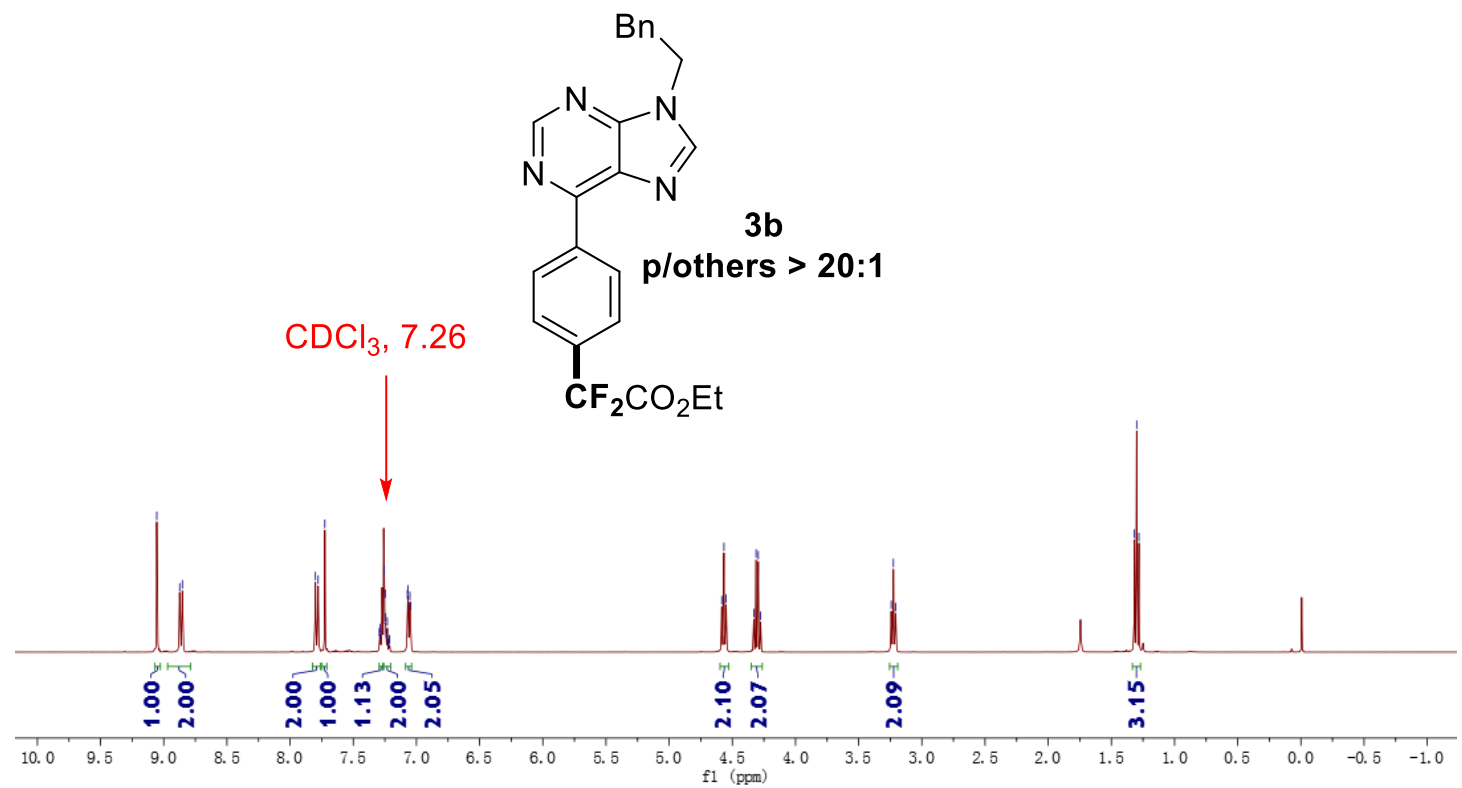


${ }^{19} \mathrm{~F}$ NMR (376 MHz, $\left.\mathrm{CDCl}_{33}\right)$ of $3 \mathrm{~b}$

$$
\stackrel{\substack{m \\ \dot{j}}}{i}
$$

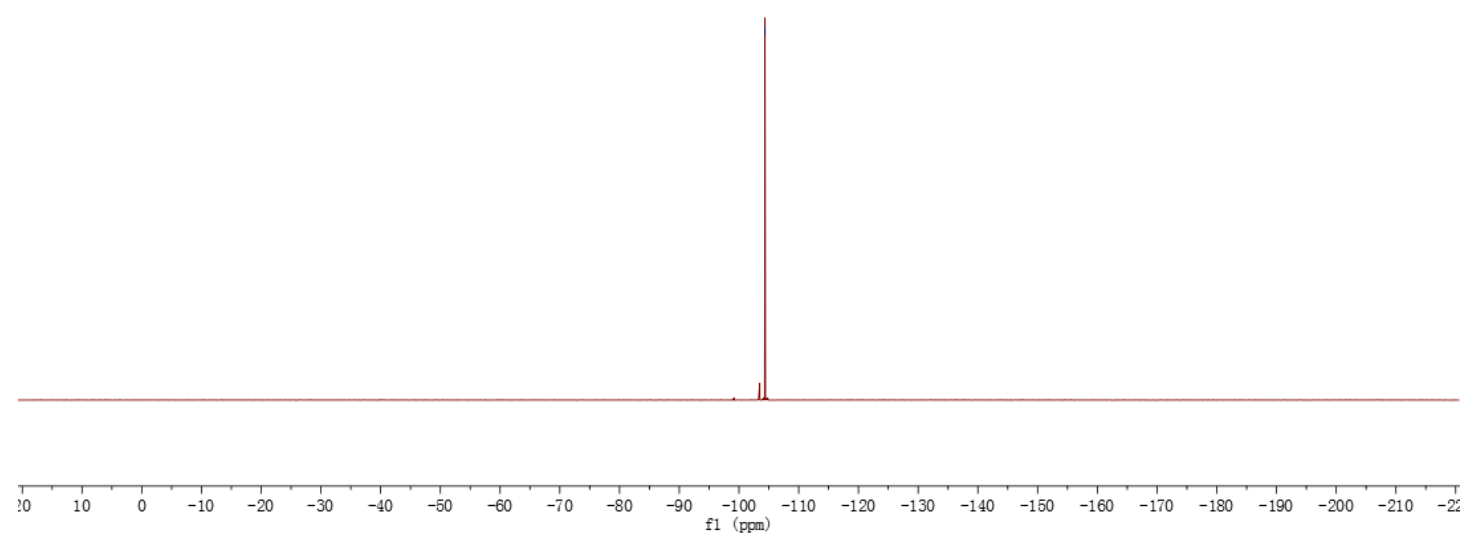

${ }^{13} \mathrm{C}$ NMR (100 MHz, $\left.\mathrm{CDCl}_{3}\right)$ of $3 \mathrm{~b}$

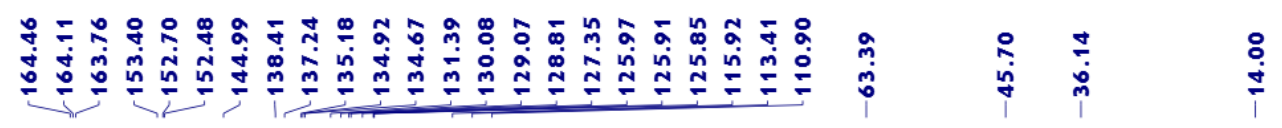
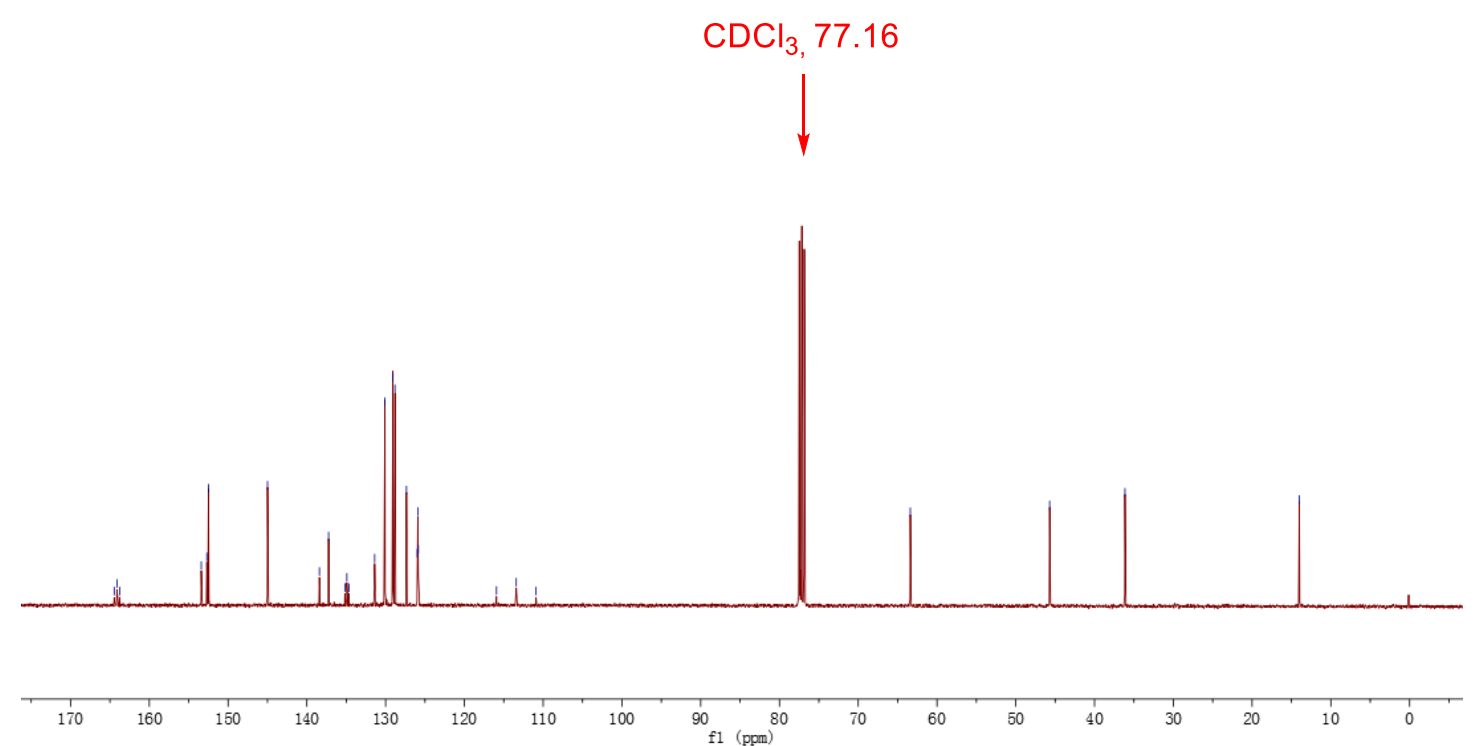
${ }^{1} \mathrm{H}$ NMR (400 MHz, $\left.\mathrm{CDCl}_{3}\right)$ of 3c

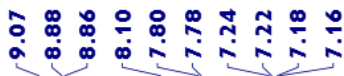
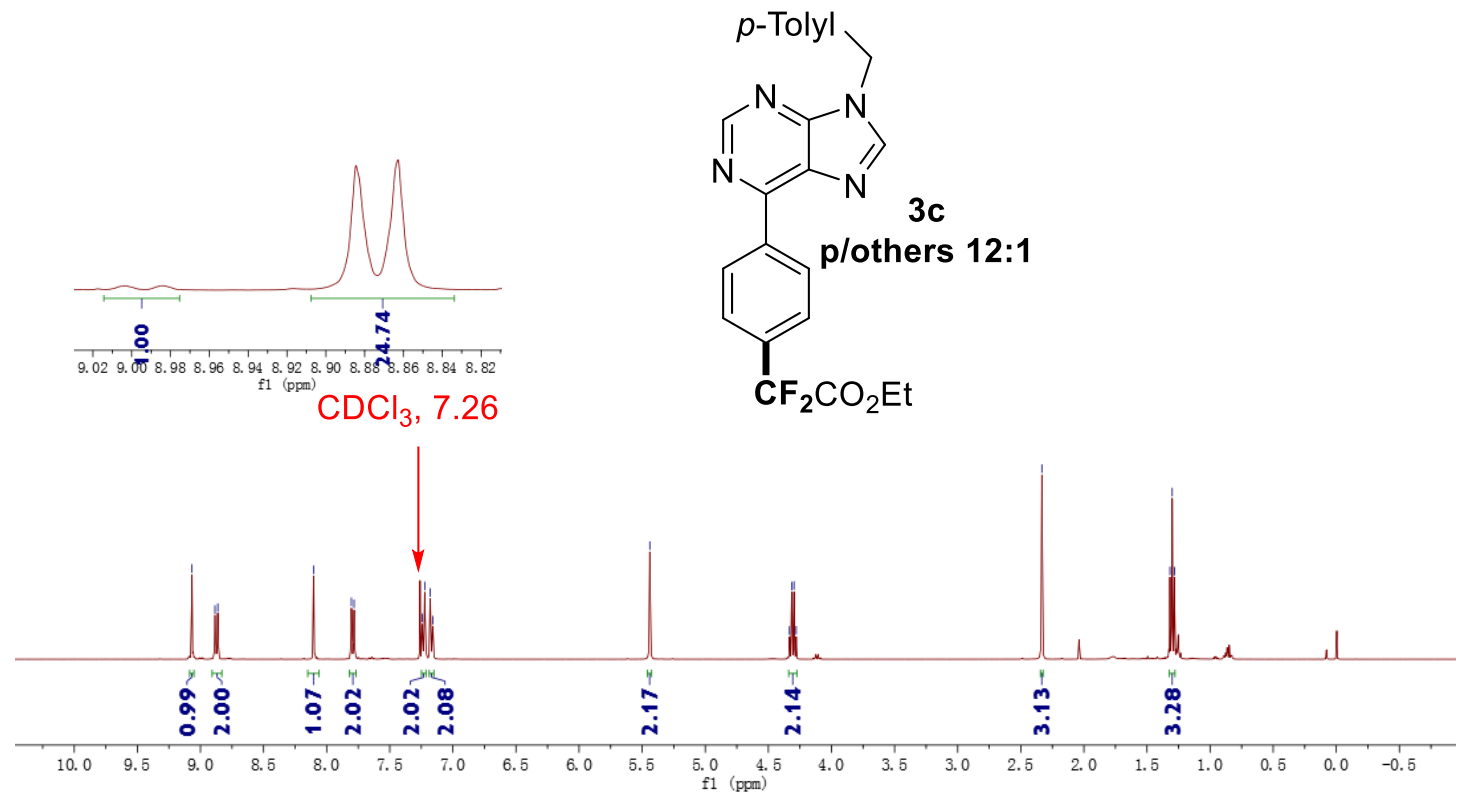

${ }^{19}$ F NMR (376 MHz, CDCl3) of 3c

$$
\stackrel{m}{m} \underset{j}{j}
$$

\begin{tabular}{lllllllllllllllllllllllllllllllll}
1 & 10 & 10 & 0 & -10 & -20 & -30 & -40 & -50 & -60 & -70 & -80 & -90 & -100 & -110 & -120 & -130 & -140 & -150 & -160 & -170 & -180 & -190 & -200 & -210 & -22 \\
\hline
\end{tabular} 
${ }^{13} \mathrm{C}$ NMR (100 MHz, $\left.\mathrm{CDCl}_{3}\right)$ of $3 \mathrm{c}$

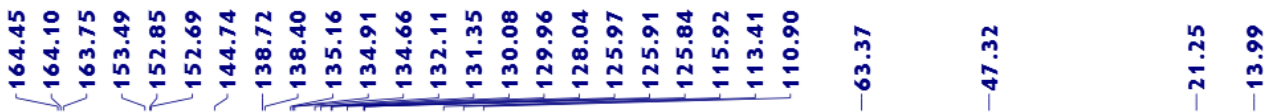

$\mathrm{CDCl}_{3,}, 77.16$
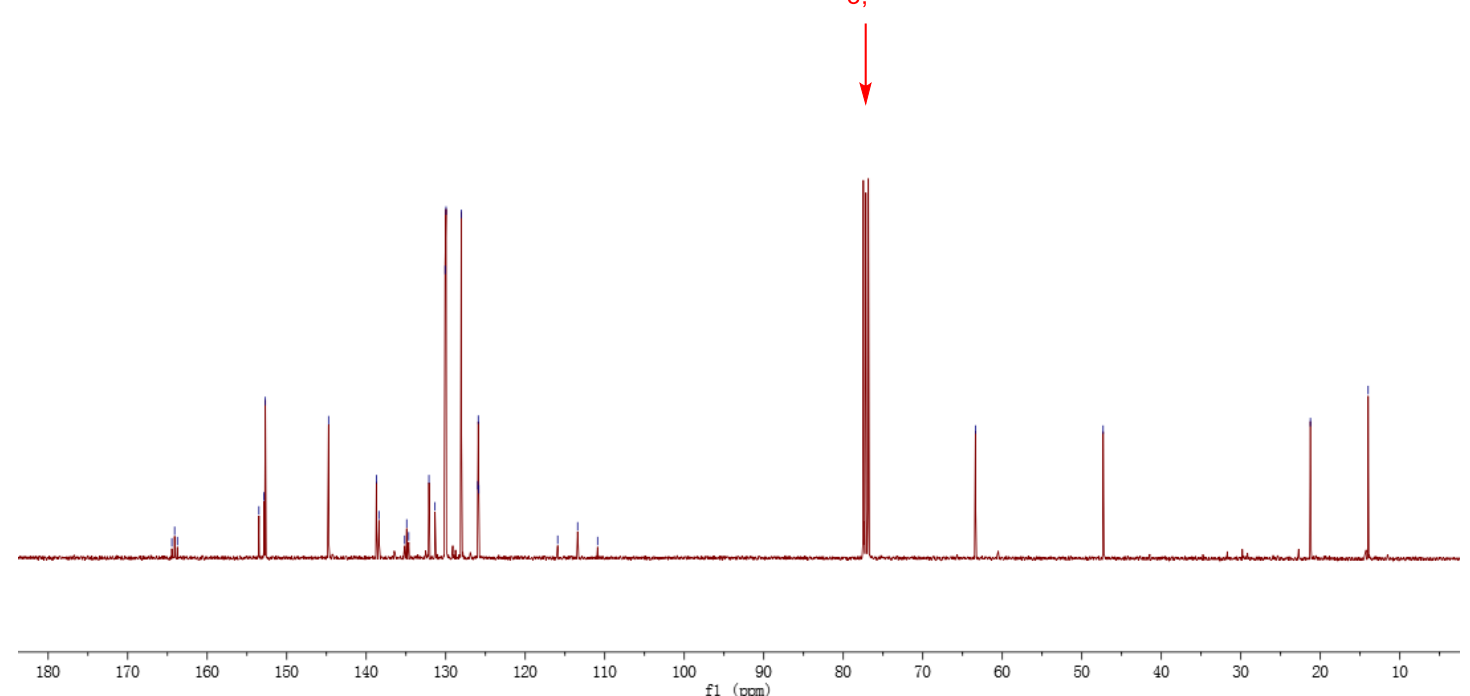

${ }^{1} \mathrm{H}$ NMR (400 MHz, $\left.\mathrm{CDCl}_{3}\right)$ of $3 \mathrm{~d}$

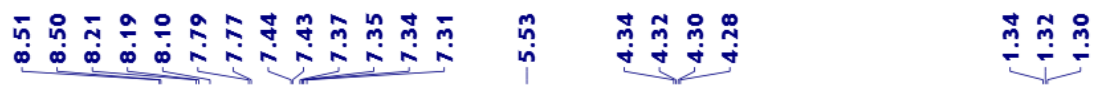
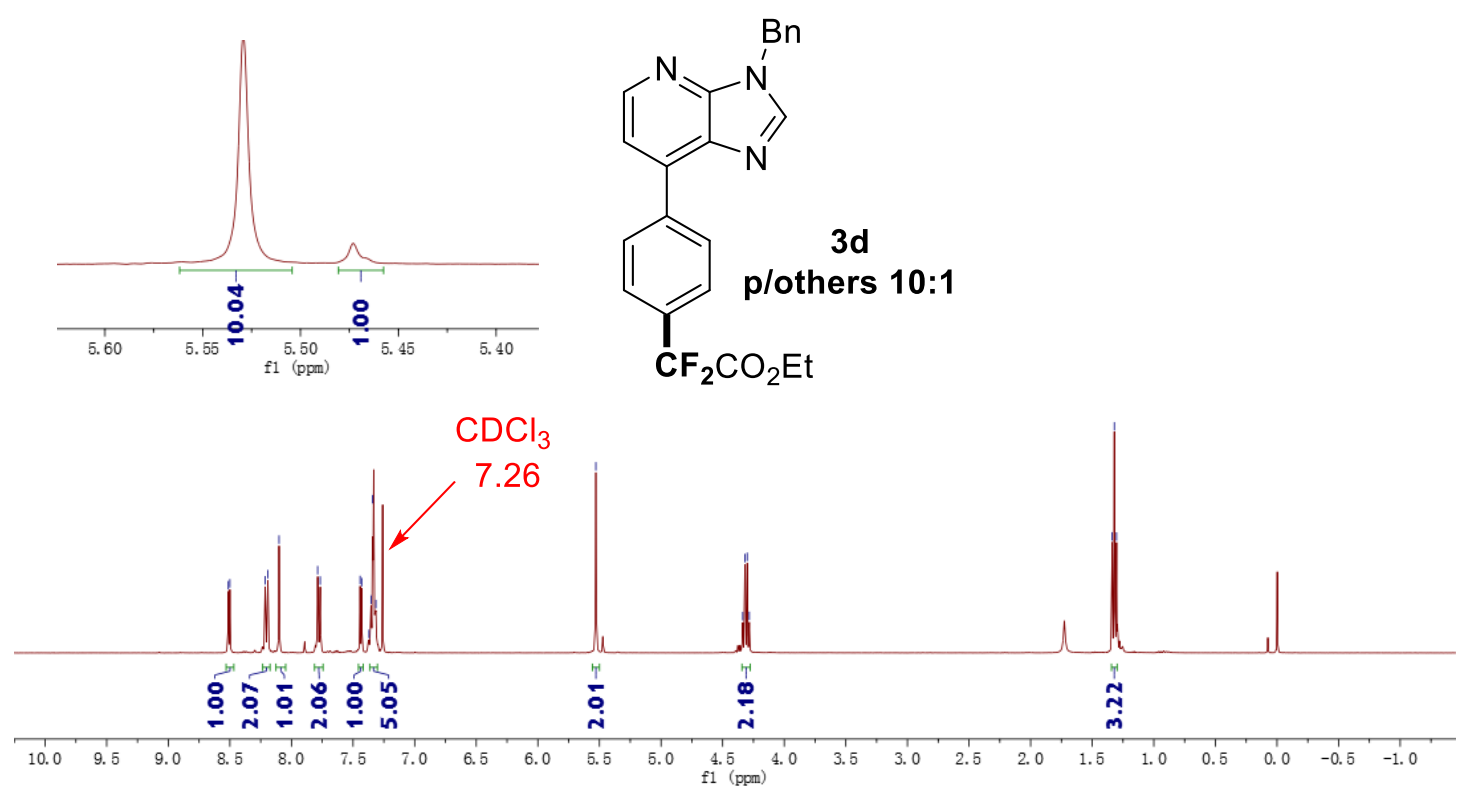
${ }^{19} \mathrm{~F}$ NMR (376 $\left.\mathrm{MHz}, \mathrm{CDCl}_{3}\right)$ of 3d

$$
\text { ㅇํํ }
$$

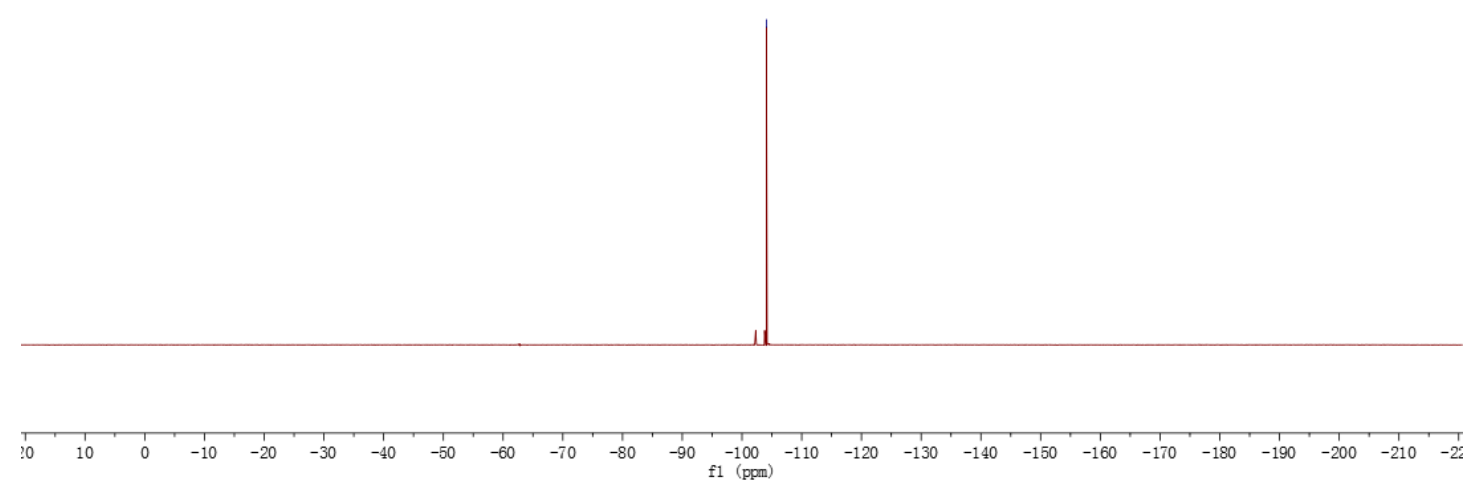

${ }^{13} \mathrm{C}$ NMR (100 MHz, $\left.\mathrm{CDCl}_{3}\right)$ of 3d

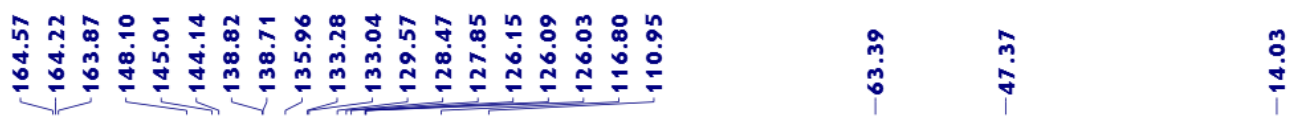

$\mathrm{CDCl}_{3}, 77.16$

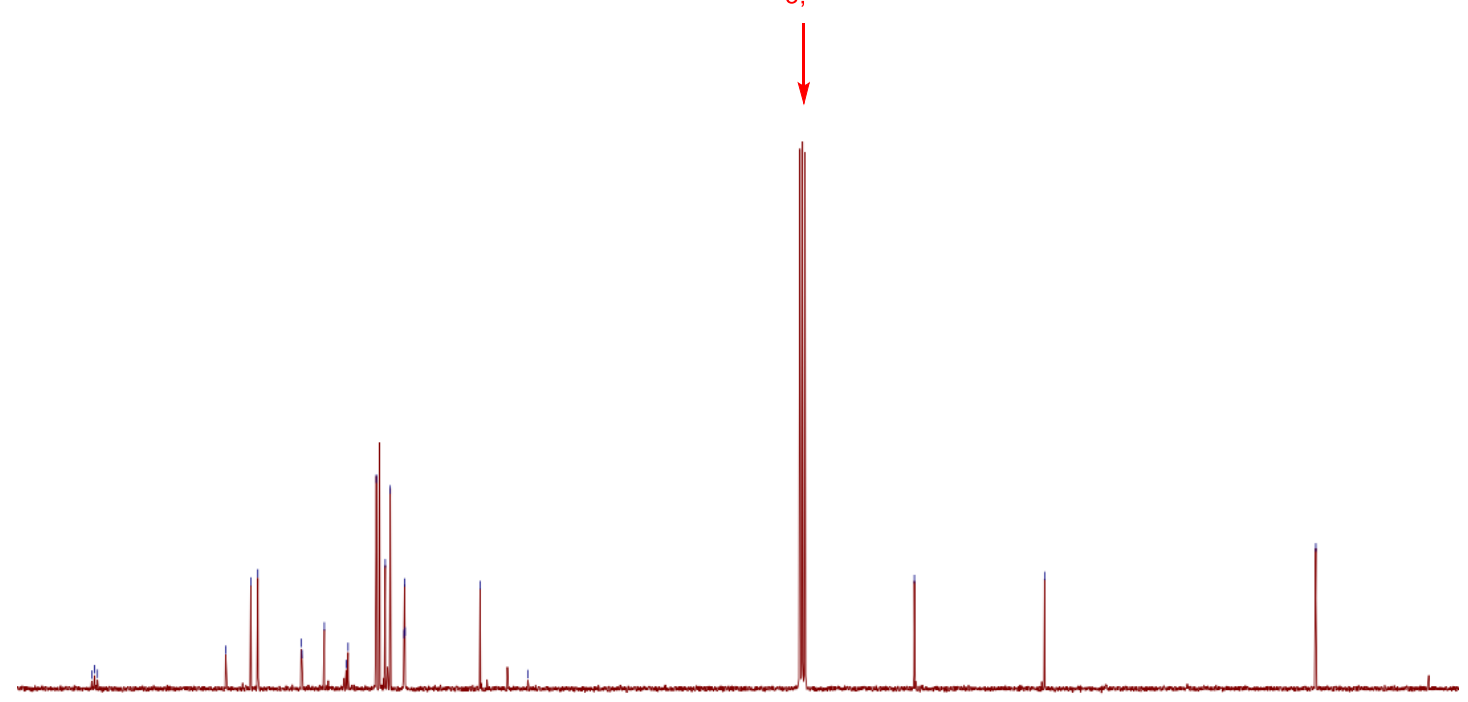

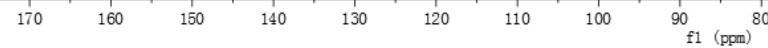


${ }^{1} \mathrm{H}$ NMR (400 MHz, CDCl 3$)$ of $3 e$

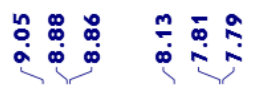
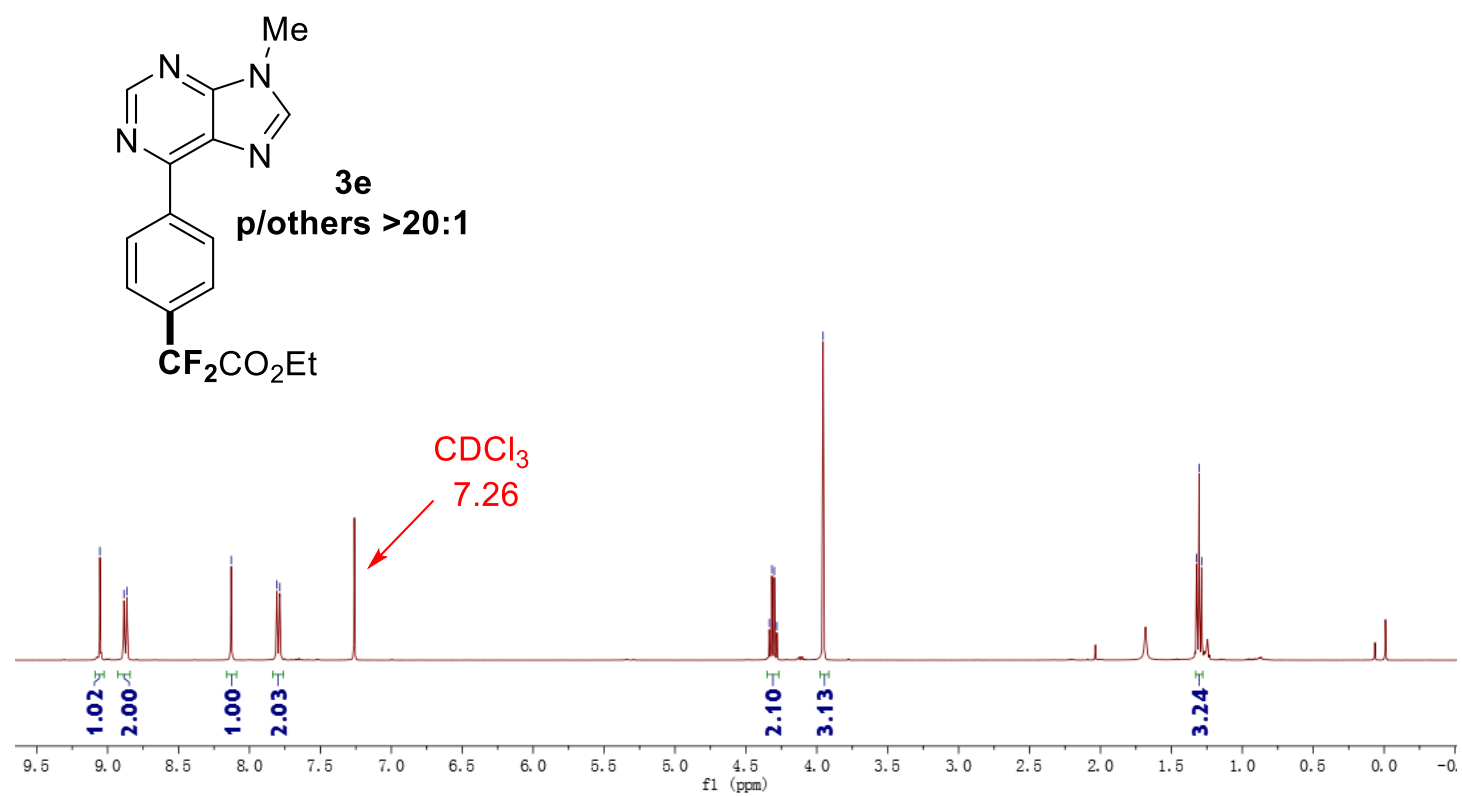

${ }^{19}$ F NMR (376 $\left.\mathrm{MHz}, \mathrm{CDCl}_{3}\right)$ of $3 \mathrm{e}$

$$
\text { ঙ̊ํ் }
$$

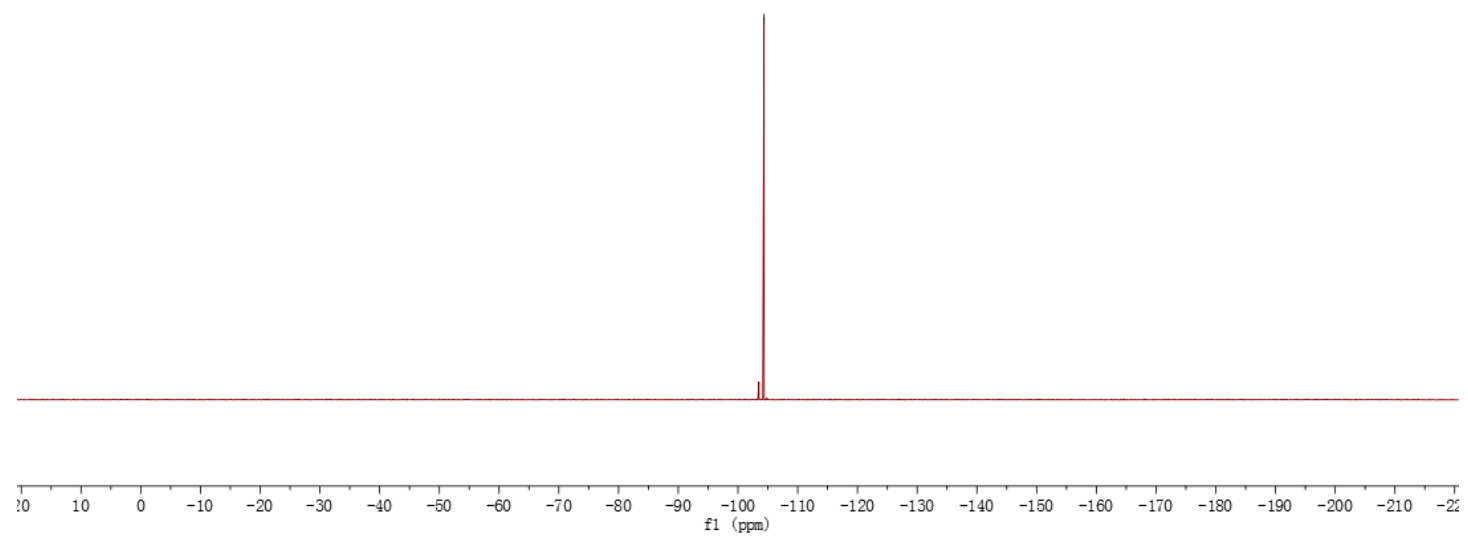


${ }^{13} \mathrm{C}$ NMR (100 MHz, $\left.\mathrm{CDCl}_{3}\right)$ of $3 \mathrm{e}$

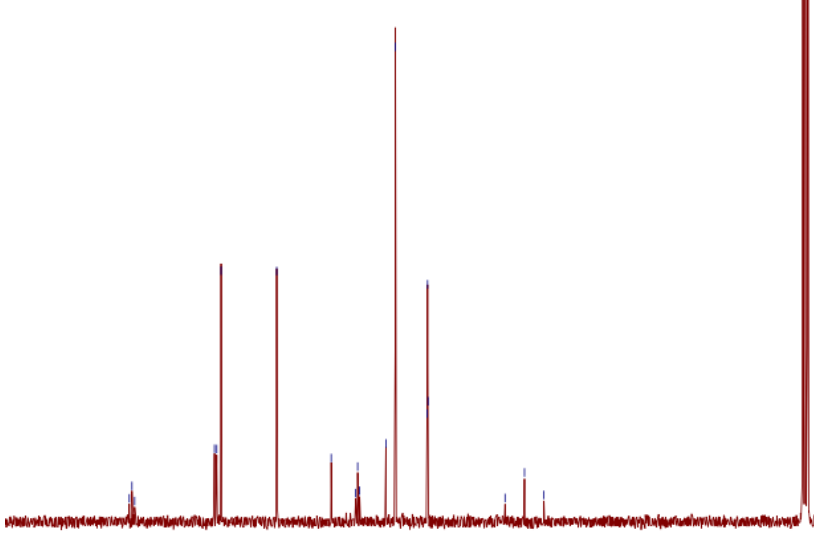

${ }^{1} \mathrm{H}$ NMR (400 MHz, CDCl $)$ of $3 f$

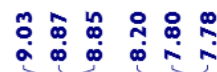
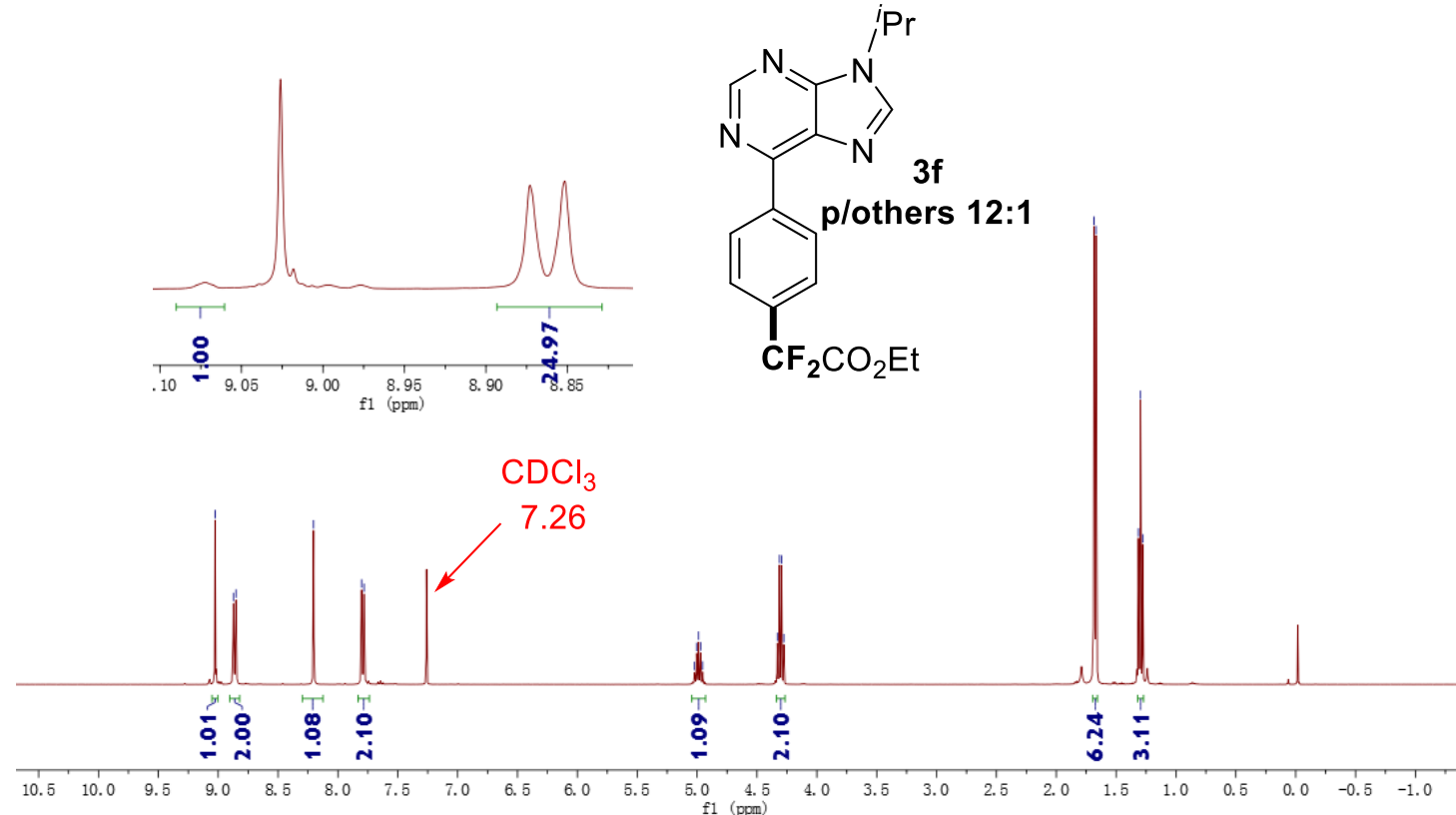
${ }^{19}$ F NMR (376 MHz, $\left.\mathrm{CDCl}_{3}\right)$ of $3 f$

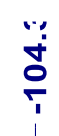

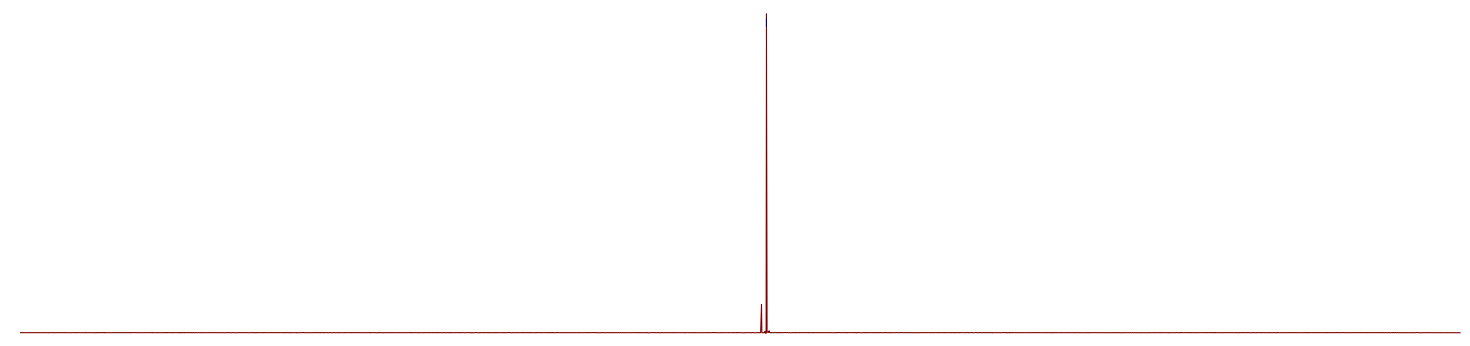

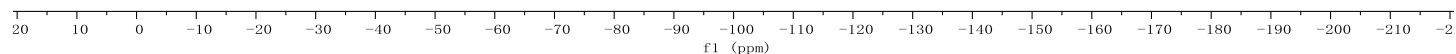

${ }^{13} \mathrm{C}$ NMR (100 MHz, $\left.\mathrm{CDCl}_{3}\right)$ of $3 f$

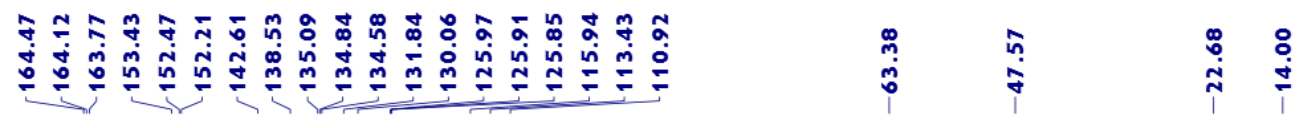

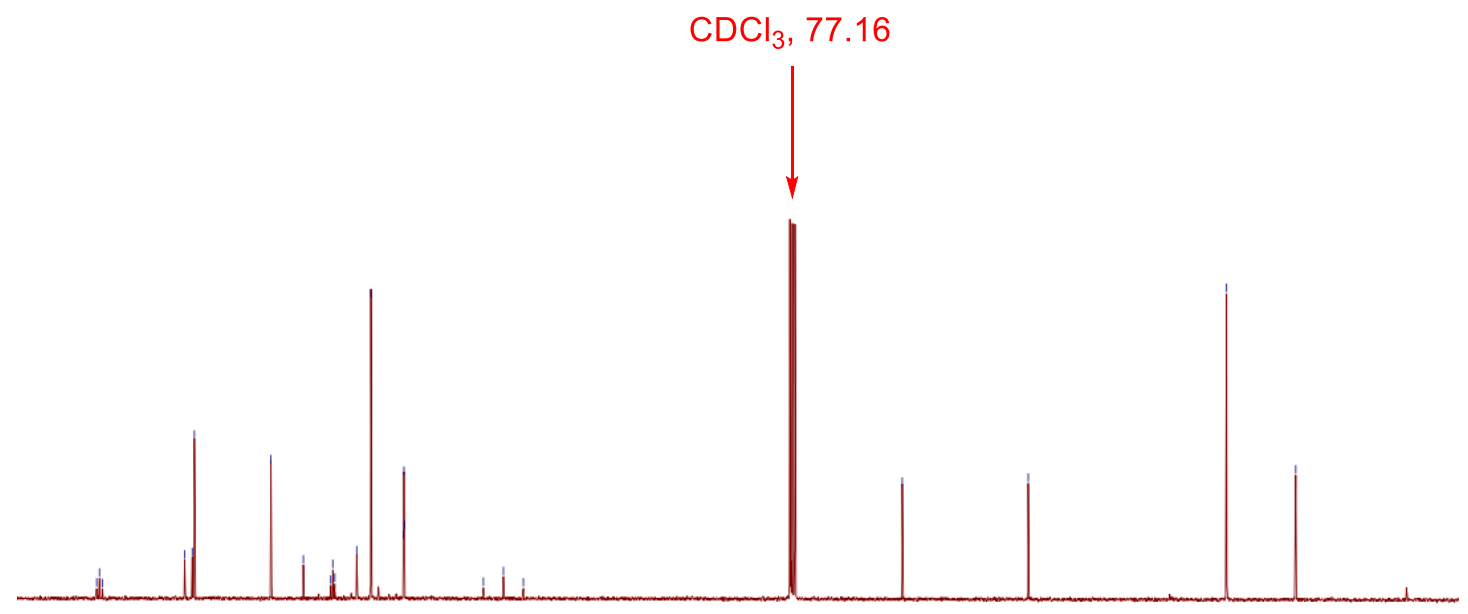

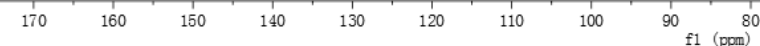


${ }^{1} \mathrm{H}$ NMR (400 MHz, CDCl 3$)$ of $3 \mathrm{~g}$

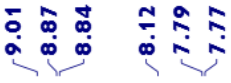

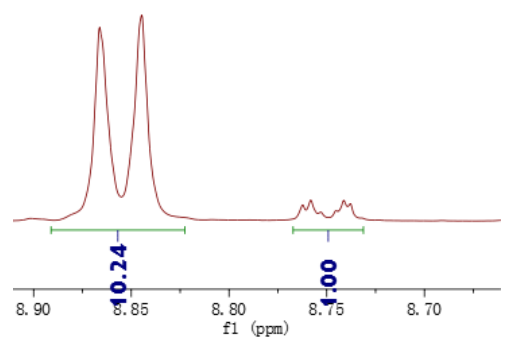

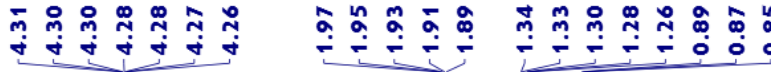

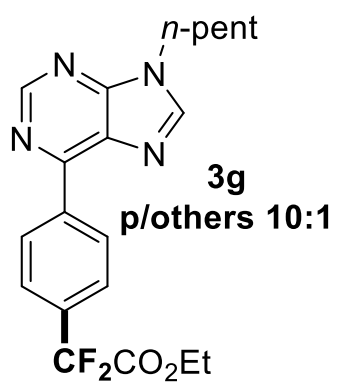

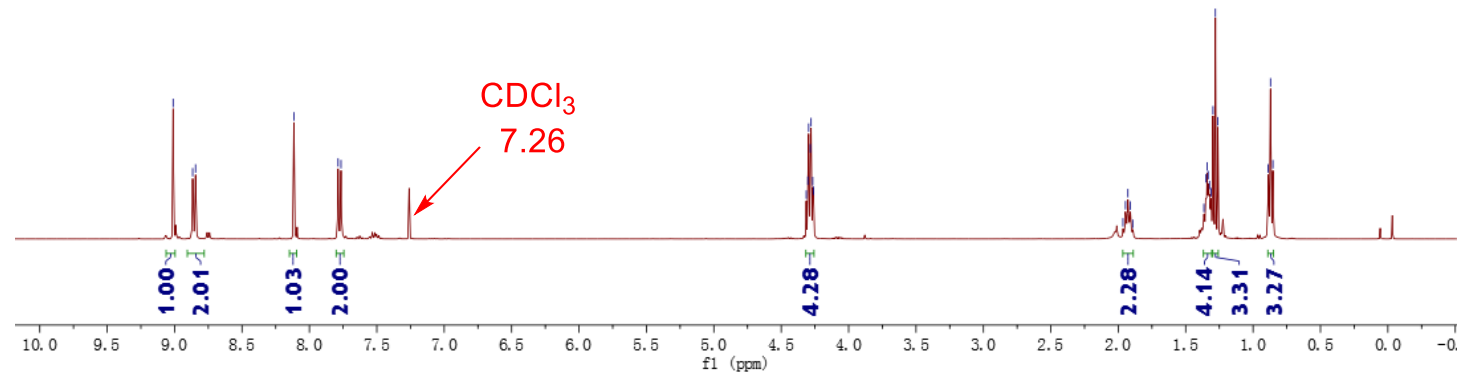

${ }^{19} \mathrm{~F}$ NMR $\left(376 \mathrm{MHz}, \mathrm{CDCl}_{3}\right)$ of $3 \mathrm{~g}$

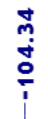

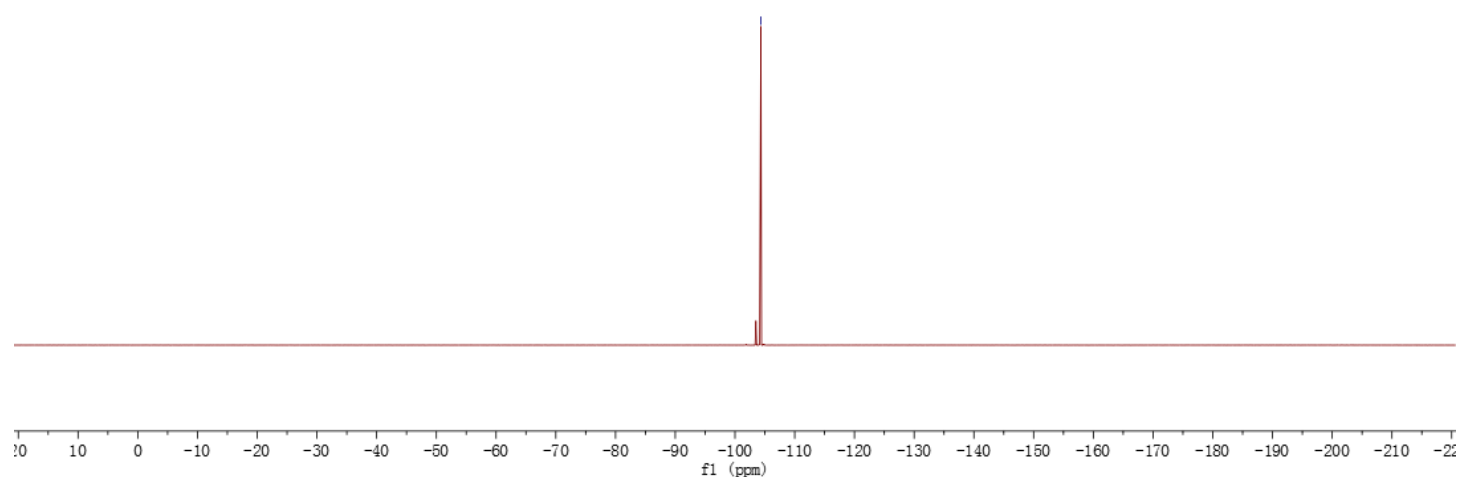


${ }^{13} \mathrm{C}$ NMR (100 MHz, $\left.\mathrm{CDCl}_{3}\right)$ of $3 \mathrm{~g}$

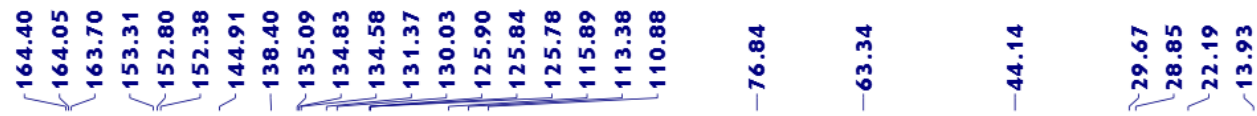

$\mathrm{CDCl}_{3}, 77.16$
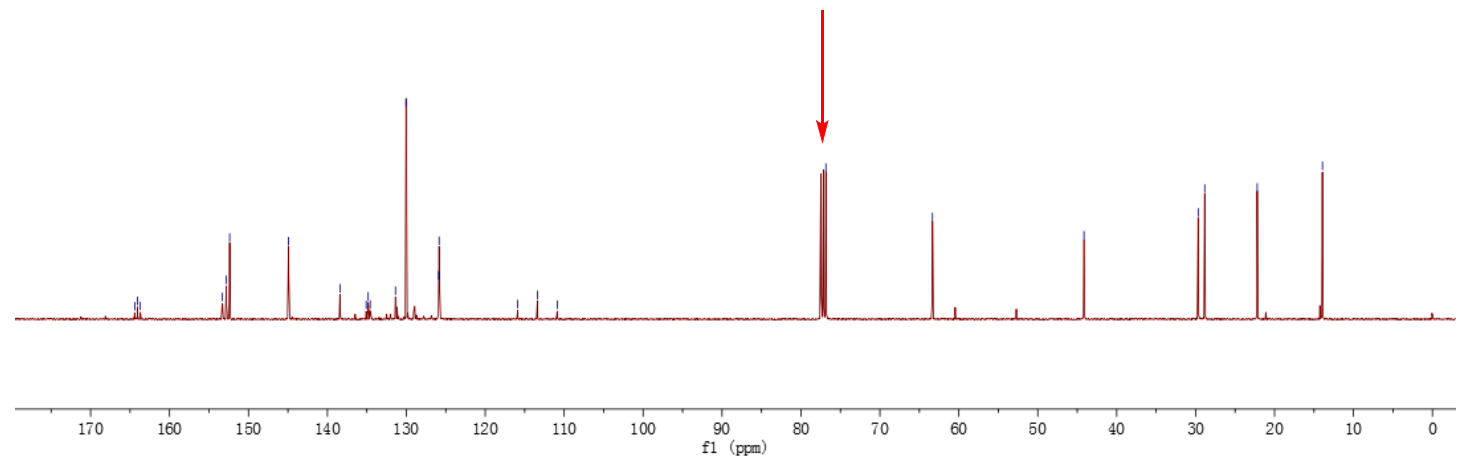

${ }^{1} \mathrm{H}$ NMR (400 MHz, CDCl $)$ of $3 \mathrm{~h}$

冓市

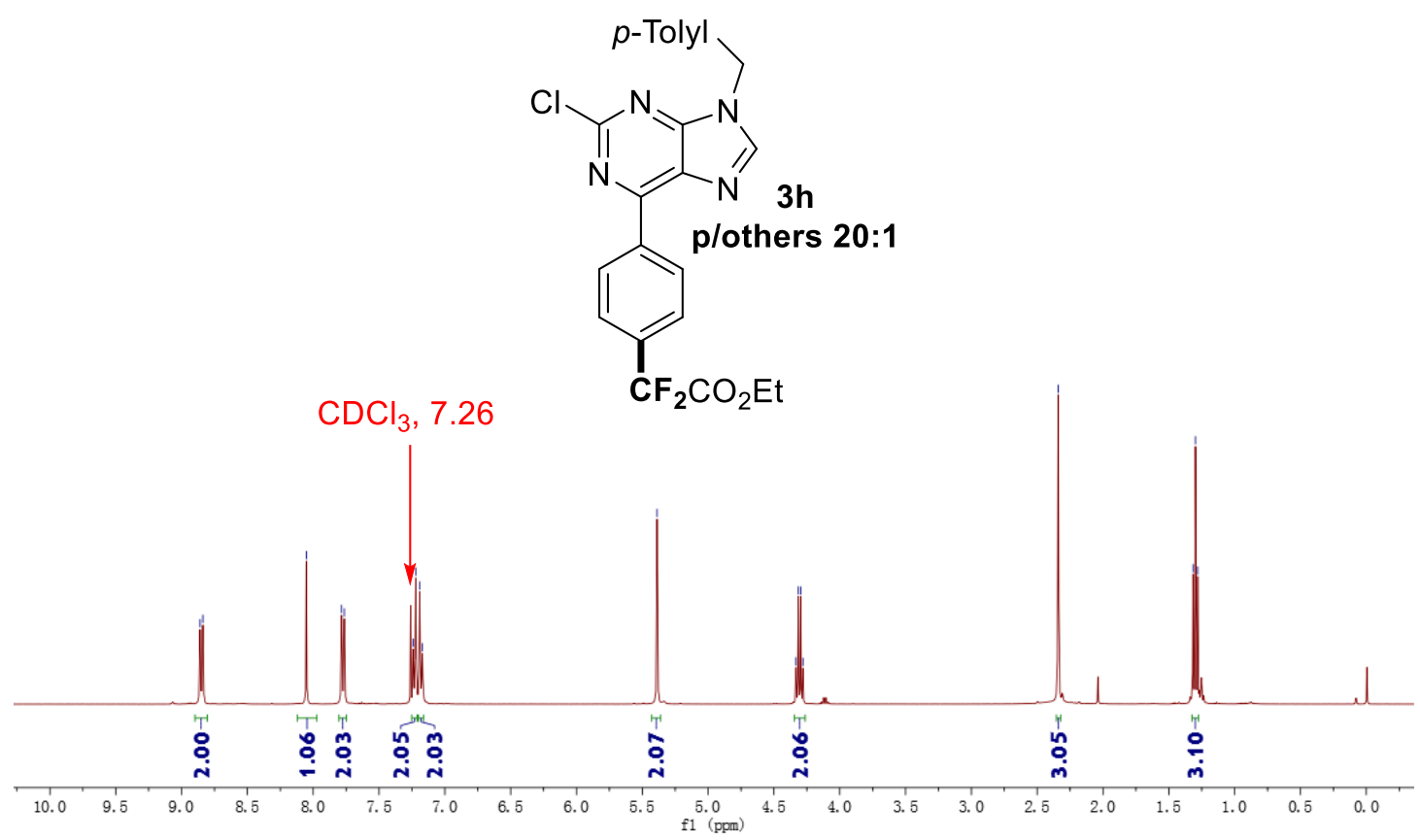


${ }^{19}$ F NMR (376 MHz, $\left.\mathrm{CDCl}_{3}\right)$ of $3 \mathrm{~h}$

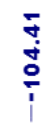

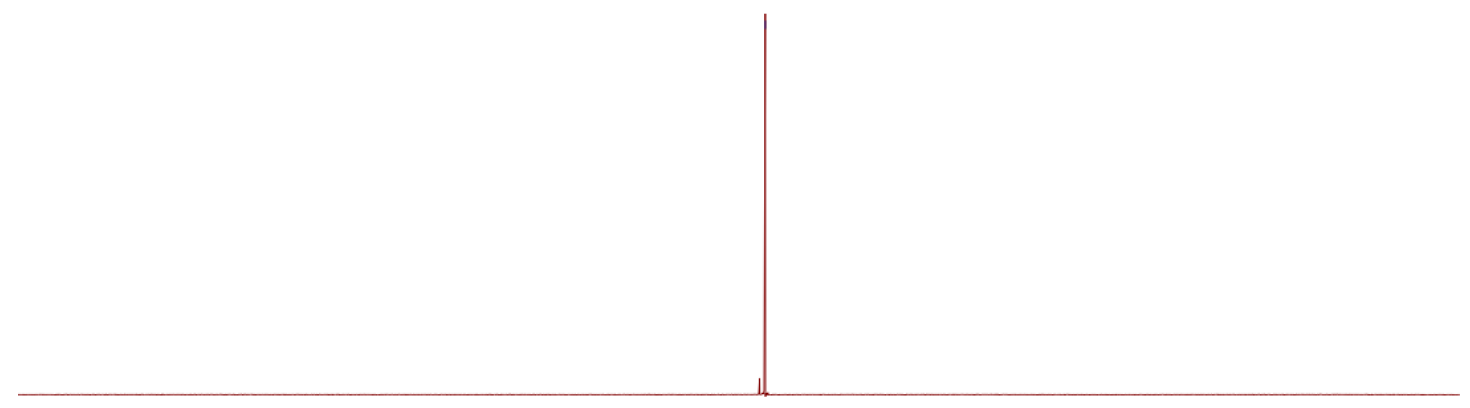

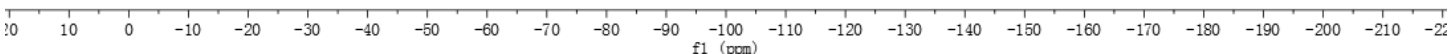

${ }^{13} \mathrm{C}$ NMR (100 MHz, $\left.\mathrm{CDCl}_{3}\right)$ of $3 \mathrm{~h}$

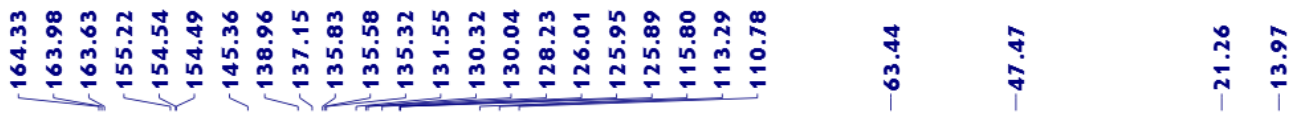

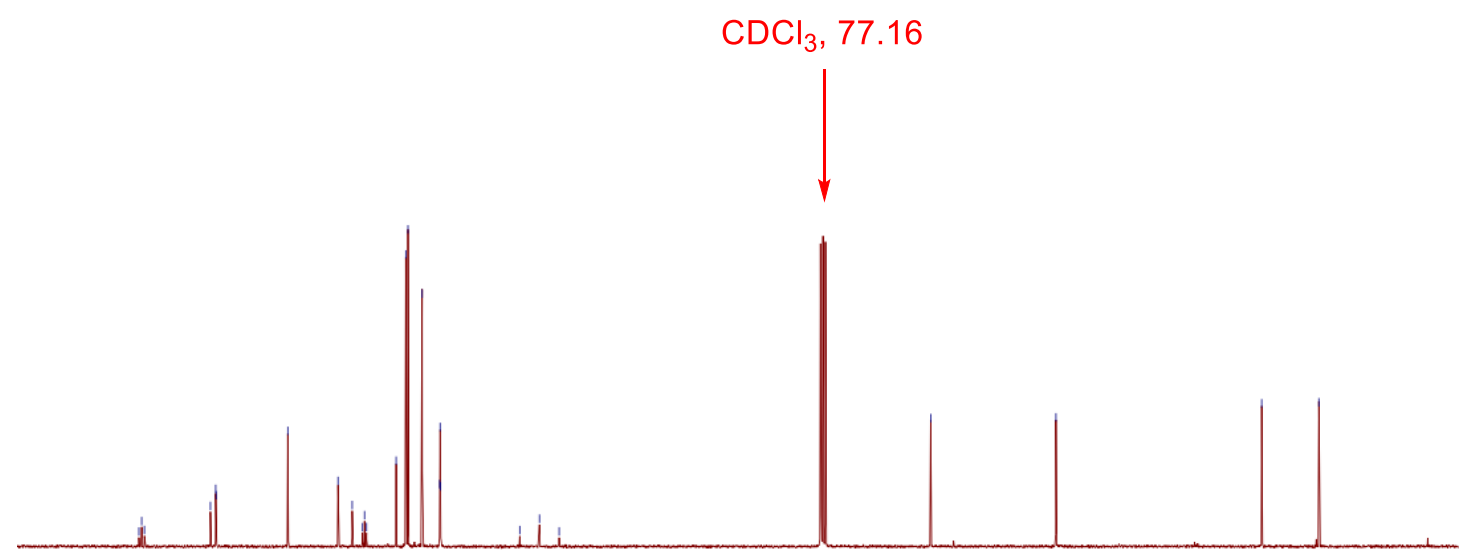

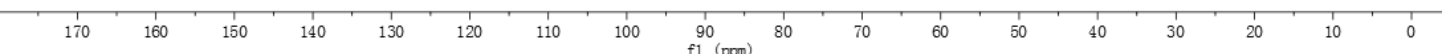


${ }^{1} \mathrm{H}$ NMR (400 MHz, $\left.\mathrm{CDCl}_{3}\right)$ of $3 \mathrm{i}$

o $\infty \infty m \infty \tilde{m}$ m の

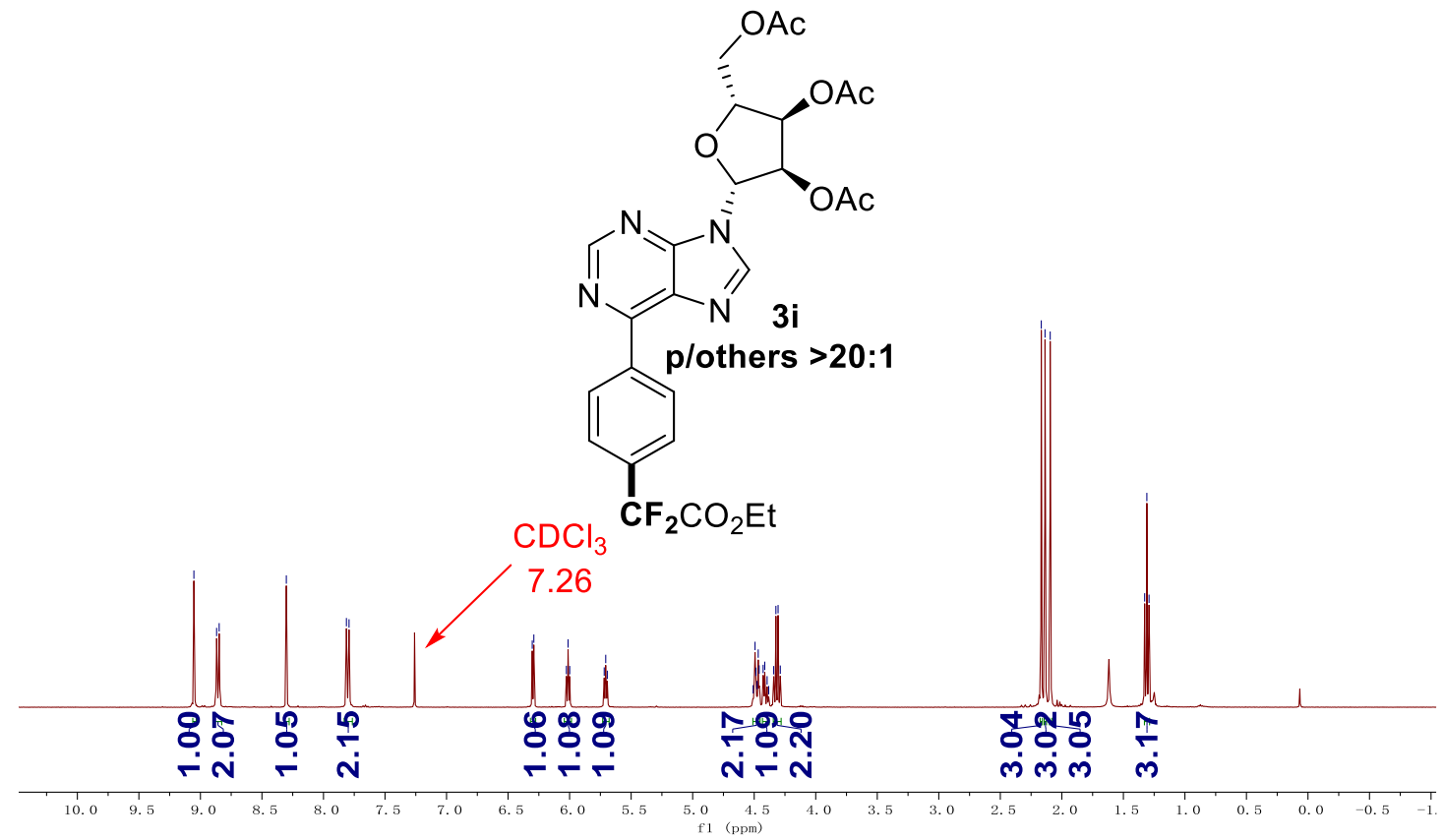

${ }^{19}$ F NMR (376 MHz, $\left.\mathrm{CDCl}_{3}\right)$ of $3 \mathrm{i}$

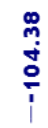

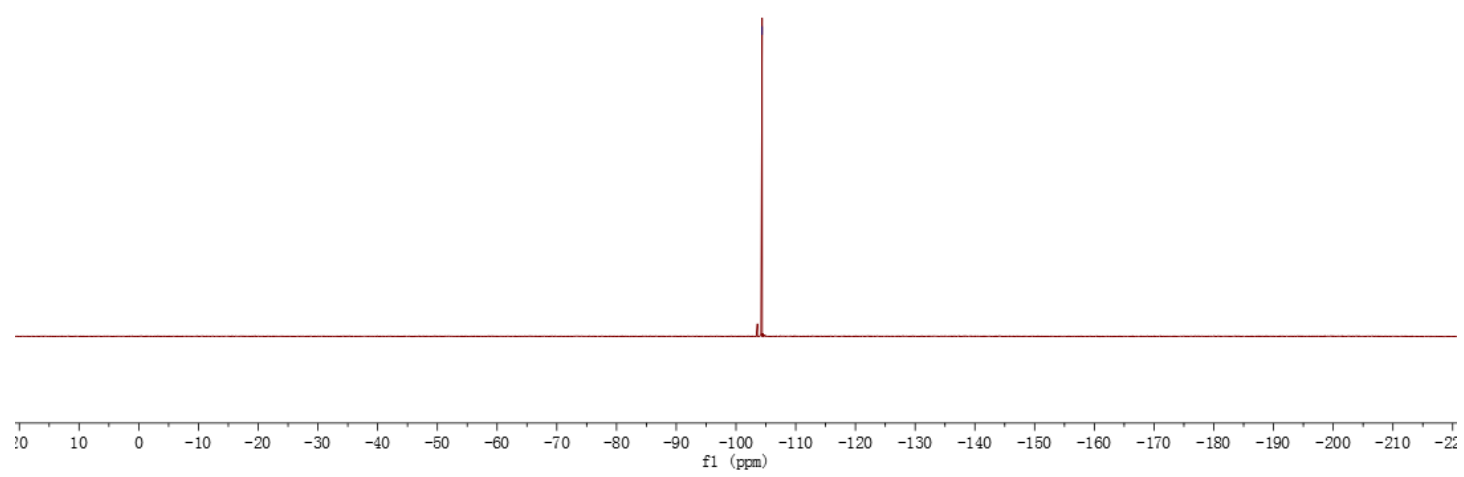


${ }^{13} \mathrm{C}$ NMR (100 MHz, $\left.\mathrm{CDCl}_{3}\right)$ of $3 \mathrm{i}$
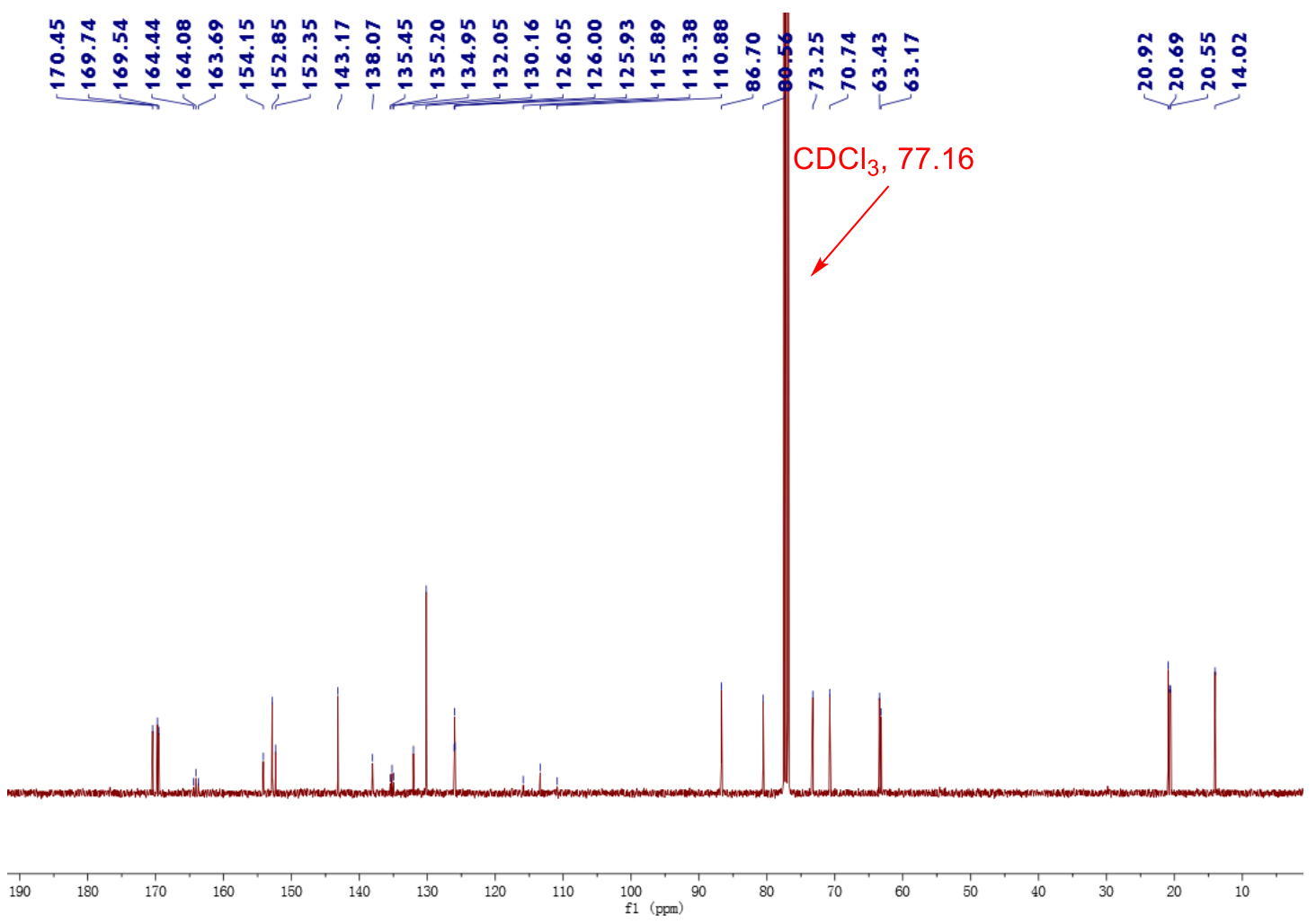

${ }^{1} \mathrm{H}$ NMR (400 MHz, $\left.\mathrm{CDCl}_{3}\right)$ of $3 \mathrm{j}$
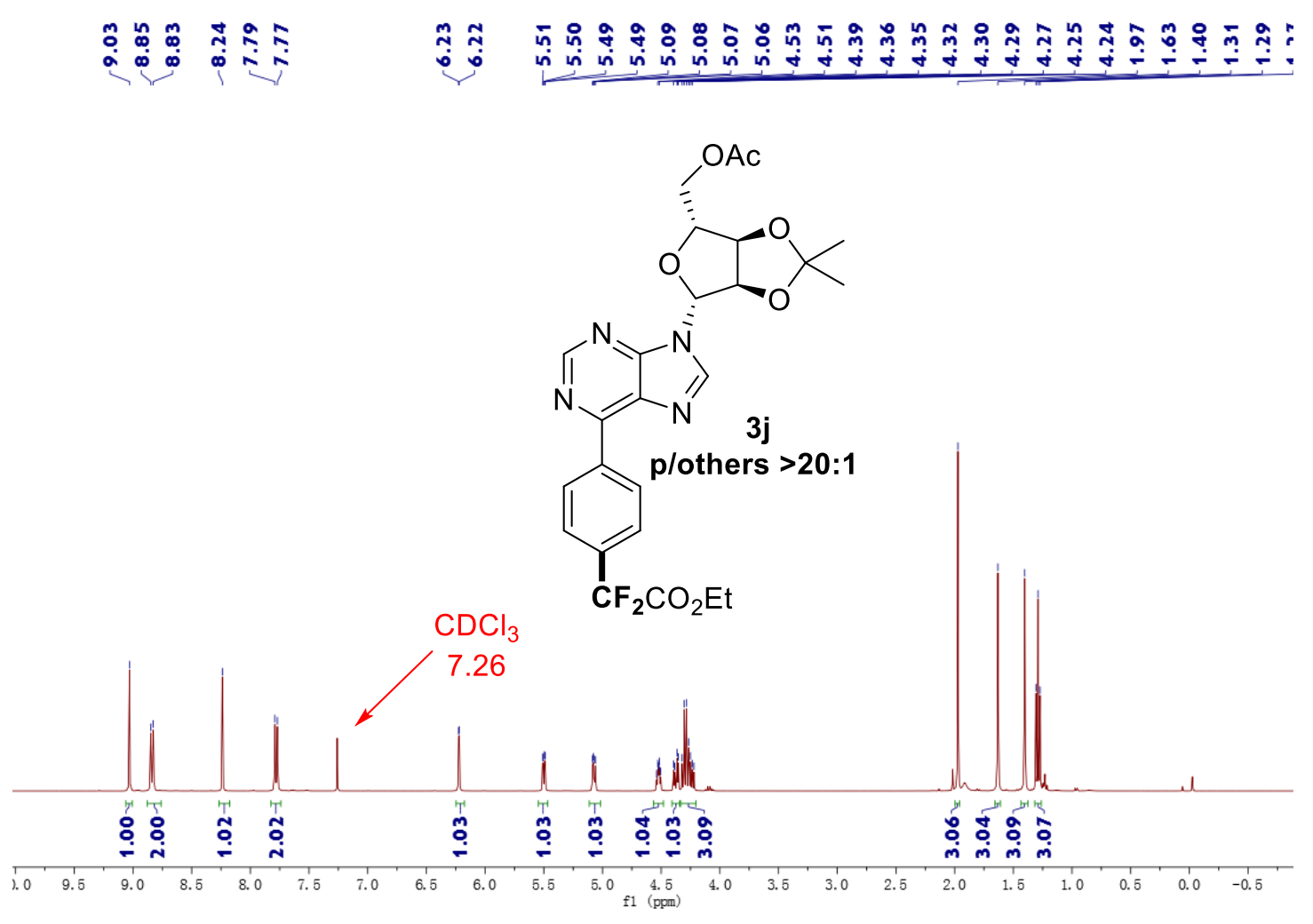

S77 
${ }^{19}$ F NMR (376 MHz, $\left.\mathrm{CDCl}_{3}\right)$ of $3 \mathrm{j}$

$$
\stackrel{\substack{m \\ \dot{0}}}{i}
$$

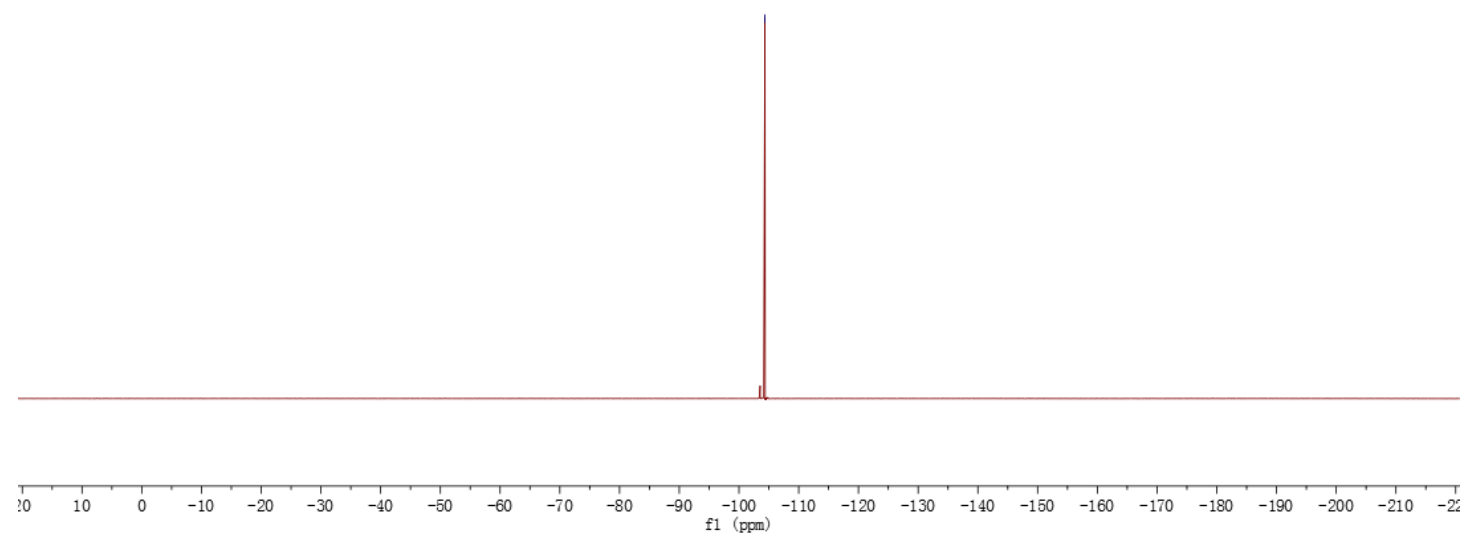

${ }^{13} \mathrm{C}$ NMR (100 $\left.\mathrm{MHz}, \mathrm{CDCl}_{3}\right)$ of $3 \mathrm{j}$

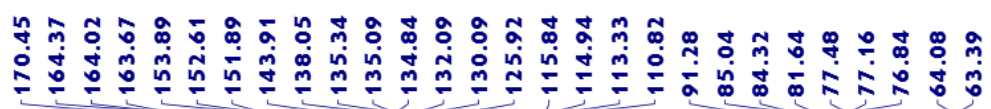

กุ

กิ ถุำ

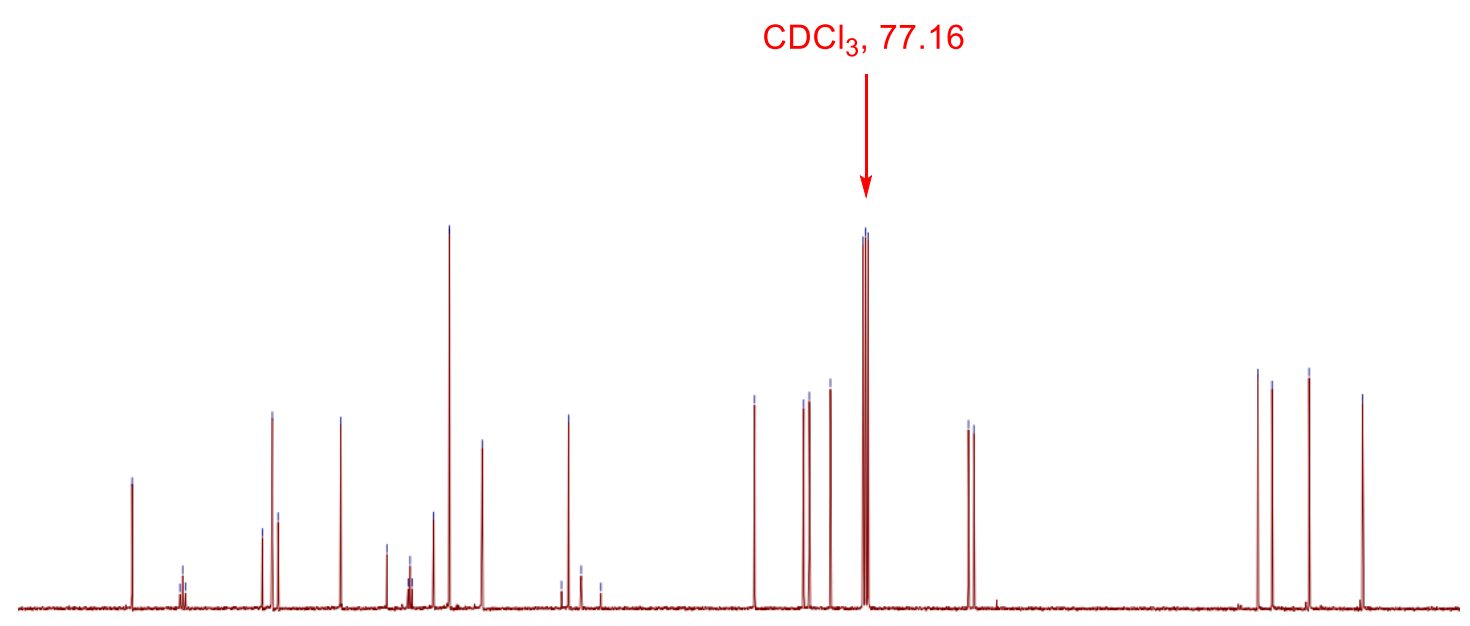

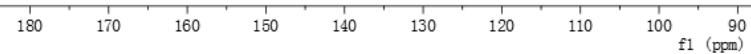


${ }^{1} \mathrm{H}$ NMR (400 MHz, $\mathrm{CDCl}_{3}$ ) of $3 \mathrm{k}$

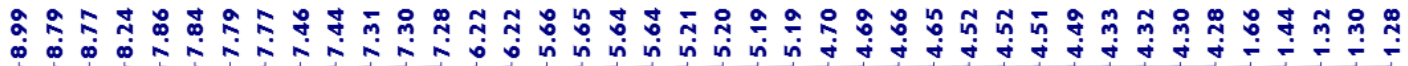
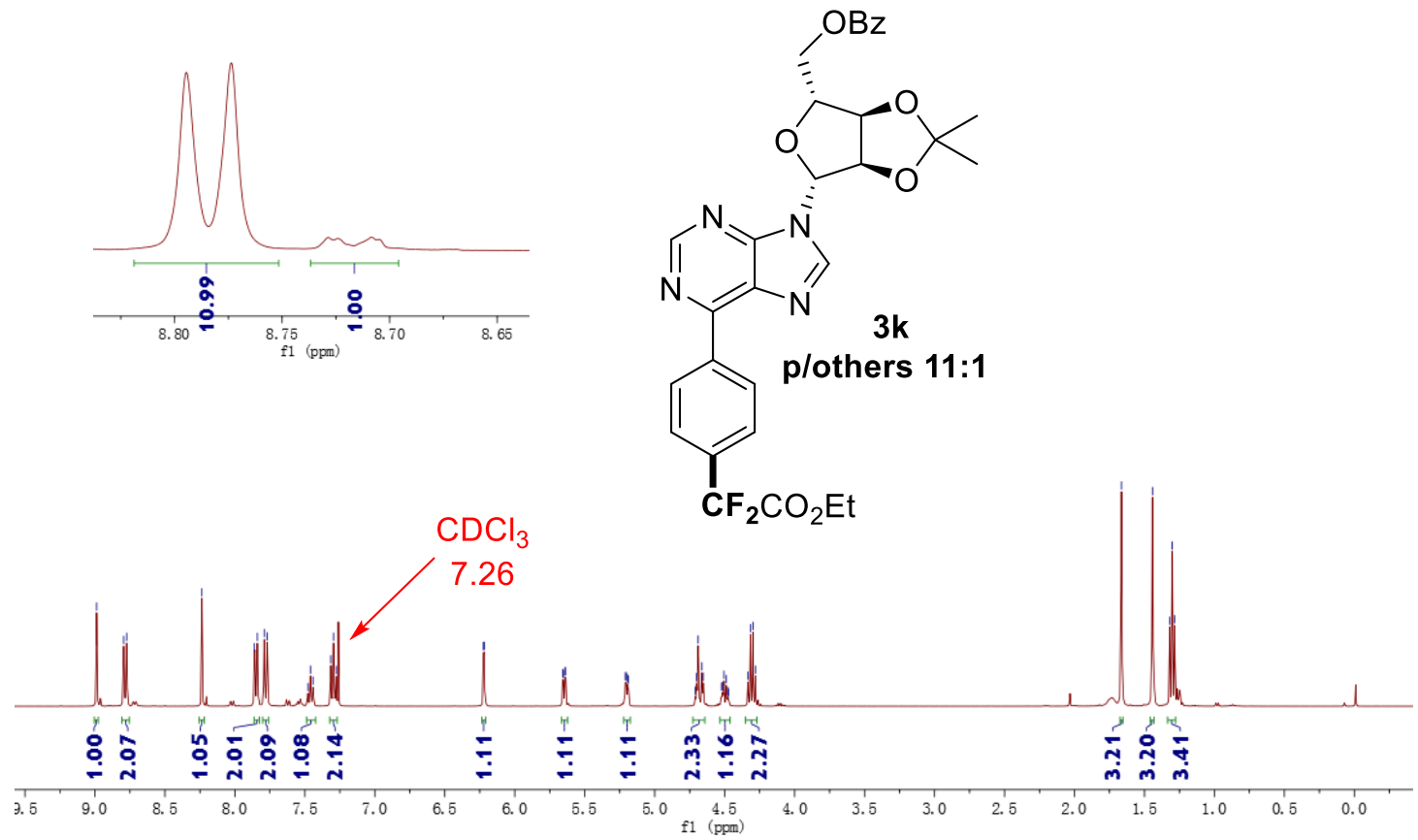

${ }^{19} \mathrm{~F}$ NMR (376 $\left.\mathrm{MHz}, \mathrm{CDCl}_{3}\right)$ of $3 \mathrm{k}$

$$
\stackrel{m}{m}
$$

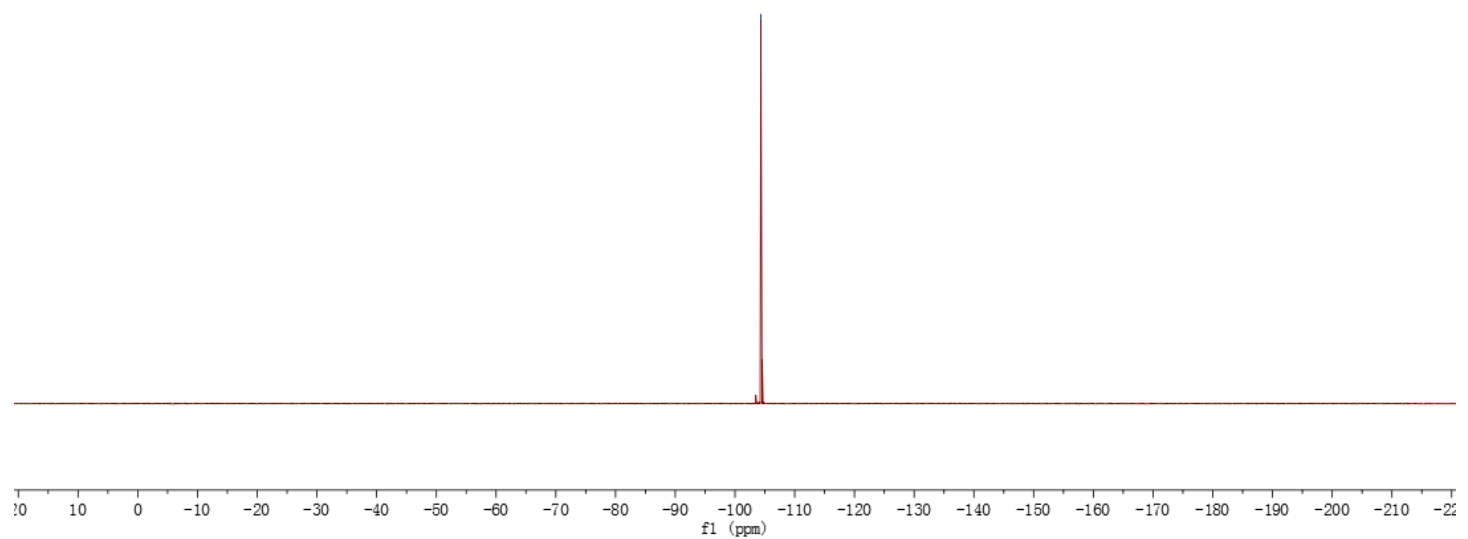


${ }^{13} \mathrm{C}$ NMR (100 $\left.\mathrm{MHz}, \mathrm{CDCl}_{3}\right)$ of $3 \mathrm{k}$

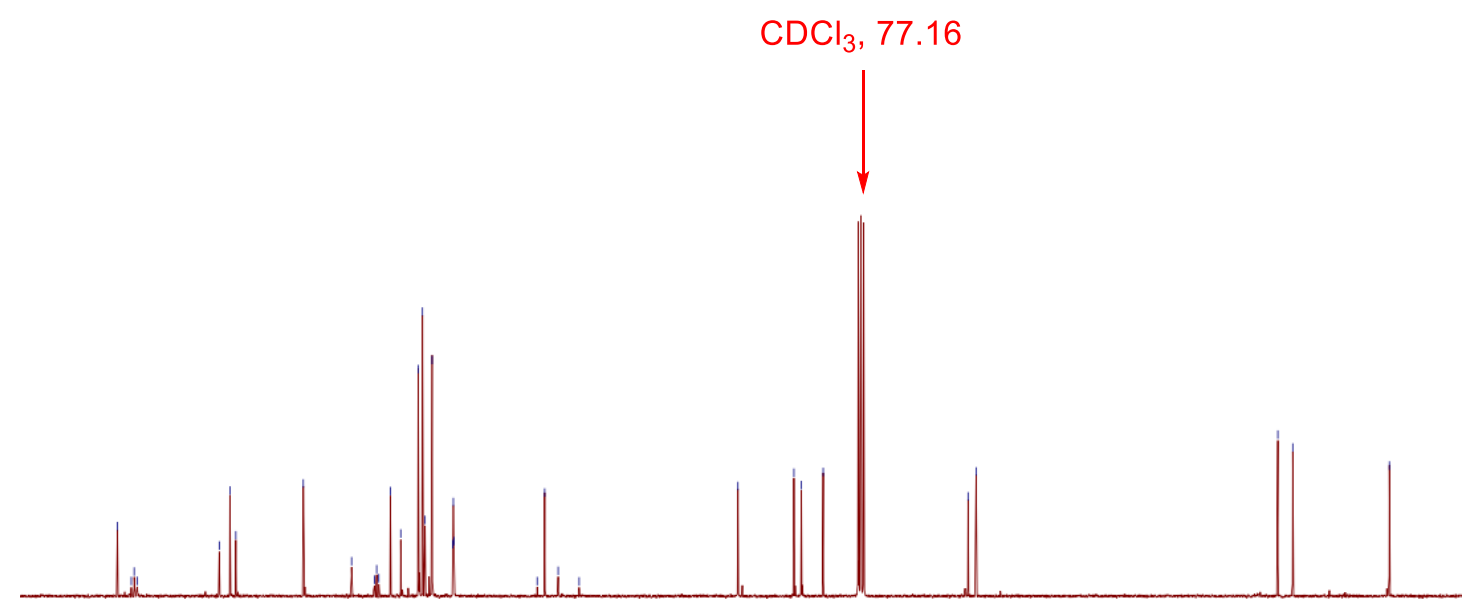

${ }^{1} \mathrm{H}$ NMR (400 MHz, $\left.\mathrm{CDCl}_{3}\right)$ of $5 \mathrm{a}$
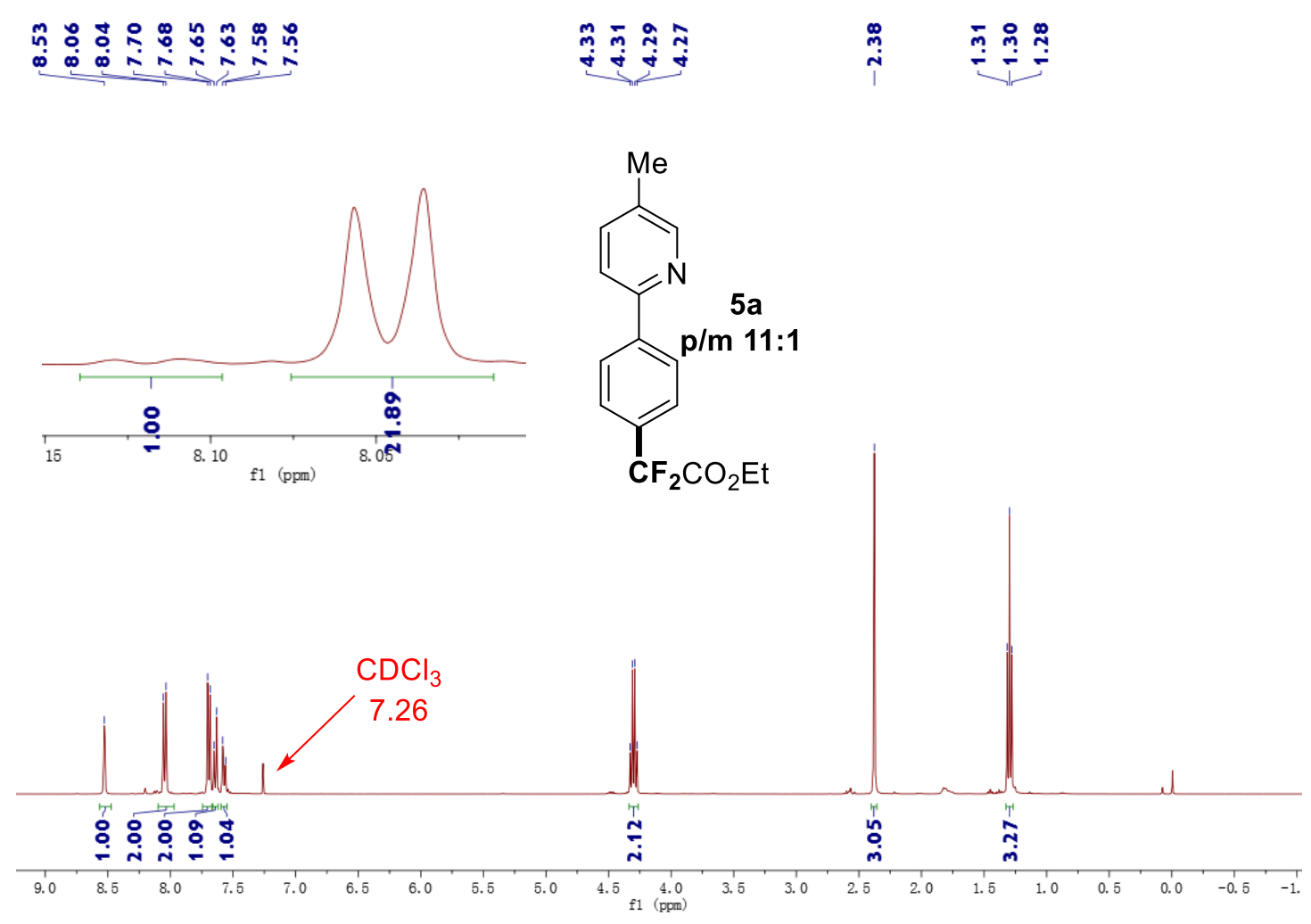
${ }^{19}$ F NMR (376 $\left.\mathrm{MHz}, \mathrm{CDCl}_{3}\right)$ of $5 \mathrm{a}$

$$
\frac{0}{\substack{n \\ 0}}
$$

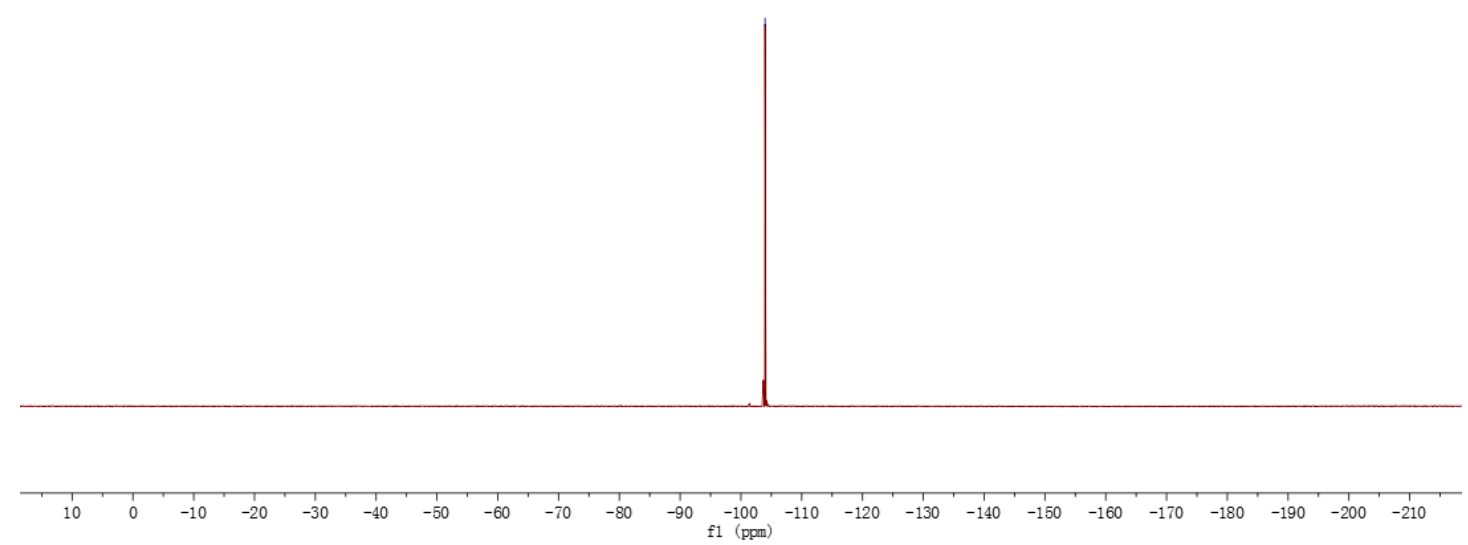

${ }^{13} \mathrm{C}$ NMR (100 MHz, $\left.\mathrm{CDCl}_{3}\right)$ of $5 \mathrm{a}$

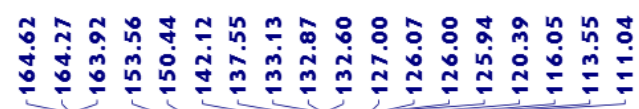

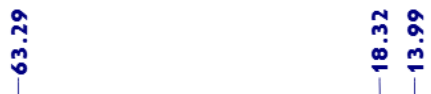
$\mathrm{CDCl}_{3}, 77.16$

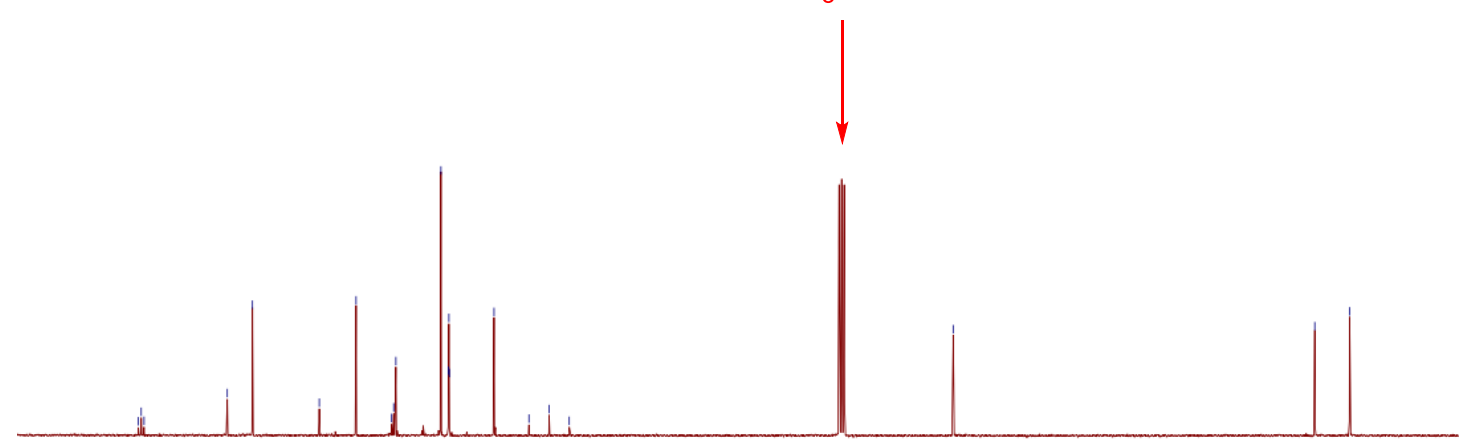

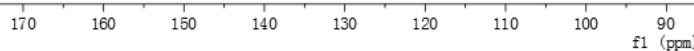


${ }^{1} \mathrm{H}$ NMR (400 MHz, $\left.\mathrm{CDCl}_{3}\right)$ of $5 \mathrm{~b}$

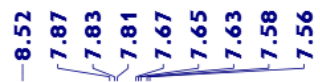

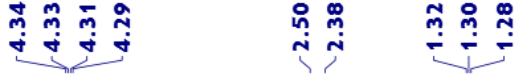
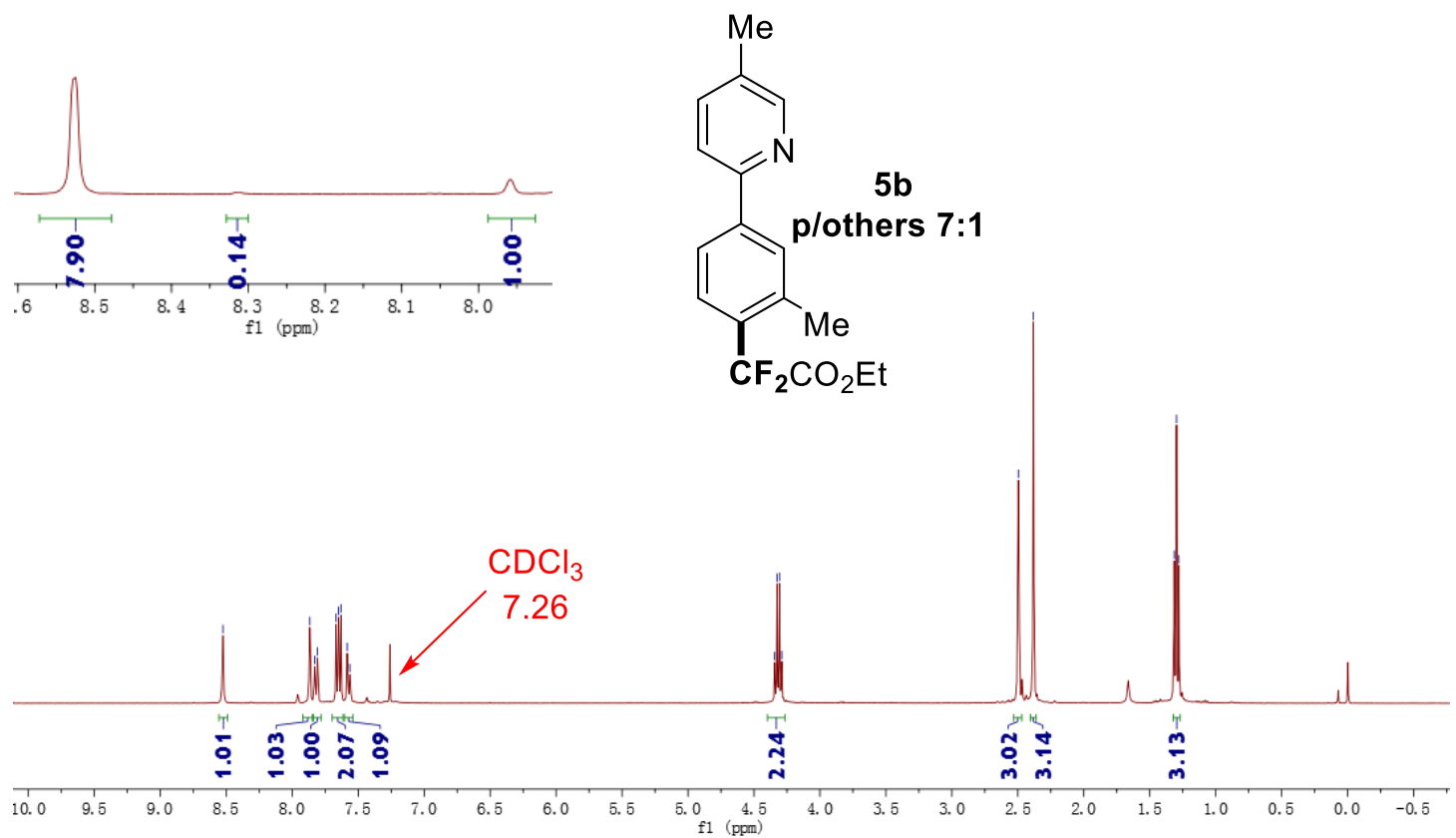

${ }^{19}$ F NMR (376 MHz, $\left.\mathrm{CDCl}_{3}\right)$ of $5 \mathrm{~b}$

$\frac{n}{\mathfrak{n}}$

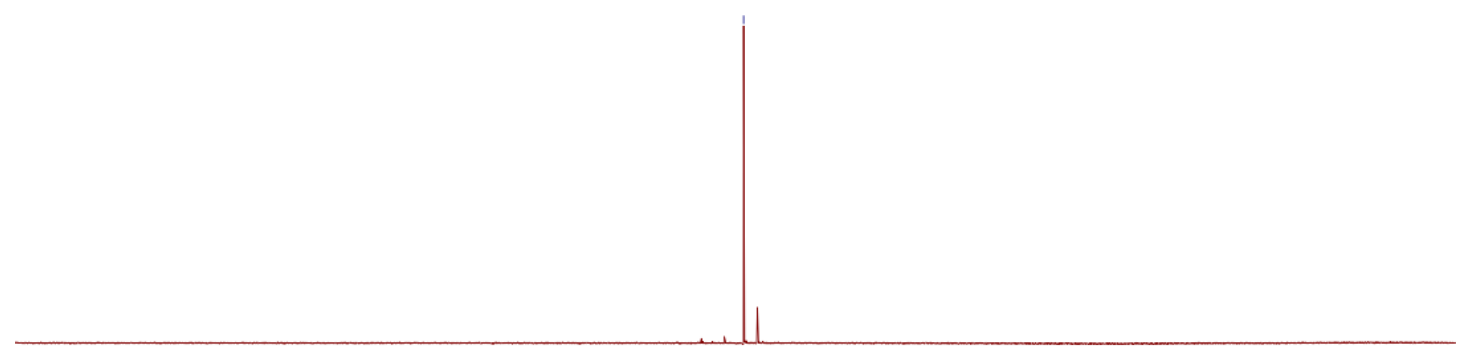

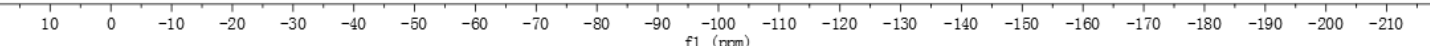


${ }^{13} \mathrm{C}$ NMR (100 MHz, $\left.\mathrm{CDCl}_{3}\right)$ of $5 \mathrm{~b}$

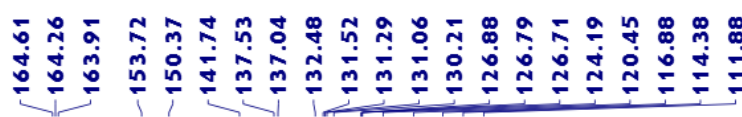

กิ่

$\stackrel{\circ}{\circ} \stackrel{0}{0}$

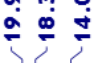

$\mathrm{CDCl}_{3}, 77.16$
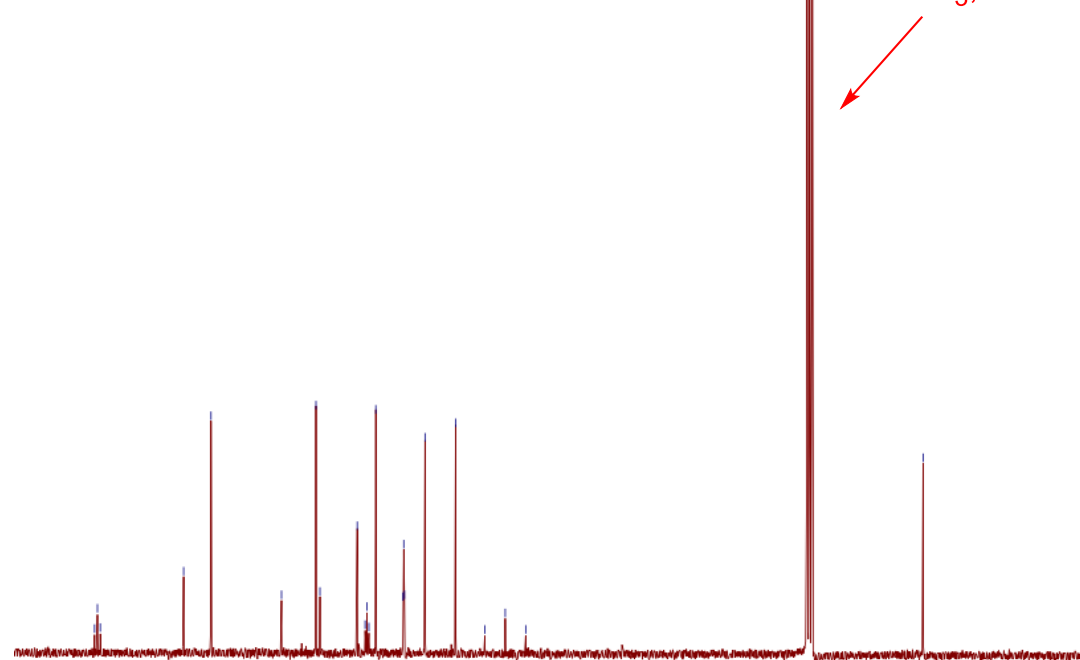

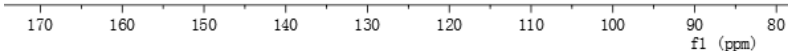

${ }^{1} \mathrm{H}$ NMR (400 MHz, $\left.\mathrm{CDCl}_{3}\right)$ of $5 \mathrm{c}$

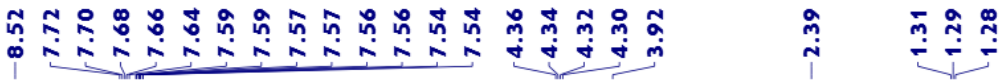

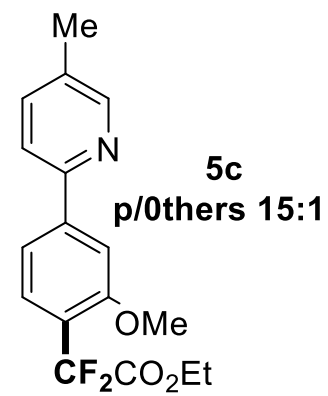

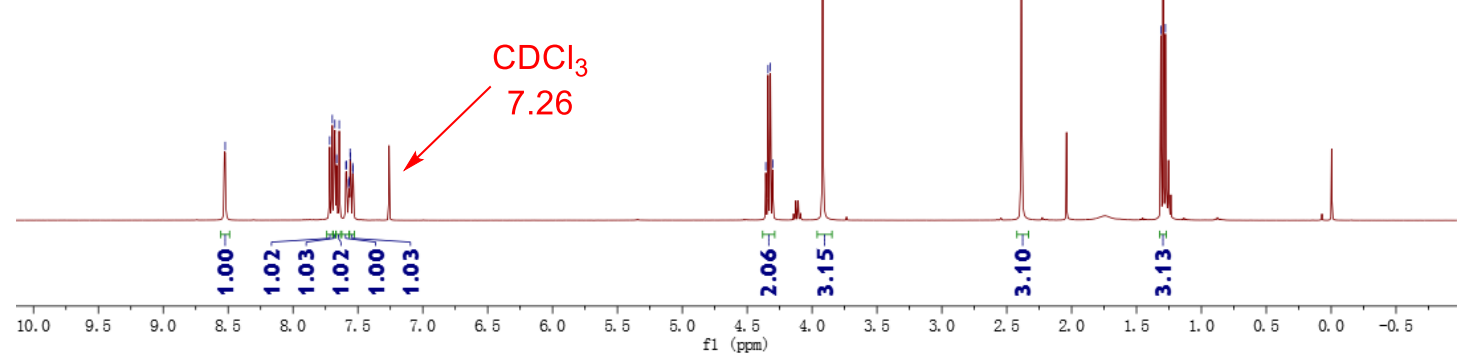


${ }^{19}$ F NMR (376 MHz, $\left.\mathrm{CDCl}_{3}\right)$ of $5 \mathrm{c}$

$n$
0
$i$
0
$i$

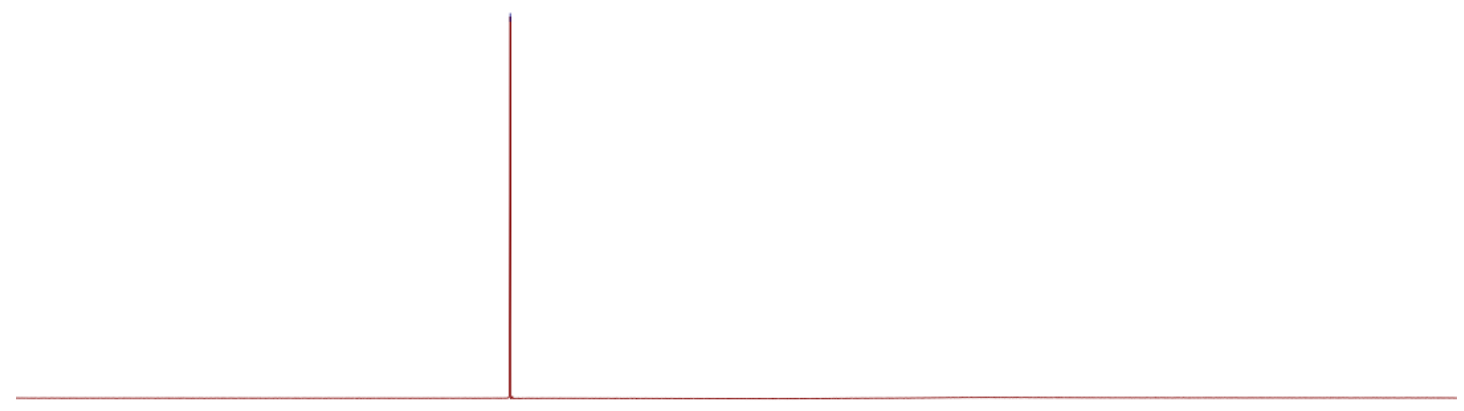

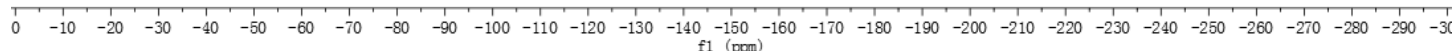

${ }^{13} \mathrm{C}$ NMR (100 MHz, $\left.\mathrm{CDCl}_{3}\right)$ of $5 \mathrm{c}$

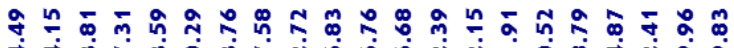

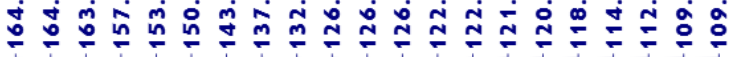

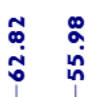

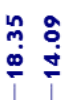

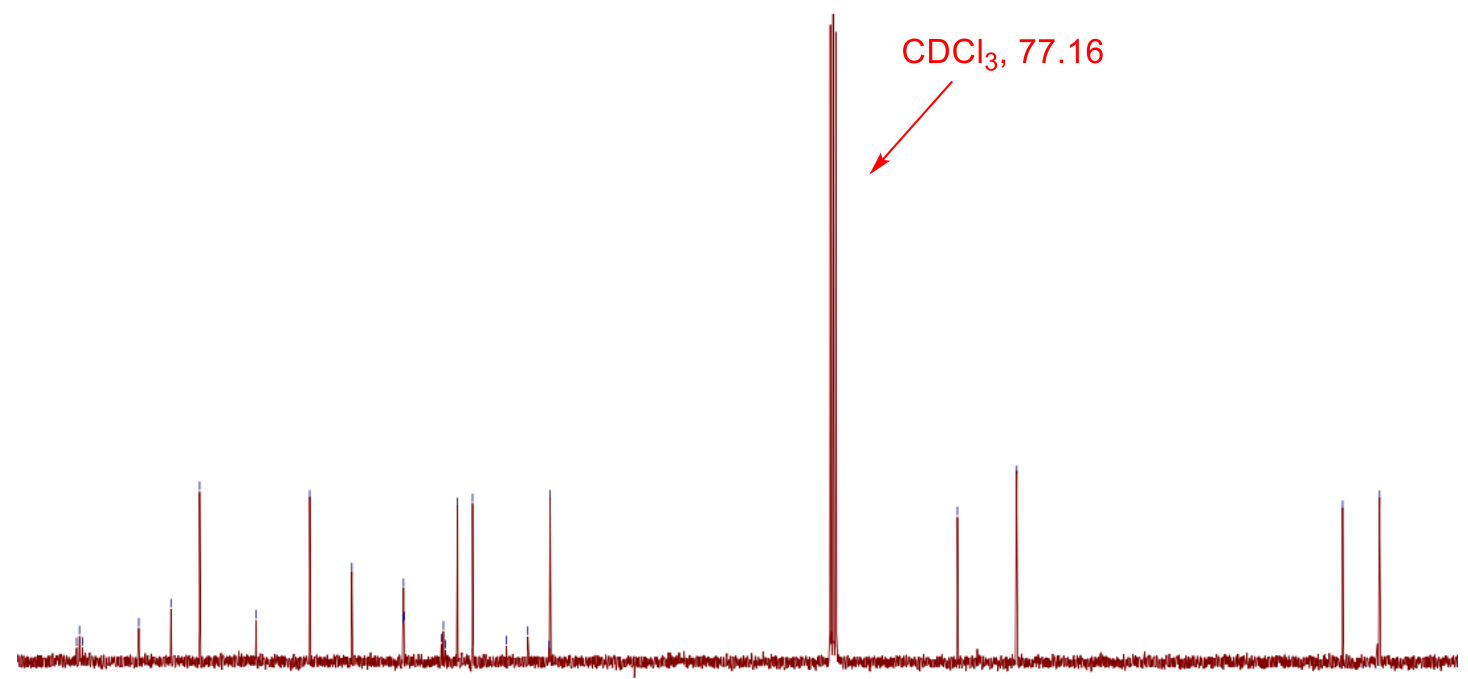

$\frac{1}{170}$

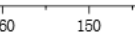

90 (ppm) 
${ }^{1} \mathrm{H}$ NMR (400 MHz, $\left.\mathrm{CDCl}_{3}\right)$ of $5 \mathrm{~d}$

䓟

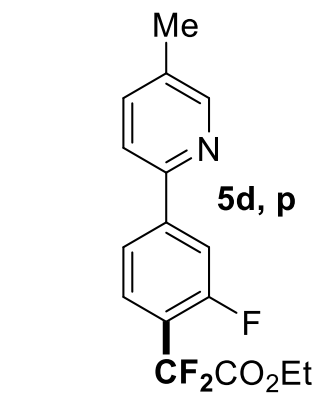

${ }^{19}$ F NMR (376 MHz, $\left.\mathrm{CDCl}_{3}\right)$ of $5 \mathrm{~d}$
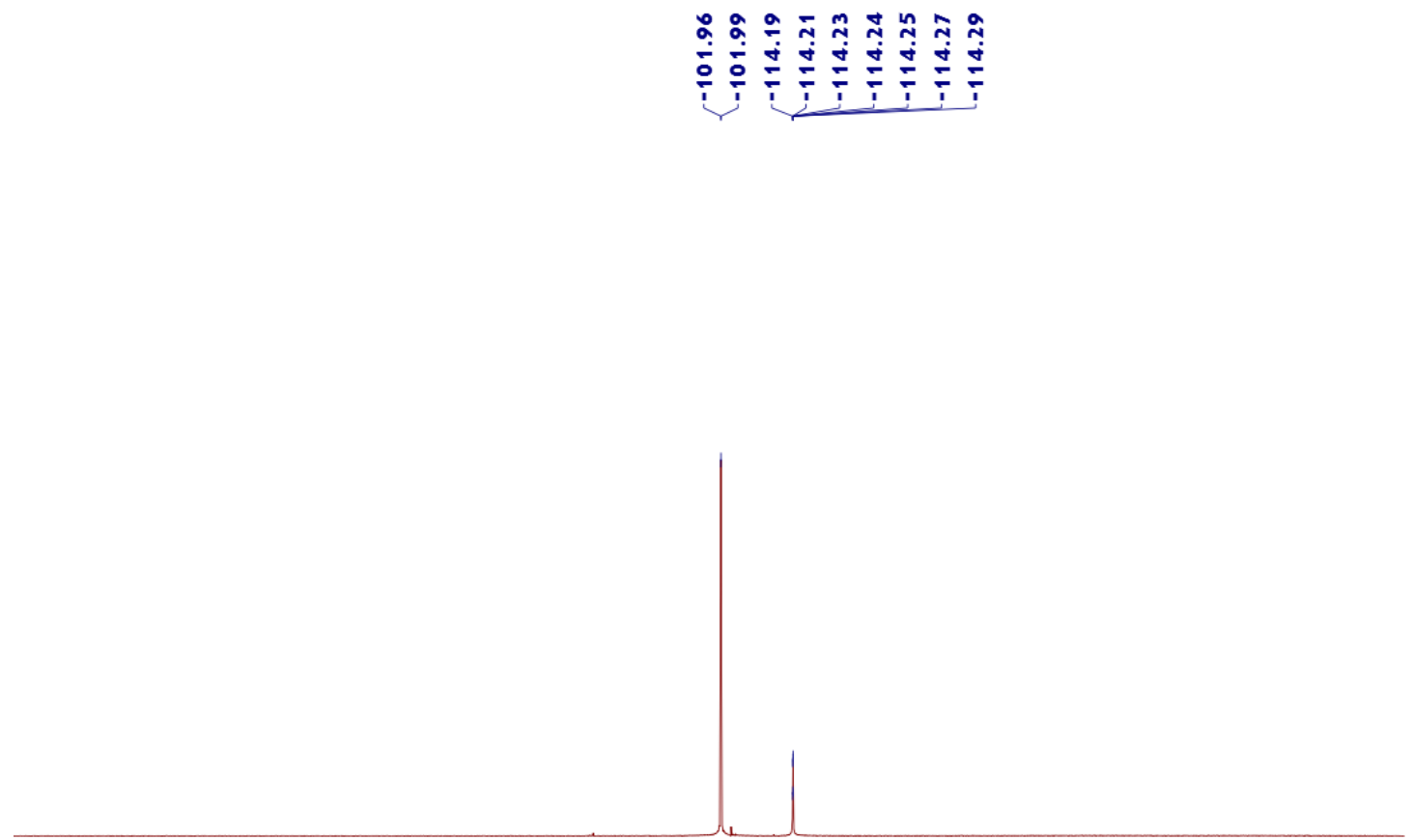

$\begin{array}{llllllllllllllllllllllllll}10 & 0 & -10 & -20 & -30 & -40 & -50 & -60 & -70 & -80 & -90 & -100 & -110 & -120 & -130 & -140 & -150 & -160 & -170 & -180 & -190 & -200 & -210\end{array}$ 


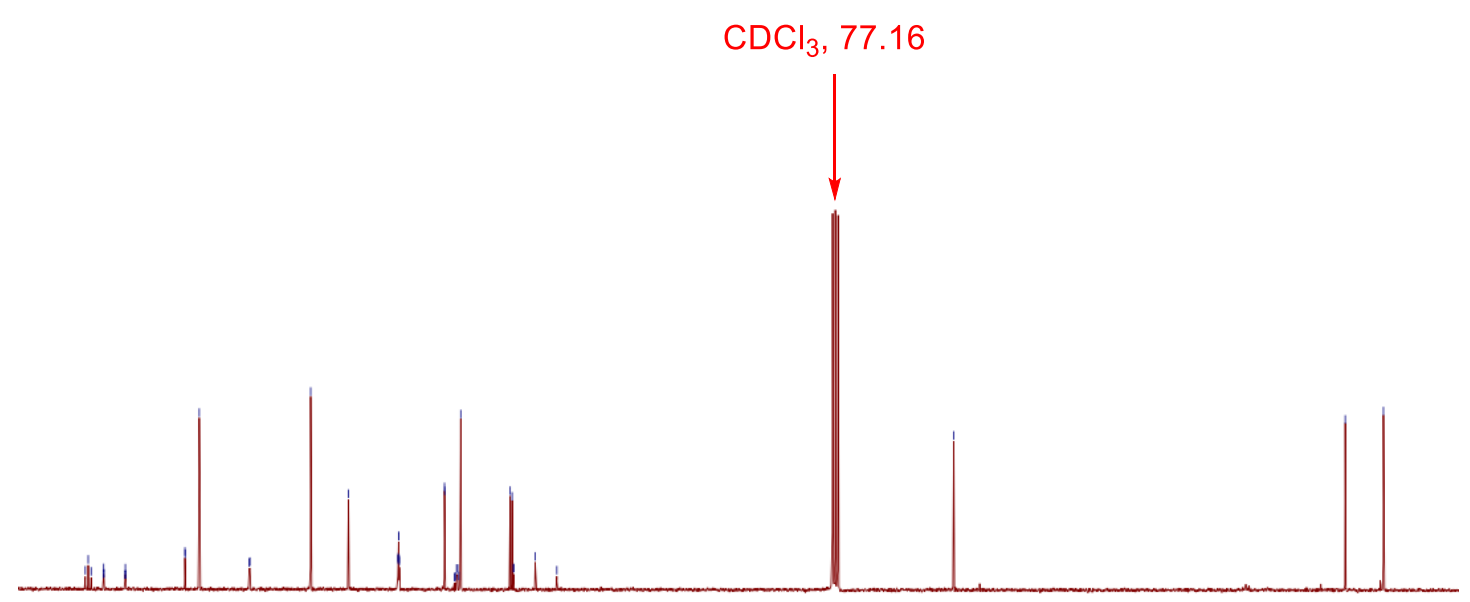

$\underset{170}{1}$

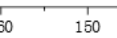

140

130

$f^{90}(\mathrm{pom})$

${ }^{1} \mathrm{H}$ NMR (400 MHz, $\left.\mathrm{CDCl}_{3}\right)$ of $5 \mathrm{e}$

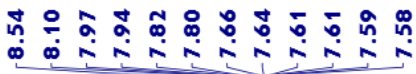

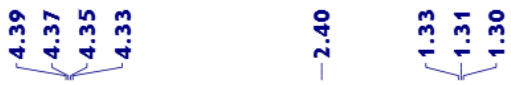

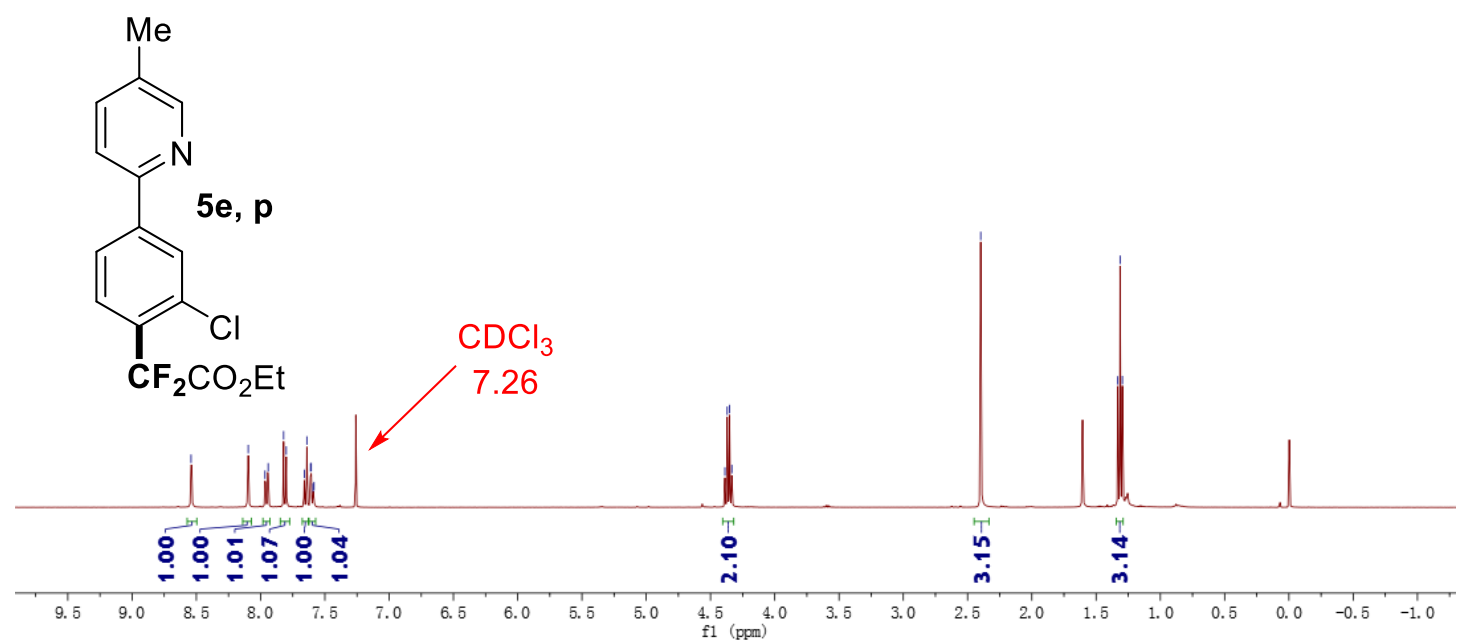


${ }^{19}$ F NMR (376 MHz, $\left.\mathrm{CDCl}_{3}\right)$ of $5 \mathrm{e}$

$$
\frac{i}{i}
$$

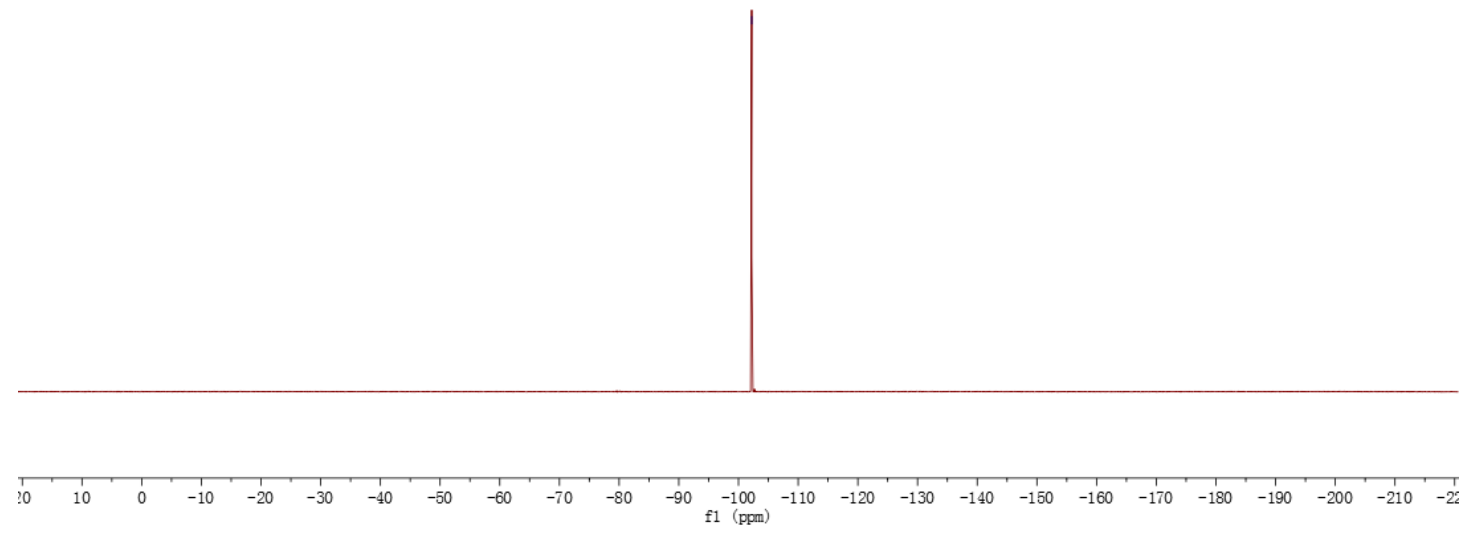

${ }^{13} \mathrm{C}$ NMR (100 MHz, $\left.\mathrm{CDCl}_{3}\right)$ of $5 \mathrm{e}$

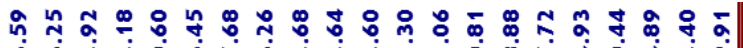

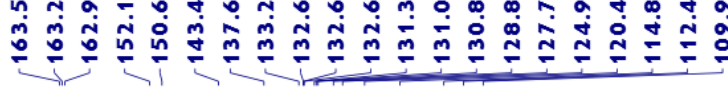

$\underset{i n}{i}$

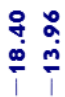

$\mathrm{CDCl}_{3}, 77.16$

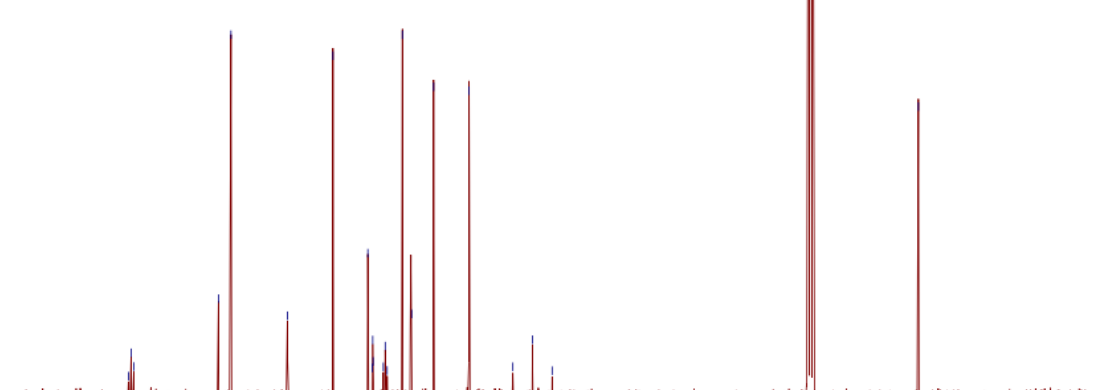

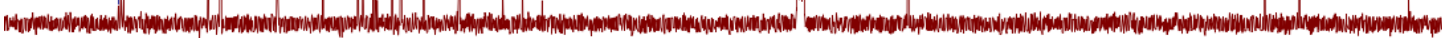

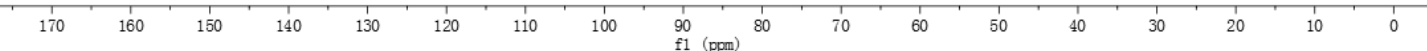


${ }^{1} \mathrm{H}$ NMR (400 MHz, $\left.\mathrm{CDCl}_{3}\right)$ of $5 f$

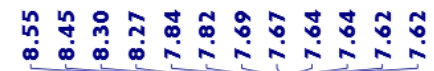

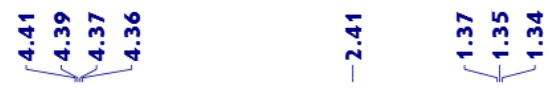

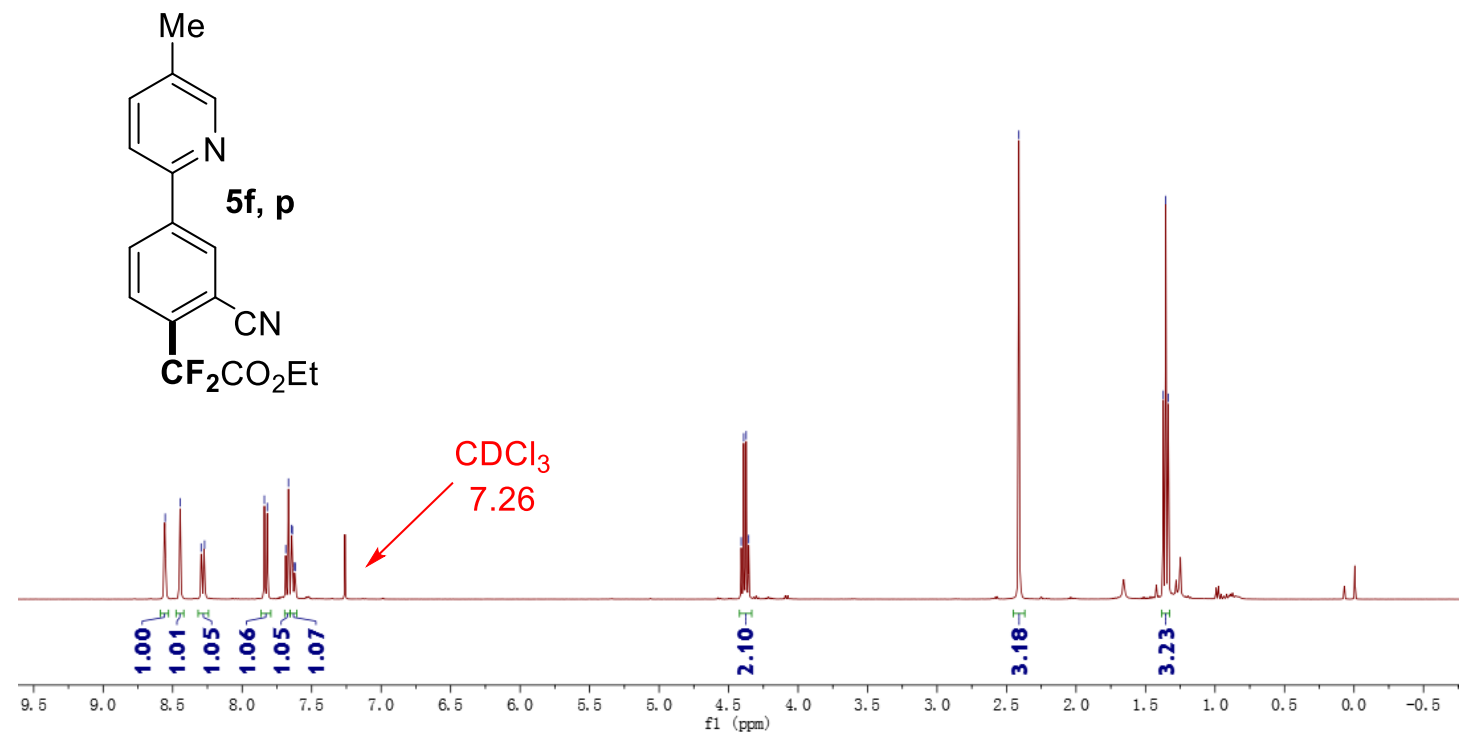

${ }^{19}$ F NMR (376 MHz, $\left.\mathrm{CDCl}_{3}\right)$ of $5 f$

$$
\frac{m}{\stackrel{m}{i}}
$$

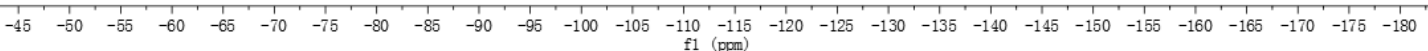




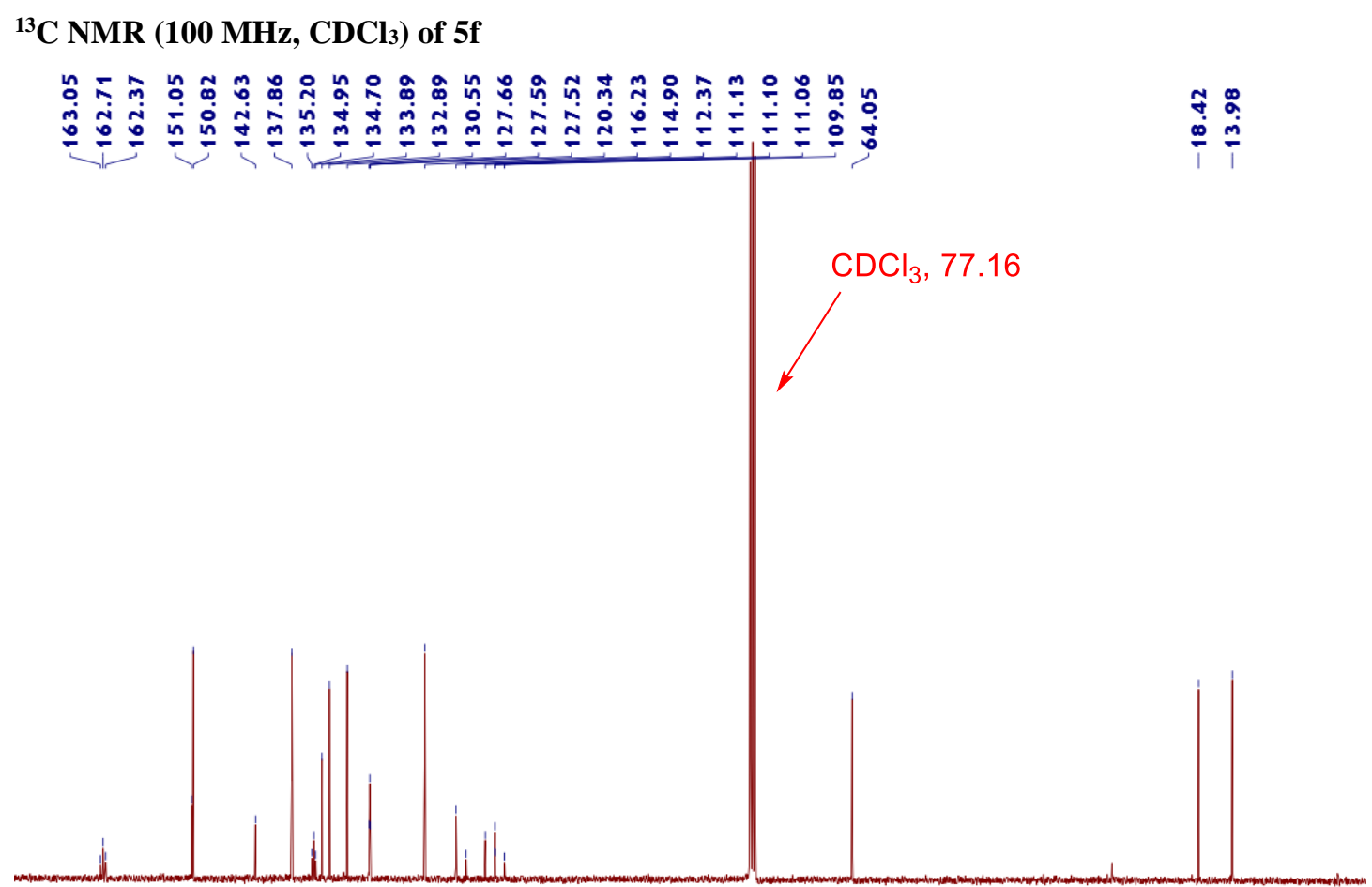

${ }^{1} \mathrm{H}$ NMR (400 MHz, $\left.\mathrm{CDCl}_{3}\right)$ of $5 \mathrm{~g}$

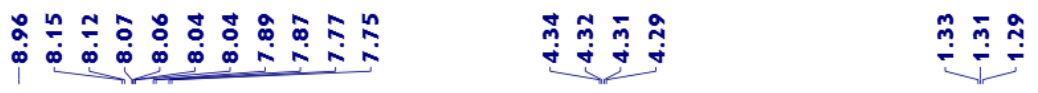
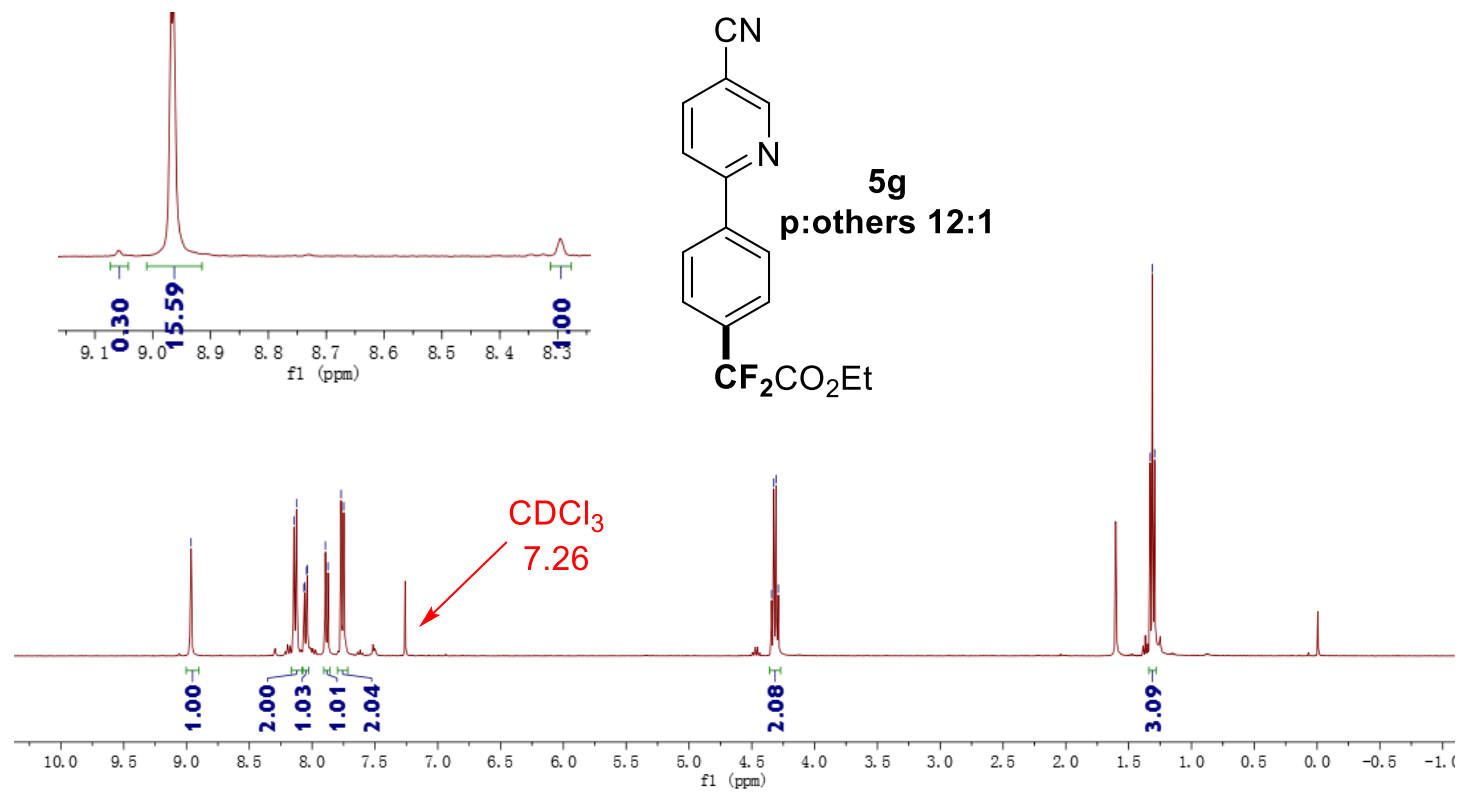
${ }^{19} \mathrm{~F}$ NMR (376 $\left.\mathrm{MHz}, \mathrm{CDCl}_{3}\right)$ of $5 \mathrm{~g}$

กั่

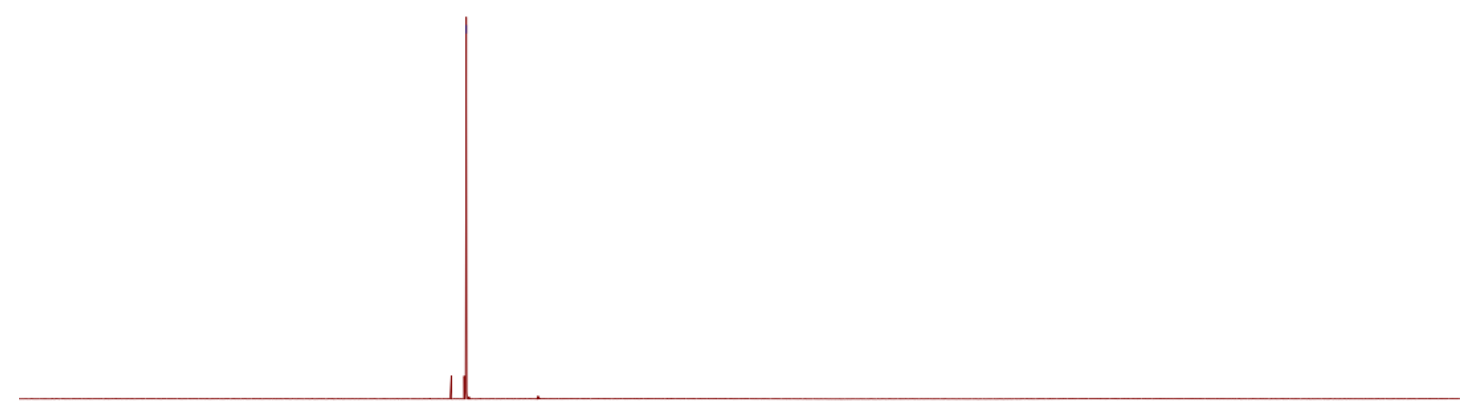

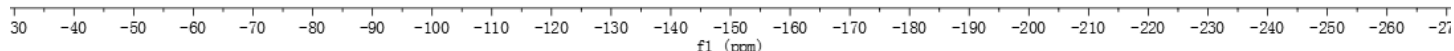

${ }^{13} \mathrm{C}$ NMR (100 MHz, $\left.\mathrm{CDCl}_{3}\right)$ of $5 \mathrm{~g}$

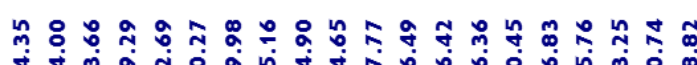

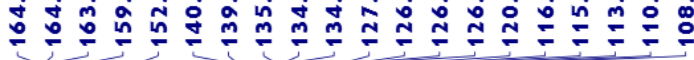

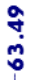

$\underset{\dot{j}}{\dot{+}}$

$\mathrm{CDCl}_{3}, 77.16$
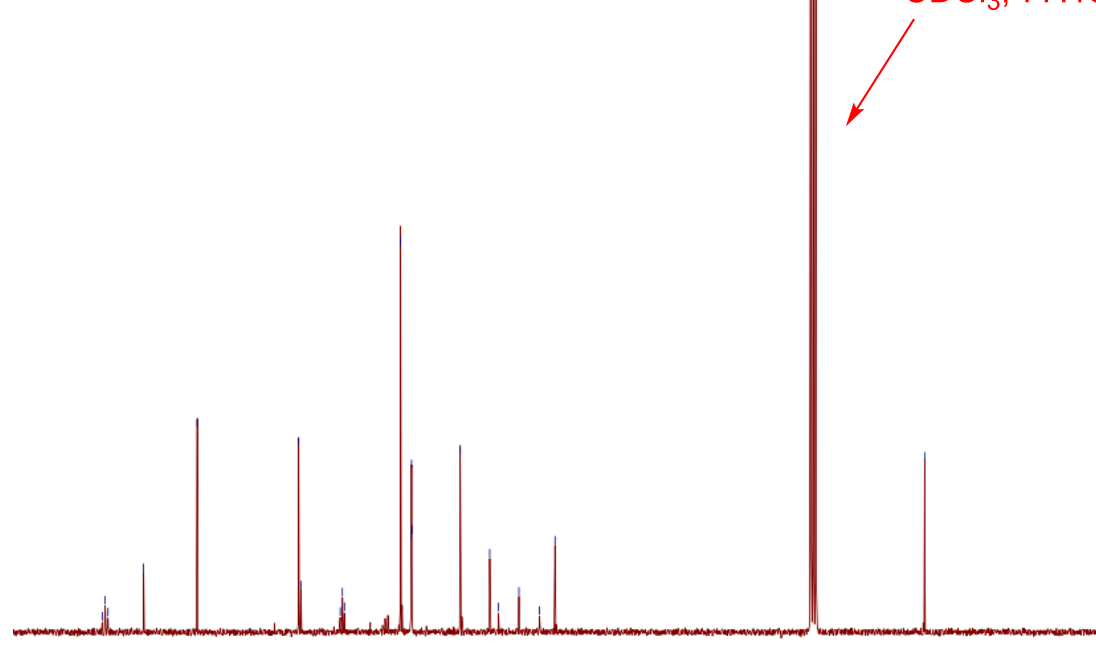

$170 \quad 160$

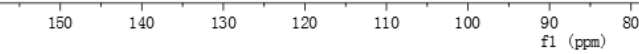

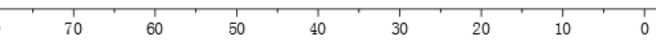


${ }^{1} \mathrm{H}$ NMR (400 MHz, CDCl 3$)$ of $5 \mathrm{~h}$

宓

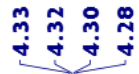

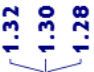<smiles>Clc1ccccn1</smiles>

$5 \mathrm{~h}$

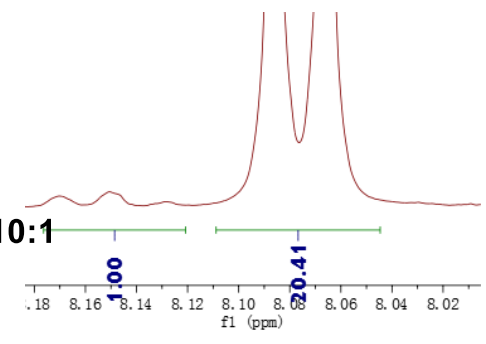

$\mathrm{CF}_{2} \mathrm{CO}_{2} \mathrm{Et}$

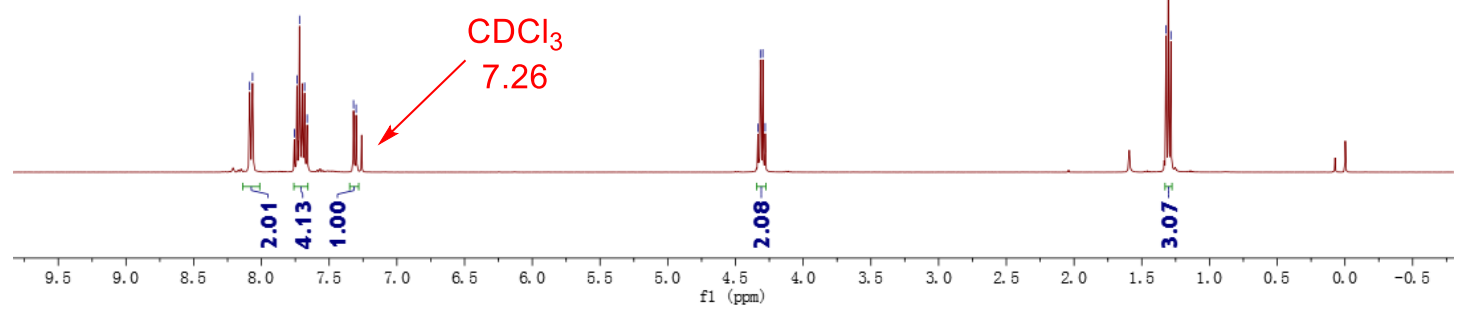

${ }^{19}$ F NMR (376 MHz, $\left.\mathrm{CDCl}_{3}\right)$ of $5 \mathrm{~h}$

$$
\text { ํํํ }
$$

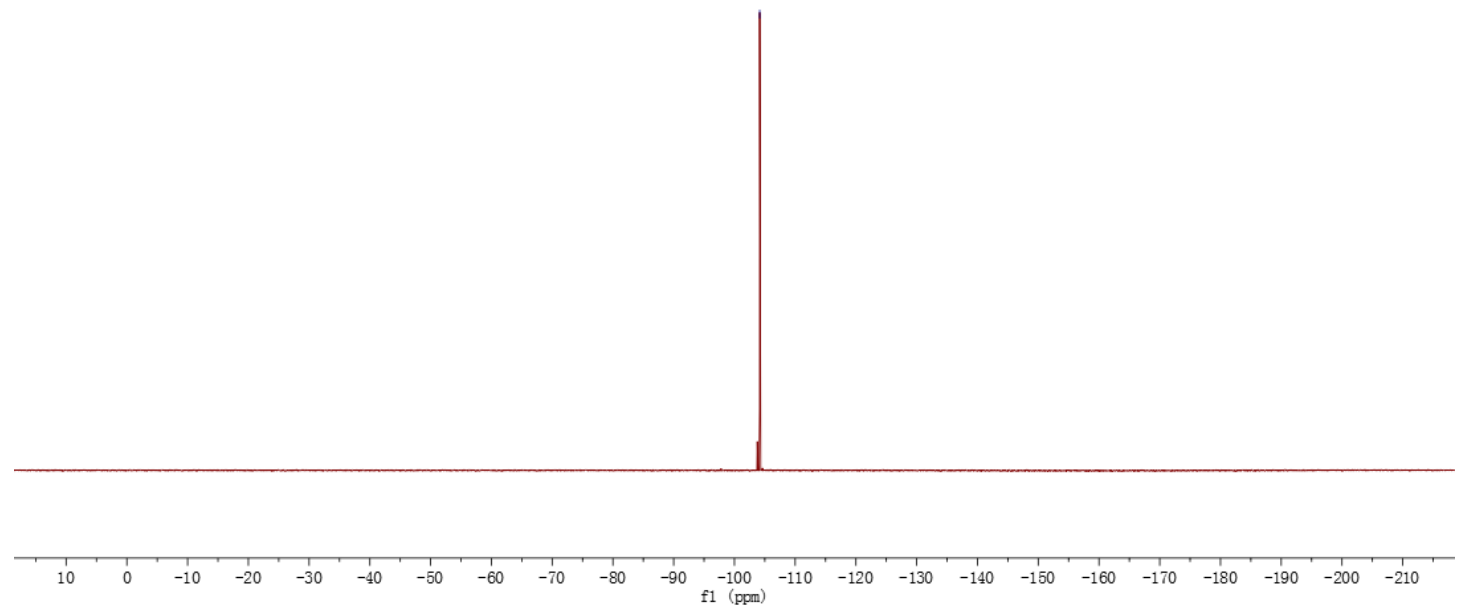


${ }^{13} \mathrm{C}$ NMR (100 $\left.\mathrm{MHz}, \mathrm{CDCl}_{3}\right)$ of $5 \mathrm{~h}$

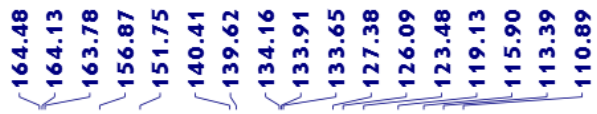

$\mathrm{CDCl}_{3}, 77.16$

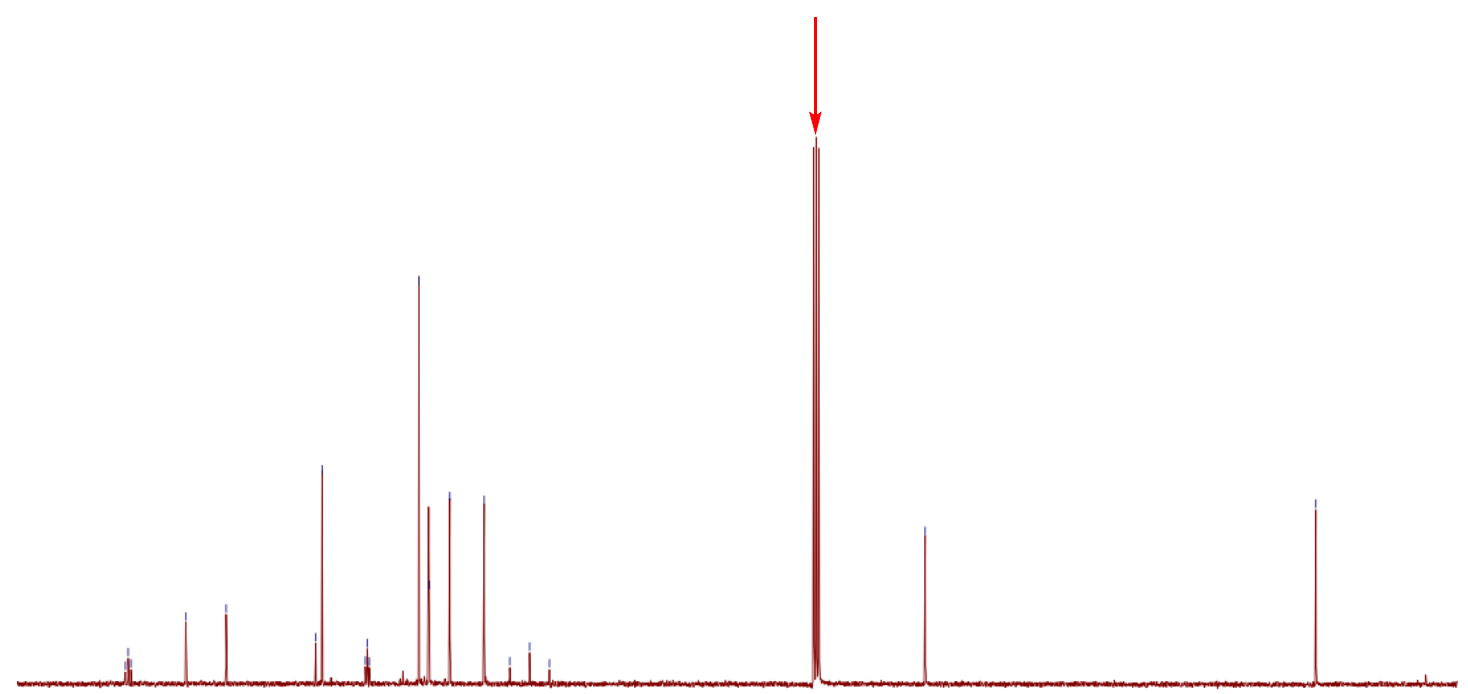

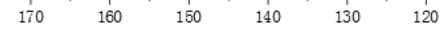

${ }^{1} \mathrm{H}$ NMR $\left(400 \mathrm{MHz}, \mathrm{CDCl}_{3}\right)$ of $5 \mathrm{i}$

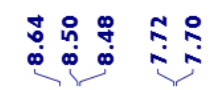

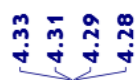

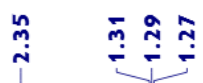

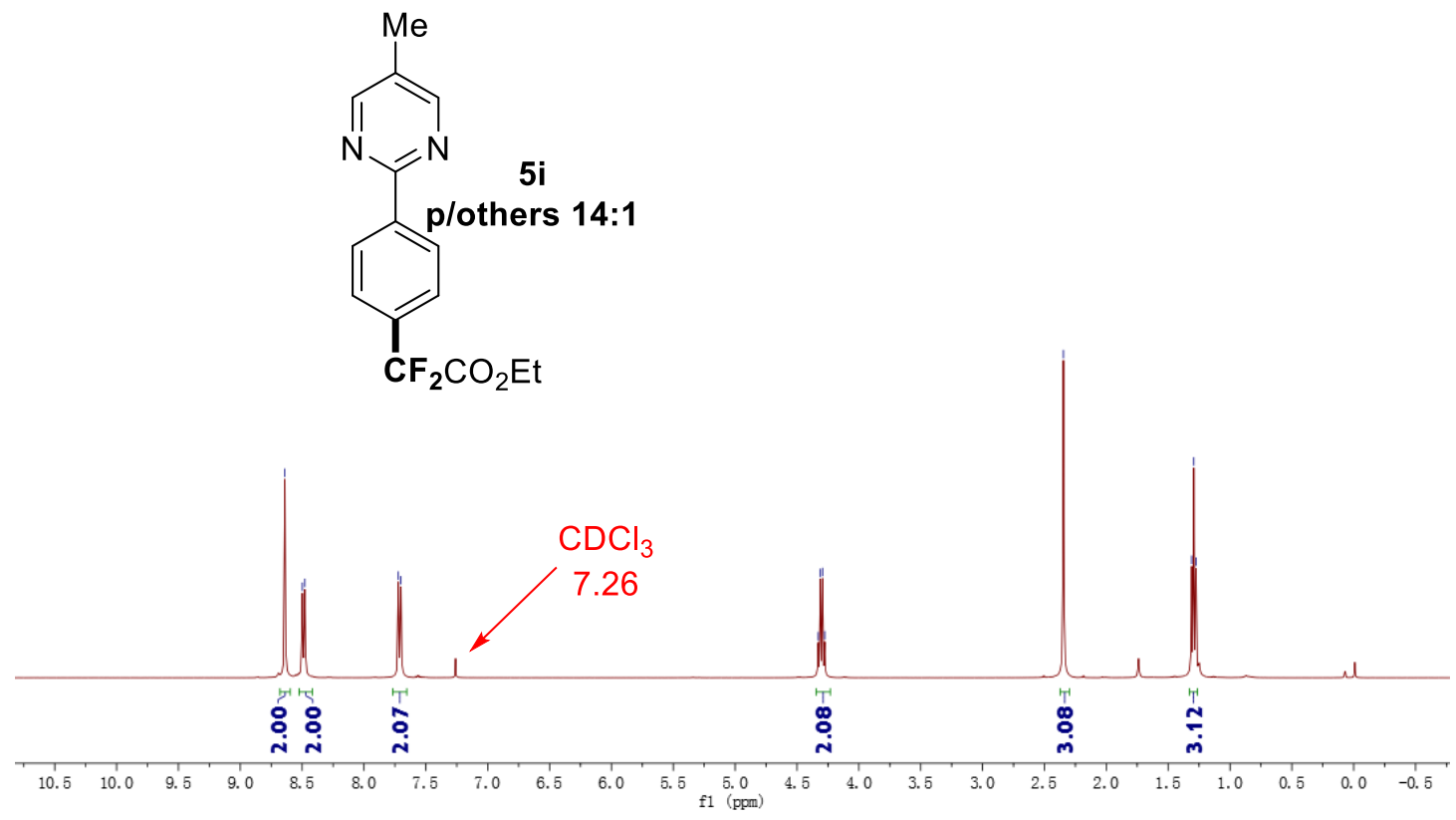


${ }^{19}$ F NMR (376 MHz, $\left.\mathrm{CDCl}_{3}\right)$ of $5 \mathrm{i}$

$$
\frac{\check{j}}{\grave{i}}
$$

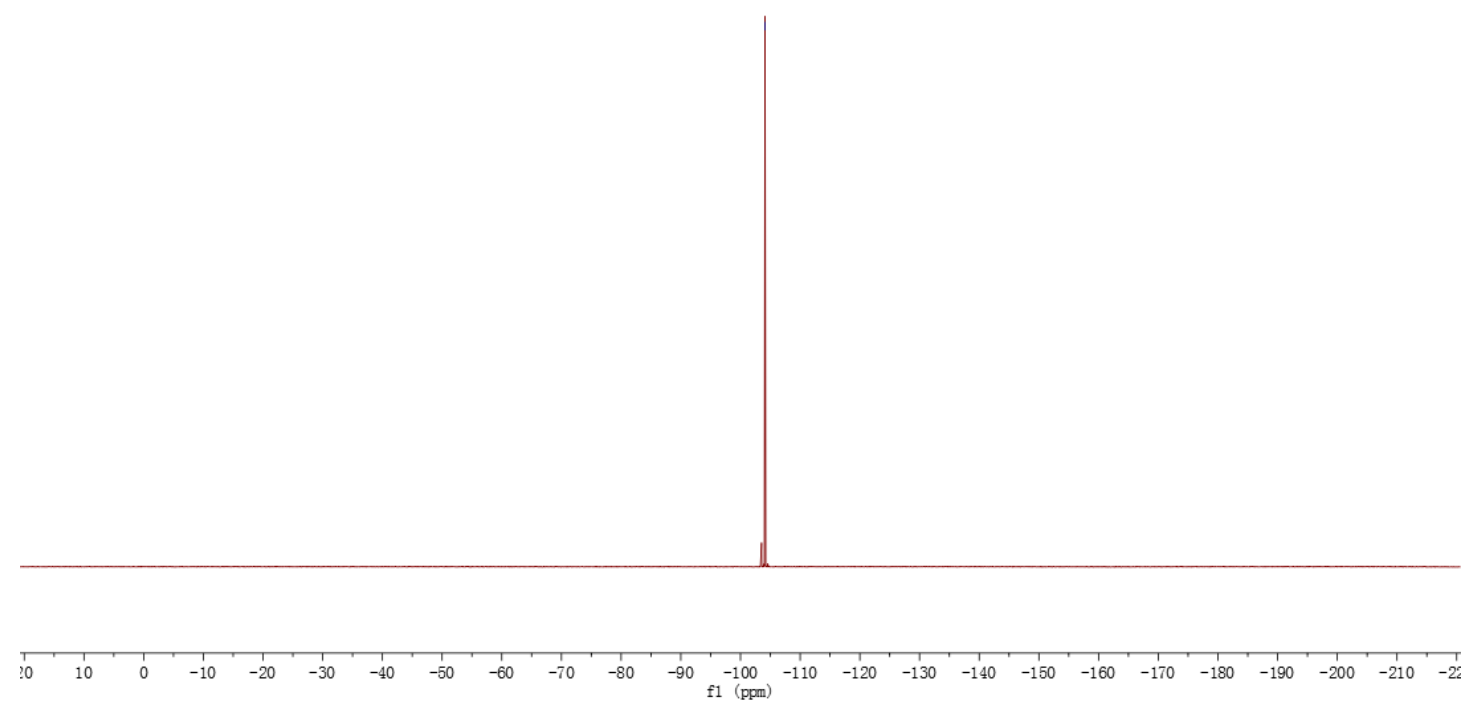

${ }^{13} \mathrm{C}$ NMR (100 MHz, $\left.\mathrm{CDCl}_{3}\right)$ of $5 \mathrm{i}$

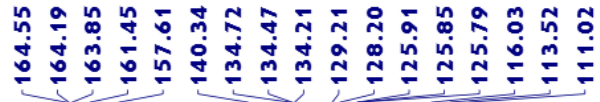

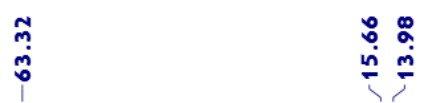

$\mathrm{CDCl}_{3}, 77.16$

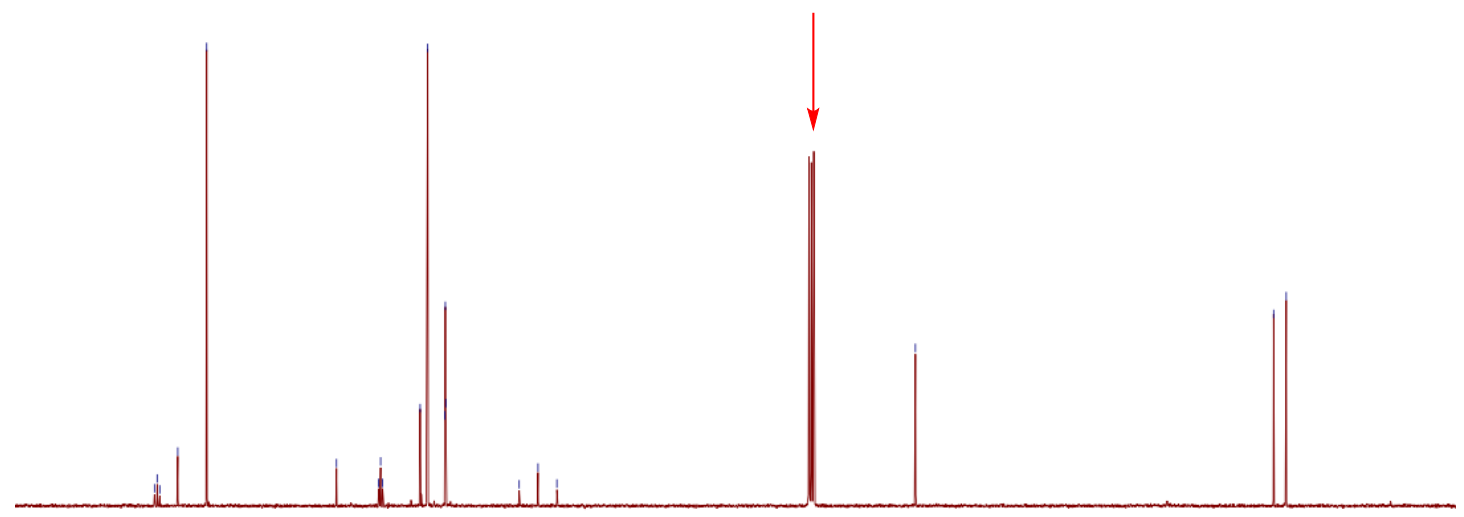

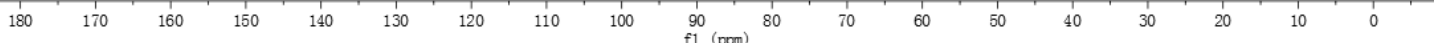


${ }^{1} \mathrm{H}$ NMR (400 MHz, $\left.\mathrm{CDCl}_{3}\right)$ of $5 \mathbf{j}$

象突
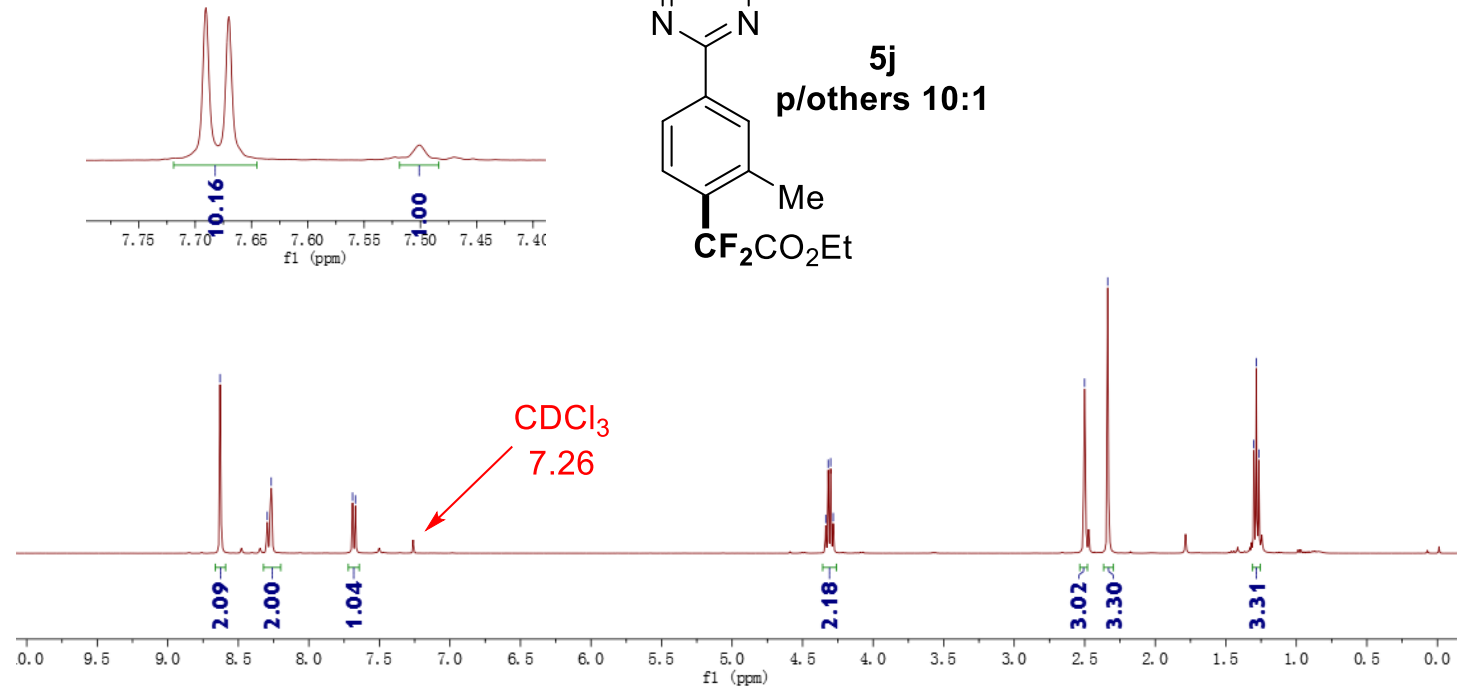

${ }^{19}$ F NMR (376 MHz, CDCl $)$ of $5 j$

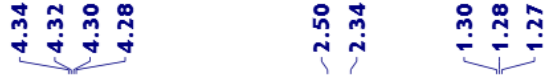

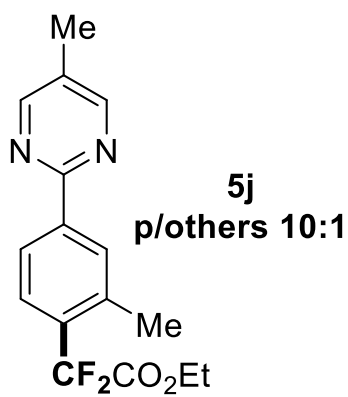


${ }^{13} \mathrm{C}$ NMR (100 MHz, $\left.\mathrm{CDCl}_{3}\right)$ of $5 \mathbf{j}$
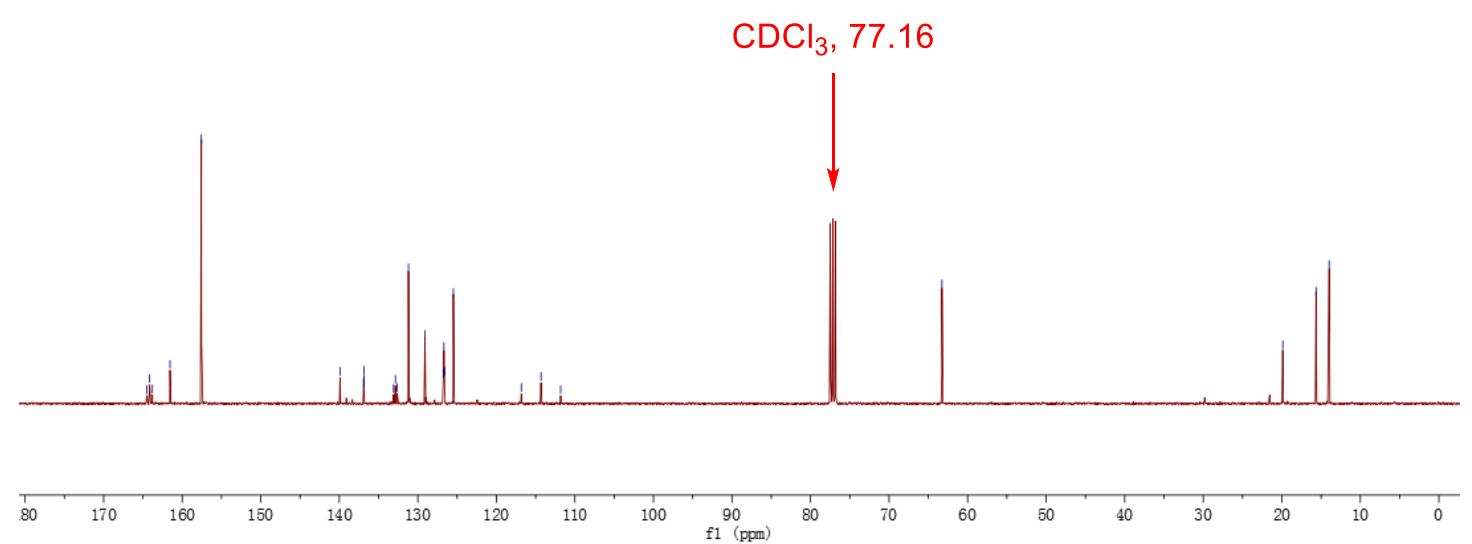

${ }^{1} \mathrm{H}$ NMR (400 MHz, $\left.\mathrm{CDCl}_{3}\right)$ of $5 \mathrm{k}$

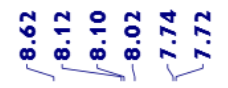

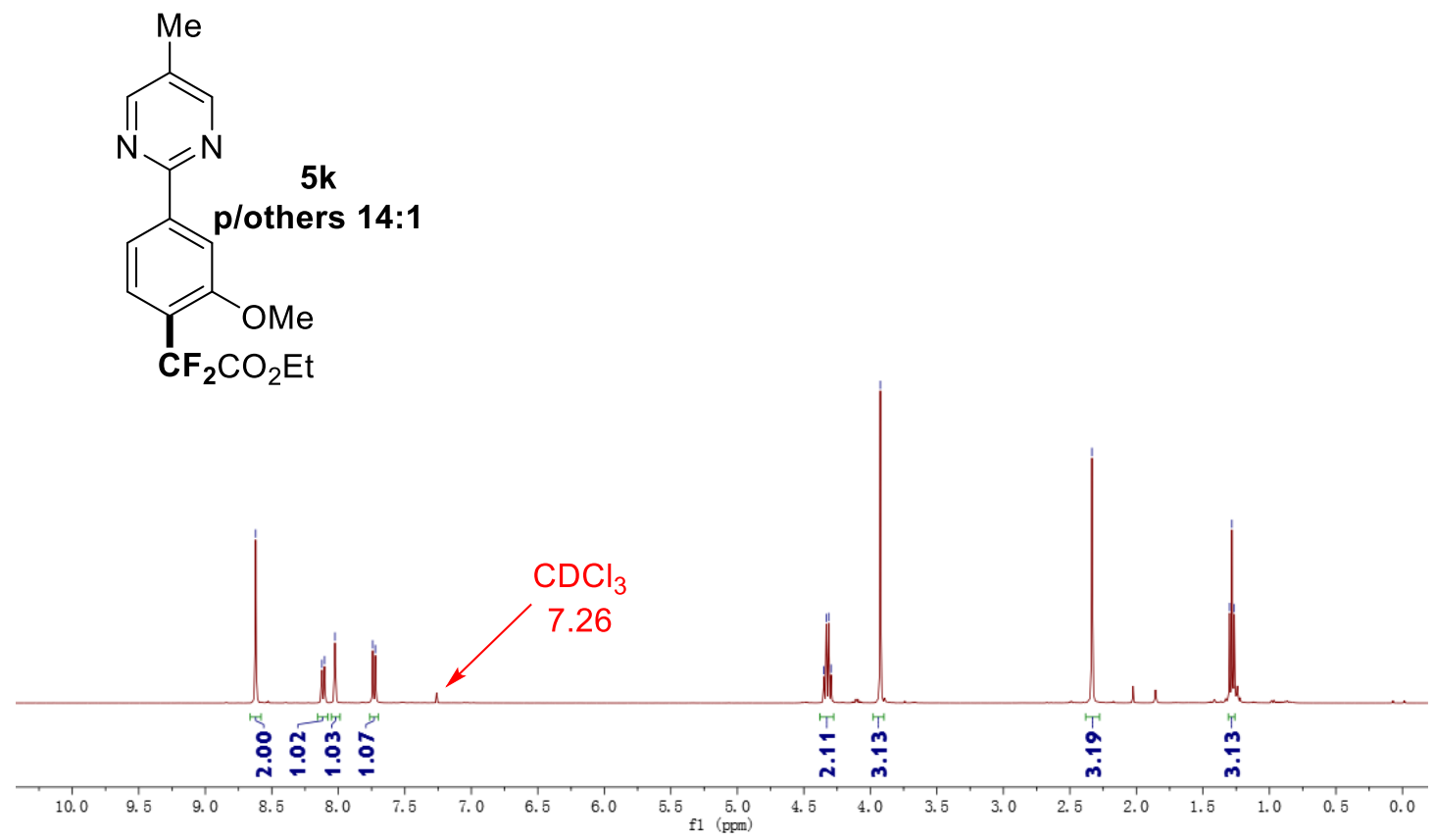




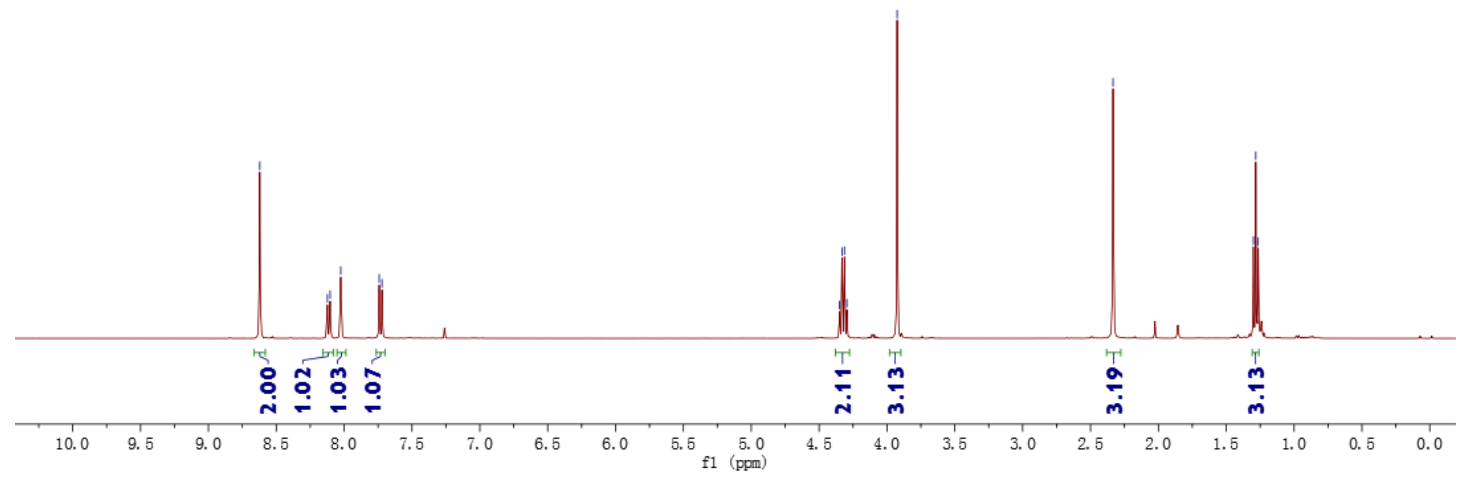

${ }^{19} \mathrm{~F}$ NMR (376 MHz, $\left.\mathrm{CDCl}_{3}\right)$ of $5 \mathrm{k}$

$$
\stackrel{\infty}{\stackrel{\infty}{0}}
$$

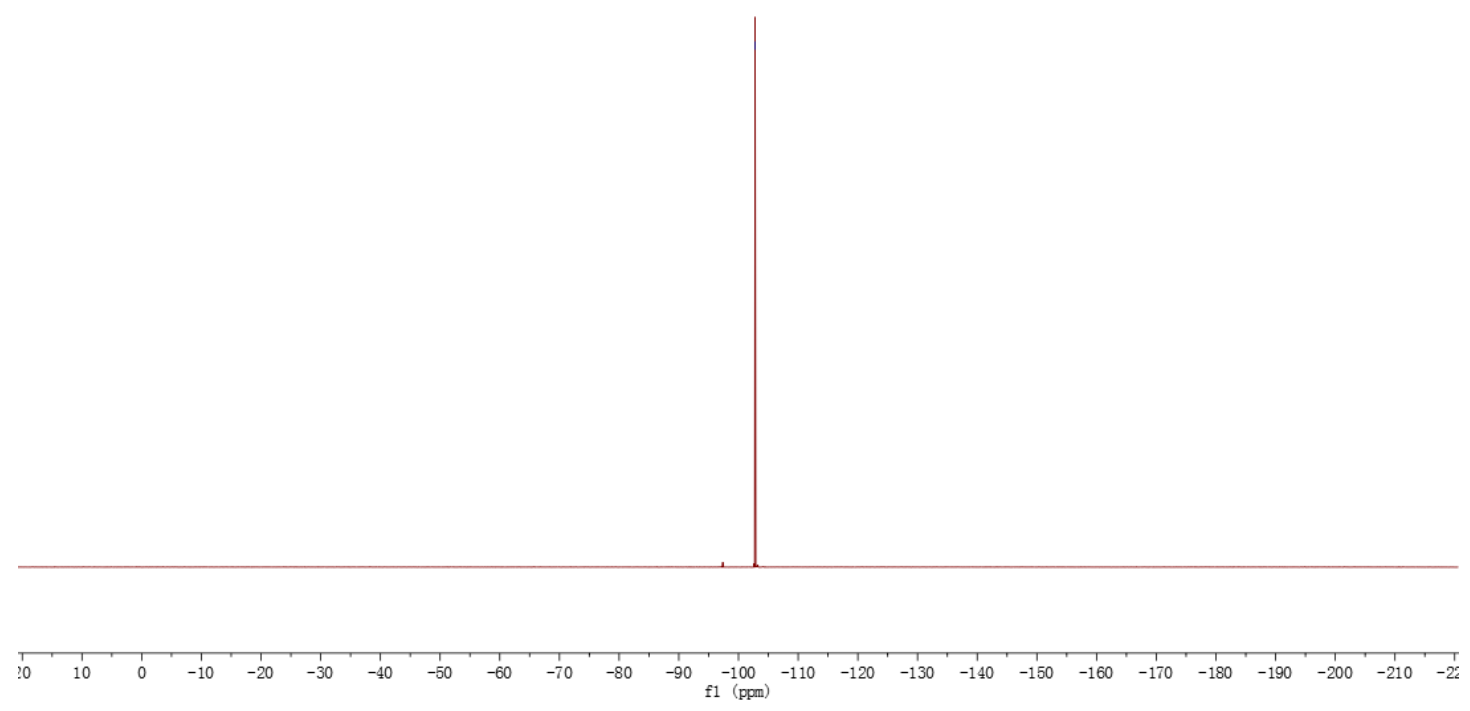


${ }^{13} \mathrm{C}$ NMR (100 MHz, $\left.\mathrm{CDCl}_{3}\right)$ of $5 \mathrm{k}$

๓

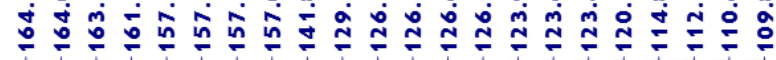

$\stackrel{0}{2}$

กै

in
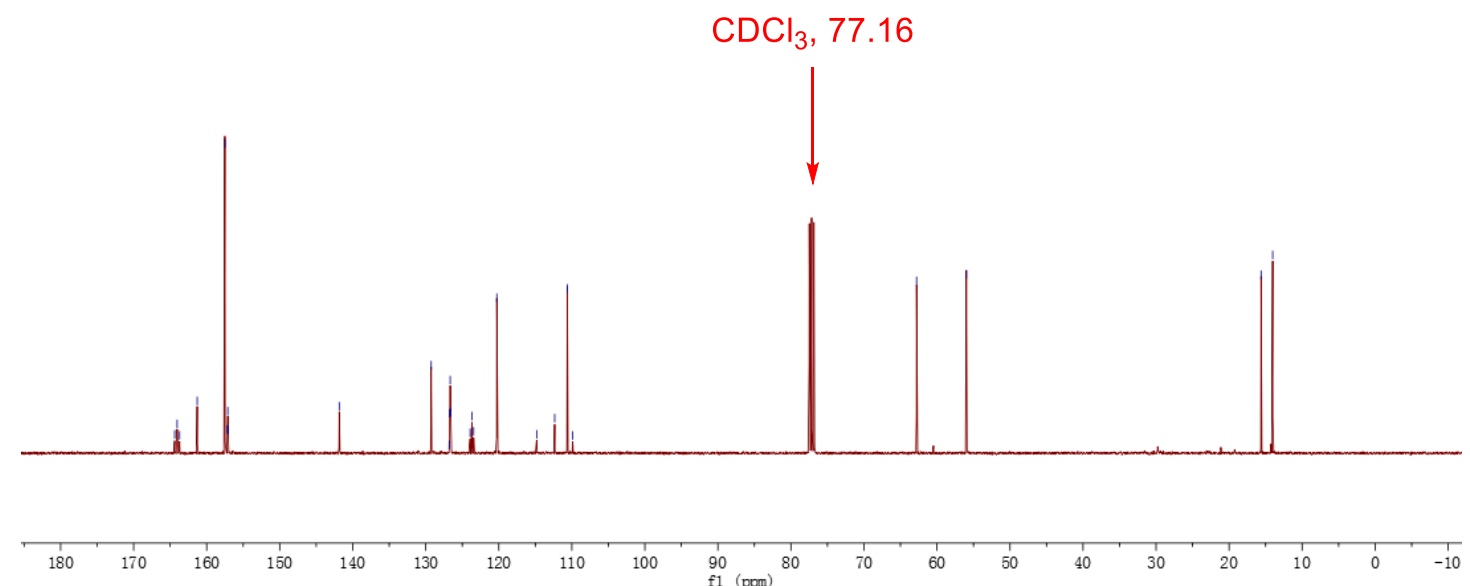

${ }^{1} \mathrm{H}$ NMR (400 $\left.\mathrm{MHz}, \mathrm{CDCl}_{3}\right)$ of $5 \mathrm{I}$

公岱

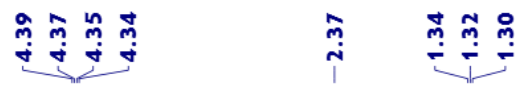<smiles>CCOC(=O)c1ccc(-c2ncc(C)cn2)cc1F</smiles>

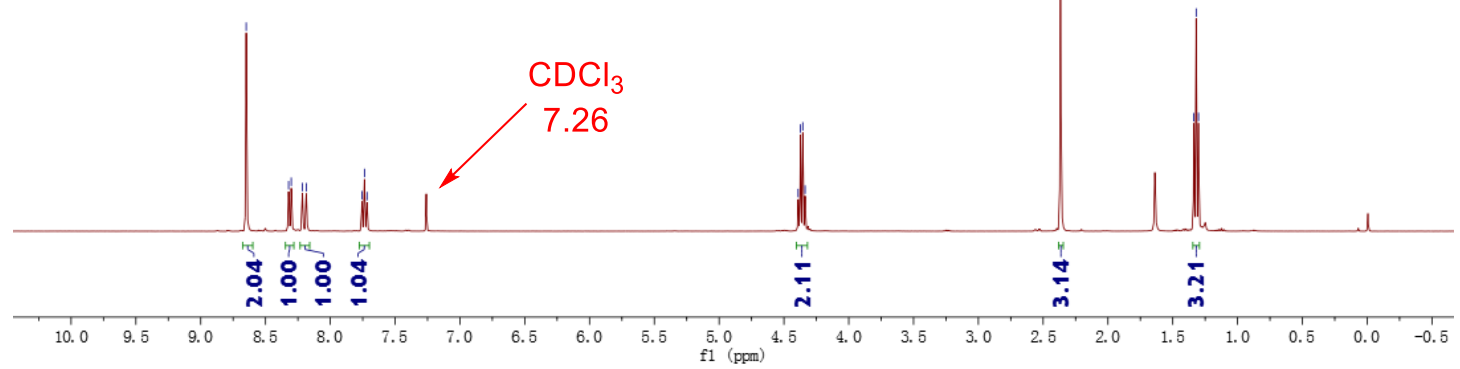


${ }^{19} \mathrm{~F}$ NMR $\left(376 \mathrm{MHz}, \mathrm{CDCl}_{3}\right)$ of 51
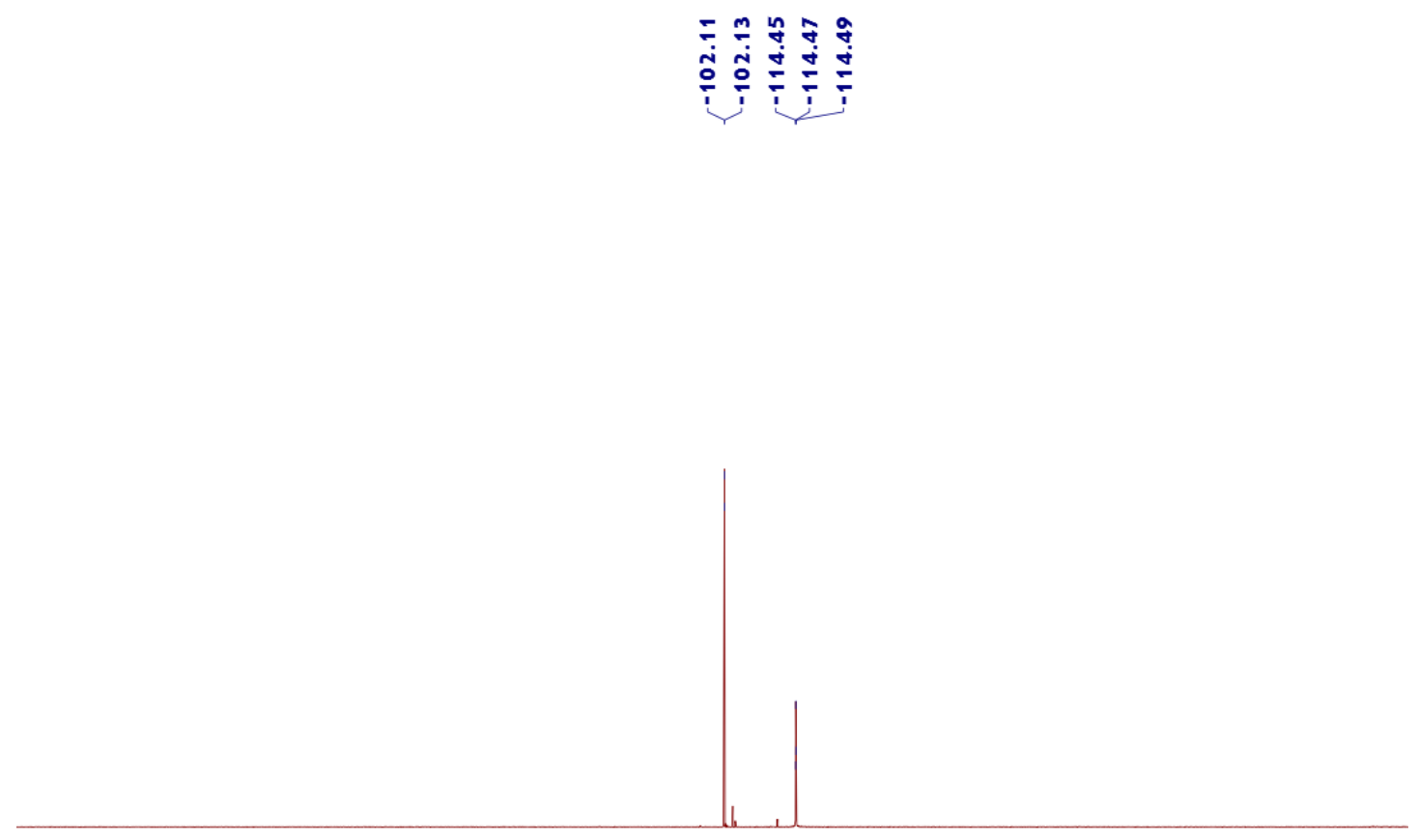

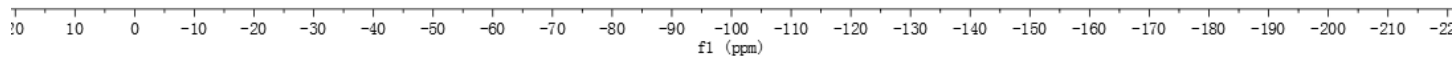

${ }^{13} \mathrm{C}$ NMR (100 MHz, $\left.\mathrm{CDCl}_{3}\right)$ of $5 \mathrm{l}$

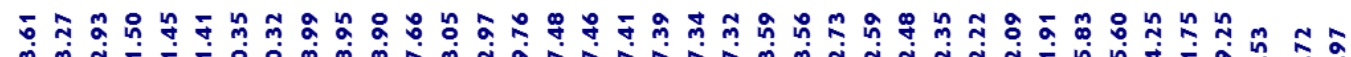

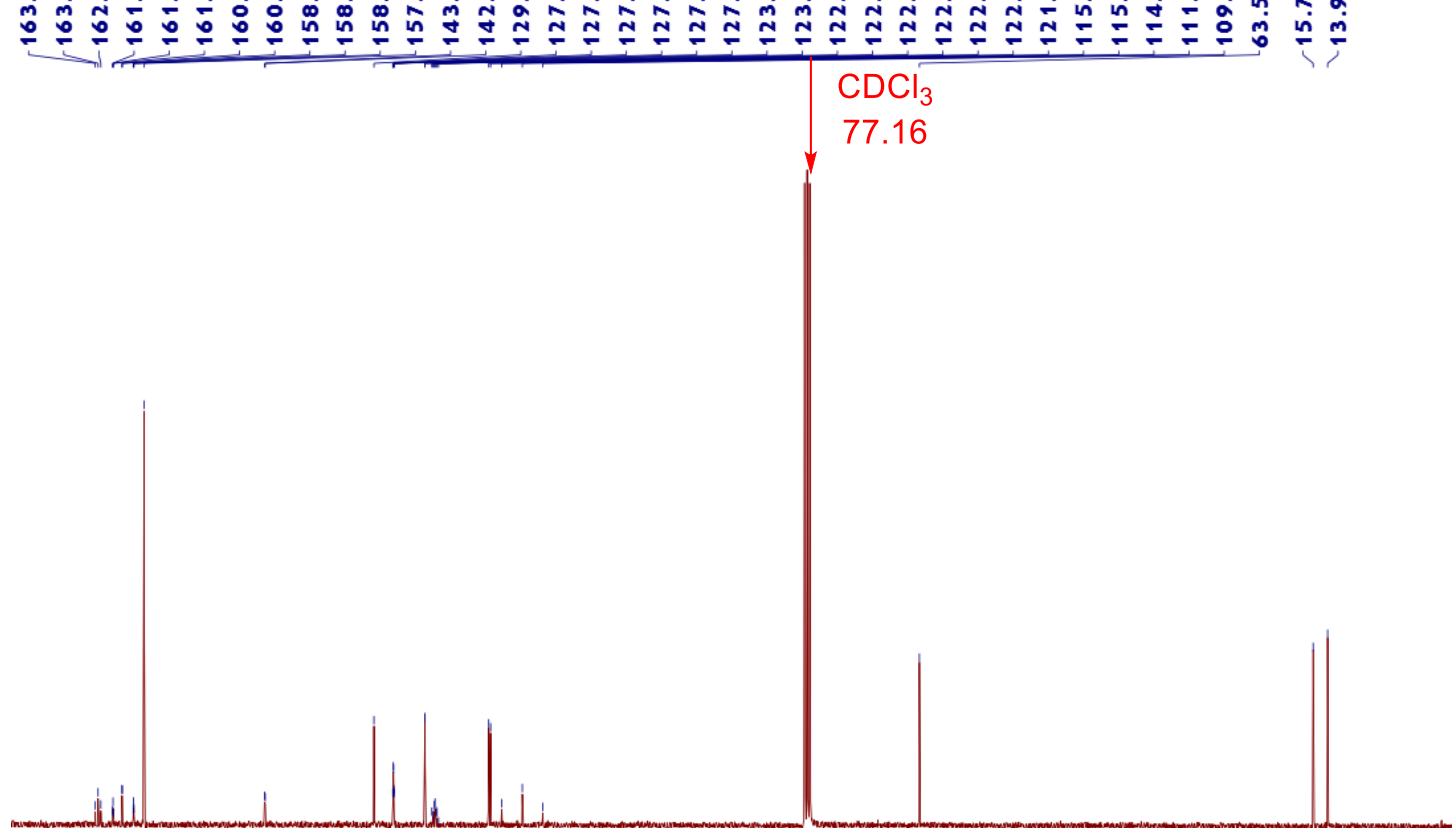

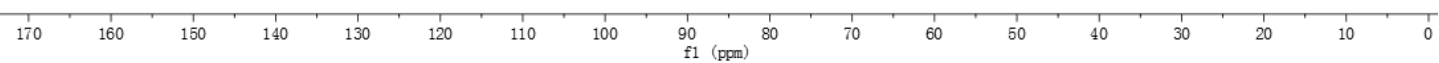


${ }^{1} \mathrm{H}$ NMR (400 MHz, $\left.\mathrm{CDCl}_{3}\right)$ of $5 \mathrm{~m}$

点

莳 导

$\stackrel{m}{i} \quad \stackrel{m}{\stackrel{m}{m} \stackrel{m}{m}}$<smiles>CCOC(=O)c1ccc(-c2ncc(C)cn2)cc1C#N</smiles>

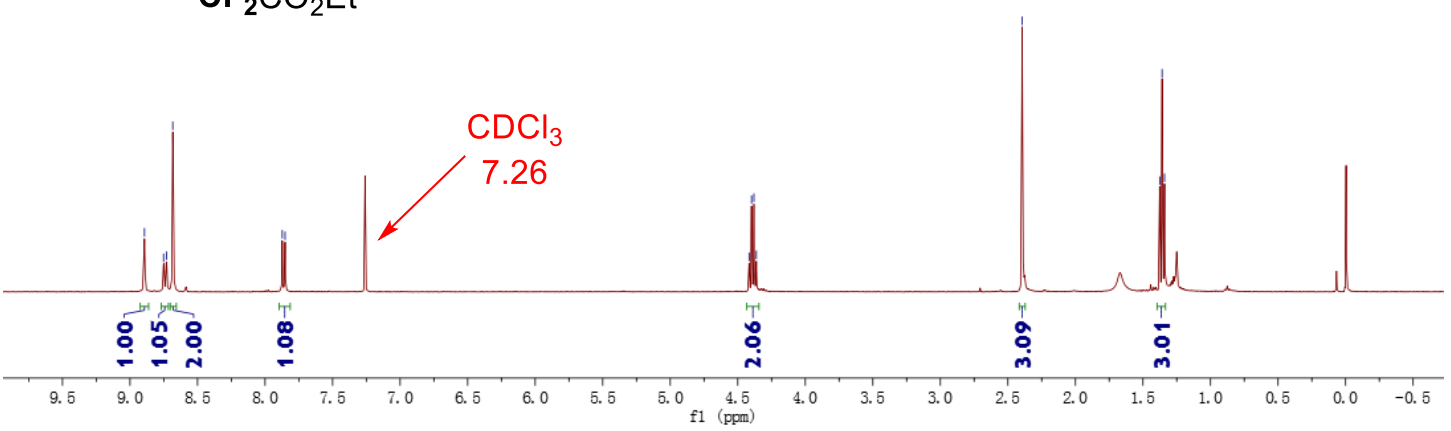

${ }^{19}$ F NMR (376 MHz, CDCl $)$ of $5 \mathrm{~m}$

$\frac{5}{1}$

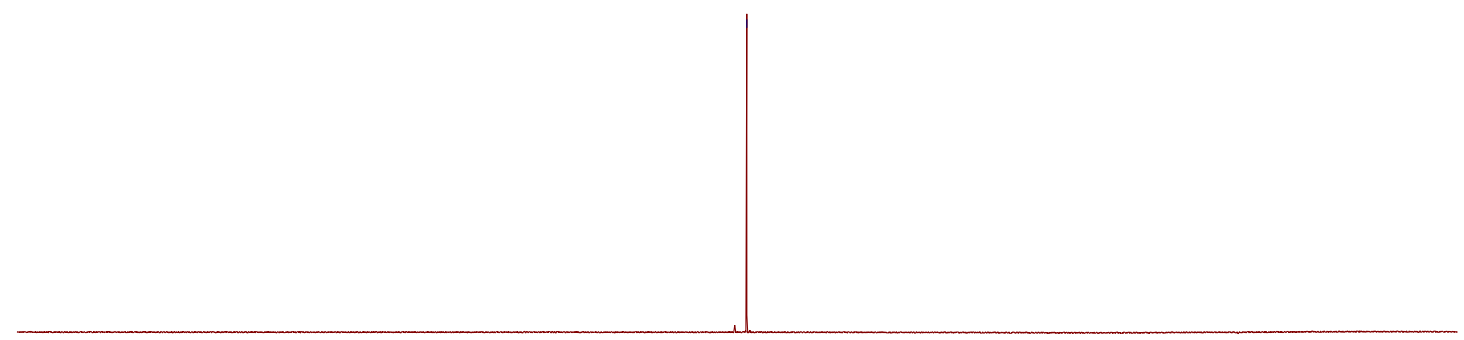


${ }^{13} \mathrm{C}$ NMR (100 MHz, $\left.\mathrm{CDCl}_{3}\right)$ of $5 \mathrm{~m}$

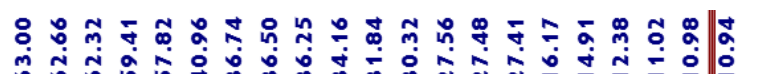

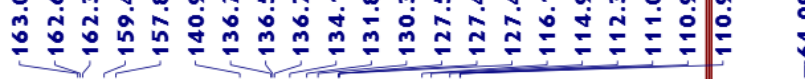

$\therefore a$

$\stackrel{\text { in }}{=}$

$\mathrm{CDCl}_{3}, 77.16$

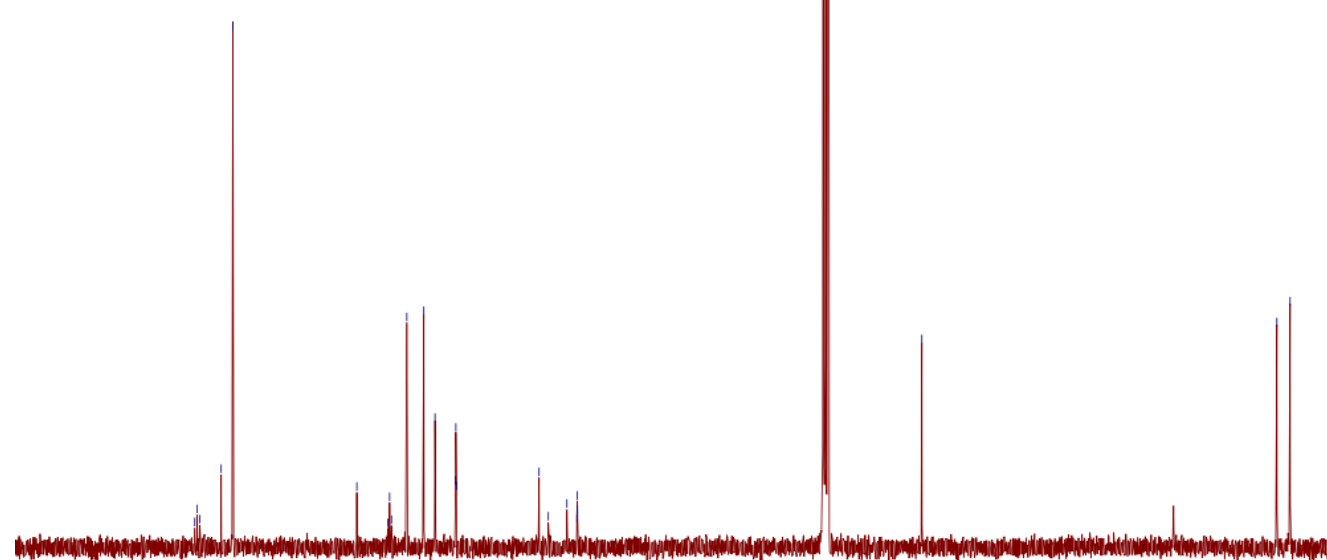

$\begin{array}{llllllll}180 & 170 & 160 & 150 & 140 & 130 & 120 & 110\end{array}$

${ }^{1} \mathrm{H}$ NMR (400 MHz, CDCl3) of $5 n$

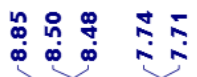

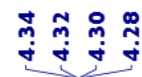

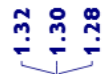
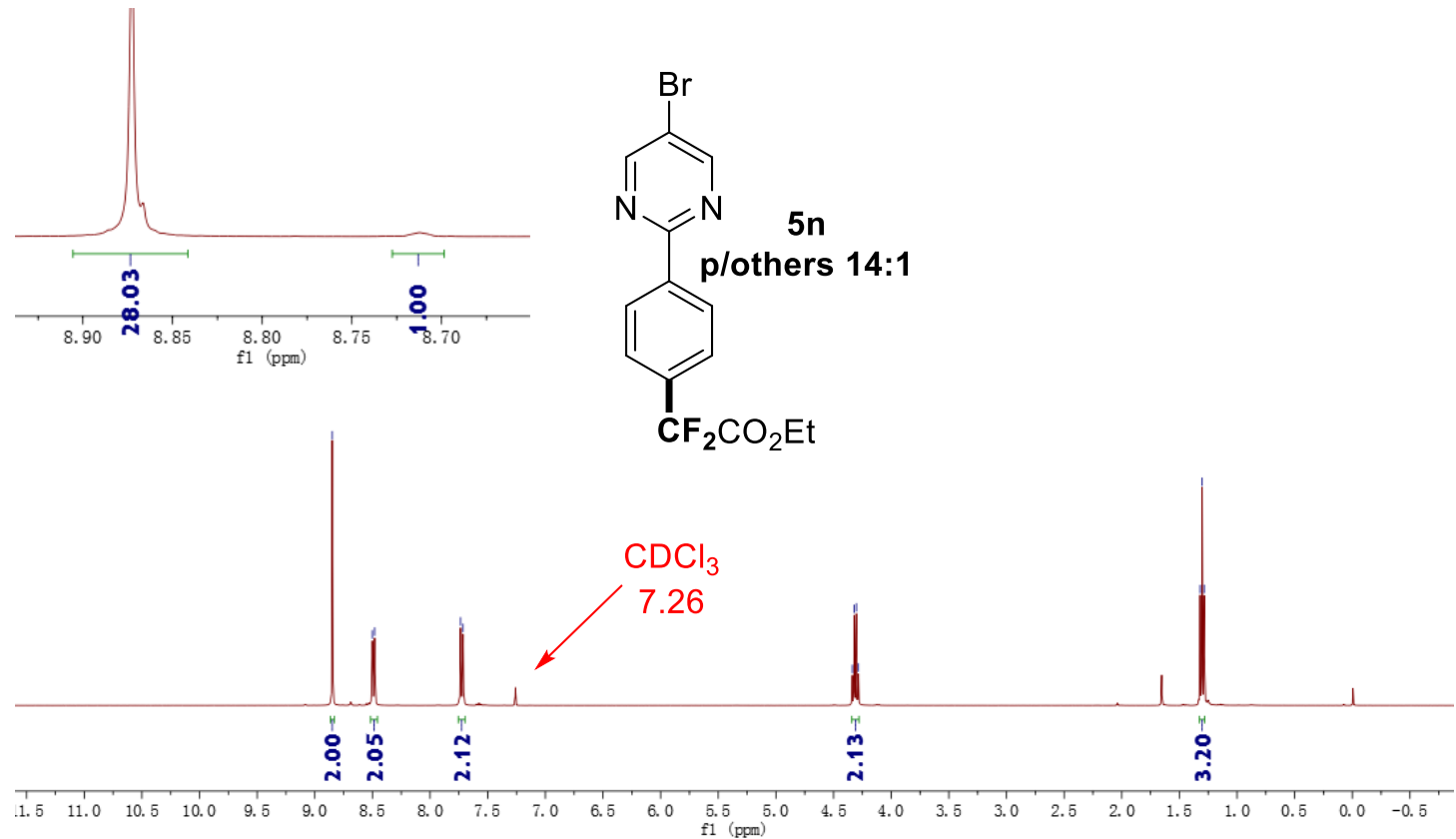

${ }^{19} \mathrm{~F}$ NMR (376 $\left.\mathrm{MHz}, \mathrm{CDCl}_{3}\right)$ of $5 \mathrm{n}$ 


\section{$\stackrel{\substack{* \\ \dot{0}}}{i}$}

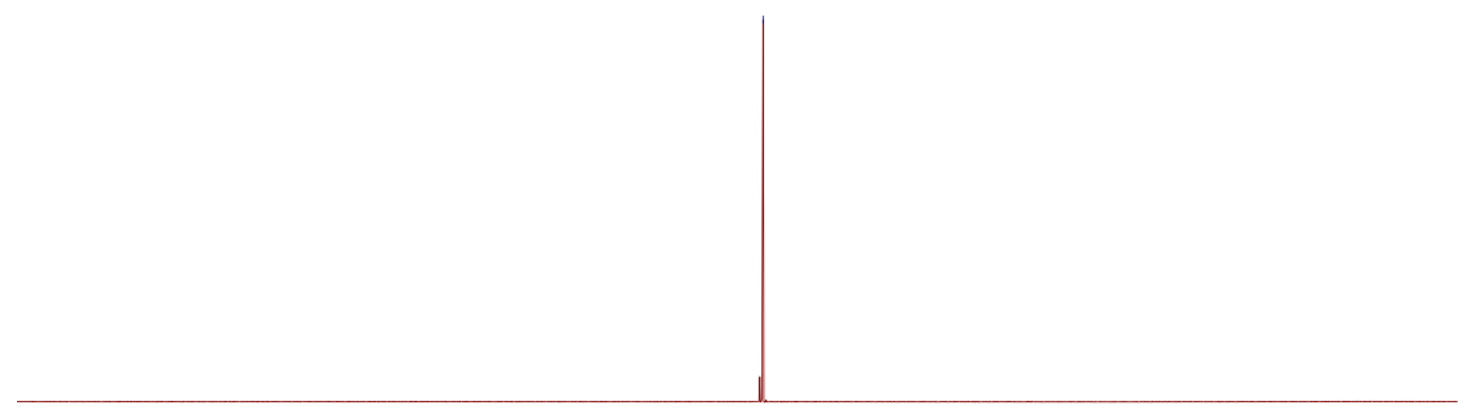

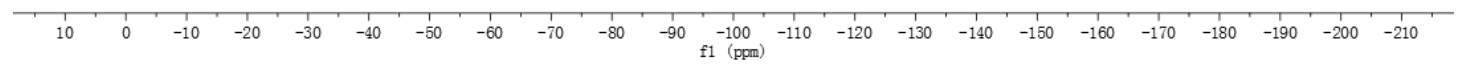

${ }^{13} \mathrm{C}$ NMR (100 MHz, $\left.\mathrm{CDCl}_{3}\right)$ of $5 \mathrm{n}$

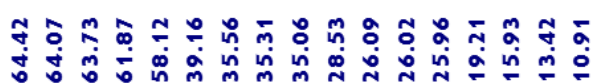

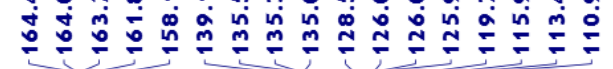

$\underset{\substack{\infty \\ i}}{i}$

$\stackrel{\circ}{\dot{I}}$

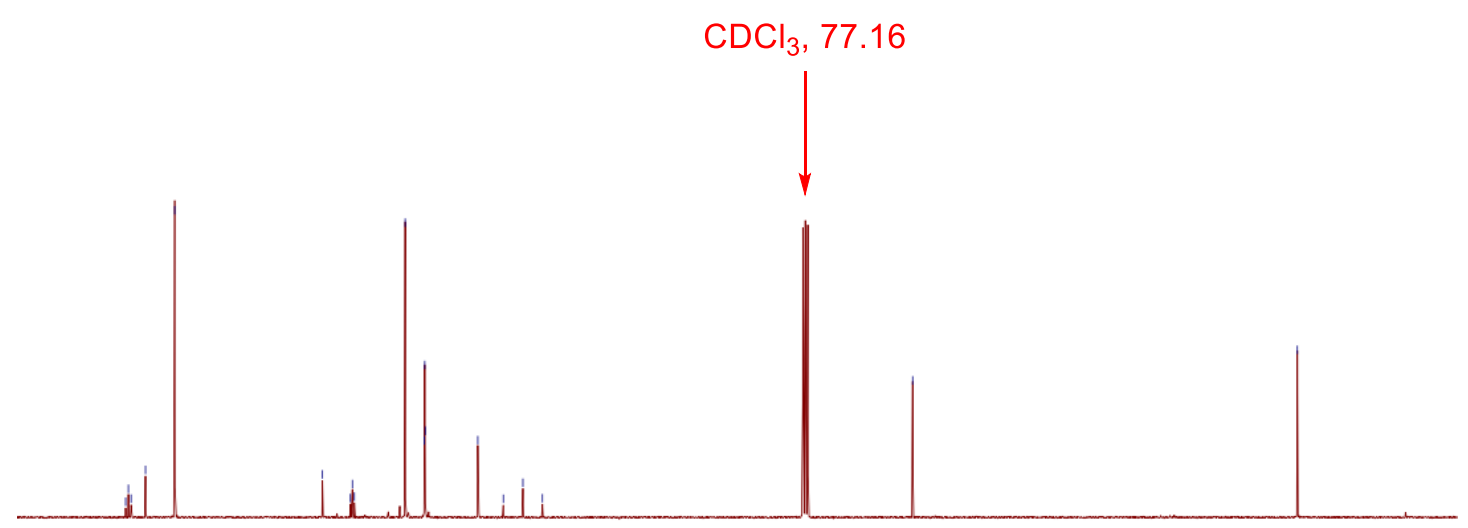

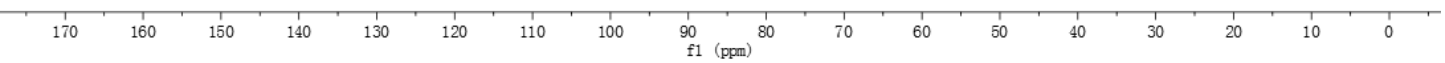


${ }^{1} \mathrm{H}$ NMR (400 MHz, $\left.\mathrm{CDCl}_{3}\right)$ of 50

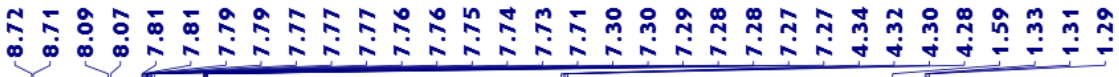
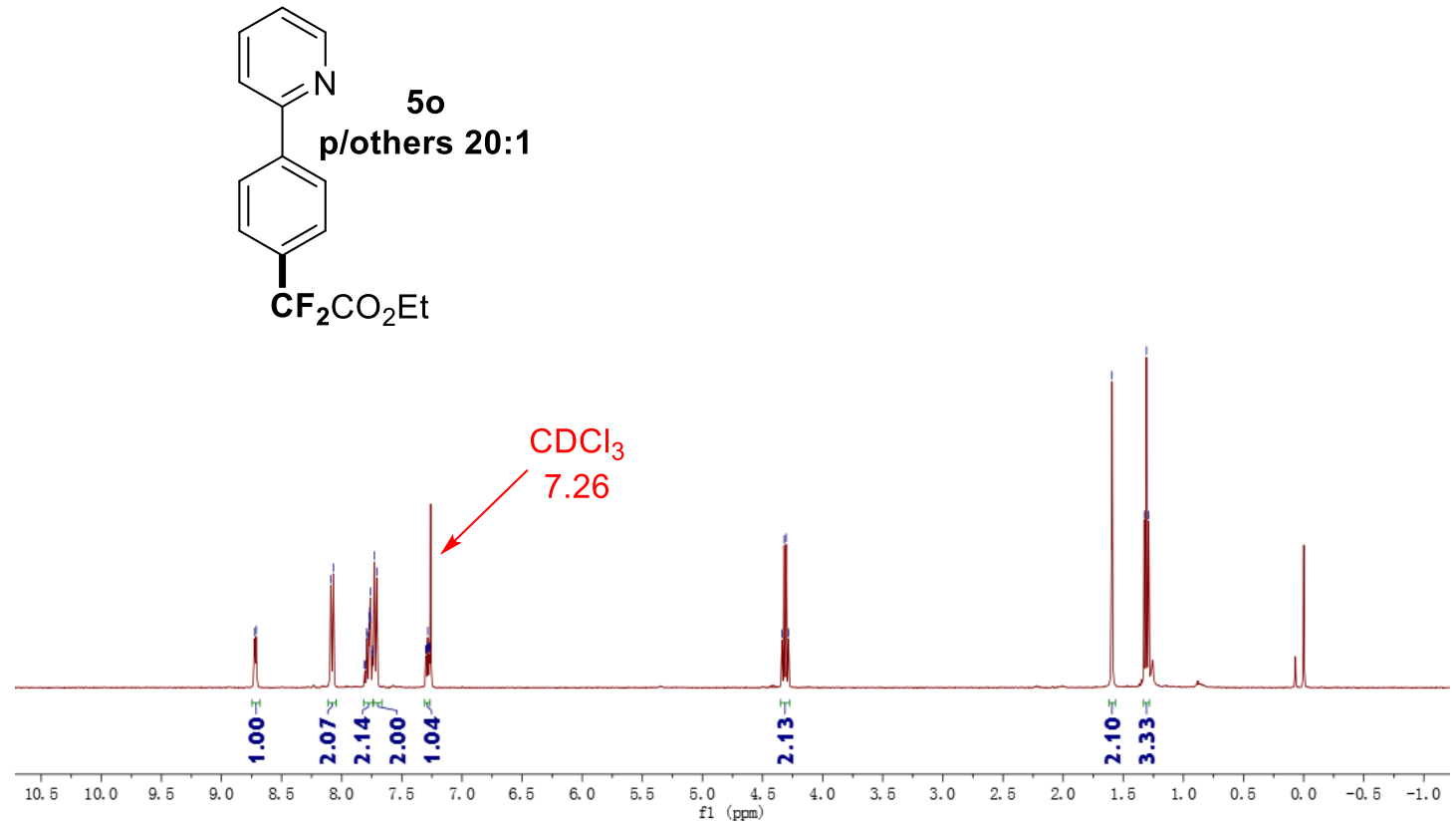

${ }^{19} \mathrm{~F}$ NMR (376 MHz, $\left.\mathrm{CDCl}_{3}\right)$ of 50 
${ }^{13} \mathrm{C}$ NMR (100 MHz, $\left.\mathrm{CDCl}_{3}\right)$ of 50

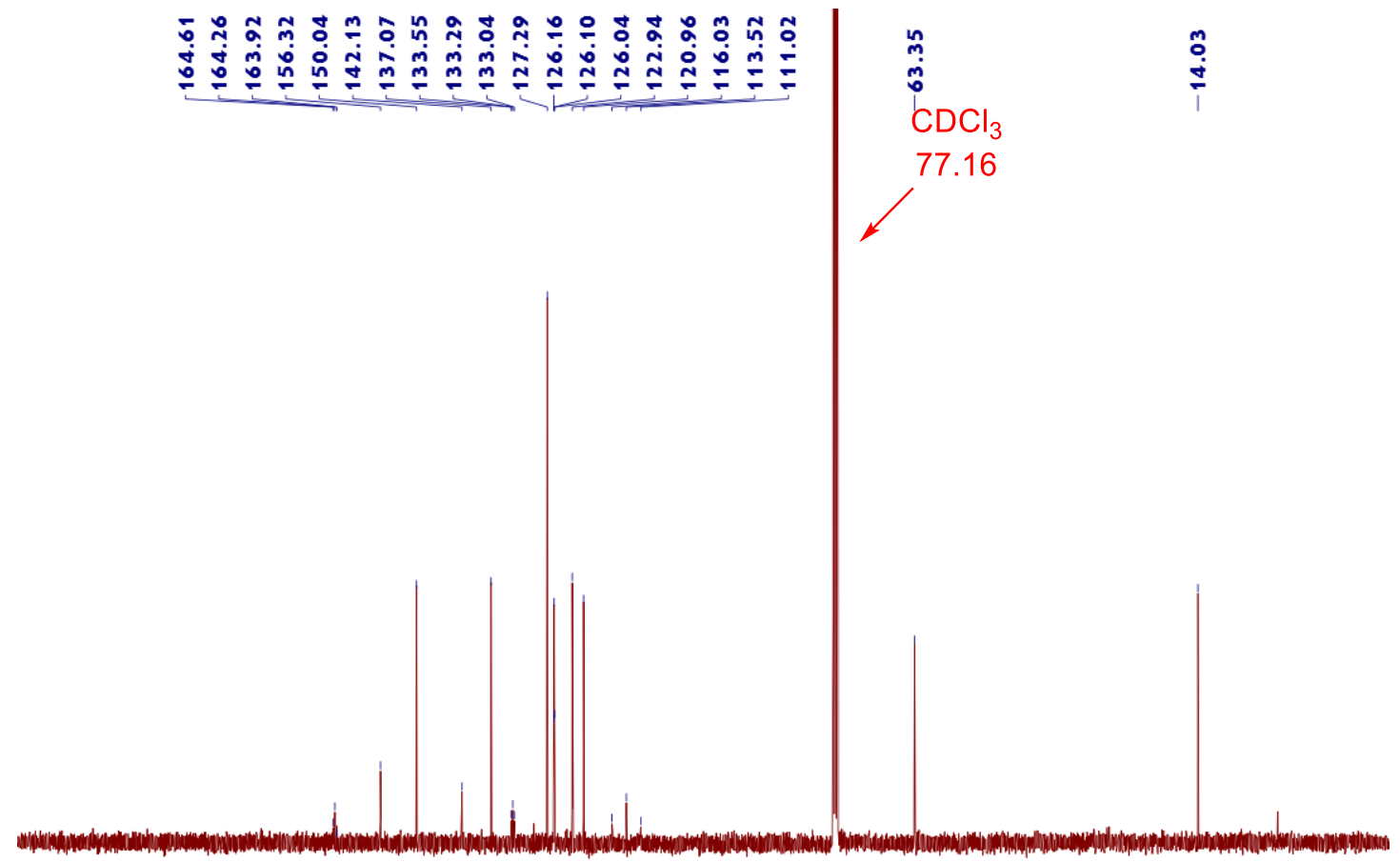

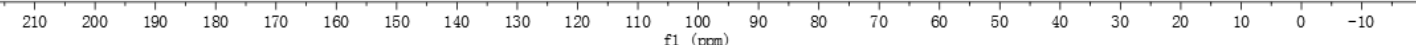

${ }^{1} \mathrm{H}$ NMR (400 MHz, $\left.\mathrm{CDCl}_{3}\right)$ of $50^{\prime}$

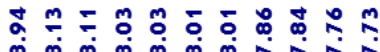

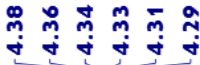

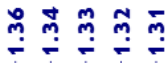

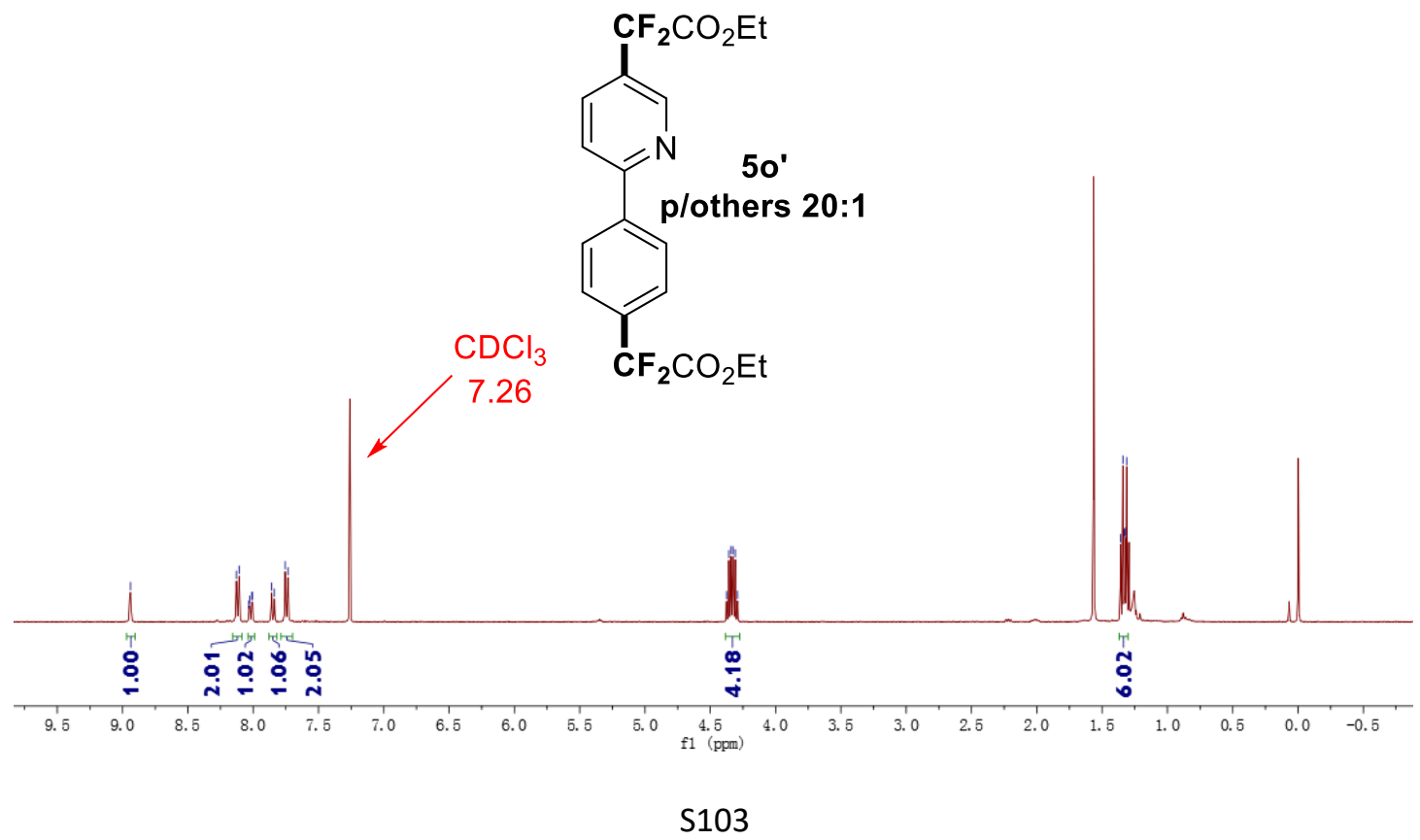


${ }^{19}$ F NMR (376 MHz, CDCl $)$ of $5 o^{\prime}$

$$
\stackrel{+}{\frac{m}{\dot{j}}} \stackrel{\stackrel{m}{\dot{0}}}{\frac{+}{i}}
$$

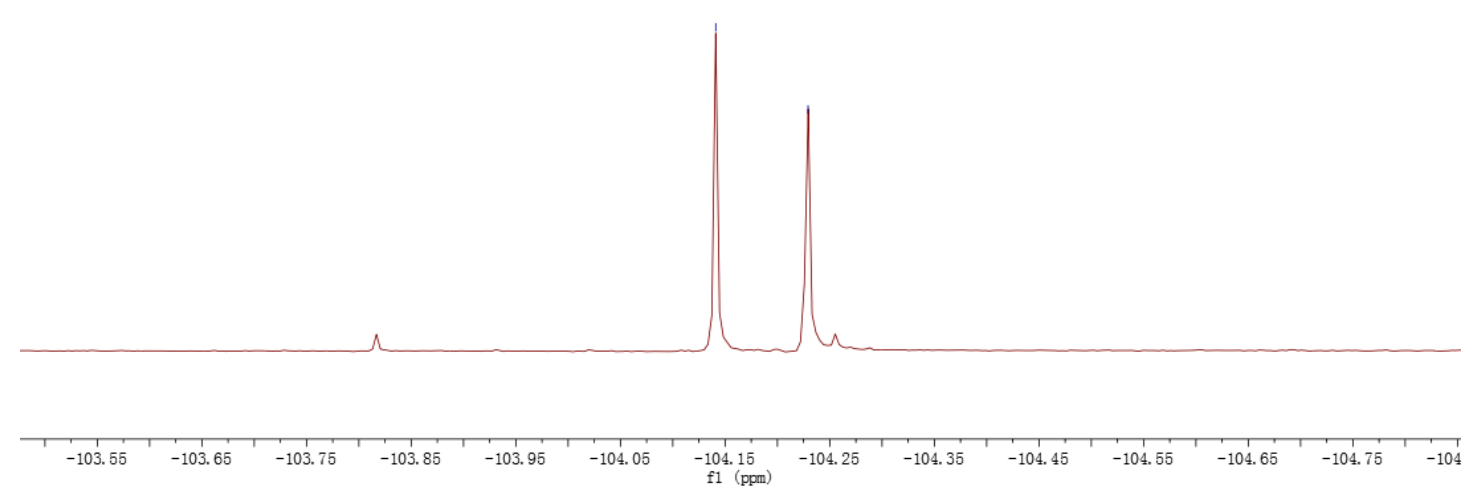

${ }^{1} \mathrm{H}$ NMR (400 MHz, $\left.\mathrm{CDCl}_{3}\right)$ of 7a

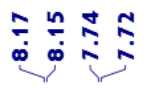

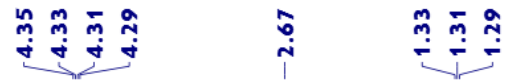

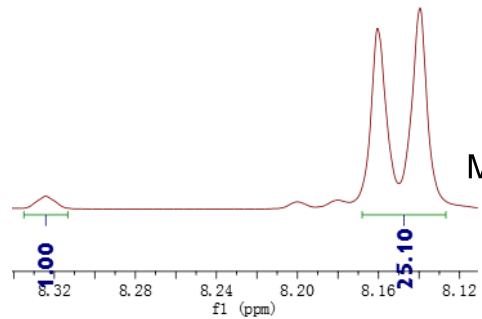<smiles></smiles>

p/others 12:1<smiles>CCOC(=O)C(F)(F)c1cccc(CC)c1</smiles>

$\mathrm{CDCl}_{3}$

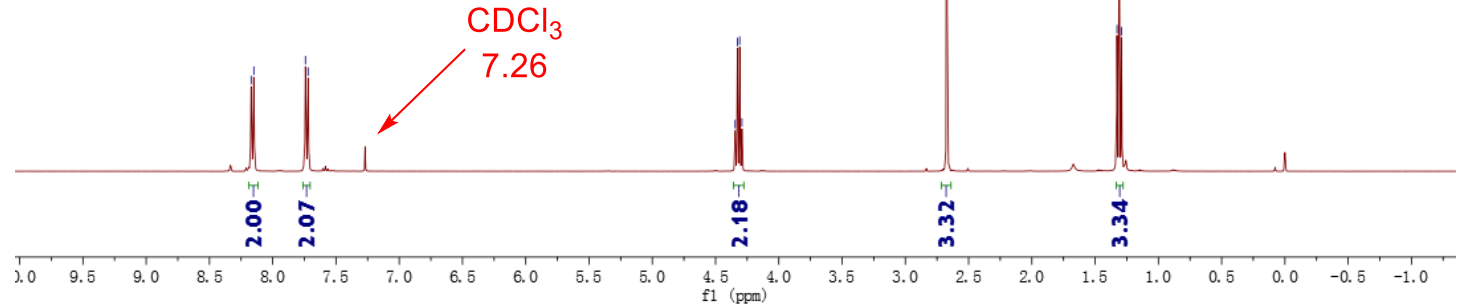


${ }^{19} \mathrm{~F}$ NMR $\left(376 \mathrm{MHz}, \mathrm{CDCl}_{3}\right)$ of $7 \mathrm{a}$

$$
\frac{\bar{m}}{\stackrel{m}{i}}
$$

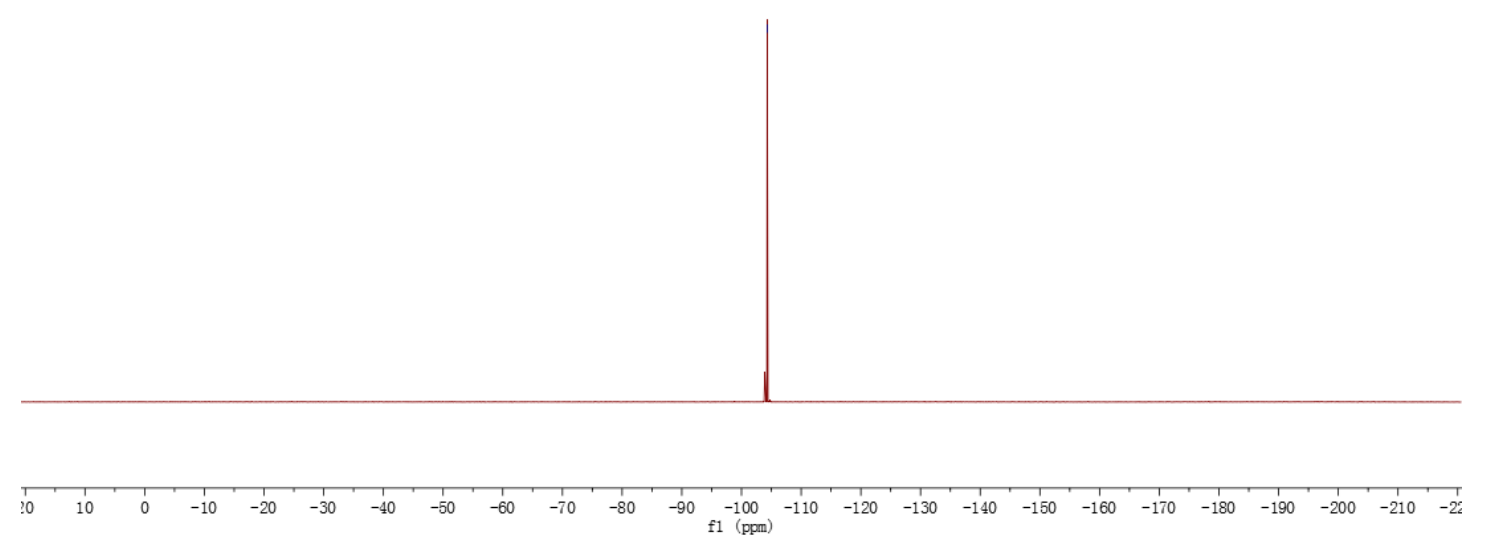

${ }^{13} \mathrm{C}$ NMR (100 MHz, $\left.\mathrm{CDCl}_{3}\right)$ of $7 \mathrm{a}$

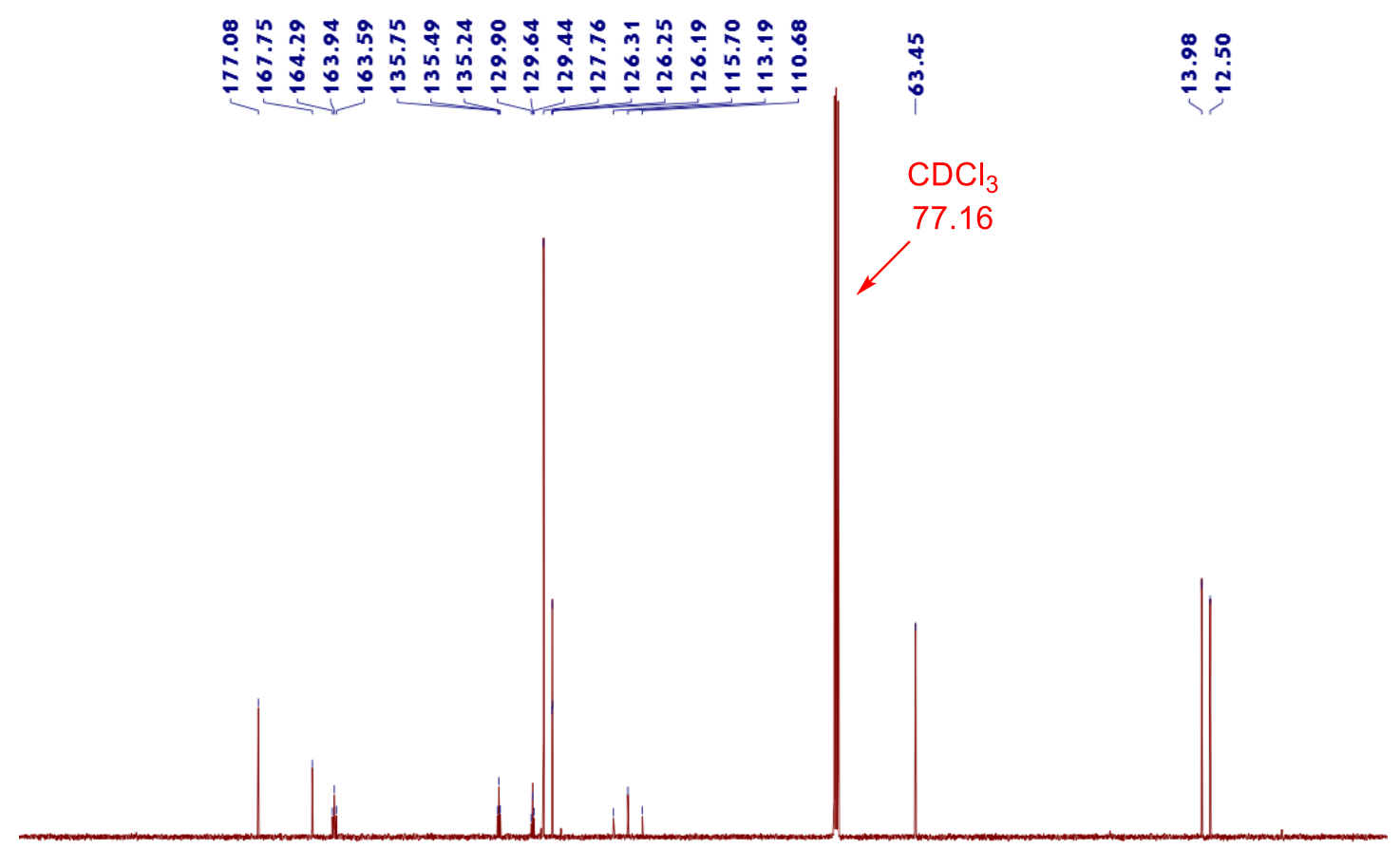

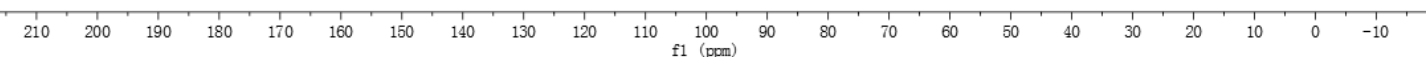


${ }^{1} \mathrm{H}$ NMR (400 MHz, CDCl$)$ of $7 \mathrm{~b}$

$$
\text { 禹華華 }
$$
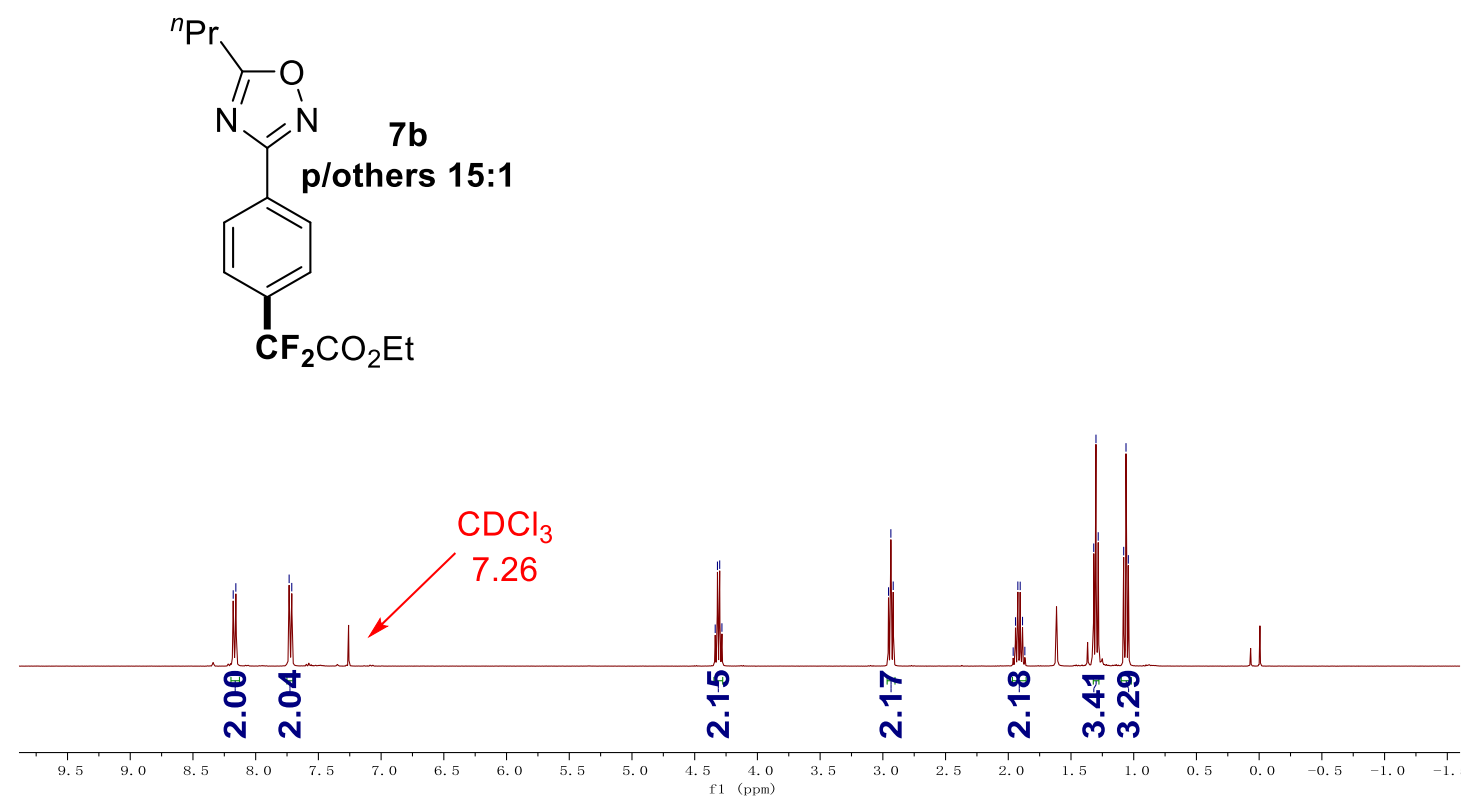

${ }^{19}$ F NMR $\left(376 \mathrm{MHz}, \mathrm{CDCl}_{3}\right)$ of $7 \mathrm{~b}$

$$
\text { ঙ̊ำ }
$$

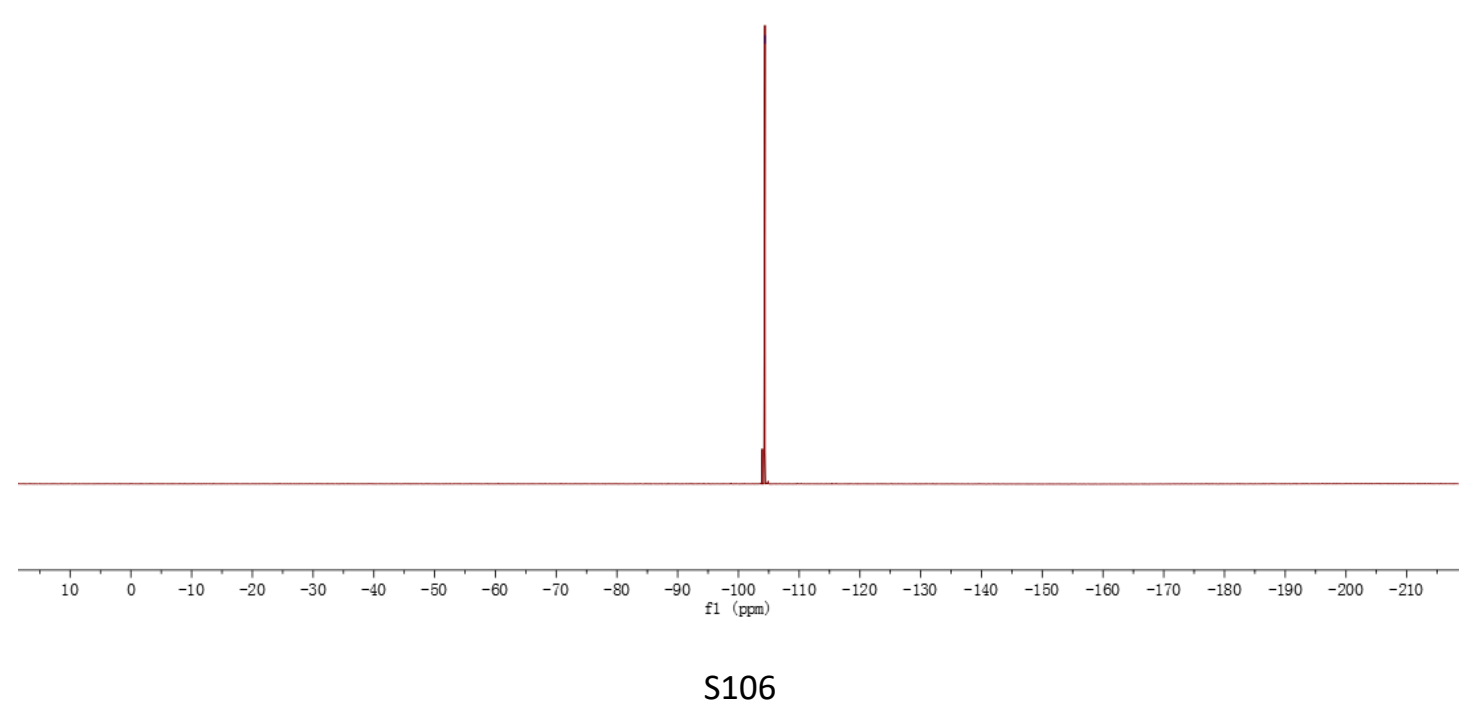


${ }^{13} \mathrm{C}$ NMR (100 $\left.\mathrm{MHz}, \mathrm{CDCl}_{3}\right)$ of $7 \mathrm{~b}$

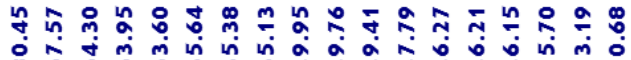

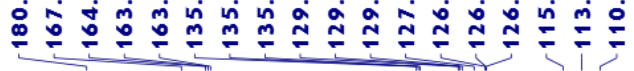

i̊n.

ㅇํำ

$\stackrel{\infty}{i} \stackrel{m}{\stackrel{m}{i}}$

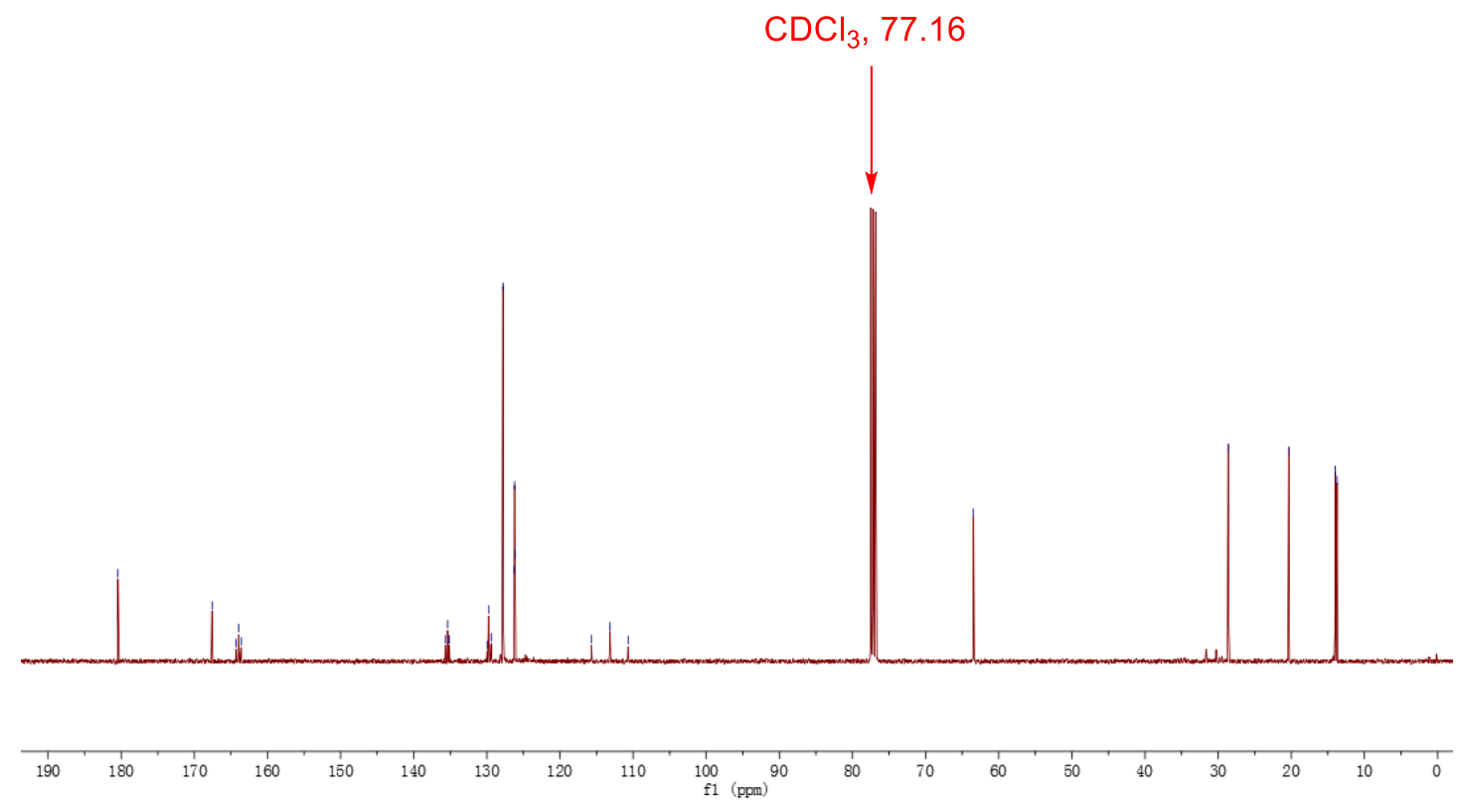

${ }^{1} \mathrm{H}$ NMR (400 MHz, $\left.\mathrm{CDCl}_{3}\right)$ of $7 \mathrm{c}$

$$
\text { Nị }
$$
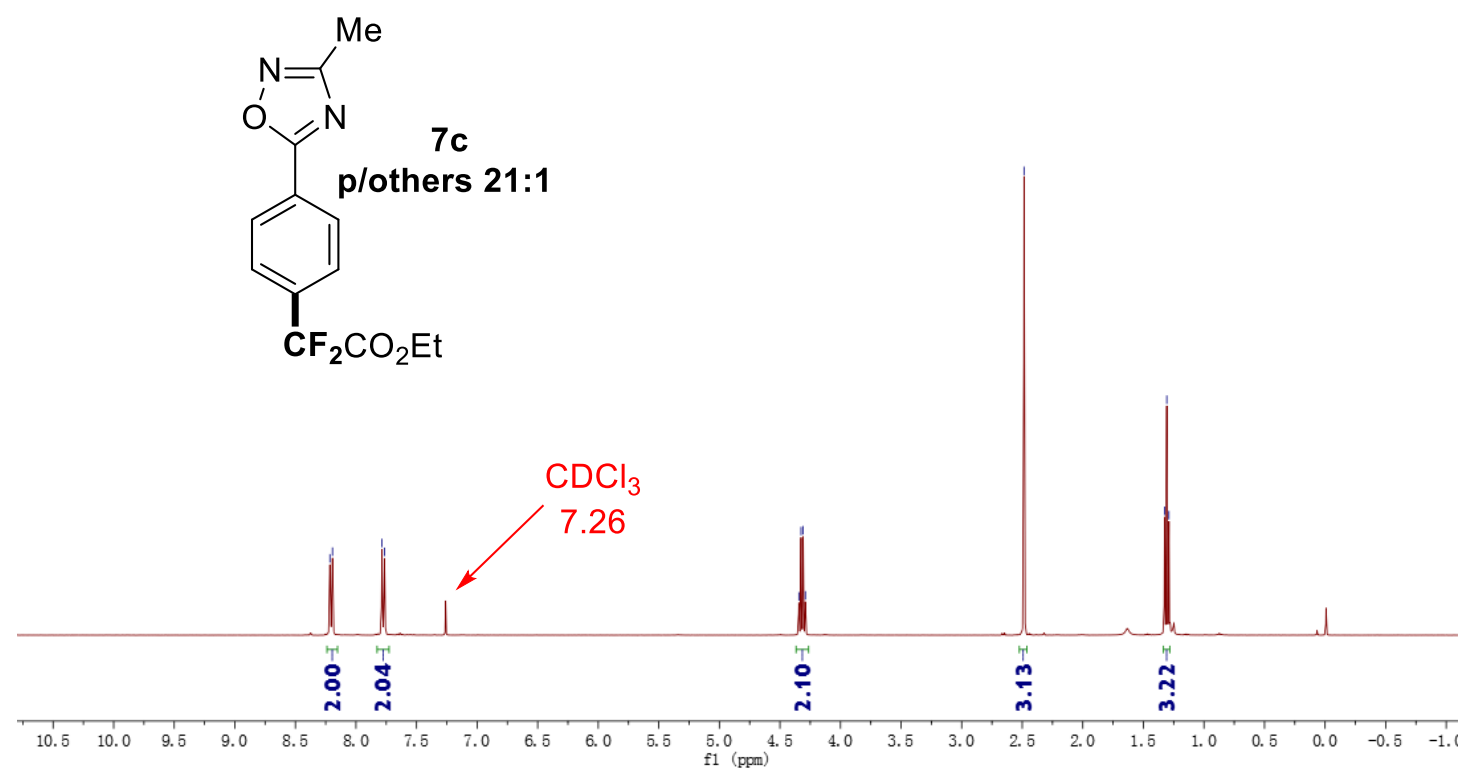
${ }^{19}$ F NMR (376 MHz, $\left.\mathrm{CDCl}_{3}\right)$ of $7 \mathrm{c}$

$$
\text { i̊ }
$$

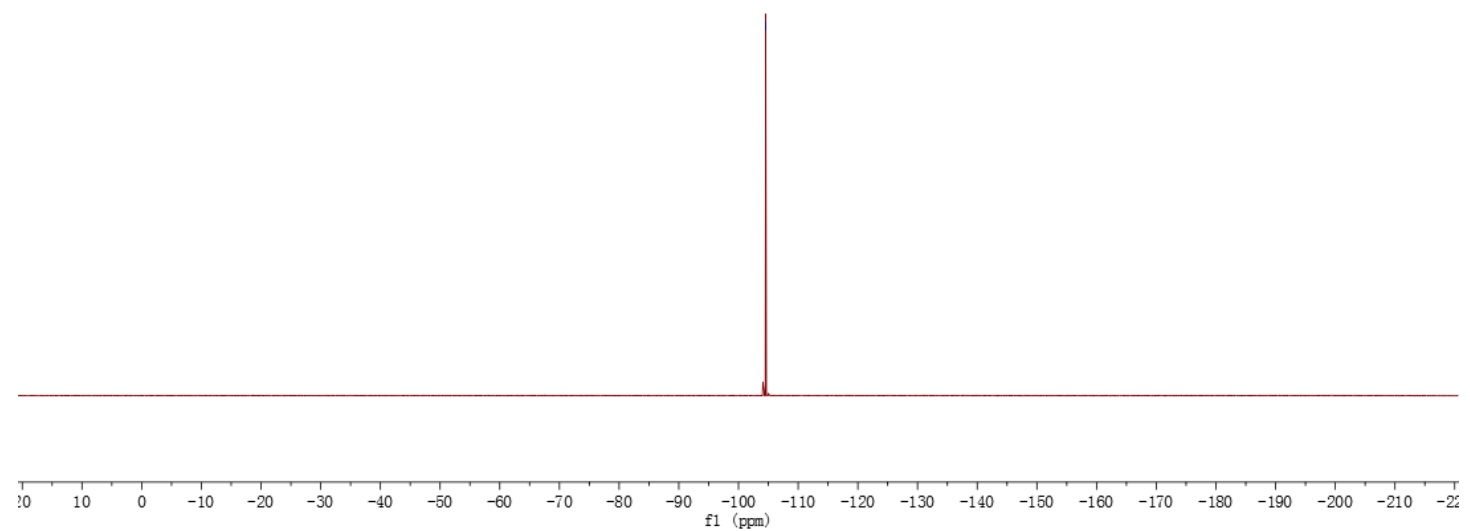

${ }^{13} \mathrm{C}$ NMR (100 $\left.\mathrm{MHz}, \mathrm{CDCl}_{3}\right)$ of 7c

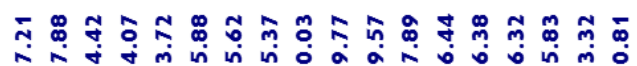
^

ณึ

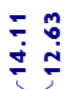

$\mathrm{CDCl}_{3}, 77.16$

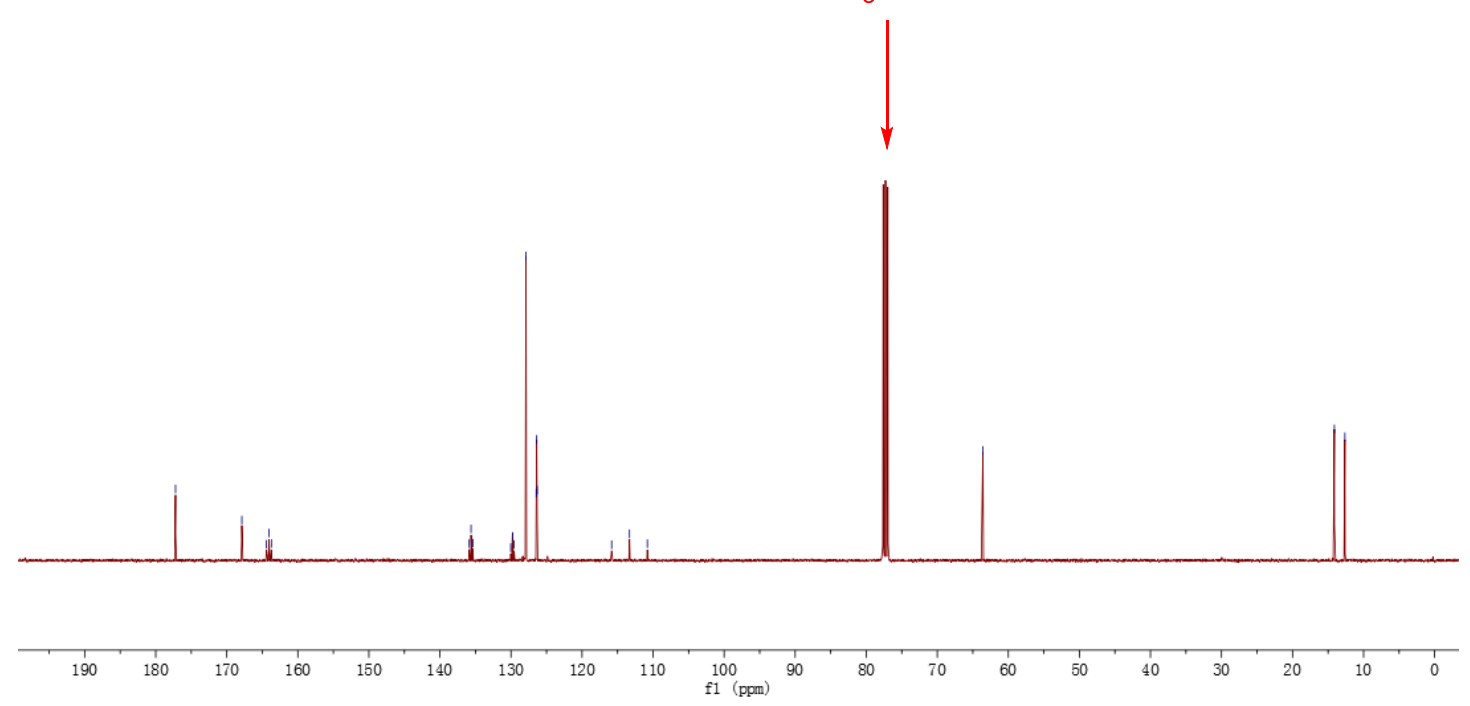


${ }^{1} \mathrm{H}$ NMR (400 MHz, CDCl$)$ of 7d

$\underset{\infty}{m}=\frac{m}{\infty}=\frac{0}{i}$

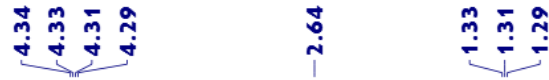<smiles>CCOC(=O)C(F)(F)c1ccc(-c2nnc(C)o2)cc1</smiles>

${ }^{19}$ F NMR (376 MHz, $\mathrm{CDCl}_{3}$ ) of $7 \mathrm{~d}$

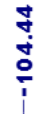

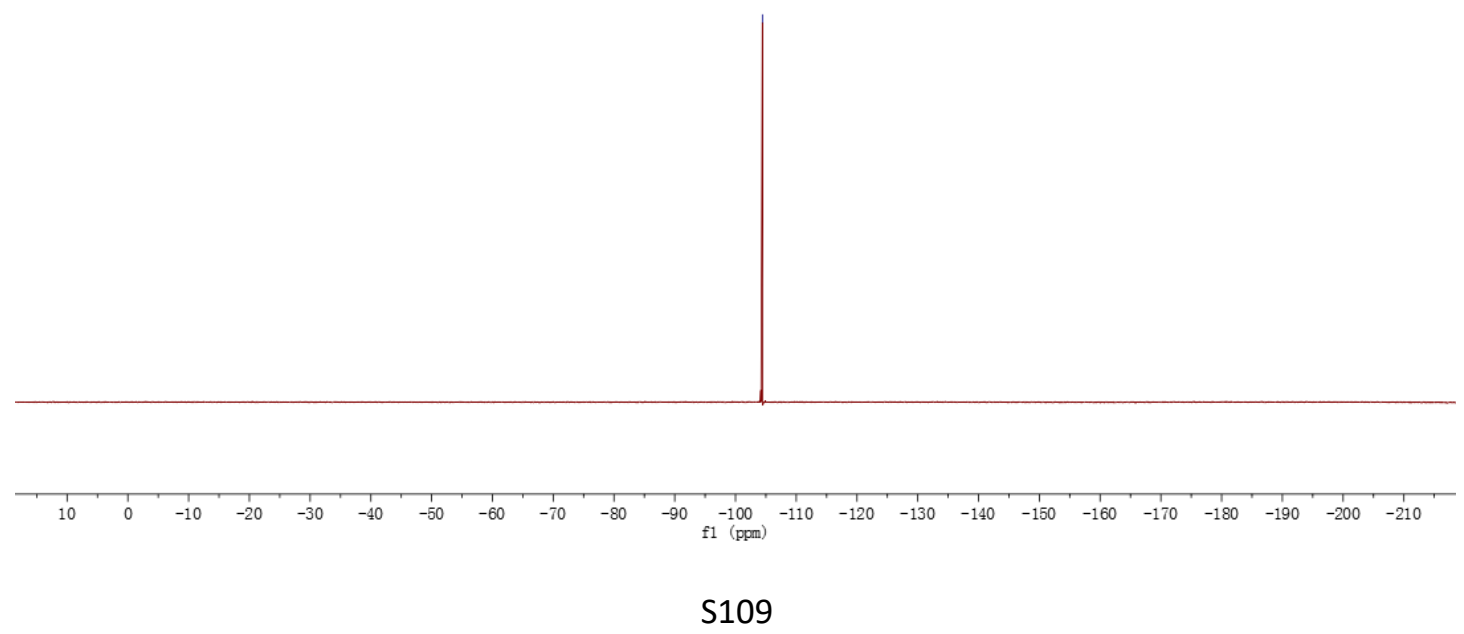


${ }^{13} \mathrm{C}$ NMR (100 $\left.\mathrm{MHz}, \mathrm{CDCl}_{3}\right)$ of 7d

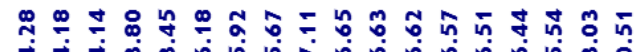

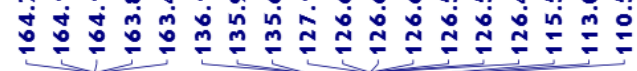

in

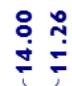

$\mathrm{CDCl}_{3}$

77.16

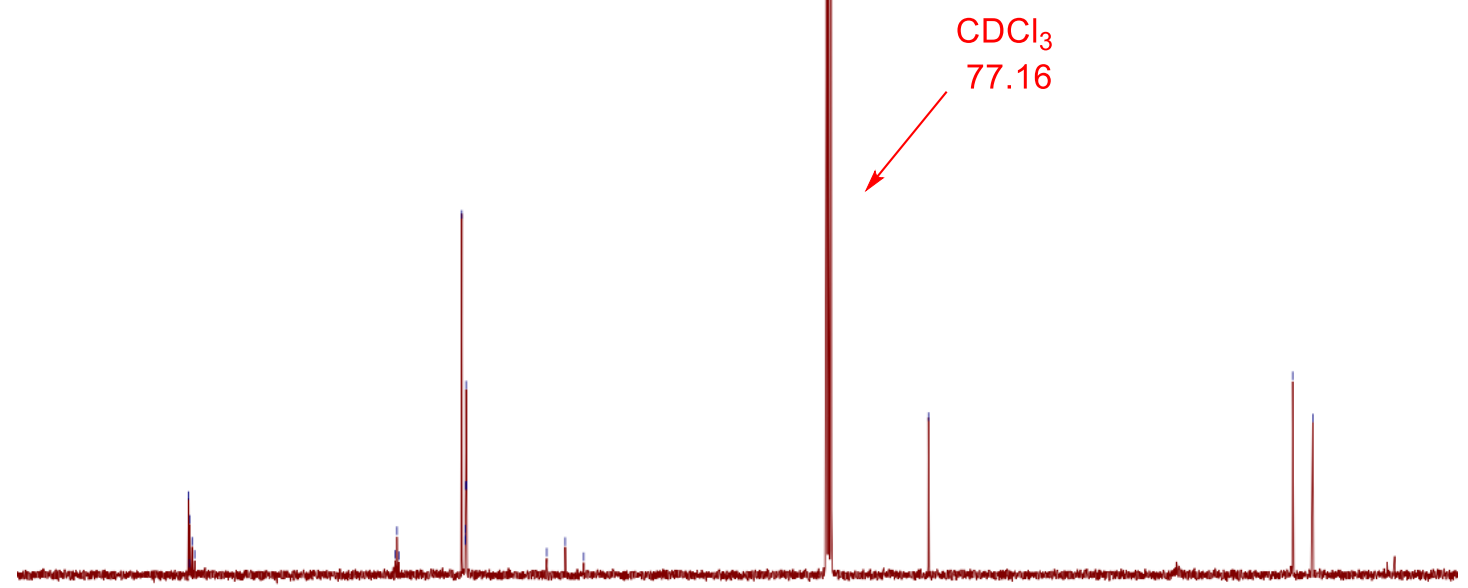

180

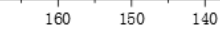

100

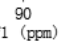

${ }^{1} \mathrm{H}$ NMR (400 MHz, $\left.\mathrm{CDCl}_{3}\right)$ of $7 \mathrm{e}$

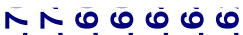

NNNNNN

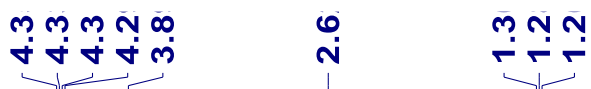

OMe

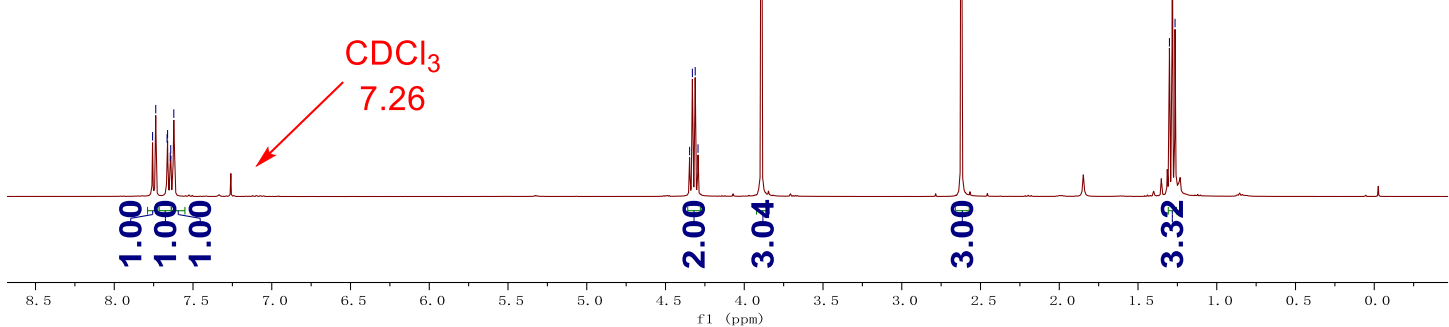


${ }^{19}$ F NMR (376 MHz, $\left.\mathrm{CDCl}_{3}\right)$ of $7 \mathrm{e}$

$$
\underset{\substack{\text { ị } \\ \stackrel{0}{i}}}{i}
$$

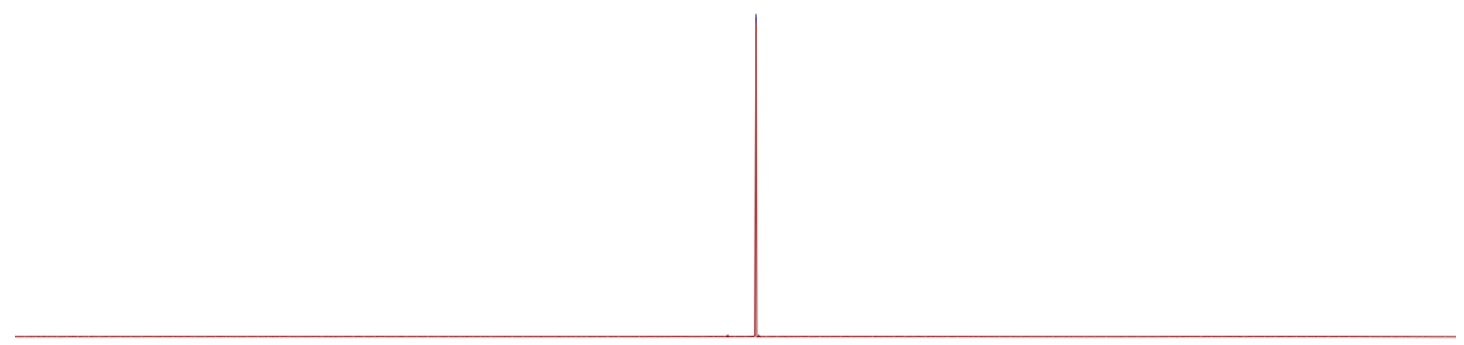

$\begin{array}{llllllllllllllllllllllllll}1 & 10 & 0 & -10 & -20 & -30 & -40 & -50 & -60 & -70 & -80 & -90 & -100 & -110 & -120 & -130 & -140 & -150 & -160 & -170 & -180 & -190 & -200 & -210 & 1\end{array}$

${ }^{13} \mathrm{C}$ NMR (100 MHz, $\left.\mathrm{CDCl}_{3}\right)$ of $7 \mathrm{e}$

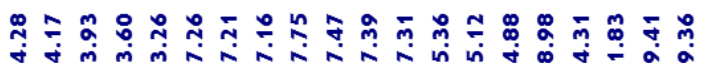

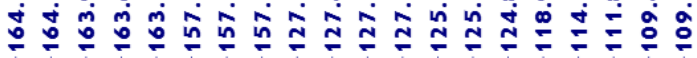

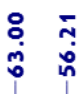

方

$\mathrm{CDCl}_{3}, 77.16$
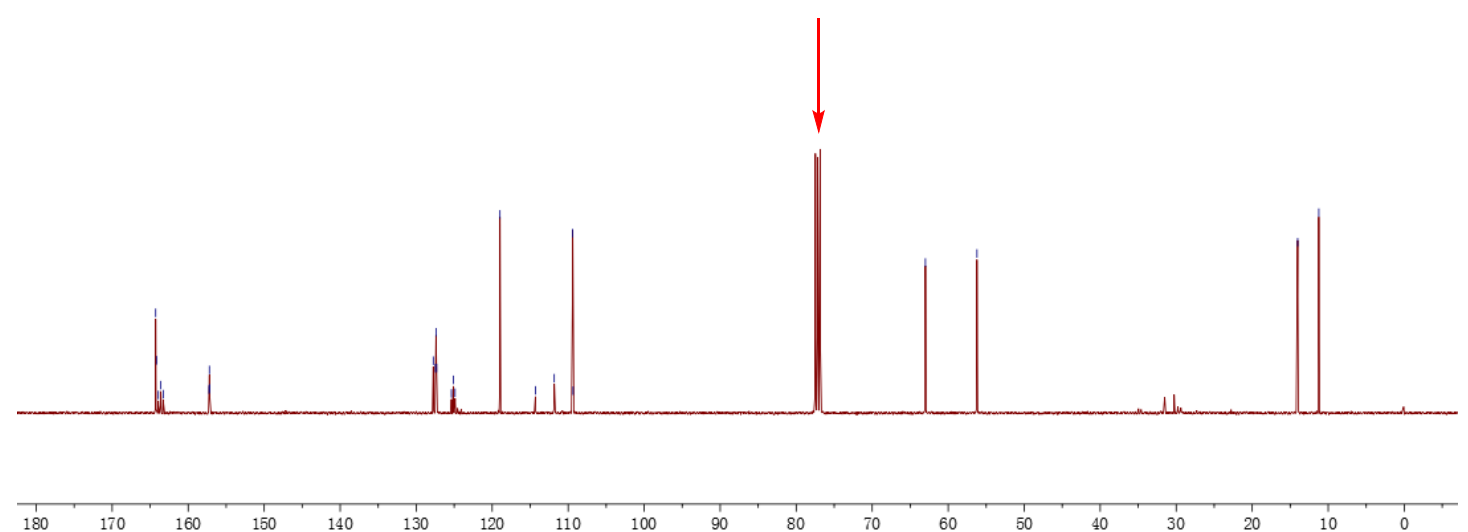

150

140

120

100

f1 (ppm) 80 
${ }^{1} \mathrm{H}$ NMR (400 MHz, $\mathrm{CDCl}_{3}$ ) of $7 f$

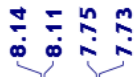

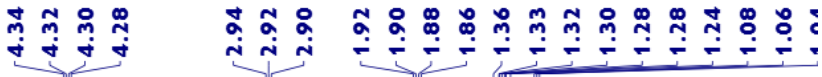
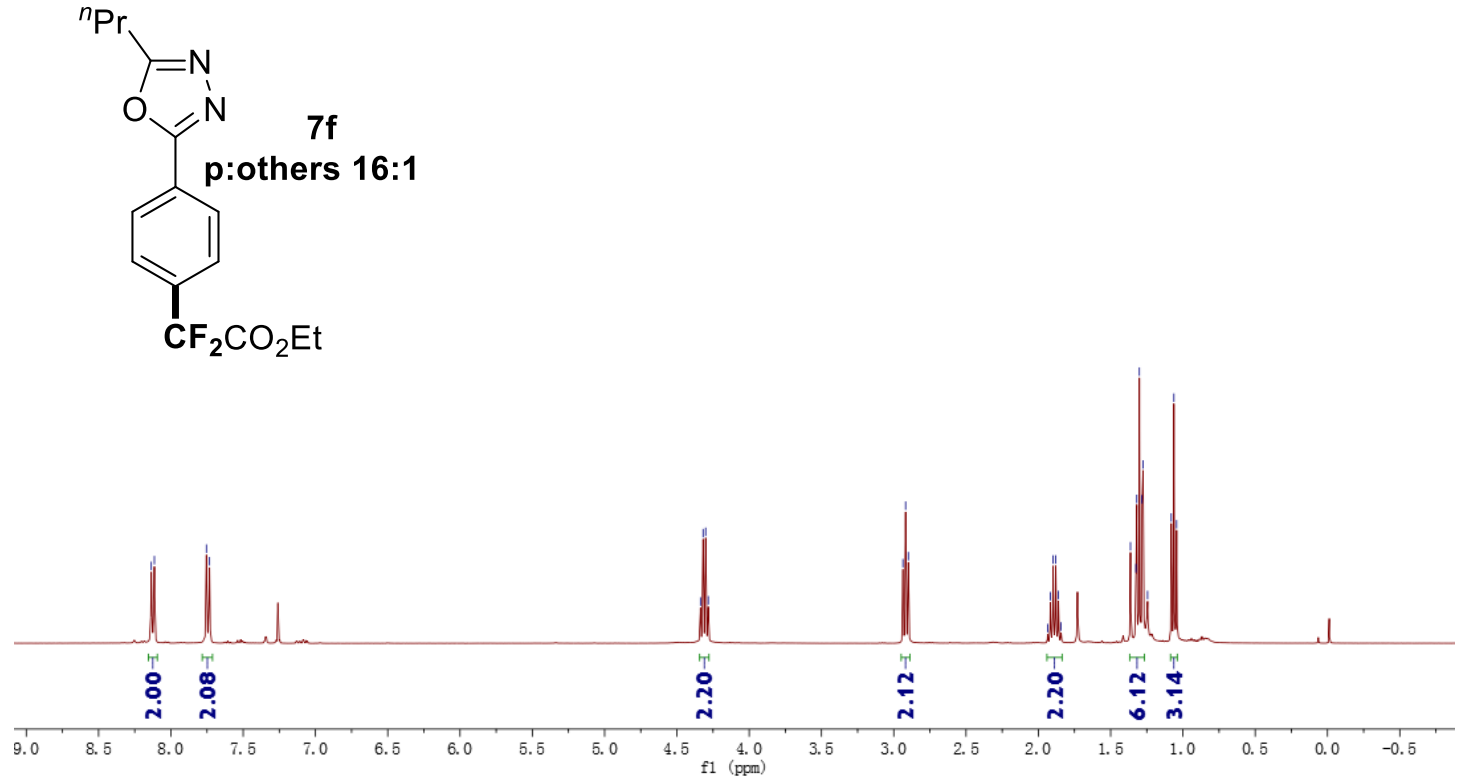

${ }^{19}$ F NMR (376 $\left.\mathrm{MHz}, \mathrm{CDCl}_{3}\right)$ of $7 \mathrm{f}$

$$
\text { รั) }
$$
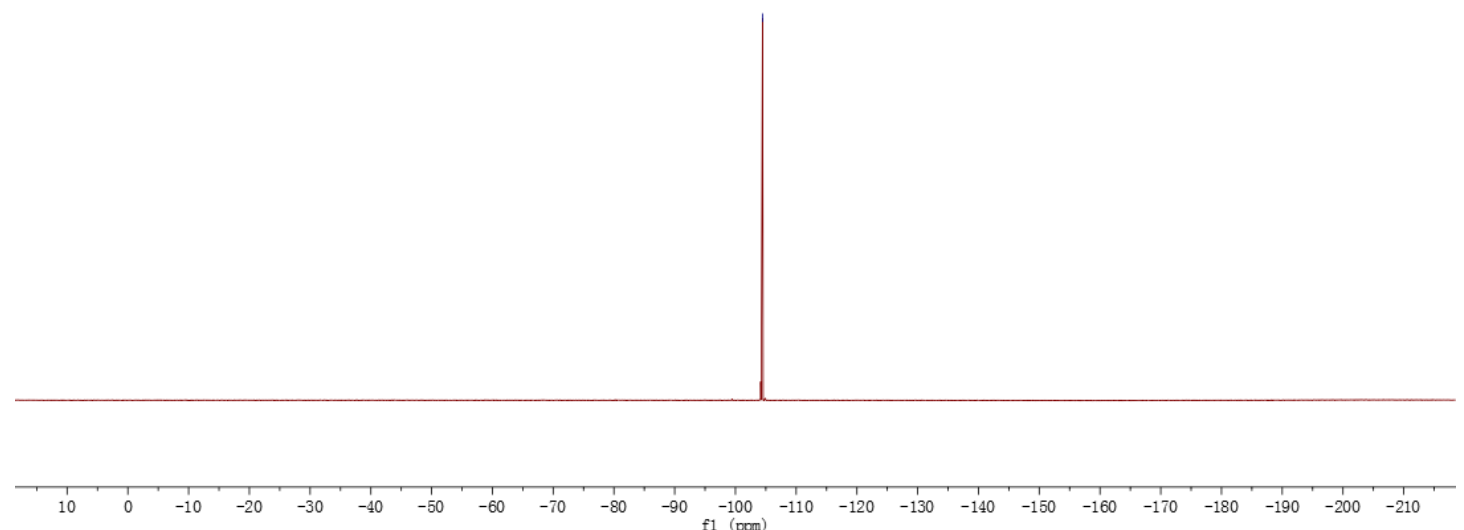
${ }^{13} \mathrm{C}$ NMR (100 MHz, $\left.\mathrm{CDCl}_{3}\right)$ of $7 f$

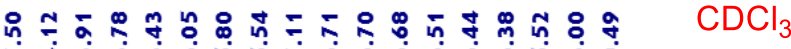

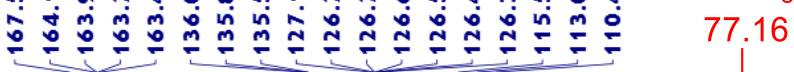
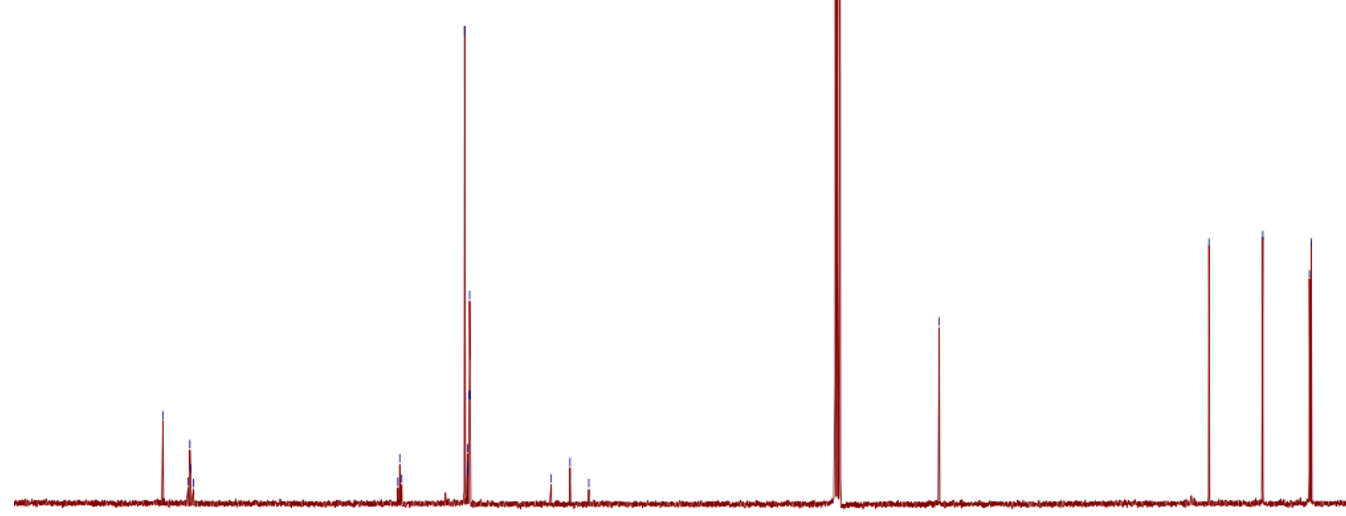

${ }^{1} \mathrm{H}$ NMR (400 MHz, $\left.\mathrm{CDCl}_{3}\right)$ of $7 \mathrm{~g}$

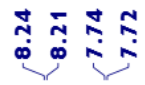
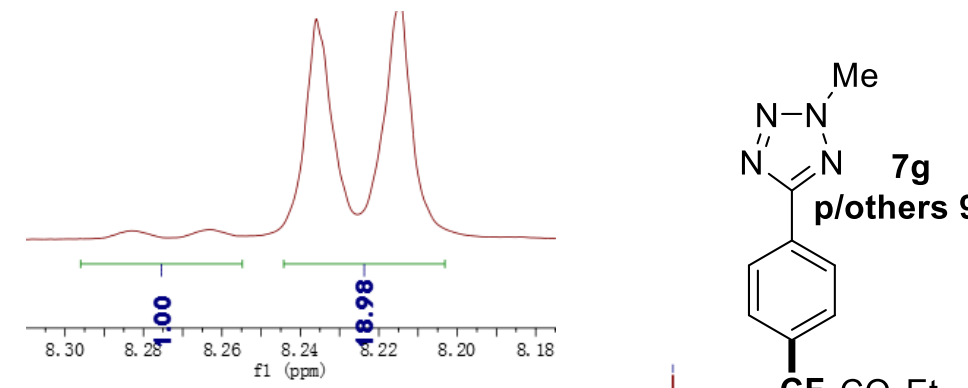

p/others 9:1

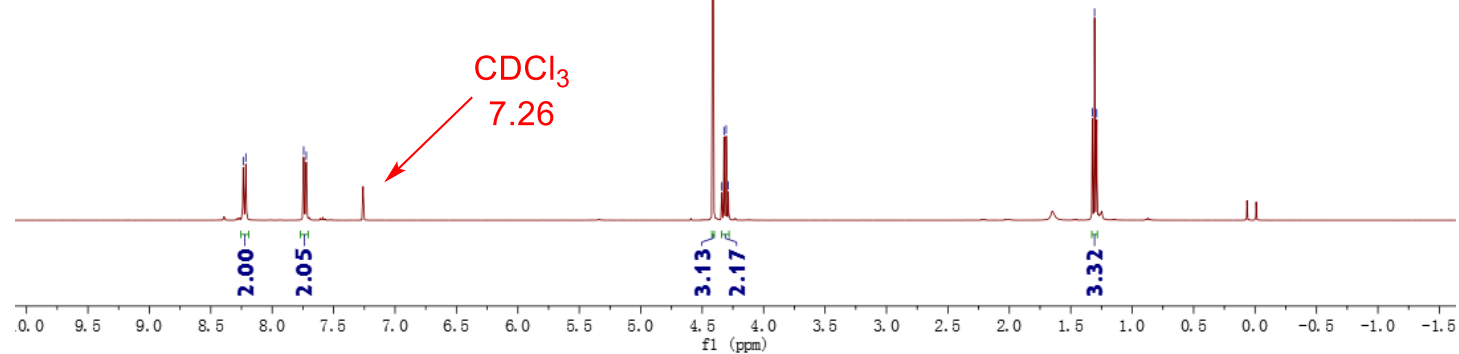


${ }^{19} \mathrm{~F}$ NMR $\left(376 \mathrm{MHz}, \mathrm{CDCl}_{3}\right)$ of $7 \mathrm{~g}$

$$
\frac{a}{i j}
$$

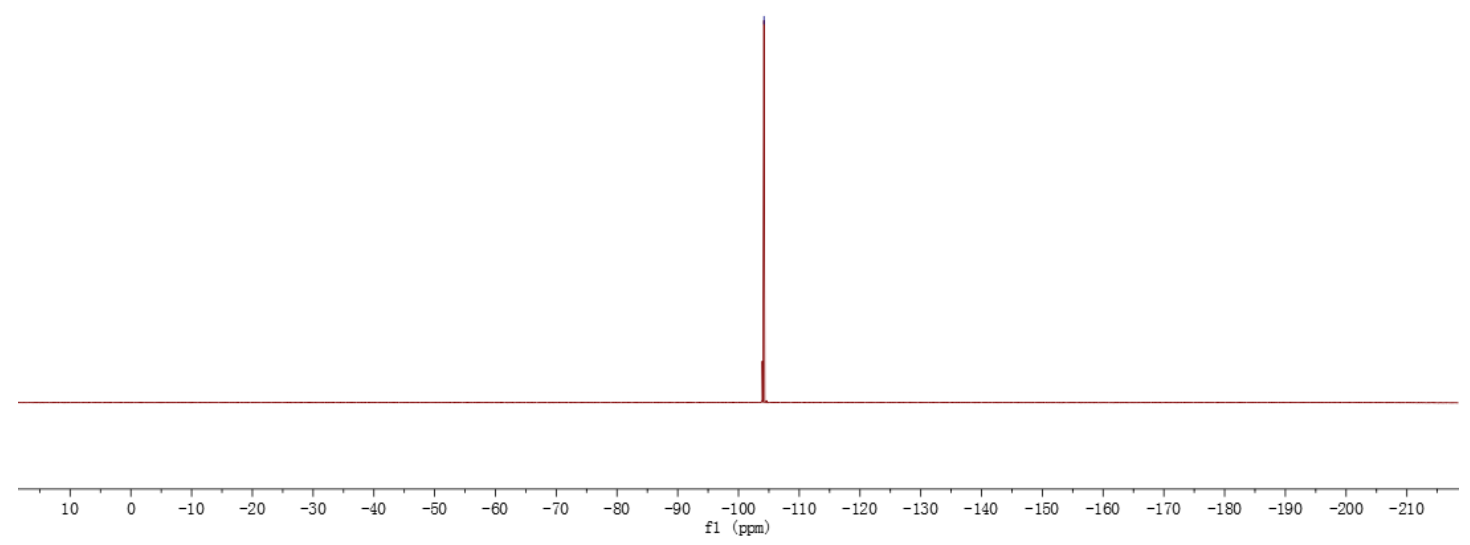

${ }^{13} \mathrm{C}$ NMR (100 $\left.\mathrm{MHz}, \mathrm{CDCl}_{3}\right)$ of $7 \mathrm{~g}$

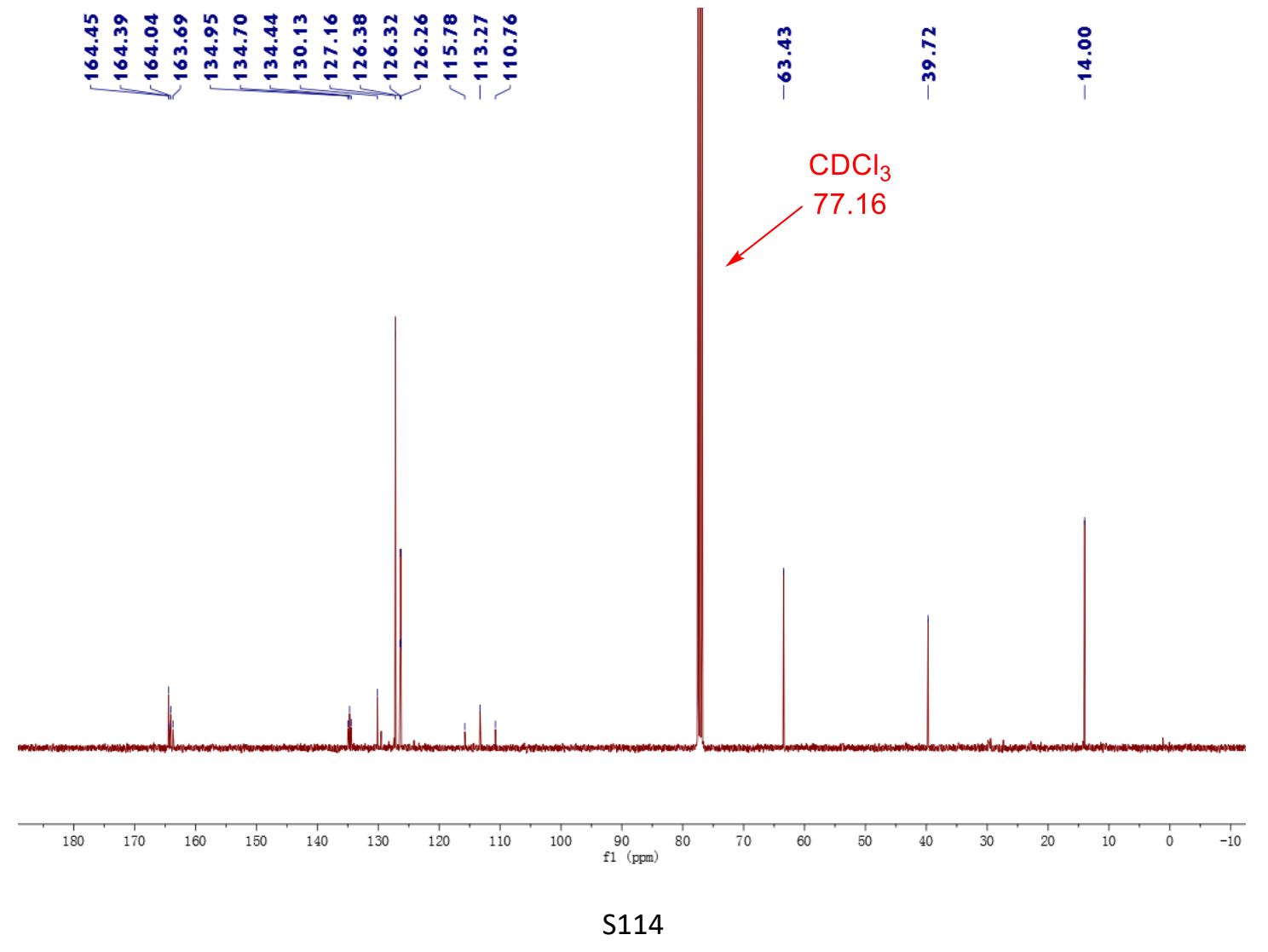


${ }^{1} \mathrm{H}$ NMR (400 MHz, CDCl 3$)$ of $7 \mathrm{~h}$

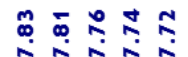<smiles>CCOC(=O)C(F)(F)c1ccc(-c2nnn(C)n2)cc1OC</smiles>

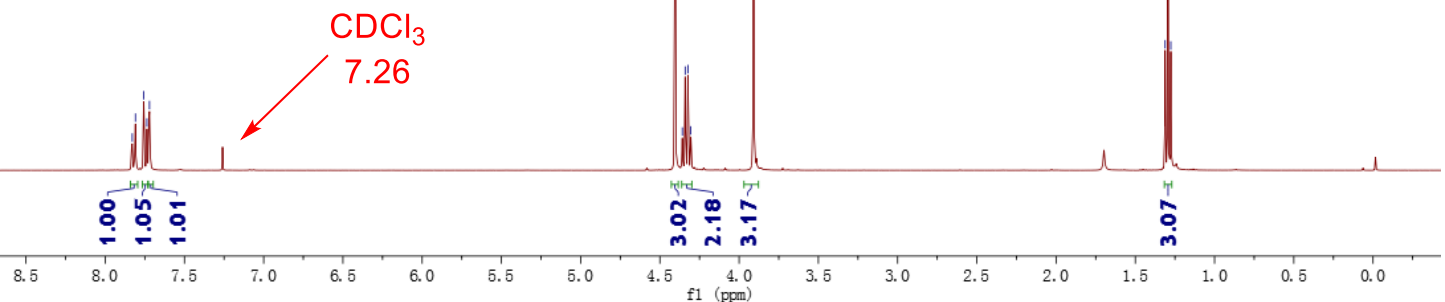

${ }^{19}$ F NMR (376 MHz, $\left.\mathrm{CDCl}_{3}\right)$ of $7 \mathrm{~h}$

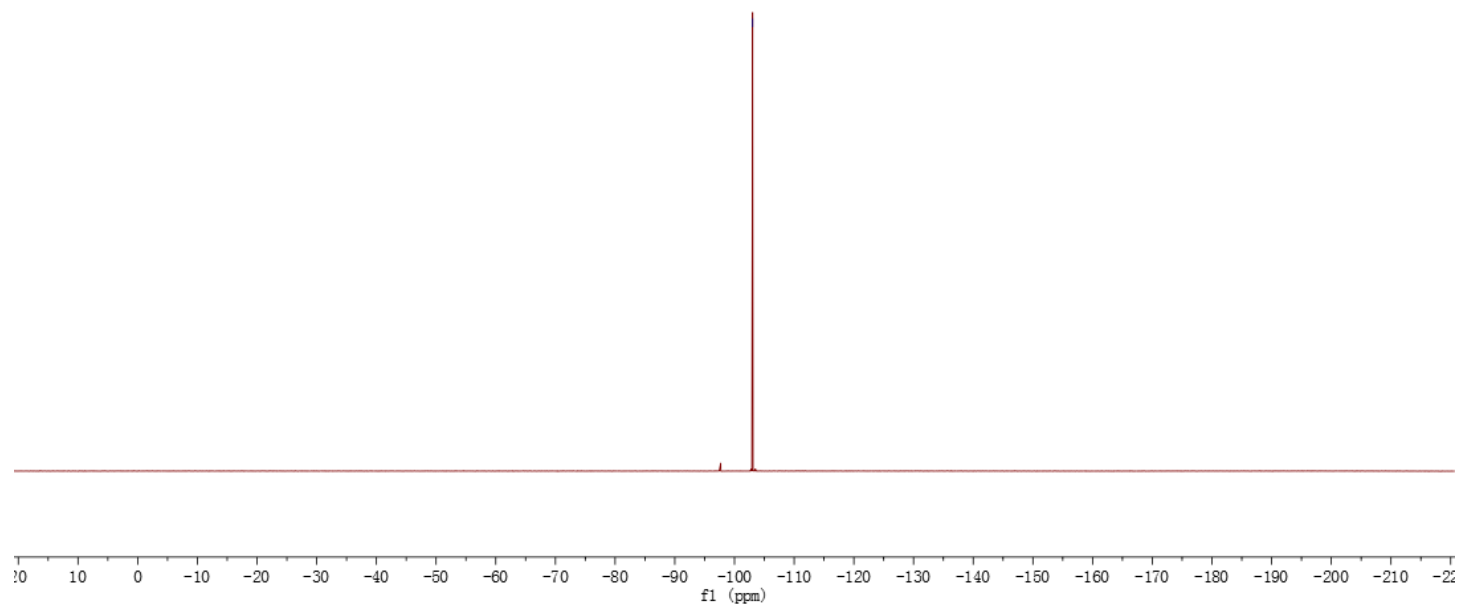


${ }^{13} \mathrm{C}$ NMR (100 MHz, $\left.\mathrm{CDCl}_{3}\right)$ of $7 \mathrm{~h}$

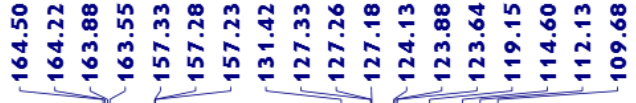

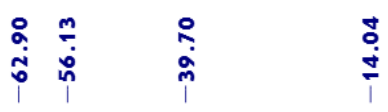

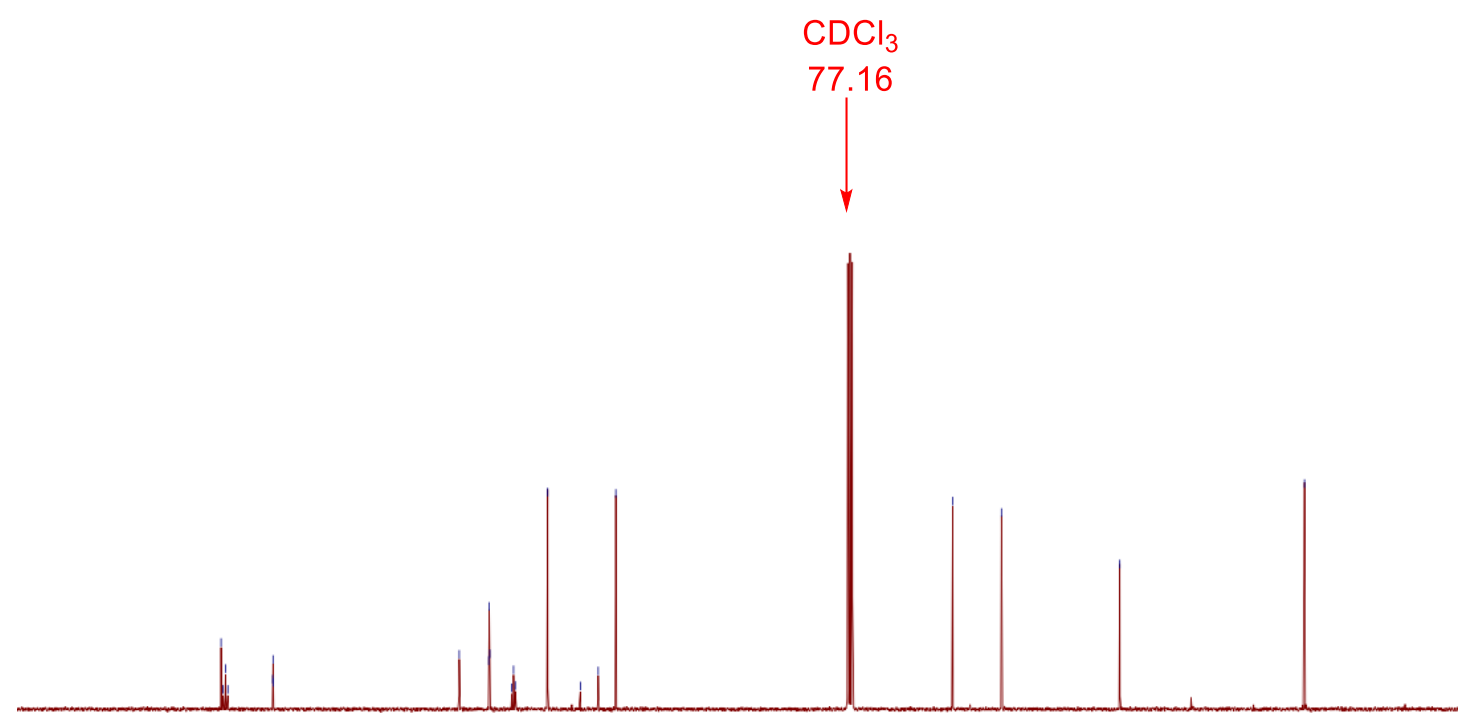

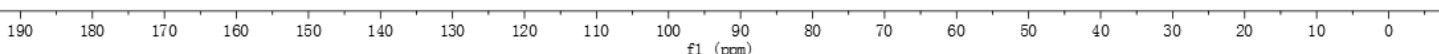

${ }^{1} \mathrm{H}$ NMR (400 MHz, CDCl $)$ of 9a

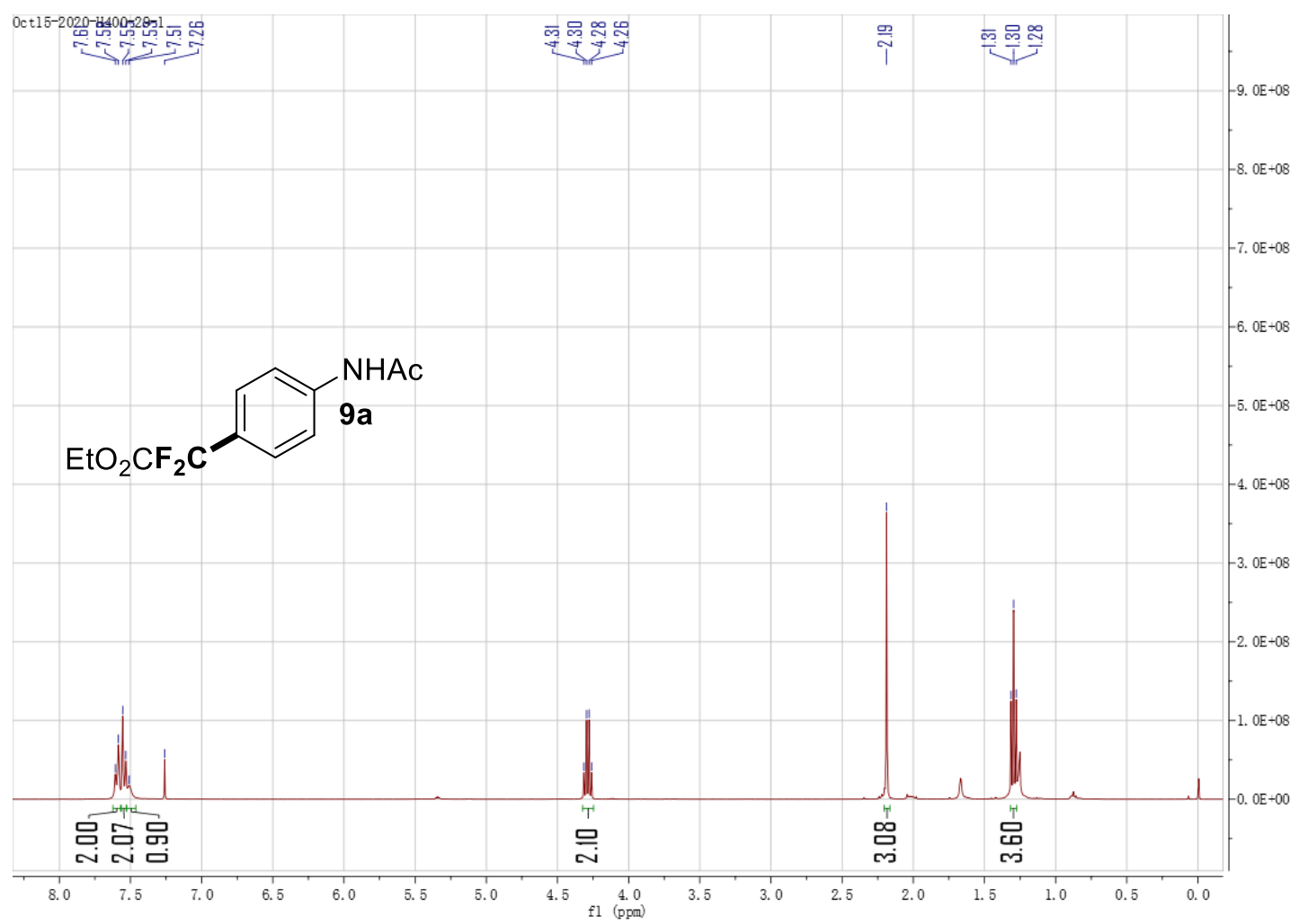


${ }^{19}$ F NMR (376 $\left.\mathrm{MHz}, \mathrm{CDCl}_{3}\right)$ of $9 \mathrm{a}$

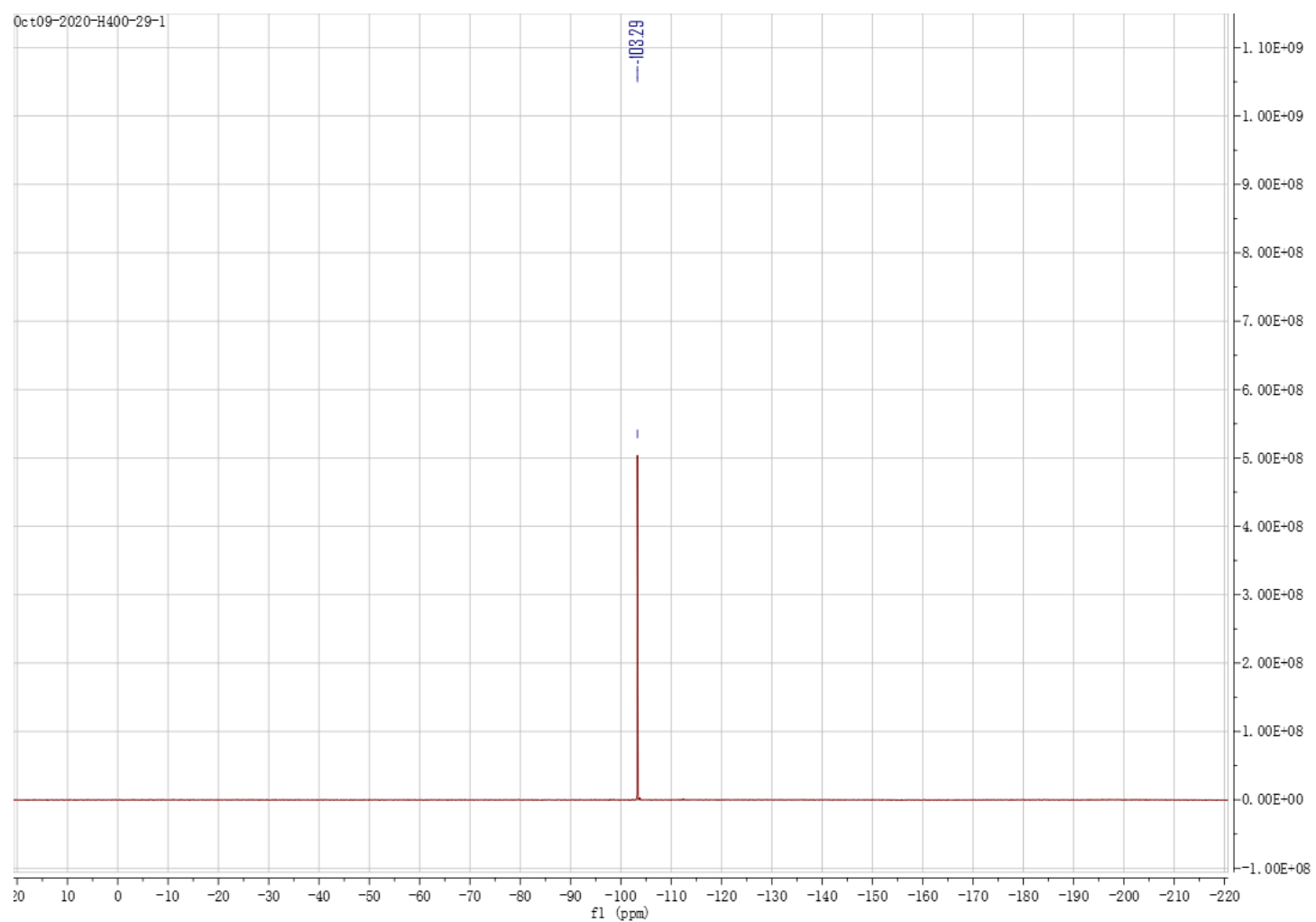

${ }^{13} \mathrm{C}$ NMR (100 $\left.\mathrm{MHz}, \mathrm{CDCl}_{3}\right)$ of 9a

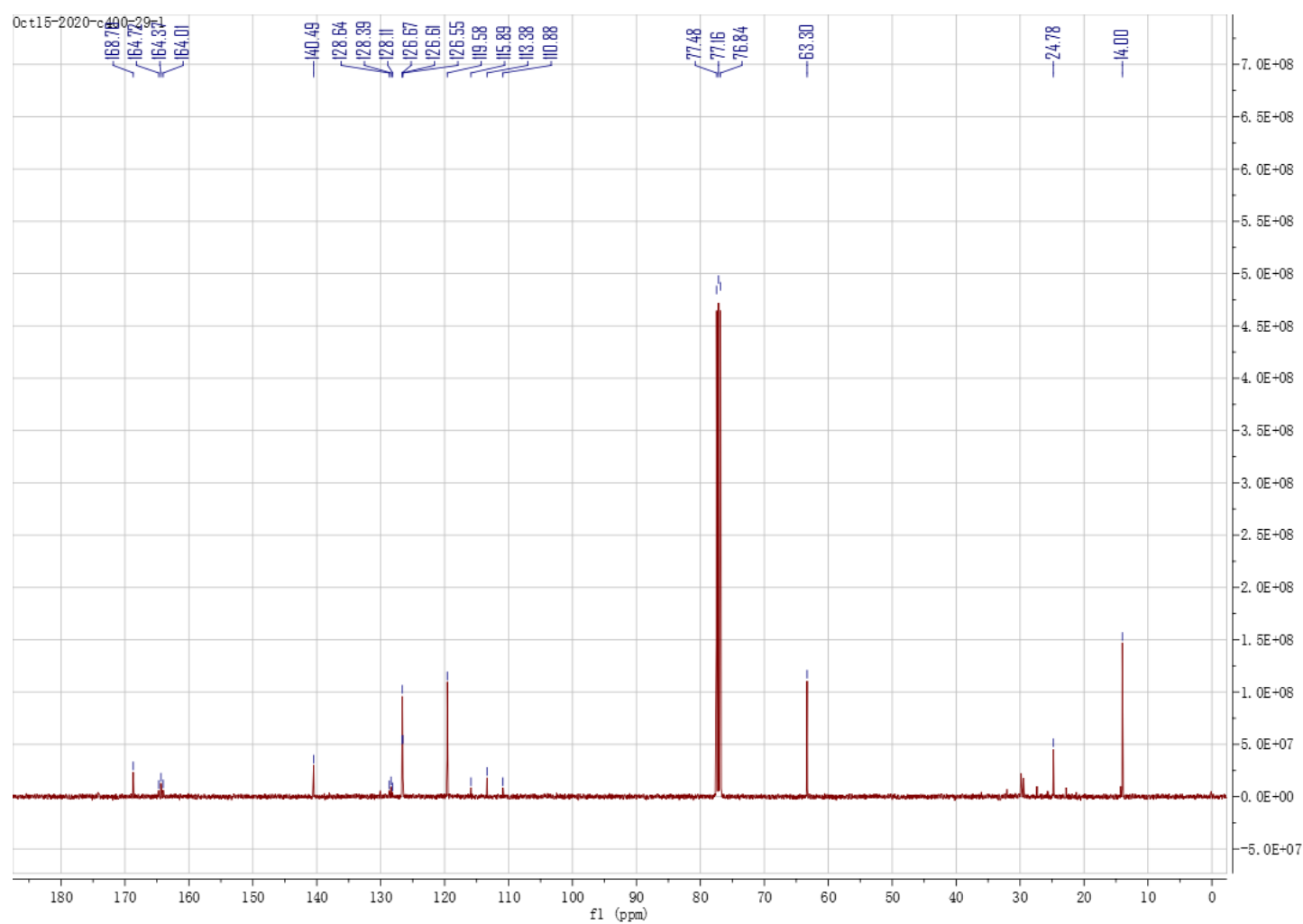


${ }^{1} \mathrm{H}$ NMR (400 MHz, CDCl 3 ) of $9 \mathrm{~b}$

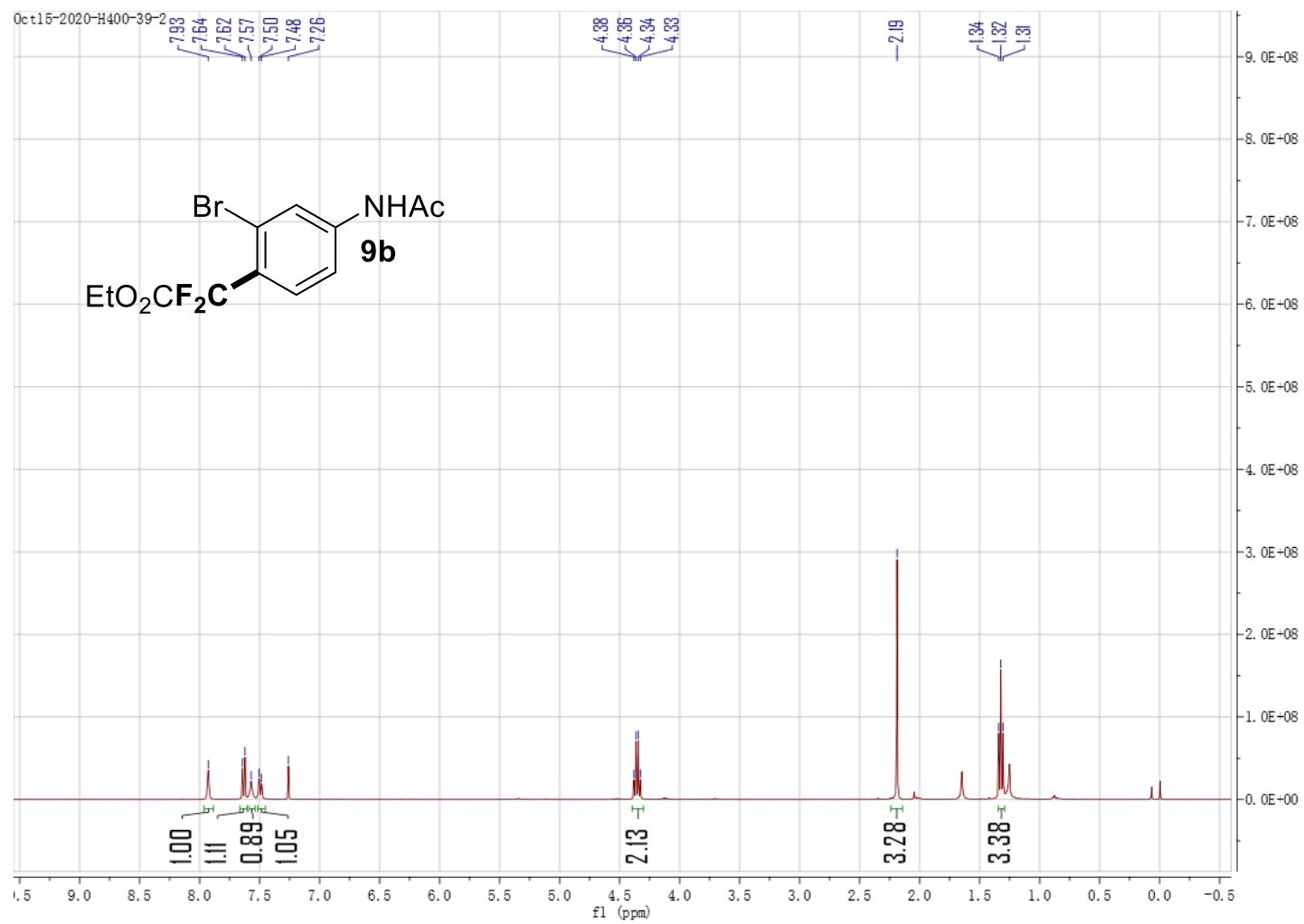

${ }^{19}$ F NMR (376 MHz, $\left.\mathrm{CDCl}_{3}\right)$ of 9b

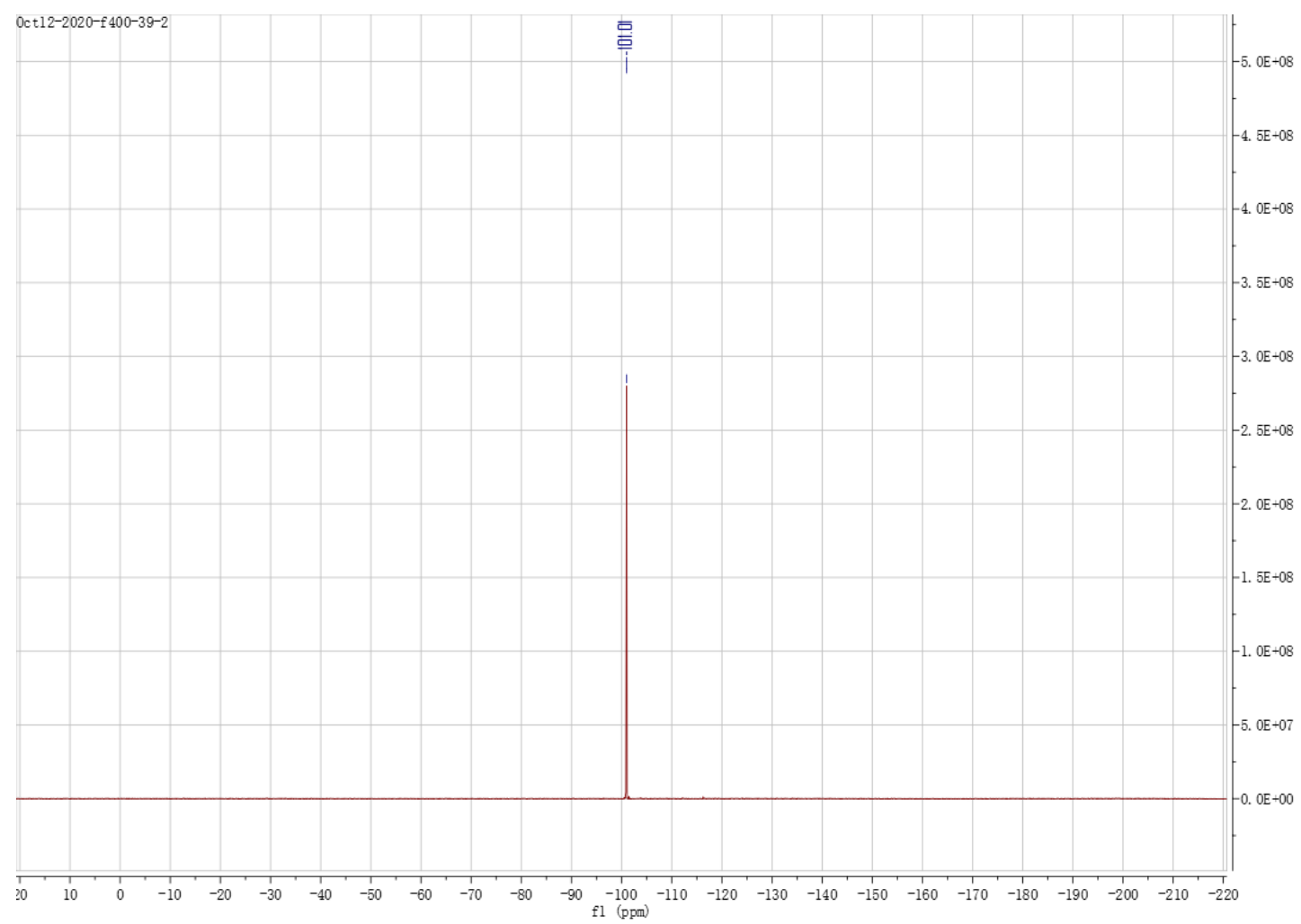


${ }^{13} \mathrm{C}$ NMR (100 MHz, $\left.\mathrm{CDCl}_{3}\right)$ of $9 \mathrm{~b}$

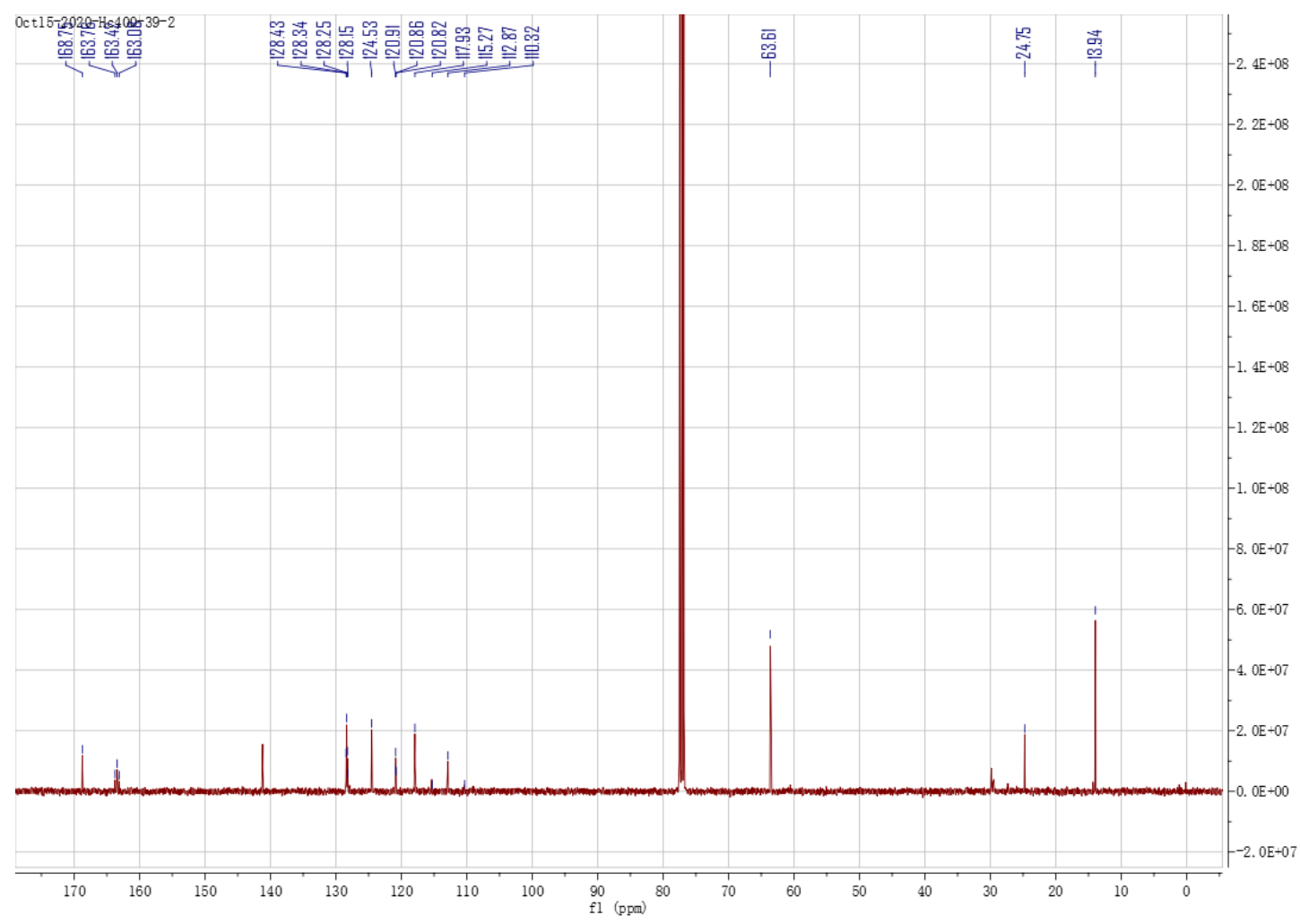

${ }^{1} \mathrm{H}$ NMR (400 MHz, $\left.\mathrm{CDCl}_{3}\right)$ of 9c

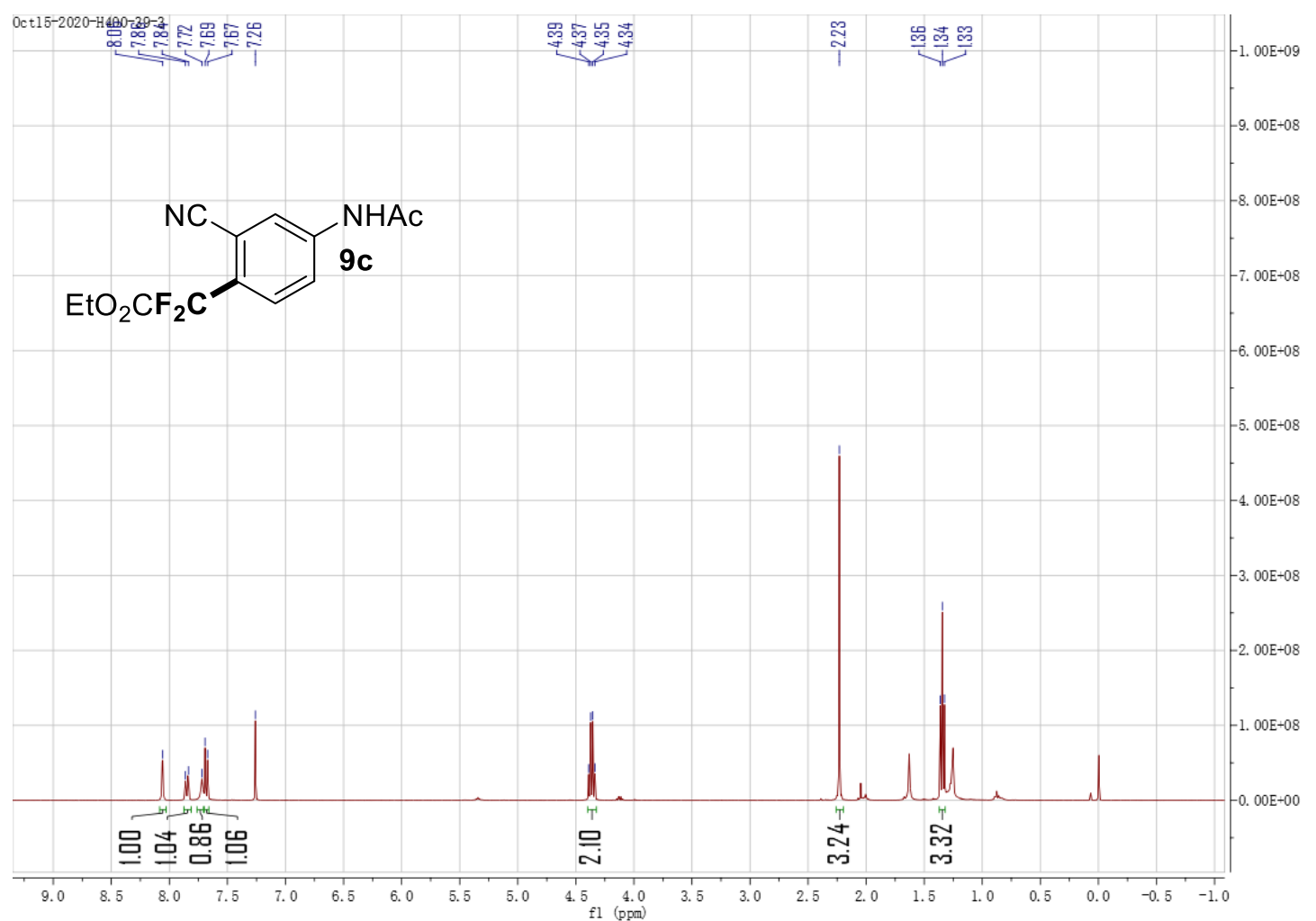



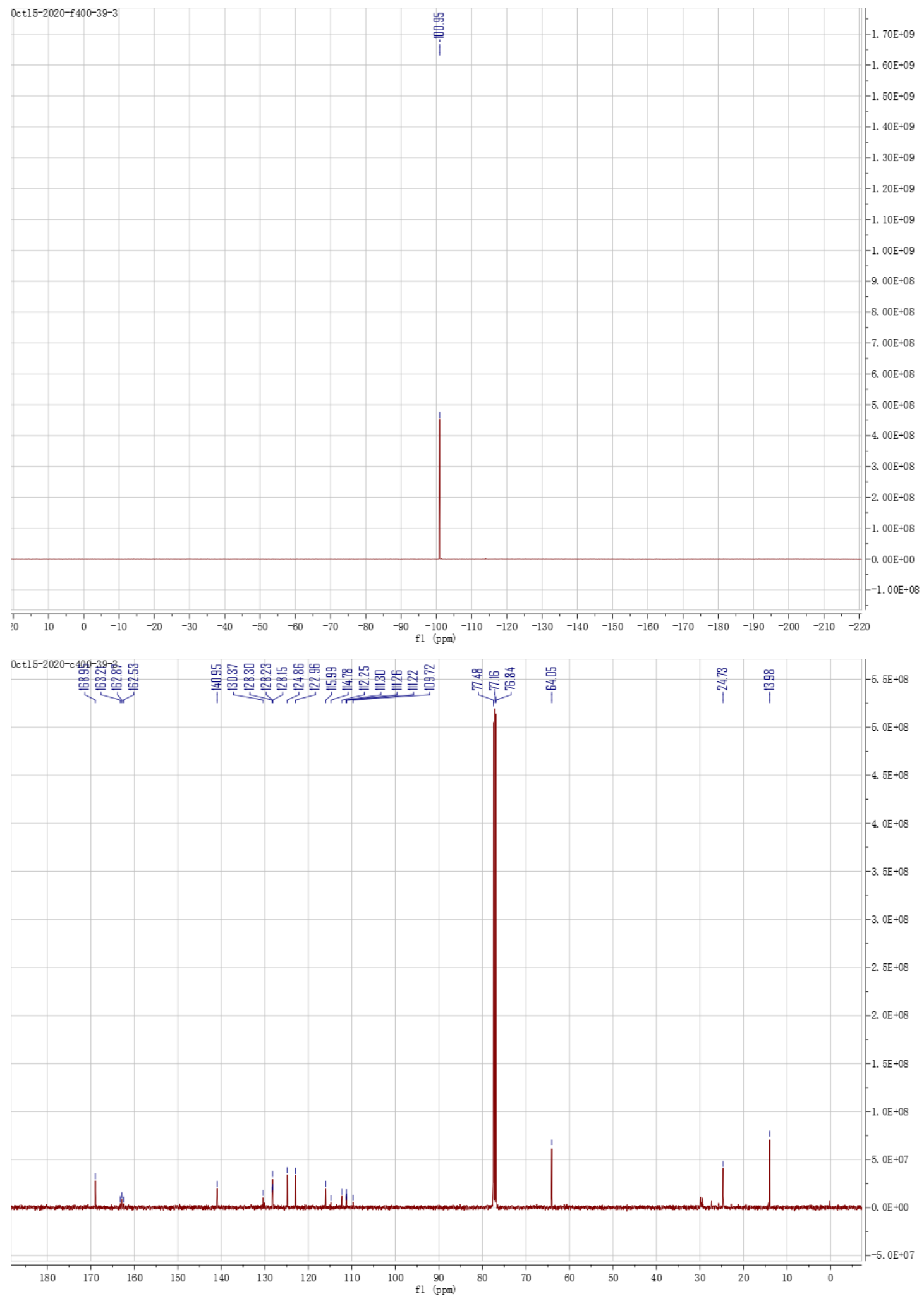


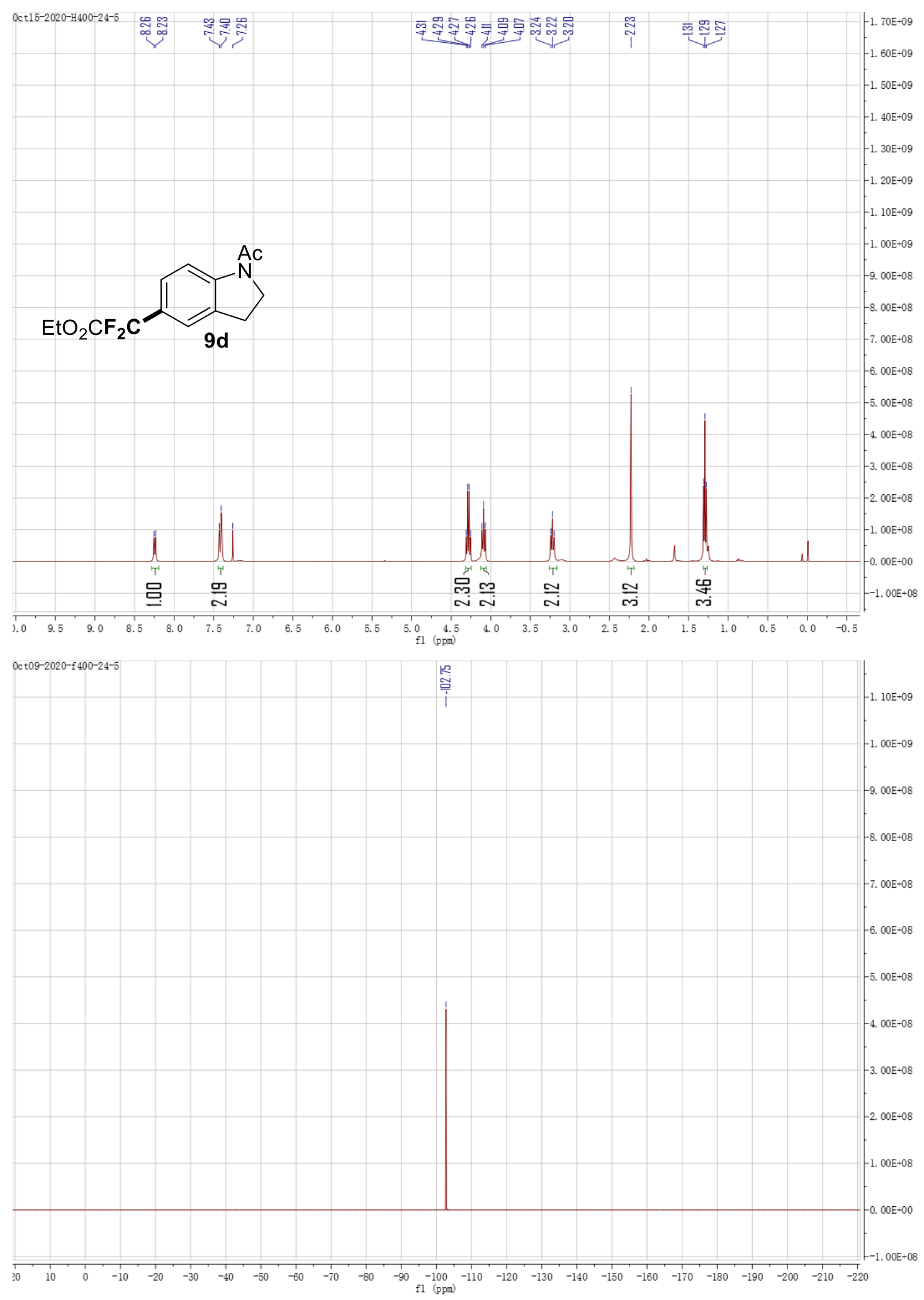




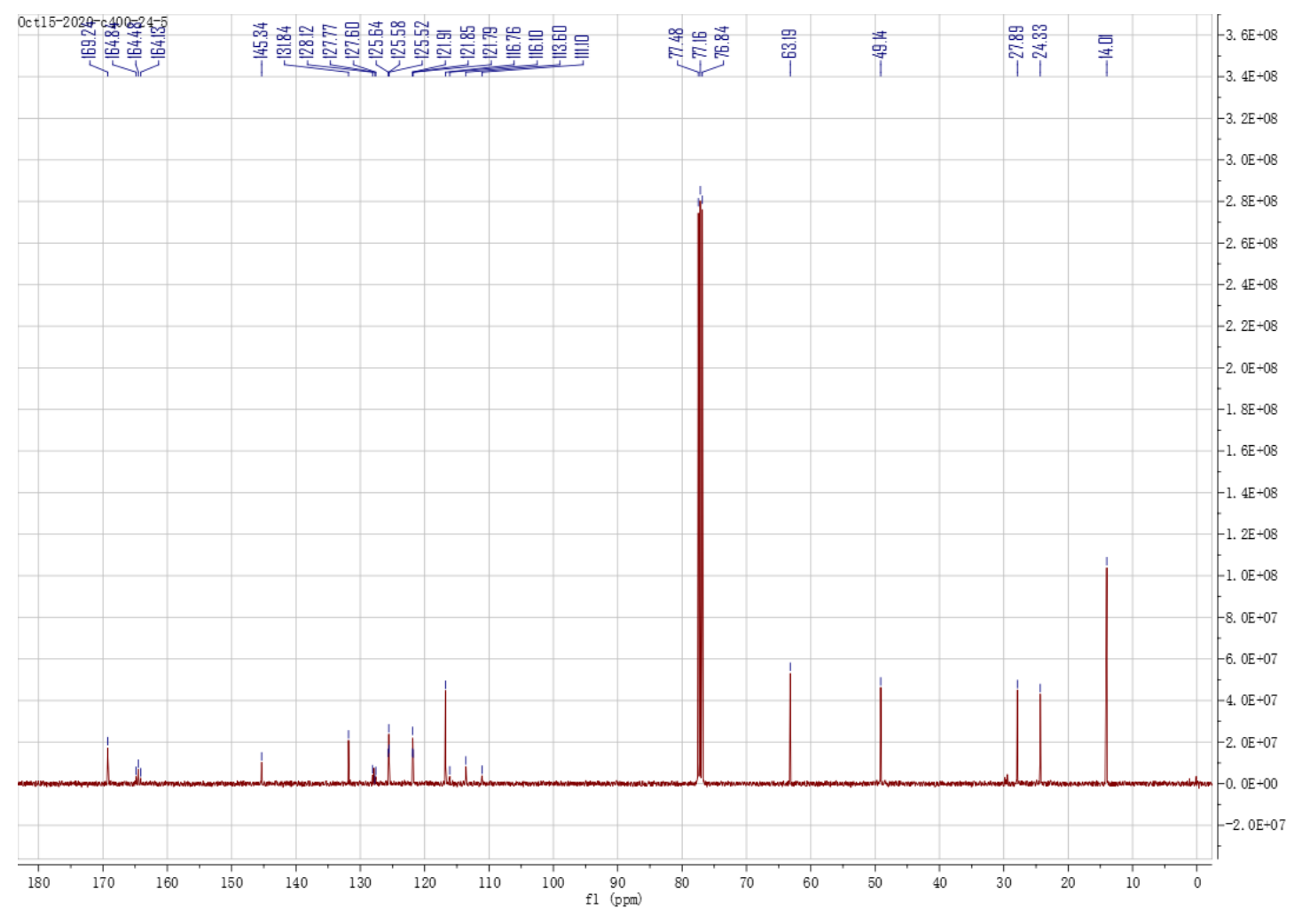

${ }^{1} \mathrm{H}$ NMR (400 MHz, $\left.\mathrm{CDCl}_{3}\right)$ of 13

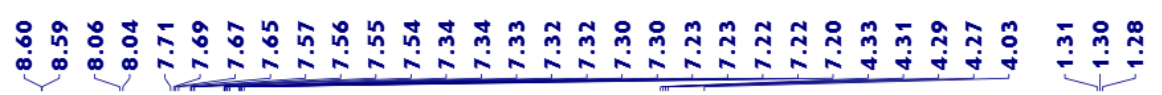
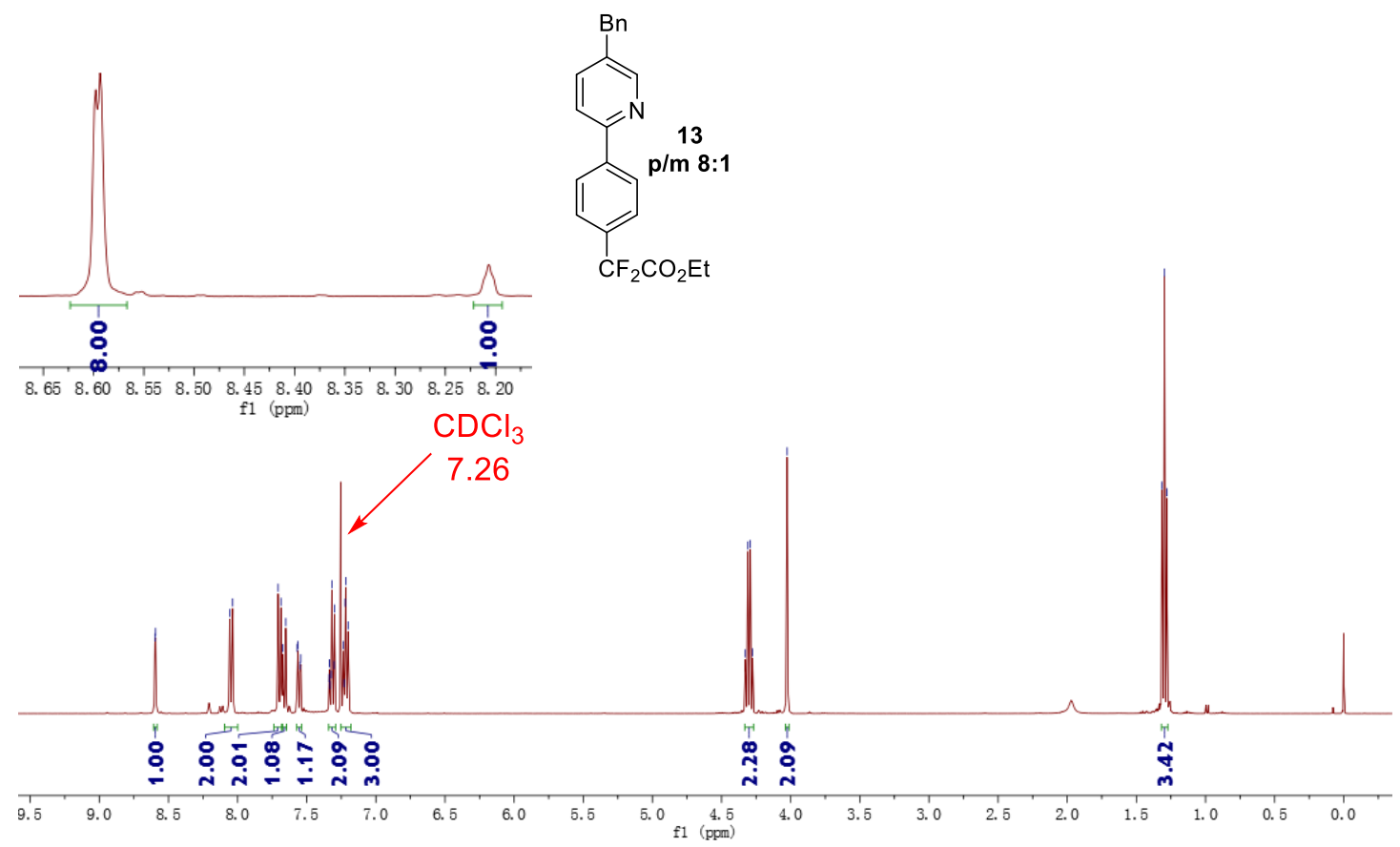
${ }^{19}$ F NMR (376 MHz, CDCl $)$ of 11

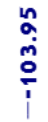

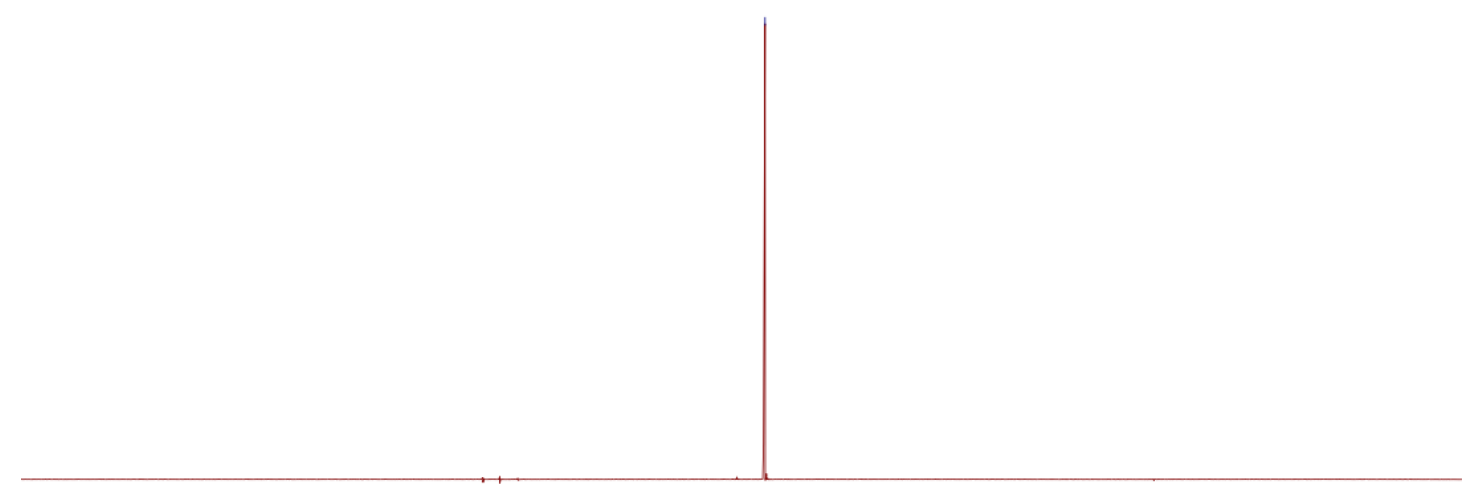

\begin{tabular}{llllllllllllllllllllllllllllll}
10 & 10 & 0 & -10 & -20 & -30 & -40 & -50 & -60 & -70 & -80 & -90 & -100 & -110 & -120 & -130 & -140 & -150 & -160 & -170 & -180 & -190 & -200 & -210 & -22 \\
\hline
\end{tabular}

${ }^{13} \mathrm{C}$ NMR (100 MHz, $\left.\mathrm{CDCl}_{3}\right)$ of 13

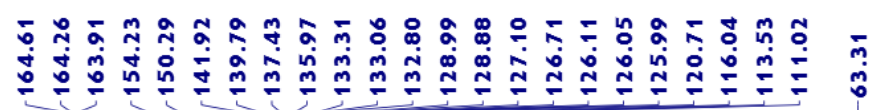

$\stackrel{\infty}{\infty} \underset{\infty}{\infty} \quad \stackrel{\circ}{i}$

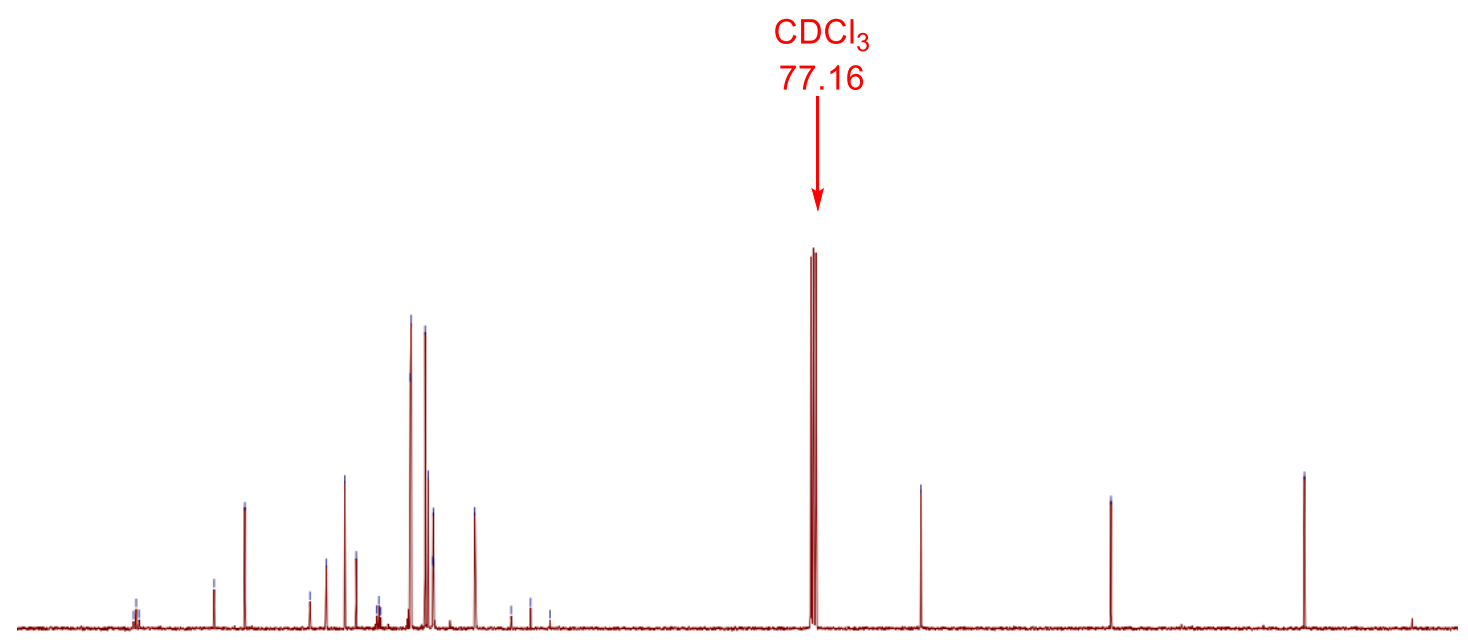

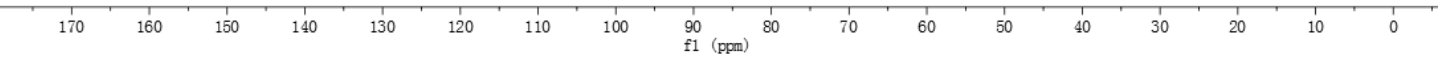


${ }^{1} \mathrm{H}$ NMR (400 MHz, $\left.\mathrm{CDCl}_{3}\right)$ of $5 \mathrm{~s}$

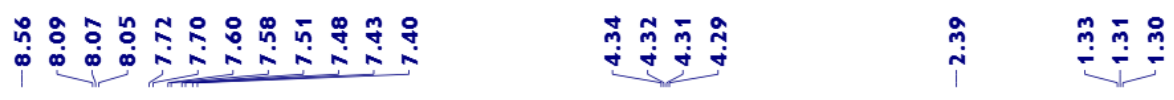

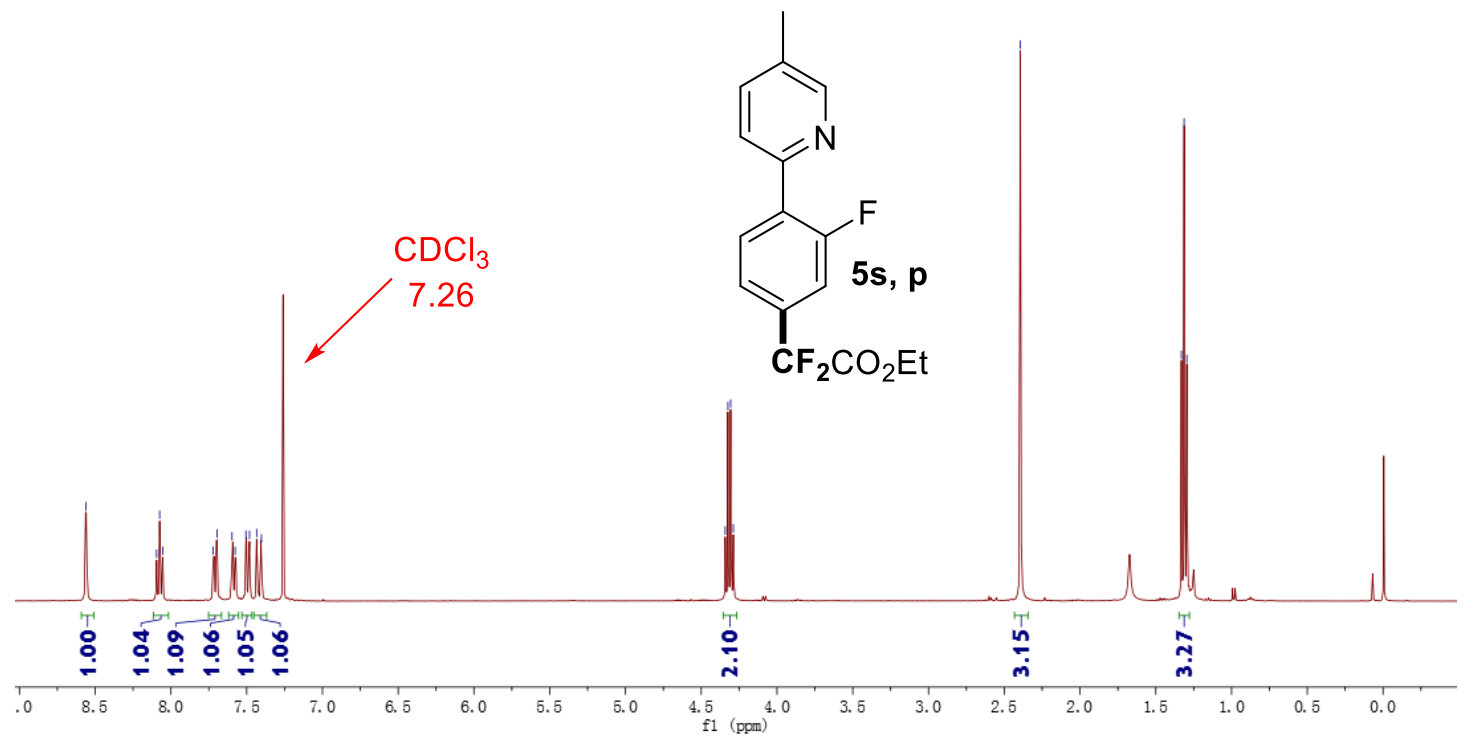

${ }^{19} \mathrm{~F}$ NMR (376 MHz, $\left.\mathrm{CDCl}_{3}\right)$ of $5 \mathrm{~s}$

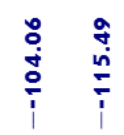

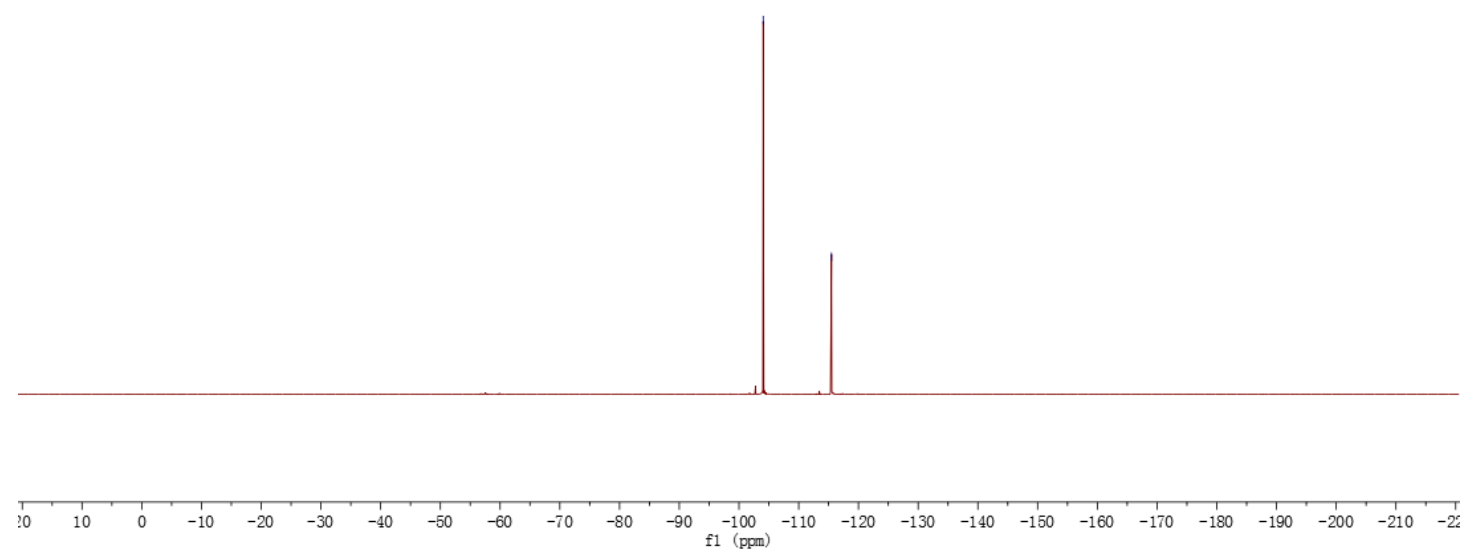


${ }^{13} \mathrm{C}$ NMR (100 MHz, $\left.\mathrm{CDCl}_{3}\right)$ of $5 \mathrm{~s}$

-

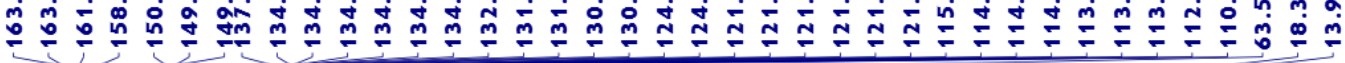
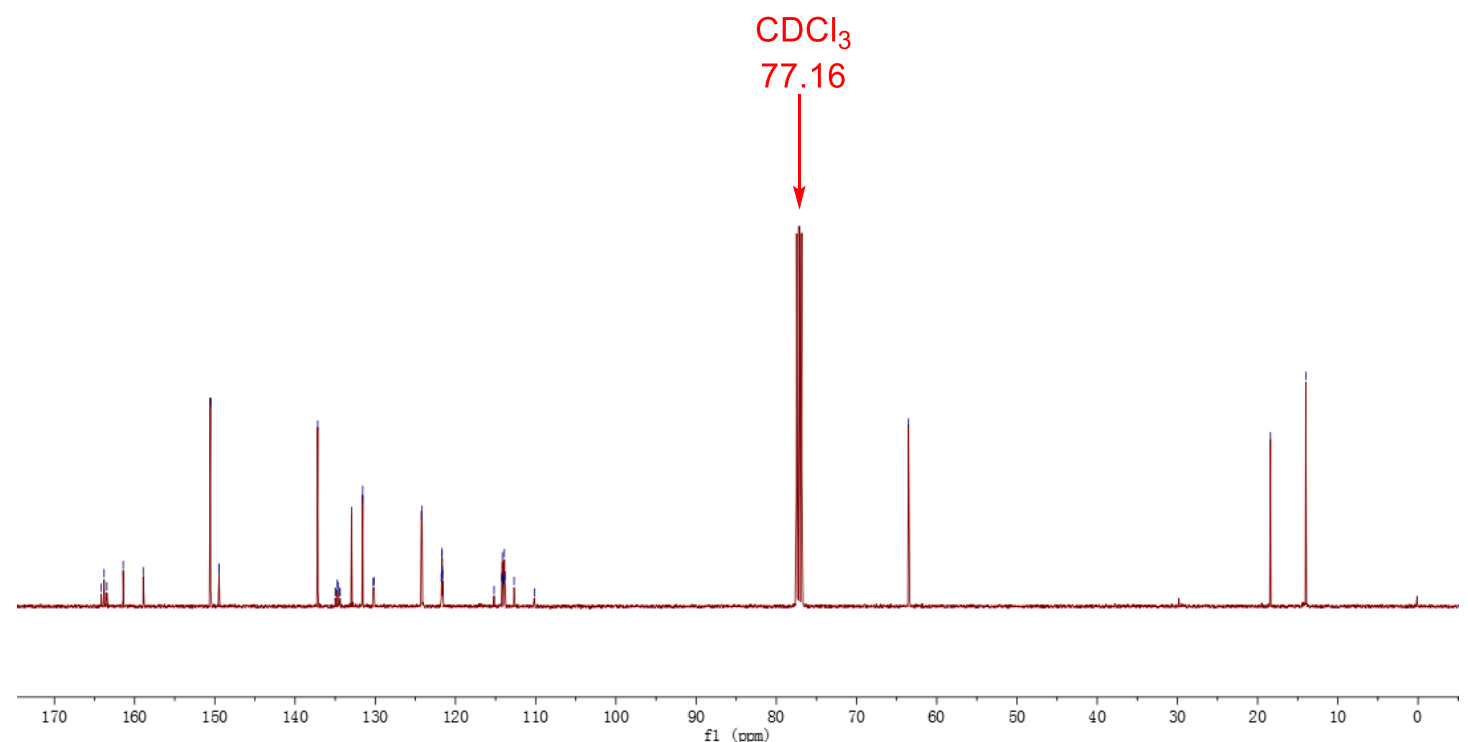

${ }^{1} \mathrm{H}$ NMR (400 MHz, $\left.\mathrm{CDCl}_{3}\right)$ of $5 p$

กุ广

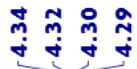

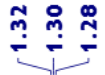<smiles>CCOC(=O)C(F)(F)c1ccc(-c2nc(Cl)cc(Cl)n2)cc1</smiles>

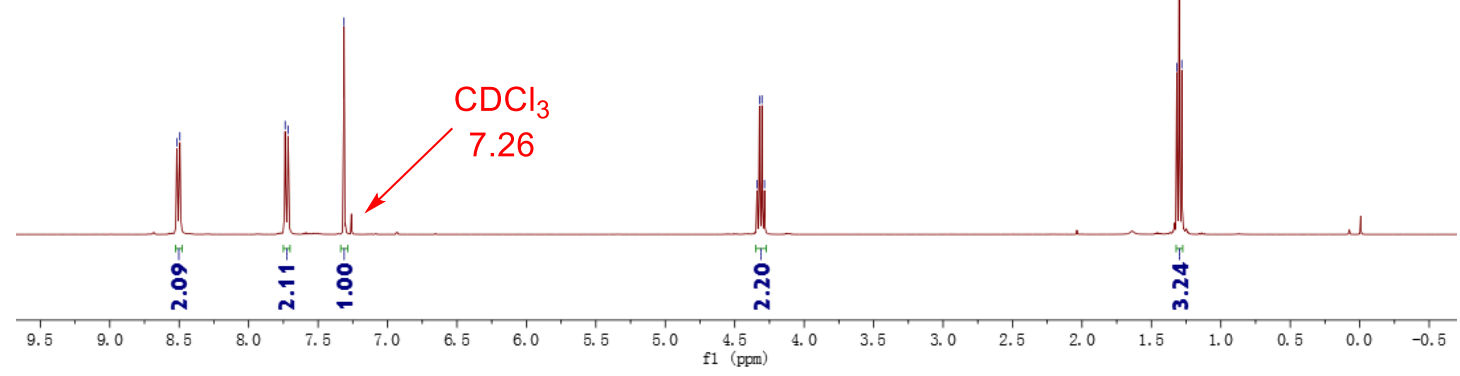

${ }^{19}$ F NMR (376 MHz, $\left.\mathrm{CDCl}_{3}\right)$ of $5 p$ 


\section{$\stackrel{\substack{m \\ \dot{0}}}{i}$}

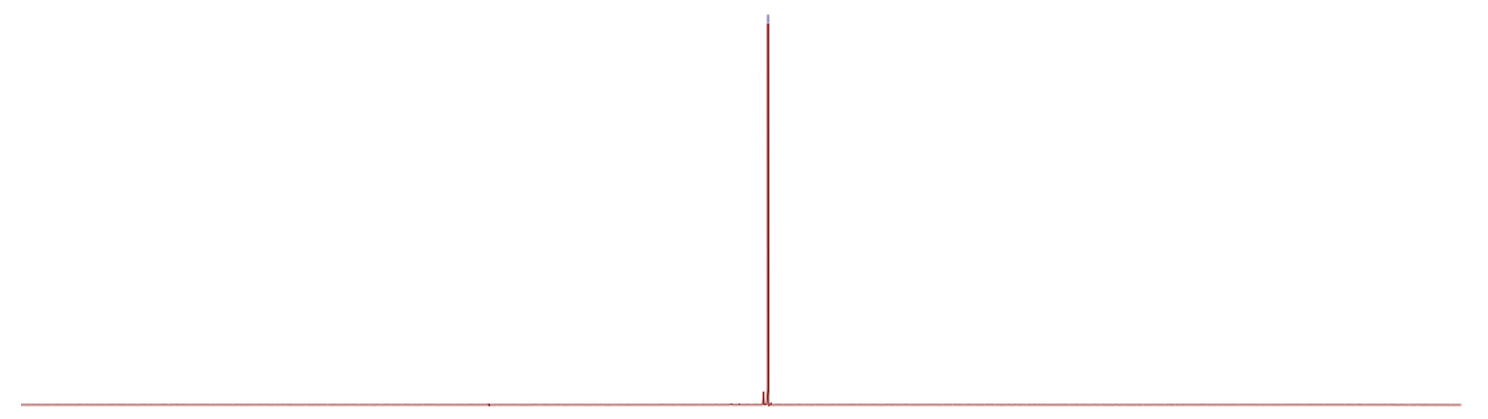

\begin{tabular}{rllllllllllllllllllllllllll}
\hline 10 & 0 & -10 & -20 & -30 & -40 & -50 & -60 & -70 & -80 & -90 & -100 & -110 & -120 & -130 & -140 & -150 & -160 & -170 & -180 & -190 & -200 & -210 & 1
\end{tabular}

${ }^{13} \mathrm{C}$ NMR (100 MHz, $\mathrm{CDCl}_{3}$ ) of 5p

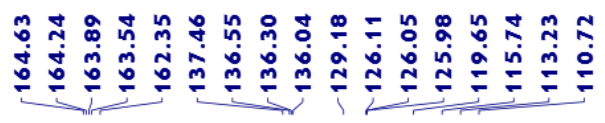

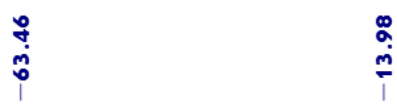

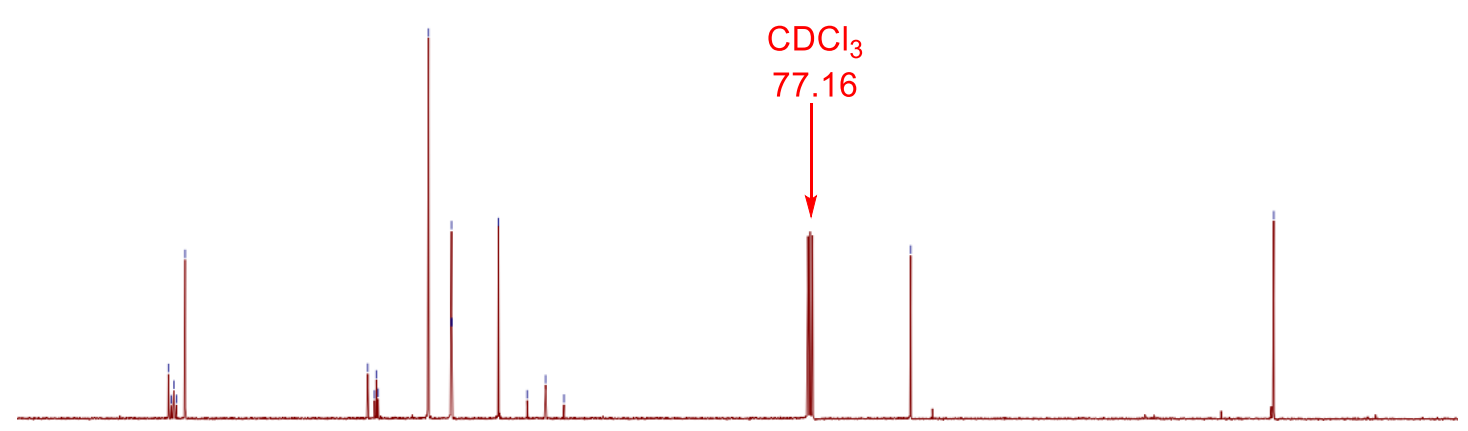

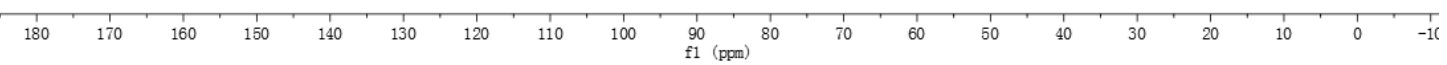


${ }^{1} \mathrm{H}$ NMR (400 MHz, $\left.\mathrm{CDCl}_{3}\right)$ of $5 q$

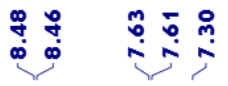

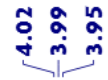

$\stackrel{m}{i}$<smiles>OCC(F)(F)c1ccc(-c2nc(Cl)cc(Cl)n2)cc1</smiles>

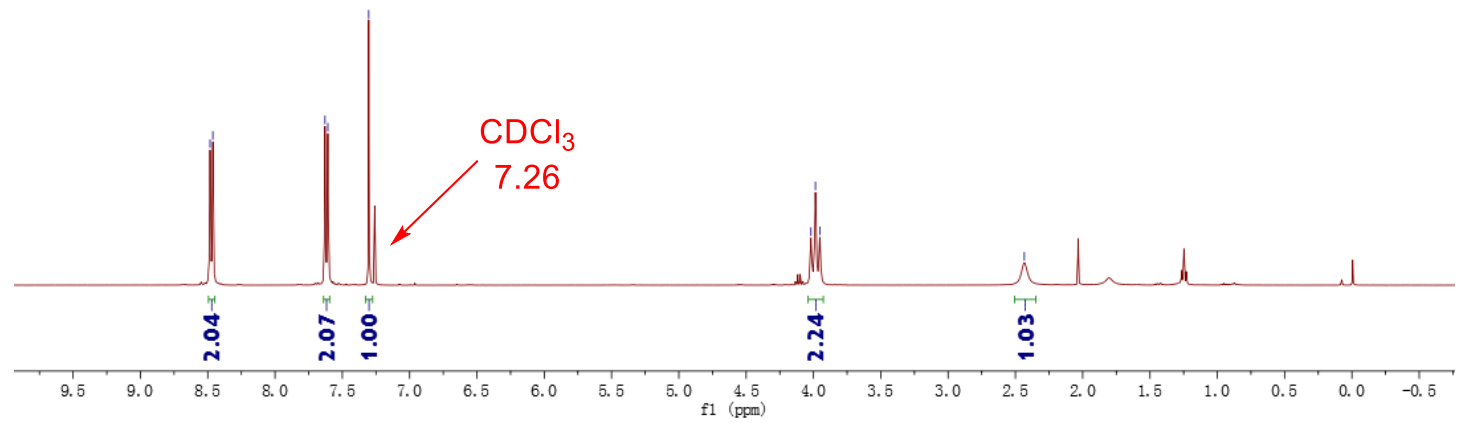

${ }^{19} \mathrm{~F}$ NMR (376 MHz, $\left.\mathrm{CDCl}_{3}\right)$ of $5 q$

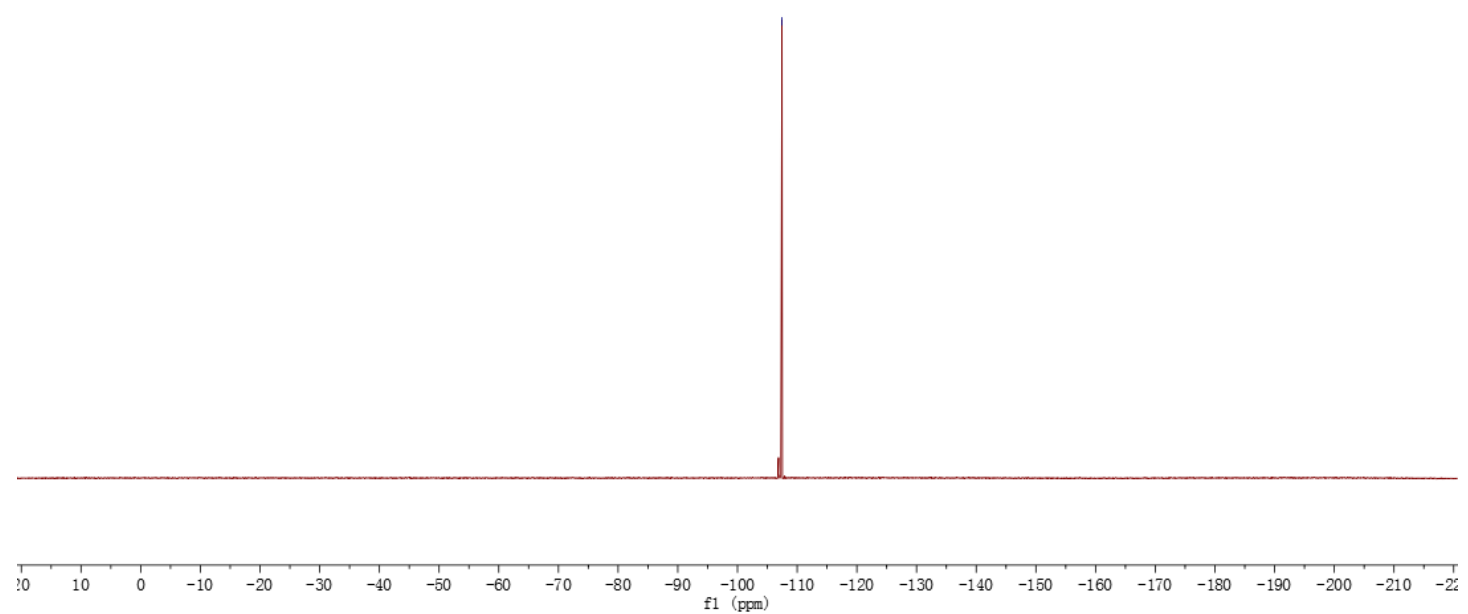


${ }^{13} \mathrm{C}$ NMR (100 $\left.\mathrm{MHz}, \mathrm{CDCl}_{3}\right)$ of $5 \mathrm{q}$

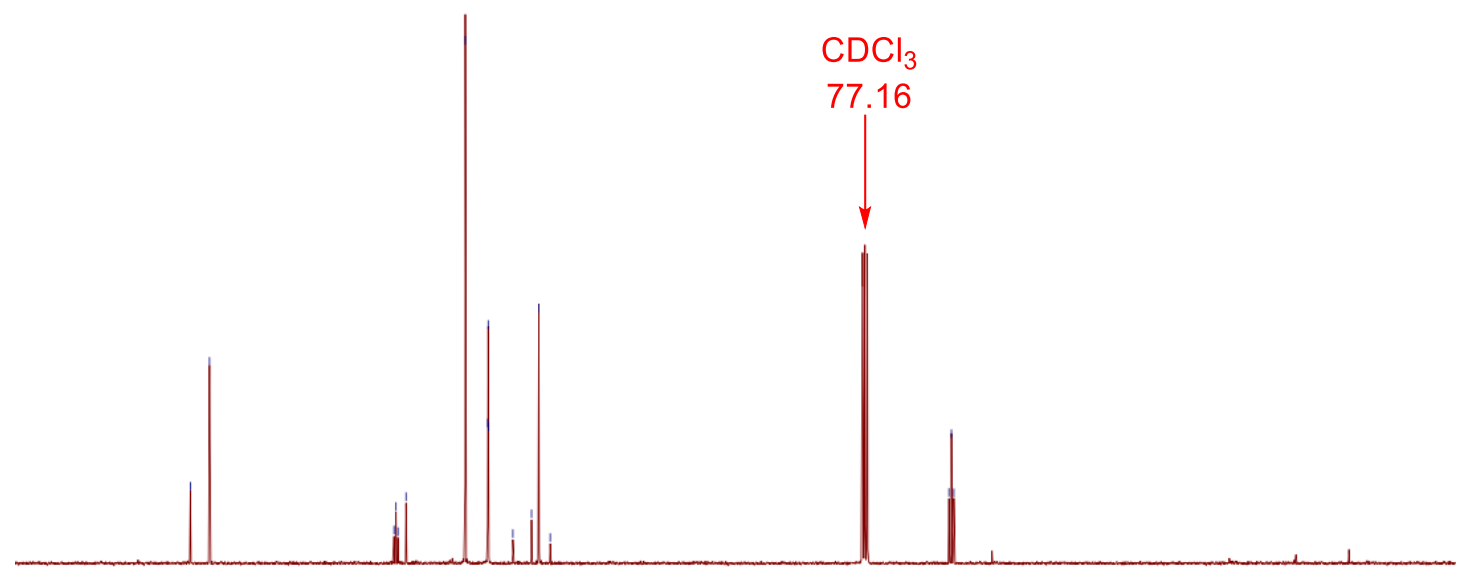

${ }^{1} \mathrm{H}$ NMR (400 MHz, $\left.\mathrm{CDCl}_{3}\right)$ of $5 \mathrm{r}$

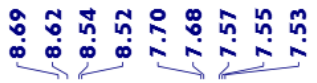

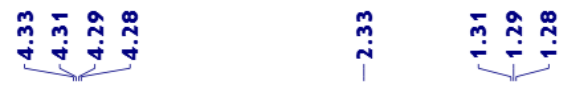
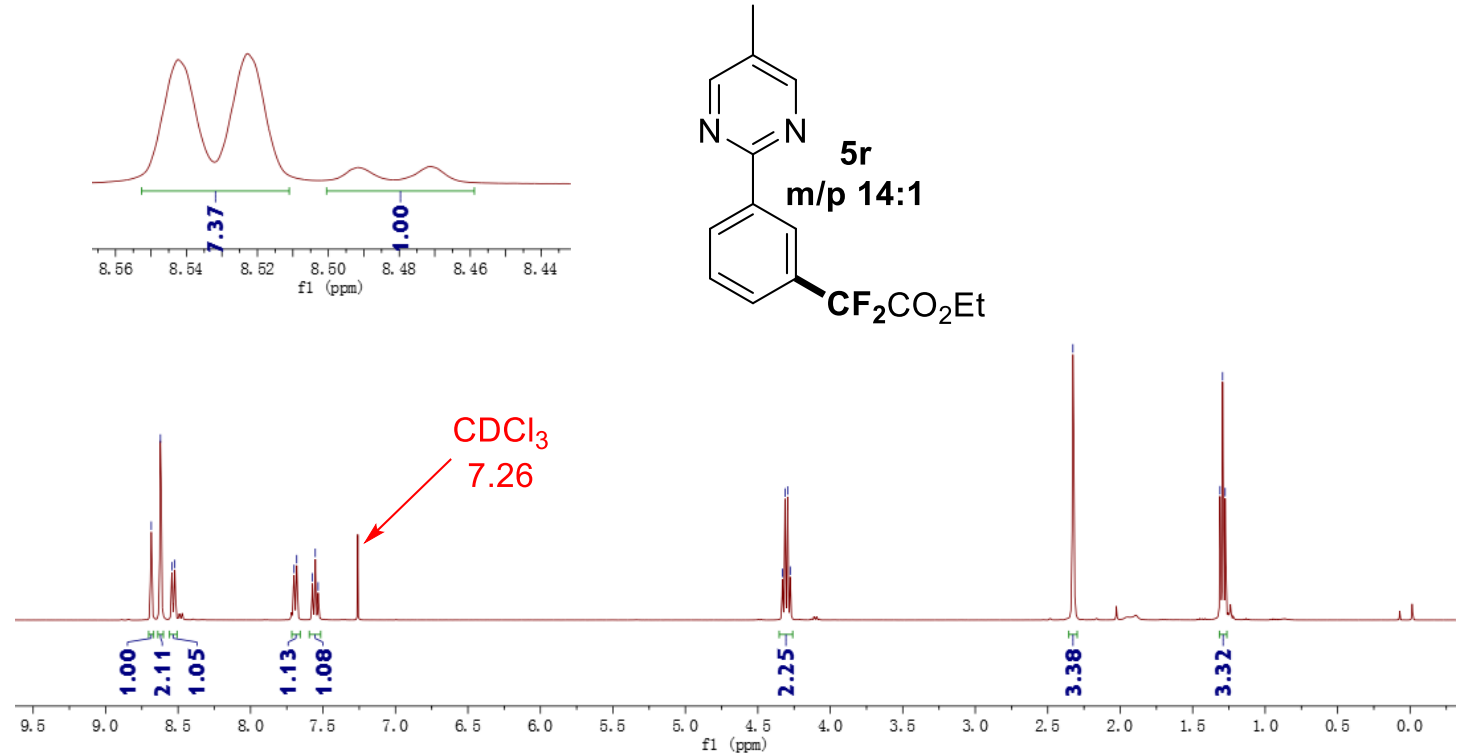
${ }^{19}$ F NMR (376 MHz, $\left.\mathrm{CDCl}_{3}\right)$ of $5 r$

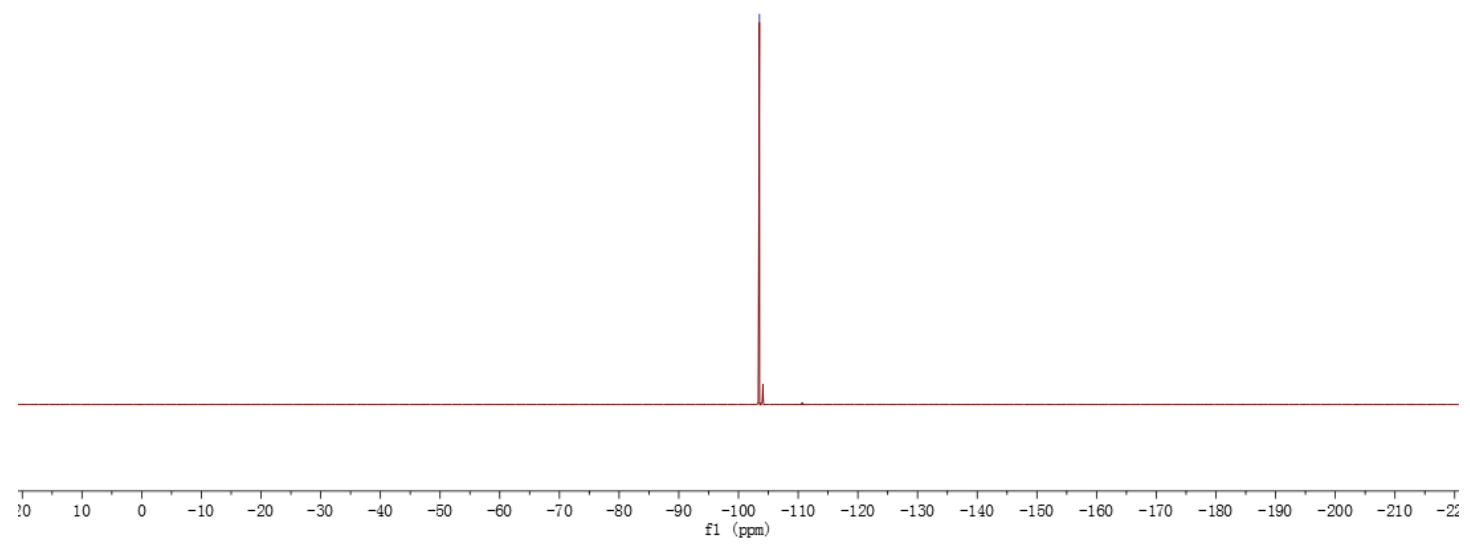

${ }^{13} \mathrm{C}$ NMR (100 MHz, $\left.\mathrm{CDCl}_{3}\right)$ of $5 \mathrm{r}$
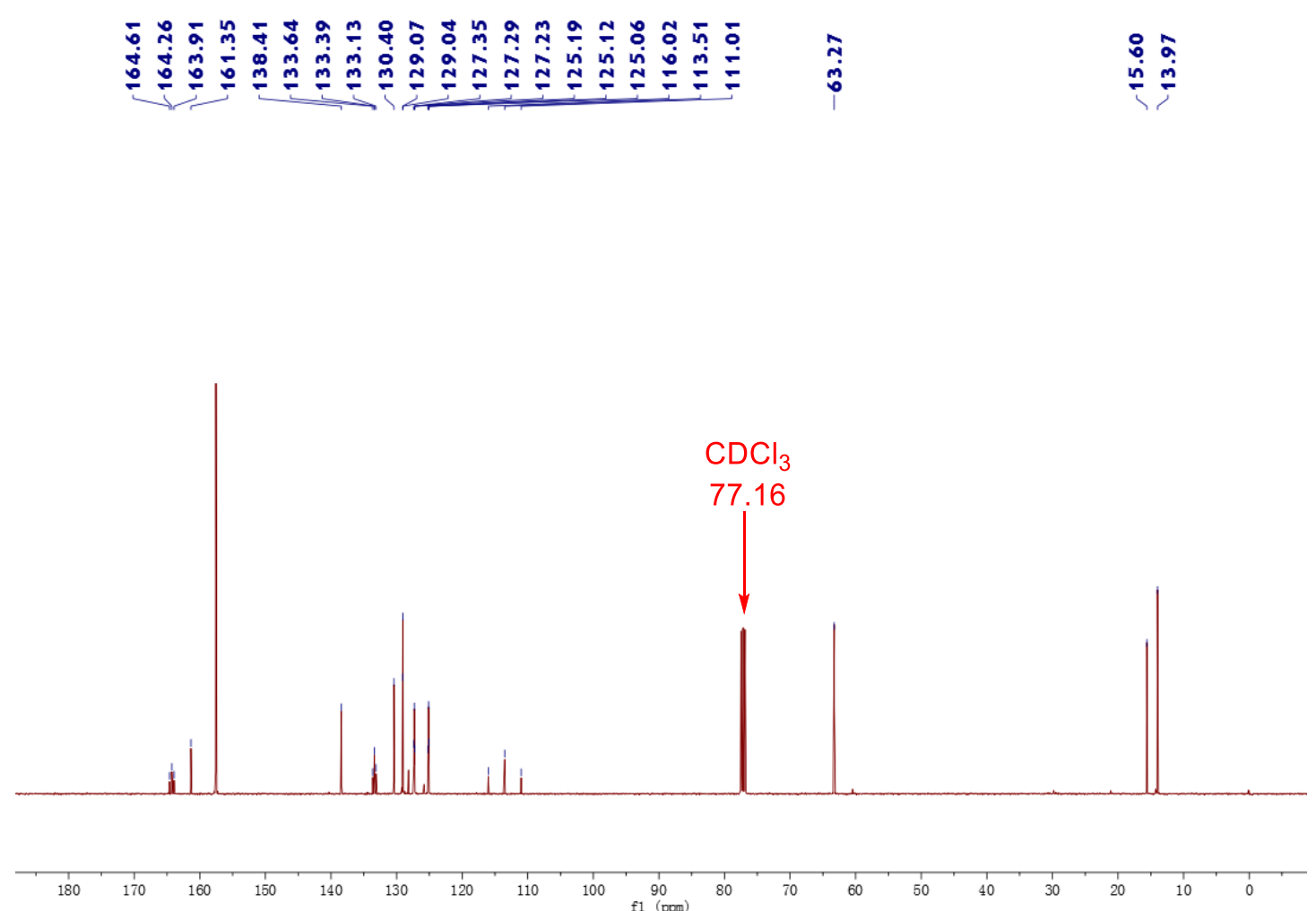
${ }^{1} \mathrm{H}$ NMR (400 MHz, $\left.\mathrm{CDCl}_{3}\right)$ of 11

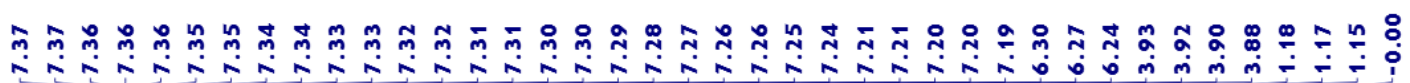<smiles>CCOC(=O)[C-](C=C(c1ccccc1)c1ccccc1)c1ccccc1</smiles>

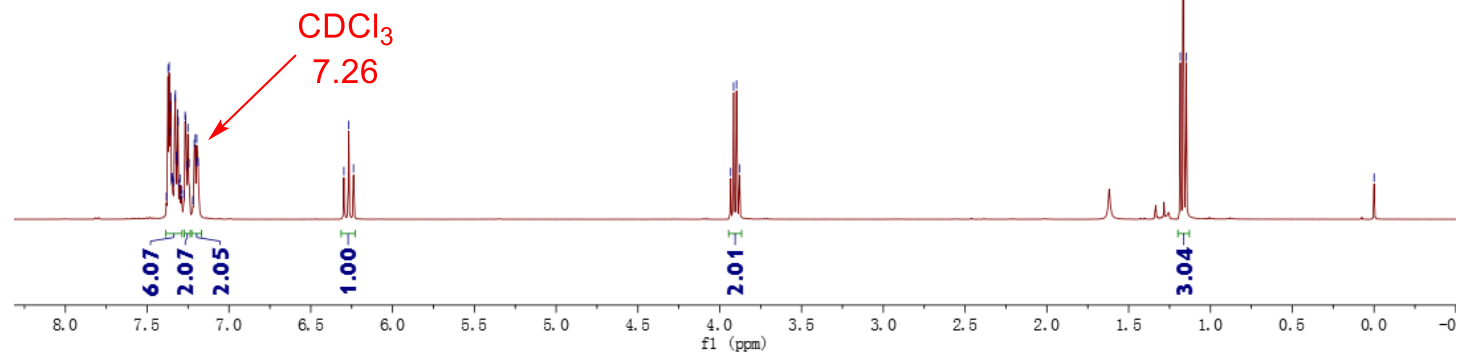

${ }^{1} \mathrm{H}$ NMR (400 MHz, $\left.\mathrm{CDCl}_{3}\right)$ of $5 \mathrm{a}-\left[\mathrm{D}_{4}\right]$

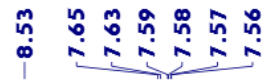<smiles>[2H]c1c([2H])c(C([2H])([2H])[18O]CC)c([2H])c([2H])c1-c1ccc(C)cn1</smiles>

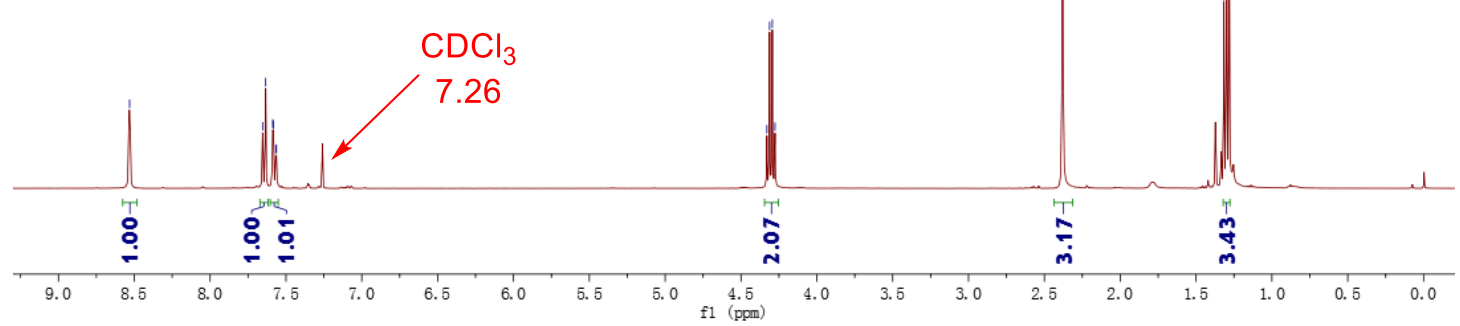

